Florida International University FIU Digital Commons

4-16-2018

\title{
Conformational Dynamics of Biomolecules by Trapped Ion Mobility Spectrometry Dynamics
}

Juan Camilo Molano-Arévalo

Florida International University, jmola004@fiu.edu

DOI: $10.25148 /$ etd.FIDC006557

Follow this and additional works at: https://digitalcommons.fiu.edu/etd

Part of the Analytical Chemistry Commons, and the Structural Biology Commons

\section{Recommended Citation}

Molano-Arévalo, Juan Camilo, "Conformational Dynamics of Biomolecules by Trapped Ion Mobility Spectrometry Dynamics" (2018). FIU Electronic Theses and Dissertations. 3647.

https://digitalcommons.fiu.edu/etd/3647 


\section{FLORIDA INTERNATIONAL UNIVERSITY}

Miami, Florida

\section{CONFORMATIONAL DYNAMICS OF BIOMOLECULES BY TRAPPED ION MOBILITY SPECTROMETRY AND MOLECULAR DYNAMICS}

A dissertation submitted in partial fulfillment of

the requirements for the degree of

DOCTOR OF PHILOSOPHY

in

\section{CHEMISTRY}

by

Juan Camilo Molano-Arévalo

2018 
To: Dean Michael R. Heithaus

College of Arts, Sciences and Education

This dissertation, written by Juan Camilo Molano-Arévalo, and entitled Conformational Dynamics of Biomolecules by Trapped Ion Mobility Spectrometry and Molecular Dynamics, having been approved in respect to style and intellectual content, is referred to you for judgment.

We have read this dissertation and recommend that it be approved.

$\begin{array}{r}\hline \text { Jeff Joens } \\ \hline \text { Fenfei Leng } \\ \hline \text { Jessica Liberles } \\ \hline \text { Alexander Mebel } \\ \hline\end{array}$

Date of Defense: April 16, 2018

The dissertation of Juan Camilo Molano-Arévalo is approved.

Dean Michael R. Heithaus College of Arts, Sciences and Education

Andrés G. Gil

Vice President for Research and Economic Development and Dean of the University Graduate School

Florida International University, 2018 
(C) Copyright 2018 by Juan Camilo Molano-Arévalo

All rights reserved. 


\section{DEDICATION}

To my parents. 
ABSTRACT OF THE DISSERTATION

\title{
CONFORMATIONAL DYNAMICS OF BIOMOLECULES BY TRAPPED ION MOBILITY SPECTROMETRY AND MOLECULAR DYNAMICS
}

by

Juan Camilo Molano Arévalo

Florida International University, 2018

\author{
Miami, Florida
}

\section{Professor Francisco Fernández-Lima, Major Professor}

One of the main goals in structural biology is to understand the folding mechanisms and three-dimensional structure of biomolecules. Many biomolecular systems adopt multiple structures as a function of their microenvironment, which makes them difficult to be characterized by traditional structural biology tools (e.g., NMR, X-ray crystallography). As an alternative, complementary tools that can capture and sample multiple conformations needed to be developed. In the present work, we pioneered the application of a new variant of ion mobility spectrometry, trapped ion mobility spectrometry (TIMS), which provides high mobility resolving power and the possibility to study kinetically trapped intermediates as a function of the starting solution (e.g., $\mathrm{pH}$ and organic content) and gas-phase conditions (e.g., collisional activation, molecular dopants, hydrogen/deuterium back- 
exchange). When coupled to mass spectrometry (TIMS-MS), action spectroscopy (IRMPD), molecular dynamics and biochemical approaches (e.g., fluorescence lifetime spectroscopy), a comprehensive description of the biomolecules dynamics and tridimensional structural can be obtained. These new set of tools were applied for the first time to the study of Flavin Adenine Dinucleotide (FAD), Nicotineamide Adenine Dinucleotide (NAD), globular protein cytochrome c (cyt c), the $3{ }_{1}$ knot YibK protein, 52 knot ubiquitin $\mathrm{C}$ terminal hydrolase $(\mathrm{UCH})$ protein, and the $6_{1}$ knot halo acid dehydrogenase (DehI) protein. 
CHAPTER 2. FLAVIN ADENINE DINUCLEOTIDE STRUCTURAL MOTIFS: FROM SOLUTION TO GAS-PHASE

CHAPTER 3. INSIGHTS FROM ION MOBILITY - MASS SPECTROMETRY, INFRARED SPECTROSCOPY, AND MOLECULAR DYNAMICS ON NICOTINAMIDE ADENINE DINUCLEOTIDE STRUCTURAL DYNAMICS: $\mathrm{NAD}^{+}$VS NADH.

CHAPTER 4. CHARACTERIZATION OF INTRAMOLECULAR INTERACTIONS OF CYTOCHROME C USING HYDROGEN DEUTERIUM EXCHANGE TRAPPED ION MOBILITY - MASS SPECTROMETRY AND MOLECULAR DYNAMICS

CHAPTER 5. CHARACTERIZATION OF THE $3{ }_{1}$ KNOT PROTEIN YIBK USING ENZYMATIC DIGESTION. MOLECULAR DYNAMICS AND TRAPPED ION MOBILITY SPECTROMETRY - MASS SPECTROMETRY

CHAPTER 6. INSIGHTS FROM TRAPPED ION MOBILITY SPECTROMETRY MASS SPECTROMETRY ON THE STRUCTURAL INTEGRITY OF THE KNOT PROTEIN UBIQUITIN C-TERMINAL HYDROLASE.

CHAPTER 7. ELUCIDATION OF THE STRUCTURAL INTEGRITY AND STABILITY OF THE KNOT PROTEIN HALO ACID DEHALOGENASE USING TRAPPED ION MOBILITY SPECTROMETRY - MASS SPECTROMETRY

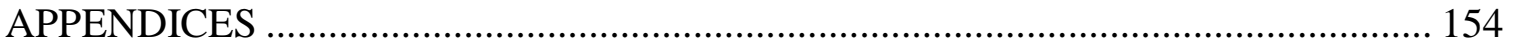

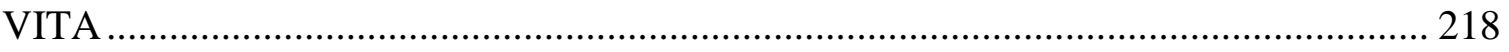




\section{LIST OF FIGURES}

Figure 2.1 Structure and typical MS spectra for the deprotonated $\left(\mathrm{M}=\mathrm{C}_{27} \mathrm{H}_{31} \mathrm{~N}_{9} \mathrm{O}_{15} \mathrm{P}_{2}\right)$ and protonated $\left(\mathrm{M}=\mathrm{C}_{27} \mathrm{H}_{33} \mathrm{~N}_{9} \mathrm{O}_{15} \mathrm{P}_{2}\right)$ FAD forms. Monoisotopic peaks of the $[\mathrm{M}+\mathrm{H}]^{+}$ and $[\mathrm{M}+\mathrm{Na}]^{+}$molecular ions used in the mobility analysis are highlighted with a dashed rectangle.

Figure 2.2 Typical TIMS spectra as a function of the organic content for the $[\mathrm{M}+\mathrm{H}]^{+}$ and $[\mathrm{M}+\mathrm{Na}]^{+}$molecular ions of the deprotonated $\left(\mathrm{M}=\mathrm{C}_{27} \mathrm{H}_{31} \mathrm{~N}_{9} \mathrm{O}_{15} \mathrm{P}_{2}\right)$ and protonated $\left(\mathrm{M}=\mathrm{C}_{27} \mathrm{H}_{33} \mathrm{~N}_{9} \mathrm{O}_{15} \mathrm{P}_{2}\right)$ FAD forms.

Figure 2.3 Frequency domain phase delay (solid squares) and modulation ratio (open circles) of $10 \mu \mathrm{M}$ FAD in mixture of a) ethanol: water, b) methanol: water and c) 10 $\mu \mathrm{M}$ FAD in mixture of ammonium acetate buffer: water as a function of the volume ratio of 0:100 (black line), 30:70 (red line), (50:50) green line and 70:30 (blue line). Solid lines represent the best to the data using a triple exponential decay.

Figure 2.4 Candidate structures for the IMS bands observed for the $[\mathrm{M}+\mathrm{H}]^{+}$and $[\mathrm{M}+\mathrm{Na}]^{+}$molecular ions of the deprotonated $\left(\mathrm{M}=\mathrm{C}_{27} \mathrm{H}_{31} \mathrm{~N}_{9} \mathrm{O}_{15} \mathrm{P}_{2}\right)$ and protonated $\left(\mathrm{M}=\mathrm{C}_{27} \mathrm{H}_{33} \mathrm{~N}_{9} \mathrm{O}_{15} \mathrm{P}_{2}\right)$ FAD forms.

Figure 2.5 Typical IMS spectra of the $[\mathrm{M}+\mathrm{Na}]^{+}$ions of the protonated FAD form $\left(\mathrm{M}=\mathrm{C}_{27} \mathrm{H}_{33} \mathrm{~N}_{9} \mathrm{O}_{15} \mathrm{P}_{2}\right)$ as a function of the bath gas conditions. Notice the variation of the relative abundances of IMS bands as a function of the bath gas composition using ethanol, methanol and acetonitrile as additives in the TIMS mobility cell.

Figure 3.1 Structures of the oxidized $\left(\mathrm{M}=\mathrm{C}_{21} \mathrm{H}_{27} \mathrm{~N}_{7} \mathrm{O}_{14} \mathrm{P}_{2}\right)$ and reduced $(\mathrm{M}=$ $\left.\mathrm{C}_{21} \mathrm{H}_{29} \mathrm{~N}_{7} \mathrm{O}_{14} \mathrm{P}_{2}\right)$ NAD forms. Typical MS spectra of protonated and sodiated molecular ions of both NAD species are shown.

Figure 3.2 Ion mobility profiles of NAD $\left(t_{\text {trap }}=500 \mathrm{~ms}\right)$ as a function of the organic content in the starting solution (blue panel) and the collision induced activation energy (red panel). Labels A-D are assigned to the IMS bands of the NADH $[\mathrm{M}+\mathrm{H}]^{+}$species; E-F to NADH $[\mathrm{M}+\mathrm{Na}]^{+}$species; $\mathrm{G}-\mathrm{H}$ to $\mathrm{NAD}^{+}[\mathrm{M}+\mathrm{H}]^{+}$species; and I-J to NAD ${ }^{+}$ $[\mathrm{M}+\mathrm{Na}]^{+}$species. 
Figure 3.3 Typical IMS and interconversion plots as a function of the trapping time and starting solvent conditions $\left(70: 30 \mathrm{H}_{2} \mathrm{O}: \mathrm{MeOH}\right.$ and $\left.\mathrm{H}_{2} \mathrm{O}: \mathrm{EtOH}\right)$ for $\mathrm{NADH}$ $[\mathrm{M}+\mathrm{H}]^{+}$and $[\mathrm{M}+\mathrm{Na}]^{+}$species.

Figure 3.4 Emission spectra of NAD as a function of the organic content. In the inset, changes in the pre-exponential factor $(\alpha)$ is shown as a function organic content for the fast $\left(\tau_{0}\right)$ and slow $\left(\tau_{1}\right)$ components.

Figure 3.5 Experimental and theoretical IRMPD spectra for the protonated NADH (blue) and $\mathrm{NAD}^{+}$(red) forms. The three lowest energy candidate structures per mobility band and corresponding IR spectra are shown. The boxes represent simplified calculated IR spectra to facilitate the visualization (complete calculated IR spectra are provided in Figure S8).

Figure 4.1 Left panel: typical mass spectra of cyt $\mathrm{c}$ as a function of the starting solvent conditions. Right panel: overall CCS profiles (black lines) obtained by summation of the intensity-normalized IMS resolved data (color lines). The results obtained in the positive and negative ion mode are represented on the top and bottom part, respectively.

Figure 4.2 Left panel: IMS spectra of the +6 to +11 charges states of cyt $\mathrm{c}$ as a function of the organic content (e.g., \% methanol). Right panel: IMS spectra of the +6 to +11 charge states of cyt $\mathrm{c}$ as a function of the activation energy (e.g., deflector voltage)...... 84

Figure 4.3 Bottom: IMS profiles obtained with CIA (blue line), and without CIA (green line). Top: High resolution IMS profiles for the +12 to +21 charge states of cyt

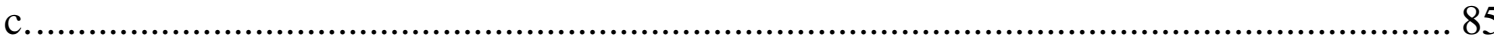

Figure 4.4 HDX back exchange as a function of the incubation time (e.g., 3 and $48 \mathrm{~h}$ ) and the time after desolvation (e.g., 0, 100, and $500 \mathrm{~ms}$ ), in terms of charge state and CCS in the positive ion mode. Clustering of the number of kinetically trapped intermediates and the initial levels of exchange allows the identification of four groups with different levels of exchange protection (color rectangles and data points).

Figure 4.5 Candidate structures proposed for the kinetic intermediates IMS bands of cyt c. Conformations $\mathrm{G}$ and $\mathrm{H}$ for the +7 charge state (dark red background); and $\mathrm{C}$, $\mathrm{D}$, and $\mathrm{E}$ (dark green background), for the +8 , and +9 charge states, were obtained after CIA.

Figure 5.1 Typical mass spectra of YibK in the positive mode as a function of the starting solvent conditions. Overall $\mathrm{CCS}_{\mathrm{N} 2}$ profiles (black lines) obtained by summation of the intensity-normalized IMS resolved data (color lines). Green background, native-like states; blue background: transition states; red background: unfolded states. 
Figure 5.2 IMS profiles of the +7 to +22 charge states of YibK as a function of the organic content (e.g., \% MeOH) in the starting solution. Molecular representations and theoretical $\mathrm{CCS}_{\mathrm{N} 2}$ of the native/knotted (blue triangles) and denatured/unfolded (red triangles) of YibK are shown. IMS profiles of the +8 to +22 charge states of YibK as a function of the CIA regime (e.g., on/off) for a solution with $10 \% \mathrm{MeOH}$.

Figure 5.3 Overall $\mathrm{CCS}_{\mathrm{N} 2}$ profiles (black lines) obtained by summation of the intensity-normalized IMS resolved data (color lines) obtained as a function of the starting solution temperature.

Figure 5.4 Digestion of YibK with CPDY. A: $\mathrm{CCS}_{\mathrm{N} 2}$ distribution of the observed fragments as a function of the mass of each fragment. Orange circles: undigested protein; Green triangles: fragments with residues removed exclusively from the $\mathrm{C}$ terminal; Red triangles: fragments with residues removed exclusively from the $\mathrm{N}$ terminal; Blue rhomboids: fragments with residues removed from the both termini; Black circles: fragments where a structural collapse is observed; Black stars and grey line: fragments where the knot is preserved after the digestion; Blue line: distribution of reported $\mathrm{CCS}_{\mathrm{N} 2}$ for globular proteins. B: Sequence coverage by the digestion. C: IMS profiles of the fragments that preserve the knot.

Figure 6.1 Typical mass spectra of UCH in the positive mode as a function of the starting solvent conditions. Overall $\mathrm{CCS}_{\mathrm{N} 2}$ profiles of $\mathrm{UCH}$ (black lines) obtained by summation of the intensity-normalized IMS resolved data (color lines).

Figure 6.2 IMS profiles of the +8 to +26 charge states of $\mathrm{UCH}$ as a function of the organic content (e.g., $\% \mathrm{MeOH}$ ) in the starting solution. IMS profiles of the +8 to +26 charge states of UCH as a function of the CIA regime (e.g., on/off) for a solution with $10 \% \mathrm{MeOH}$. The blue profiles were obtained when the starting solution did not contain any amount of methanol and the CIA energy was off (e.g., $\mathrm{V}_{\text {cap }}=50 \mathrm{~V}, \mathrm{~V}_{\text {def }}=60 \mathrm{~V}$, and $\mathrm{V}_{\text {fun }}=0 \mathrm{~V}$ ). The red profiles were obtained when $50 \%$ of the starting solution was methanol and the CIA was off. The yellow profiles were obtained when $10 \%$ of $\mathrm{MeOH}$ was added to the starting solution and the CIA energy was off. Finally, the green profiles were obtained when the starting solution had $10 \% \mathrm{MeOH}$ and the CIA energy was on.

Figure 6.3 Overall $\mathrm{CCS}_{\mathrm{N} 2}$ profiles for $\mathrm{UCH}$ (black lines) obtained by summation of the intensity-normalized IMS resolved data (color lines) obtained as a function of the temperature of the starting solution with $0 \% \mathrm{MeOH}$, and $10 \% \mathrm{MeOH}$..

Figure 6.4 Digestion of $\mathrm{UCH}$ with CPDY. A: $\mathrm{CCS}_{\mathrm{N} 2}$ distribution of the observed fragments as a function of the mass of each fragment. Orange circles: undigested protein; Green triangles: fragments with residues removed exclusively from the $\mathrm{C}$ terminal; Red triangles: fragments with residues removed exclusively from the Nterminal; Blue rhomboids: fragments with residues removed from the both termini; Black circles: fragments where a structural collapse is observed; Black stars and grey line: fragments where the knot is preserved after the digestion; Blue line: distribution 
of reported $\mathrm{CCS}_{\mathrm{N} 2}$ for globular proteins. B: Sequence coverage by the digestion. C: IMS profiles of the fragments that preserve the knot.

Figure 7.1 Typical mass spectra of DehI in the positive mode as a function of the starting solvent conditions. Overall $\mathrm{CCS}_{\mathrm{N} 2}$ profiles of DehI (black lines) obtained by summation of the intensity-normalized IMS resolved data (color lines).

Figure 7.2 IMS profiles of the +8 to +31 charge states of DehI as a function of the organic content (e.g., $\% \mathrm{MeOH}$ ) in the starting solution. IMS profiles of the +8 to +31 charge states of DehI as a function of the CIA regime (e.g., on/off) for a solution with $10 \% \mathrm{MeOH}$. The blue profiles were obtained when the starting solution did not contain any amount of methanol and the CIA energy was off (e.g., $\mathrm{V}_{\text {cap }}=50 \mathrm{~V}, \mathrm{~V}_{\text {def }}=60 \mathrm{~V}$, and $\mathrm{V}_{\text {fun }}=0 \mathrm{~V}$ ). The red profiles were obtained when $50 \%$ of the starting solution was methanol and the CIA was off. The yellow profiles were obtained when $10 \%$ of $\mathrm{MeOH}$ was added to the starting solution and the CIA energy was off. Finally, the green profiles were obtained when the starting solution had $10 \% \mathrm{MeOH}$ and the CIA energy was on.

Figure 7.3 Overall $\mathrm{CCS}_{\mathrm{N} 2}$ profiles for DehI (black lines) obtained by summation of the intensity-normalized IMS resolved data (color lines) obtained as a function of the temperature of the starting solution.

Figure 7.4 Digestion of DehI with CPDY. A: $\mathrm{CCS}_{\mathrm{N} 2}$ distribution of the observed fragments as a function of the mass of each fragment. Orange circles: undigested protein; Green triangles: fragments with residues removed exclusively from the $\mathrm{C}$ terminal; Red triangles: fragments with residues removed exclusively from the $\mathrm{N}$ terminal; Blue rhomboids: fragments with residues removed from the both termini; Black circles: fragments where a structural collapse is observed; Black stars and grey line: fragments where the knot is preserved after the digestion. B: Sequence coverage by the digestion. C: IMS profiles of the fragments that preserve the knot. 
CHAPTER 1.

INTRODUCTION 
Traditionally, the study of biomolecular conformations has focused on the condensed phase by well established methodologies, such as nuclear magnetic resonance, crystallography, cryo-electron microscopy, circular dichroism, among others. ${ }^{1}$ However, these techniques pose unavoidable challenges when the research interest deviates from the study of the native conformation: the structure of disordered proteins cannot be resolved in detail by the aforementioned techniques as they are problematic to crystallize; the conformational dynamics of proteins can only be analyzed by a handful of practices and only broad conclusions can be derived from such studies; and many of the kinetic intermediates that are involved in the folding of proteins cannot be isolated or identified, complicating the proposal of folding mechanisms. ${ }^{2,3}$

In the gas-phase, a world of analytical possibilities opens for the thorough study of the conformational dynamics and folding pathways of proteins and biomolecules. By using mass spectrometry (MS) based techniques, we can isolate and identify molecules (i.e., molecular identification), we can study the intramolecular interactions that define the conformation of molecules (i.e., infrared spectroscopy, IR), we can analyze and fingerprint the accessible surface area of proteins and peptides (i.e., hydrogen/deuterium exchange, HDX), and we can isolate and measure the size of the trapped kinetic intermediates that are part the folding pathway of biomolecules (i.e., ion mobility spectrometry, IMS). ${ }^{4-6}$ All these procedures allow the unambiguous selection and isolation of the target analytes, their study in the absence of solvents, or in the presence of selected of collisional partners.

The main goal of the projects presented here was to propose and implement analytical methodologies for the analysis of the conformational dynamics of biomolecules by using 
trapped ion mobility spectrometry (TIMS). Unlike other IMS variants, TIMS permits the interrogation and manipulation of mobility separated ion populations in the gas-phase with high resolving power. In TIMS, the mobility separation is based on holding the ion packages stationary using an electric field that compensates the exerted drift force against a moving bath gas. This allows the molecular separation based on the ion mobility of each package, which in turn is dependent on the size and shape of each trapped intermediate. The ions are also radially confined by a quadrupolar field, significantly increasing the transmission and sensitivity, compared to other IMS instruments. Since the separation is related to the number of ion-neutral collisions, using first principles we can measure the ion-neutral collision cross section (CCS) of each mobility-resolved trapped intermediate. ${ }^{7-}$

10 These measurements, combined with molecular dynamics, permits the structural assignment of the candidate structures that populate the conformational landscape of biomolecules. $^{7,8,10-12}$ In our group, we have successfully showed the application of TIMS based techniques on the study of inorganic ${ }^{13-17}$ and organic molecules, ${ }^{18,19}$ oligonucleotides ${ }^{20}$ peptides, ${ }^{21-23}$ and proteins. ${ }^{24-27}$ The fast analyses and high mobility separations routinely achieved, makes TIMS an attractive alternative, and perhaps a staple, in the growing fields of structural biology, proteomics, metabolomics, and lipidomics.

During the development of this dissertation, several complimentary techniques using TIMS-MS were proposed and their results were published for the first time, such as the thermalization kinetics of intermediates in the millisecond timescale, the gas-phase solvation effects of dopants in the TIMS cell, the implementation of collision induced activation (CIA) for the interrogation of other than solution accessible intermediates, and 
the measurement of hydrogen/deuterium back-exchange rates of mobility selected trapped intermediates. The following chapters provide a better understanding on the conformational dynamics of biomolecules studied with TIMS, and the wide variety of complimentary approaches employed.

Chapter 2, "Flavin adenine dinucleotide structural motifs: from solution to gas-phase", published in Analytical Chemistry in 2014, ${ }^{19}$ describes the conformational changes of Flavin Adenine Dinucleotide (FAD) in solution and in the gas-phase by measuring the fluorescence time decay and ion-neutral collision cross sections as a function of the solvent conditions and gas-phase collisional partner. Our results showed that FAD can exist in four conformations in solution, where the abundance of the extended conformations increases with the organic content. TIMS-MS experiments showed that FAD can exist in the gasphase as deprotonated and protonated forms and that 12 conformations can be observed as a function of the starting solution for the $[\mathrm{M}+\mathrm{H}]^{+}$and $[\mathrm{M}+\mathrm{Na}]^{+}$molecular ions. Additionally, changes in the relative abundances of the gas-phase structures were observed from a "stack" to a "close" conformation when organic molecules were introduced in the TIMS cell as collision partners.

In chapter 3, "Insights from Ion Mobility - Mass Spectrometry, Infrared Spectroscopy, and Molecular Dynamics on Nicotinamide Adenine Dinucleotide Structural Dynamics: NAD vs NADH", published in Physical Chemistry Chemical Physics in $2018,{ }^{28}$ we propose a new analytical workflow for study $\mathrm{NAD}^{+}$and $\mathrm{NADH}$ dynamics as a function of the organic content in solution using fluorescence lifetime spectroscopy and in the gas-phase using nESI-TIMS-MS and infrared multiple photon dissociation (IRMPD). Our results suggest 
that in solution, NAD can change from a "close" to "open" conformation with the increase of the organic content. NAD gas-phase studies using nESI-TIMS-MS displayed two ion mobility bands for $\mathrm{NAD}^{+}$protonated and sodiated species, while four and two ion mobility bands were observed for NADH protonated and sodiated species, respectively. Changes in the mobility profiles were observed for NADH as a function of the starting solution condition and the time after desolvation, while $\mathrm{NAD}^{+}$profiles showed no dependence. The IRMPD spectroscopy of $\mathrm{NAD}^{+}$and NADH protonated species in the 800-1800 and 3200$3700 \mathrm{~cm}^{-1}$ spectral regions showed common and signature bands between the NAD forms.

In chapter 4, "Characterization of Intramolecular Interactions of Cytochrome c Using Hydrogen-Deuterium Exchange-Trapped Ion Mobility Spectrometry-Mass Spectrometry and Molecular Dynamics", published in Analytical Chemistry in 2017, ${ }^{27}$ we showed the advantages of TIMS-MS with respect to previously IMS-MS studies for the case of a model globular protein Cytochrome c (cyt c). The structural interrogation of kinetically trapped intermediates of cyt $\mathrm{c}$ was performed by correlating the CCS and charge state with the starting solution conditions and time after desolvation using CIA, time-resolved HDX and TIMS-MS. The high ion mobility resolving power of the TIMS analyzer allowed the identification of new ion mobility bands, yielding a total of 63 mobility bands over the +6 to +21 charge states and 20 mobility bands over the -5 to -10 charge states. Mobility selected HDX rates showed that for the same charge state, conformers with larger CCS present faster HDX rates in both positive and negative ion mode, suggesting that the charge sites and neighboring exchange sites on the accessible surface area define the exchange rate regardless of the charge state. Complementary molecular dynamic simulations 
permitted the generation of candidate structures and a mechanistic model of the folding transitions from native $(\mathrm{N})$ to molten globule $(\mathrm{MG})$ to kinetic intermediates $(\mathrm{U})$ pathways. Our results suggest that cyt $\mathrm{c}$ major structural unfolding is associated with the distancing of the $\mathrm{N}$ - and $\mathrm{C}$-terminal helices and subsequent solvent exposure of the hydrophobic, heme-containing cavity.

Finally, in chapter 5, "Structural Characterization of the Knot Protein YibK using Trapped Ion Mobility Spectrometry - Mass Spectrometry and Enzymatic Digestion”; chapter 6, "Insights from Trapped Ion Mobility Spectrometry - Mass Spectrometry on the Conformation of the Knot Protein Ubiquitin C terminal Hydrolase”; and chapter 7 "Elucidation of the Structural Integrity and Stability of the knot protein halo acid dehydrogenase using Trapped Ion Mobility Spectrometry - Mass Spectrometry", we discuss the conformation of the $3_{1}, 5_{2}$, and 6 knots found in the proteins YibK, $\mathrm{UCH}$, and DehI using TIMS-MS based tools, respectively. The nascent area of research on knotted proteins requires new experimental approaches to provide conclusive answers about the roles of knots in proteins, and TIMS-MS provided sufficient information for the elucidation of the folding dynamics of these particular conformations. Using a combination of TIMSMS based techniques, we studied the integrity, and stability of the aforementioned knots as a function of the solution conditions, the activation energy, and the cooperative effect of temperature and the starting solution composition on the conformational space. Additionally, we developed a novel methodology using TIMS-MS, enzymatic digestion and molecular dynamics to better characterize the knot core and knotted proteins. These findings are under review in the Journal of the American Society of Mass Spectrometry. 


\section{LIST OF REFERENCES}

(1) Banaszak, L. Foundations of Structural Biology, 2000.

(2) Dyson, H. J.; Wright, P. E. Nat Rev Mol Cell Biol 2005, 6, 197-208.

(3) Dunker, A. K.; Lawson, J. D.; Brown, C. J.; Williams, R. M.; Romero, P.; Oh, J. S.; Oldfield, C. J.; Campen, A. M.; Ratliff, C. M.; Hipps, K. W.; Ausio, J.; Nissen, M. S.; Reeves, R.; Kang, C.; Kissinger, C. R.; Bailey, R. W.; Griswold, M. D.; Chiu, W.; Garner, E. C.; Obradovic, Z. Journal of molecular graphics \& modelling 2001, 19, 26-59.

(4) Wysocki, V. H.; Resing, K. A.; Zhang, Q.; Cheng, G. Methods (San Diego, Calif.) 2005, 35, 211-222.

(5) Engen, J. R.; Smith, D. L. Anal Chem 2001, 73, 256A-265A.

(6) Little, D. P.; Speir, J. P.; Senko, M. W.; O'Connor, P. B.; McLafferty, F. W. Anal Chem 1994, 66, 2809-2815.

(7) Fernandez-Lima, F.; Kaplan, D. A.; Suetering, J.; Park, M. A. International journal for ion mobility spectrometry : official publication of the International Society for Ion Mobility Spectrometry 2011, 14, 93-98.

(8) Fernandez-Lima, F. A.; Kaplan, D. A.; Park, M. A. The Review of scientific instruments 2011, 82, 126106.

(9) McDaniel, E. W.; Mason, E. A. Mobility and diffusion of ions in gases; John Wiley and Sons, Inc., New York: New York, 1973, p 381.

(10) Hernandez, D. R.; Debord, J. D.; Ridgeway, M. E.; Kaplan, D. A.; Park, M. A.; Fernandez-Lima, F. Analyst 2014, 139, 1913-1921.

(11) Fernandez-Lima, F. A.; Wei, H.; Gao, Y. Q.; Russell, D. H. J Phys Chem A 2009, 113, 8221-8234.

(12) Fernandez-Lima, F.; Blase, R. C.; Russell, D. H. International Journal of Mass Spectrometry 2011, 298, 111-118.

(13) Castellanos, A.; Benigni, P.; Hernandez, D. R.; DeBord, J. D.; Ridgeway, M. E.; Park, M. A.; Fernandez-Lima, F. Anal Methods 2014, 6, 9328-9332.

(14) Benigni, P.; Thompson, C. J.; Ridgeway, M. E.; Park, M. A.; Fernandez-Lima, F. Anal Chem 2015, 87, 4321-4325.

(15) McKenzie-Coe, A.; DeBord, J. D.; Ridgeway, M.; Park, M.; Eiceman, G.; FernandezLima, F. Analyst 2015, 140, 5692-5699. 
(16) Adams, K. J.; Montero, D.; Aga, D.; Fernandez-Lima, F. International journal for ion mobility spectrometry : official publication of the International Society for Ion Mobility Spectrometry 2016, 19, 69-76.

(17) Benigni, P.; Bravo, C.; Quirke, J. M. E.; DeBord, J. D.; Mebel, A. M.; FernandezLima, F. Energ Fuel 2016, 30, 10341-10347.

(18) Schenk, E. R.; Mendez, V.; Landrum, J. T.; Ridgeway, M. E.; Park, M. A.; FernandezLima, F. Anal Chem 2014, 86, 2019-2024.

(19) Molano-Arevalo, J. C.; Hernandez, D. R.; Gonzalez, W. G.; Miksovska, J.; Ridgeway, M. E.; Park, M. A.; Fernandez-Lima, F. Anal Chem 2014, 86, 10223-10230.

(20) Garabedian, A.; Butcher, D.; Lippens, J. L.; Miksovska, J.; Chapagain, P. P.; Fabris, D.; Ridgeway, M. E.; Park, M. A.; Fernandez-Lima, F. Phys Chem Chem Phys 2016, 18, 26691-26702.

(21) Schenk, E. R.; Ridgeway, M. E.; Park, M. A.; Leng, F.; Fernandez-Lima, F. Anal Chem 2014, 86, 1210-1214.

(22) Frost, L.; Baez, M. A. M.; Harrilal, C.; Garabedian, A.; Fernandez-Lima, F.; Leng, F. In PLoS One, 2015.

(23) Garabedian, A.; Benigni, P.; Ramirez, C. E.; Baker, E. S.; Liu, T.; Smith, R. D.; Fernandez-Lima, F. J Am Soc Mass Spectrom 2017.

(24) Schenk, E. R.; Almeida, R.; Miksovska, J.; Ridgeway, M. E.; Park, M. A.; FernandezLima, F. J Am Soc Mass Spectrom 2015, 26, 555-563.

(25) Gonzalez, W. G.; Ramos, V.; Diaz, M.; Garabedian, A.; Molano-Arevalo, J. C.; Fernandez-Lima, F.; Miksovska, J. Biochemistry 2016, 55, 1873-1886.

(26) Benigni, P.; Marin, R.; Molano-Arevalo, J. C.; Garabedian, A.; Wolff, J. J.; Ridgeway, M. E.; Park, M. A.; Fernandez-Lima, F. International journal for ion mobility spectrometry : official publication of the International Society for Ion Mobility Spectrometry 2016, 19, 95-104.

(27) Molano-Arevalo, J. C.; Jeanne Dit Fouque, K.; Pham, K.; Miksovska, J.; Ridgeway, M. E.; Park, M. A.; Fernandez-Lima, F. Anal Chem 2017, 89, 8757-8765.

(28) Molano-Arevalo, J. C.; Gonzalez, W.; Jeanne Dit Fouque, K.; Miksovska, J.; Maitre, P.; Fernandez-Lima, F. Phys Chem Chem Phys 2018, 20, 7043-7052. 


\section{CHAPTER 2.}

\section{FLAVIN ADENINE DINUCLEOTIDE STRUCTURAL MOTIFS: FROM SOLUTION TO GAS-PHASE}

This chapter was published in Analytical Chemistry and adapted with permission from all authors.

J. C. Molano-Arevalo, D. R. Hernandez, W. G. Gonzalez, J. Miksovska, M. E. Ridgeway, M.A. Park, F. Fernandez-Lima, Anal. Chem., 86(20):10223-30, 2014. 


\begin{abstract}
Flavin adenine dinucleotide (FAD) is involved in important metabolic reactions where the biological function is intrinsically related to changes in conformation. In the present work, FAD conformational changes were studied in solution and in gas phase by measuring the fluorescence decay time and ion-neutral collision cross sections (CCS, in a trapped ion mobility spectrometer, TIMS) as a function of the solvent conditions (i.e., organic content) and gas-phase collisional partner (i.e., $\mathrm{N}_{2}$ doped with organic molecules). Changes in the fluorescence decay suggest that FAD can exist in four conformations in solution, where the abundance of the extended conformations increases with the organic content. TIMSMS experiments showed that FAD can exist in the gas phase as de-protonated $(\mathrm{M}=$ $\left.\mathrm{C}_{27} \mathrm{H}_{31} \mathrm{~N}_{9} \mathrm{O}_{15} \mathrm{P}_{2}\right)$ and protonated forms $\left(\mathrm{M}=\mathrm{C}_{27} \mathrm{H}_{33} \mathrm{~N}_{9} \mathrm{O}_{15} \mathrm{P}_{2}\right)$ and that multiple conformations (up to 12) can be observed as a function of the starting solution for the $[\mathrm{M}+\mathrm{H}]^{+}$and $[\mathrm{M}+\mathrm{Na}]^{+}$molecular ions. In addition, changes in the relative abundances of the gas-phase structures were observed from a "stack" to a "close" conformation when organic molecules were introduced in the TIMS cell as collision partners. Candidate structures optimized at the DFT/B3LYP/6-31G(d,p) were proposed for each IMS band and results showed that the most abundant IMS band corresponds to the most stable candidate structure. Solution and gas-phase experiments suggest that the driving force that stabilizes the different conformations is based on the interaction of the adenine and isoalloxazine rings that can be tailored by the "solvation" effect created with the organic molecules.
\end{abstract}




\section{INTRODUCTION}

Flavin adenine dinucleotide (FAD) is involved in multiple metabolic reactions. Its primary role is as a cofactor necessary for the activity of numerous flavoproteins, which play an important role in electron transport pathways in living systems like respiration ${ }^{1}$, photosynthesis ${ }^{2,3}$, DNA repair ${ }^{4,5}$, photoreceptors and nitrogen fixation ${ }^{6}$. Since Weber et al

${ }^{7}$ reported a weakness in the fluorescence of FAD in comparison with the fluorescence of free riboflavin, a number of studies have proposed the existence of at least two conformers for FAD in solution ${ }^{8,9}$. One "stack" conformer that exhibits a quenching of fluorescence, which presents a $\pi-\pi$ interaction between aromatic rings and intramolecular hydrogen bonds that stabilizes the adenine moiety and isoalloxazine ring, ${ }^{10,11}$ which might contribute to $80 \%$ of the molecules of FAD in solution. ${ }^{12}$ And an "open" conformer, which does not present quenching of the fluorescence. ${ }^{7,13}$. Even though the existence of an "open" and a "stack" conformation is generally accepted, little is known about the structural details and the conformational space of FAD. Studies on free riboflavin and the adenosine derivative 5'-bromo-5'-deoxyadenosine using crystallographic methods have shown an average structure of the $\pi-\pi$ systems. ${ }^{14}$ Different models for the interaction between the flavin and adenine moieties have been proposed based on NMR studies. ${ }^{11,15-18}$ However, all NMR studies presented the complication of intermolecular stacking between the flavin complexes at millimolar concentrations.

During the last decades, ion mobility spectrometry (IMS) combined with molecular dynamic simulations has proven to be a versatile technique for the analysis of intermediate and equilibrium structures of biomolecules enabling the correlation of ion-neutral, collision 
cross sections (CCS) with candidate structures. ${ }^{19-24}$ In particular, it has been shown that using soft ionization techniques (e.g., electrospray ionization, ESI ${ }^{25}$ ) the evaporative cooling of the solvent leads to a freezing of multiple conformations, which has permitted the study of the conformational space dependence on the solvent conditions (e.g., native vs denatured), bath gas collision partner and temperature. ${ }^{26-37}$ With the recent introduction of trapped ion mobility spectrometry coupled to mass spectrometry (TIMS-MS), ${ }^{38,39}$ we have shown that high mobility separations $(\mathrm{R}=100-250)$ can be routinely achieved. ${ }^{40} \mathrm{In}$ particular, we have shown that the study of biomolecules traditionally named "unstructured" by NMR and XRD is feasible due to the ability to measure multiple conformations at a given time and to perform kinetic studies of conformational interconversion as a way to elucidate folding/unfolding pathways. ${ }^{41,42}$

In the present work, the conformational space of FAD in solution phase and in gas phase as a function of the solution composition was studied using a combination of TIMS-MS, fluorescence time decay, and molecular dynamics (MD) simulations. We present evidence that $\mathrm{FAD}[\mathrm{M}+\mathrm{H}]^{+}$and $[\mathrm{M}+\mathrm{Na}]^{+}$molecular ions observed during ESI can be de-protonated and protonated, which leads to multiple stable conformations (totaling 12 IMS bands) whose relative abundance can be tailored by the solvent conditions and the gas-phase collision partner. Special attention was given to the interrelation between the MD and IMS data, and candidate structures for each IMS bands are proposed. 


\section{METHODS}

Materials and reagents

Flavin adenine dinucleotide disodium salt hydrate (F6625) powder was purchased from Sigma-Aldrich (St. Louis, MO) and used as received. All solvents and ammonium acetate salts used in these studies were analytical grade or better and purchased from Fisher Scientific (Pittsburg, PA). A stock solution was prepared in $10 \mathrm{mM}$ ammonium acetate and aliquots were diluted to a final concentration of 1,5 , and $10 \mu \mathrm{M}$ in 70:30, 50:50, and 30:70 (v/v) water-methanol/ethanol solutions. A Tuning Mix calibration standard (TuneMix, G24221A) was purchased from Agilent Technologies (Santa Clara, CA) and used as received. Details on the Tunemix structures (e.g., $\mathrm{m} / z=322 K_{0}=1.376 \mathrm{~cm}^{2} \mathrm{~V}^{-1} \mathrm{~s}^{-1}$ and $m / z=622 K_{0}=1.013 \mathrm{~cm}^{2} \mathrm{~V}^{-1} \mathrm{~s}^{-1}$ ) can be found elsewhere. ${ }^{40,43}$ All experiments were performed in triplicates.

Trapped Ion Mobility Spectrometry - Mass Spectrometry Separation

Details regarding the TIMS operation and specifics compared to traditional IMS can be

found elsewhere. ${ }^{38-42}$ Briefly, in TIMS mobility separation is based on holding the ions stationary using an electric field against a moving gas. The separation in a TIMS device can be described by the center of the mass frame using the same principles as in a conventional IMS drift tube. ${ }^{44}$ Since mobility separation is related to the number of ionneutral collisions (or drift time in traditional drift tube cells), the mobility separation in a TIMS device depends on the bath gas drift velocity, ion confinement and ion elution parameters. The reduced mobility, $K_{0}$, of an ion in a TIMS cell is described by: 
$K_{0}=\frac{v_{g}}{E}=\frac{A}{\left(V_{\text {elution }}-V_{\text {base }}\right)}$

where $\mathrm{vg}_{\mathrm{g}}, \mathrm{E}, \mathrm{V}_{\text {elution }}$ and $\mathrm{V}_{\text {base }}$ are the velocity of the gas, applied electric field, elution and base voltages, respectively. The constant A can be determined using calibration standards of known reduced mobilities. In TIMS operation, multiple geometric isomers/conformers are trapped simultaneously at different E values resulting from a voltage gradient applied across the TIMS tunnel. After thermalization, geometrical isomers/conformers are eluted by decreasing the electric field in stepwise decrements (referred to as the "ramp"). Each isomer/conformer eluting from the TIMS cell can be described by a characteristic voltage (i.e., $\mathrm{V}_{\text {elution }}-\mathrm{V}_{\text {base }}$ ). Eluted ions are then mass analyzed and detected by a maXis impact Q-ToF mass spectrometer (Bruker Daltonics Inc, Billerica, MA).

In a TIMS device, the total analysis time can be described as:

Total IMS time $=\mathrm{T}_{\text {trap }}+\left(\mathrm{V}_{\text {elution }} / \mathrm{V}_{\text {ramp }}\right) * \mathrm{~T}_{\text {ramp }}+\mathrm{ToF}=\mathrm{To}+\left(\mathrm{V}_{\text {elut }} / \mathrm{V}_{\text {ramp }}\right) * \mathrm{~T}_{\text {ramp }}$

where, $\mathrm{T}_{\text {trap }}$ is the thermalization/trapping time, $\mathrm{ToF}$ is the time after the mobility separation, and $\mathrm{V}_{\text {ramp }}$ and $\mathrm{T}_{\text {ramp }}$ are the voltage range and time required to vary the electric field, respectively. The elution voltage can be experimentally determined by varying the ramp time for a constant ramp voltage. This procedure also determines the time ions spend outside the separation region $\mathrm{T}_{\mathrm{o}}$ (e.g., ion trapping and time-of-flight).

The TIMS funnel is controlled using in-house software, written in National Instruments Lab VIEW, and synchronized with the maXis Impact Q-ToF acquisition program. ${ }^{38,39}$ 
TIMS separation was performed using nitrogen as a bath gas at $c a .300 \mathrm{~K}$, and the gas flow velocity was controlled by the pressure difference between entrance funnel $P_{1}=2.6 \mathrm{mbar}$, and the exit funnel $P_{2}=1.0$ mbar. $P_{1}$ and $P_{2}$ values were held constant for all experiments. Dopant additives were introduced at the entrance of the tunnel region of the TIMS analyzer and monitored with an external capacitance gauge from MKS instruments (Andover, MA). Methanol, ethanol and acetone were used as dopant additives and introduced through a $1 \mathrm{~mm}$ i.d. aperture at $10 \mathrm{mbar}$. The same RF (880 kHz and 200Vpp) was applied to all electrodes including the entrance funnel, the mobility separating section, and the exit funnel. An electrospray ionization source (ESI Apollo II design, Bruker Daltonics, Inc., MA) was used for all the analyses. The TIMS cell was operated using a fill/trap/ramp/wait sequence of $10 / 10 / 50-500 / 50 \mathrm{~ms}$. Average mobility resolution at 10/10/500/50 was 160190.

Reduced mobility values $\left(\mathrm{K}_{0}\right)$ were correlated with $\operatorname{CCS}(\Omega)$ using the equation:

$$
\Omega=\frac{(18 \pi)^{1 / 2}}{16} \frac{z}{\left(k_{B} T\right)^{1 / 2}}\left[\frac{1}{m_{I}}+\frac{1}{m_{b}}\right]^{1 / 2} \frac{1}{K_{0}} \frac{1}{N^{*}}
$$

where $\mathrm{z}$ is the charge of the ion, $\mathrm{k}_{\mathrm{B}}$ is the Boltzmann constant, $\mathrm{N}^{*}$ is the number density and $m_{I}$ and $m_{b}$ refer to the masses of the ion and bath gas, respectively. ${ }^{44}$ 
Fluorescence Decay Analysis

Fluorescence decay experiments were performed on FAD as a function of the solvent conditions using a ChronosFD spectrofluorometer (ISS, Champaign, IL) in the frequency domain mode. A $10 \mu \mathrm{M}$ FAD solution was excited using a $470 \mathrm{~nm}$ laser diode and fluorescent emission was collected using a 500nm and $650 \mathrm{~nm}$ long and short pass filters, respectively (Andover, Salem, NH). A rhodamine B water solution was used for lifetime calibration $(\tau=1.7 \mathrm{~ns})$. ${ }^{45}$ Polarizers were set at magic angle configuration. The fluorescence decay lifetime were recovered by a non-linear fit of the data using a triple exponential decay using GlobalWe software (Laboratory of Fluorescence Dynamics, Irvine, CA).

Theoretical Calculations

A pool of candidate structures were proposed for each molecular ion observed in the TIMSMS experiments. The initial pool of candidate structures was obtained using serial molecular dynamics simulations of annealing and geometry optimization cycles in a NVT thermostat (equivalent to the approach described in ref ${ }^{31}$ ) using AMBER03 force field in YASARA software. In particular, the NVT thermostat was set to recreate the TIMS cell experiment; the simulation box contained the molecular ion of interest with bath and dopant gas molecules. Final structures were optimized at the DFT/B3LYP/6-31G(d,p) level using Gaussian software. ${ }^{46}$ Vibrational frequencies were calculated to guarantee that the optimized structures correspond to a real minima in the energy space and zero-point energy corrections were applied to calculate the relative stability between the structures. Theoretical ion-neutral collision cross sections were calculated using MOBCAL version 
for helium ${ }^{47,48}$ and nitrogen ${ }^{49-50}$ as a bath gas at ca. $300 \mathrm{~K}$. It should be noted that MOBCAL version for nitrogen was used assuming the similarity of the molecules to those used to develop the Lennard-Jones potential at $300 \mathrm{~K}$ in ref ${ }^{49-50}$; for other molecules alternatives methods are encouraged ${ }^{51}$ Partial atomic charges were calculated using the Merz-Singh-Kollman scheme constrained to the molecular dipole moment. ${ }^{52,53}$ All optimized geometries and partial atomic charges used in the MOBCAL input files are provided in the supporting information.

\section{RESULTS AND DISCUSSION}

The MS analysis of FAD shows that four molecular ions are produced by the ESI source, independent of the water: organic content ratio (Figure 2.1). The most abundant $[\mathrm{M}+\mathrm{H}]^{+}$ and $[\mathrm{M}+\mathrm{Na}]^{+}$molecular ions correspond to the protonated form of FAD $\left(\mathrm{M}=\mathrm{C}_{27} \mathrm{H}_{33} \mathrm{~N}_{9} \mathrm{O}_{15} \mathrm{P}_{2}\right)$, while similar molecular ions were observed for the deprotonated form $\left(\mathrm{M}=\mathrm{C}_{27} \mathrm{H}_{31} \mathrm{~N}_{9} \mathrm{O}_{15} \mathrm{P}_{2}\right)$. This result is consistent with previously reported MS experiments (KNA00612 record from www.massbank.jp). Taking advantage of the high resolution of the MS spectrometer $(\mathrm{R}>30-40 \mathrm{k})$, mass signals were isolated $(\Delta \mathrm{m} / \mathrm{z}<1 \mathrm{Da}$, monoisotopic peak) and mobility experiments were performed for each molecular ion as a function of the solvent condition and bath gas composition.

Mobility experiments were performed as a function of the organic content in the starting solutions (e.g., water: ethanol/methanol content) using nitrogen as a bath gas (Figure 2.2). Inspection of the mobility spectrum showed that multiple conformations exist as a function of the solvent conditions (label A-L). Ion-neutral CCS were determined for all molecular 
ions observed using Tuning Mix species as external calibrants (Table 1). Major differences in the relative abundances were not observed as a function of the starting solution (e.g., organic content) and the IMS trapping time. This suggests that all structures formed during the ESI process (labeled A-L) are stable in the TIMS experiments time scale (50-2000 ms).

The conformational heterogeneity of FAD in solution was characterized by measuring the FAD lifetime in water: ethanol/methanol mixtures using frequency domain fluorescence spectroscopy (Figure 2.3). The data were analyzed using triple exponential decay model and the results are summarized in Table 2. Three distinct components were resolved: a fast component of $\sim 270 \mathrm{ps}$ and two nanosecond components with the lifetimes of $2.43 \mathrm{~ns}$ and $4.6 \mathrm{~ns}$ in water. The observed lifetimes are in agreement with previous reports of FAD emission lifetimes in water and water-ethanol mixtures. ${ }^{54-55}$ The fast decaying component of $\sim 270 \mathrm{ps}$ is attributed to a "closed" FAD conformation with relatively weak interactions between the isoalloxazine and adenine ring that lead to a less efficient intramolecular transfer between the hetero-aromatic groups. ${ }^{7,13}$ The nanosecond lifetimes of $\sim 2.5 \mathrm{~ns}$ and $\sim 4.6 \mathrm{~ns}$ correspond to the "partially open" and "open" FAD conformation, respectively. In the "open" conformation, the distance between the isoalloxazine and adenine ring was proposed to be approximately $16 \AA$, preventing an efficient quenching of the flavine emission. ${ }^{56}$ An additional lifetime of $\sim 10 \mathrm{ps}$ (not resolved in our measurements) was identified in femtosecond fluorescence lifetime studies and attributed to a "stack" conformation with the intramolecular distance between the hetero-aromatic groups of $\sim 4$ A. ${ }^{54}$ Inspection of Figure 2.3 and Table 2.2 shows that the increase in the organic content (i.e., methanol/ethanol) leads to the increase in the fraction of the open conformation that 
is characterized by $\sim 4.6 \mathrm{~ns}$ lifetime; this is in good agreement with previous experiments where the increase in the dielectric constant of medium altered the population of "stack" and "open" conformations of FAD. ${ }^{54,56}$

Candidate structures were proposed for IMS bands A-L (Figure 2.4). For the $[\mathrm{M}+\mathrm{H}]^{+}$and $[\mathrm{M}+\mathrm{Na}]^{+}$molecular ions observed of the deprotonated $\left(\mathrm{M}=\mathrm{C}_{27} \mathrm{H}_{31} \mathrm{~N}_{9} \mathrm{O}_{15} \mathrm{P}_{2}\right)$ and protonated $\left(\mathrm{M}=\mathrm{C}_{27} \mathrm{H}_{33} \mathrm{~N}_{9} \mathrm{O}_{15} \mathrm{P}_{2}\right)$ forms of $\mathrm{FAD}$, the lowest energy structure for each molecular ion form corresponds to most abundant IMS band; that is, the lowest energy structures are the most thermodynamically stable and appear with the larger relative abundance in the IMS spectra. Both deprotonated forms of FAD show more IMS bands than the protonated forms. Inspection of the $[\mathrm{M}+\mathrm{H}]^{+}$molecular ion form of the deprotonated $\left(\mathrm{C}_{27} \mathrm{H}_{31} \mathrm{~N}_{9} \mathrm{O}_{15} \mathrm{P}_{2}\right)$ FAD form shows that main differences between the candidate structures proposed for the five IMS bands (A-E) is on the distance and orientation between the adenine and isoalloxazine rings, where the de-protonation of the phosphate group near the adenine generates a resonance structure that stabilizes the $\pi-\pi$ system between both rings. The $[\mathrm{M}+\mathrm{Na}]^{+}$ molecular ions of the deprotonated $\left(\mathrm{C}_{27} \mathrm{H}_{31} \mathrm{~N}_{9} \mathrm{O}_{15} \mathrm{P}_{2}\right)$ FAD (F-H) form also showed the same orientation between both aromatic systems with an additional stabilization of the sodium ion present in the adduct. Moreover, the inspection of the $[\mathrm{M}+\mathrm{H}]^{+}$molecular ion form of the protonated $\left(\mathrm{C}_{27} \mathrm{H}_{33} \mathrm{~N}_{9} \mathrm{O}_{15} \mathrm{P}_{2}\right)$ FAD form shows that main differences between the candidate structures proposed for the (I-J) IMS bands is on the interaction between the ring systems without the stabilization provided by the resonance structure of the phosphate groups. The $[\mathrm{M}+\mathrm{Na}]^{+}$molecular ions of the protonated $\left(\mathrm{C}_{27} \mathrm{H}_{33} \mathrm{~N}_{9} \mathrm{O}_{15} \mathrm{P}_{2}\right)$ FAD form (K-L) also encounter the destabilization of the sodium ion present in the adduct. Inspection of the 
distances between the rings (4-6 $\AA$ ) in the proposed candidate structures showed a good agreement with the solution experiments for the "stack" and "close" conformations, as well as previous reports. ${ }^{54,56} \mathrm{~A}$ candidate structure for the "open" conformation will result in a CCS of $320 \AA^{2}$ (see structure in the supplemental information) but no IMS band was experimentally observed. The later suggest that solvation effects may equilibrate the "open" conformation in solution, while in the gas-phase the ring interaction dominates and stabilizes in the "stack" and "close" conformations. Since the molar fraction of the "close" FAD conformation of $\sim 270$ ps lifetime is higher in the ethanol: water mixture compare to the methanol-ammonium acetate mixture or methanol-water mixture, we attribute the fraction of FAD with 270 ps lifetime to the larger CCSs conformations identified in IMS measurements (e.g., band H). Despite that different molecular ions are observed, inspection of the IMS spectra shows that mainly five IMS bands are detected (considering the expected CCS small shift between $[\mathrm{M}+\mathrm{H}]^{+}$and $[\mathrm{M}+\mathrm{Na}]^{+}$ions) but a direct correlation from the number of IMS bands to the number of fluorescence decay times is not possible. Moreover, the decay times can be related to the distances between the isoalloxazine and adenine ring. Inspection of the distances from the proposed candidate structures shows three main groups: $4.3 \AA$ (conformer A), 4.8-5.6 $\AA$ (conformer B, C, D, F, G, H, I, J, K and L), and $5.9 \AA$ (conformer E). That is, the IMS experiments suggest that at least three decay times should be expected; however, differences between the distances of the three group of conformers can be too small to be resolved in life time measurements. ${ }^{54,56}$

The "solvation" and organic context effect on the number of FAD conformations was studied in the TIMS cell by using dopant gas additives (Figure 2.5). That is, each molecular 
ion conformation is isolated in the TIMS cell, and by introducing the dopant gas, we studied the influence of the collisional partner in the FAD conformational space in a single molecular ion - dopant molecule fashion. Inspection of Figure 5 shows that the interaction with the collision partner (electrostatic in nature) results in changes in the relative abundances of the "stack" and "close" FAD conformers in the absence and presence of the dopant gas additives. That is, experiments suggest that at the molecular level, the interaction with the organic molecules can induce the transition from "stack" to "close" conformations. Previous IMS studies have shown that different degrees of solvation are attainable as a function of the IMS experimental conditions and can be used to: i) increase IMS separation (e.g., drift tube, high field and differential IMS analyzers ${ }^{57-60}$ ), and ii) for structural assignments (e.g., determination of the solution state structures ${ }^{28,29,36}$ ). Polar molecules (e.g., methanol, ethanol and acetonitrile) interact with ions very strongly via iondipole interactions (since they have a permanent dipole moment). Although changes in the IMS profile occurred with the dopant introduction (i.e., structure $\mathrm{L}$ and $\mathrm{K}$ relative abundances), the transition into the "open" conformation was not observed. The later suggest that a higher abundance of dopant molecules with respect to the bath gas is necessary to reach the FAD "open" state. Comparison between the solution and gas-phase results suggest that the FAD solution state distribution can be preserved in the gas-phase and gas-phase conformation-friendly conditions can be induced using dopant gases. Moreover, in both solution and gas-phase, FAD three-dimensional structures are determined by the interplay between intramolecular interactions (e.g., $\pi$ - $\pi$ system formed by the adenine and isoalloxazine rings) and interactions with the surrounding solvent/gas molecules. The workflow described here (TIMS-MS combined with MD using gas 
dopants) provides a powerful tool for the investigation of the gas phase "solvation" state of molecular ions.

\section{CONCLUSIONS}

In the current study, TIMS-MS combined fluorescence time decay and theoretical calculations was used to study FAD conformational space. Gas-phase experimental results showed that $[\mathrm{M}+\mathrm{H}]^{+}$and $[\mathrm{M}+\mathrm{Na}]^{+}$molecular ions are observed during ESI for the

deprotonated $\left(\mathrm{C}_{27} \mathrm{H}_{31} \mathrm{~N}_{9} \mathrm{O}_{15} \mathrm{P}_{2}\right)$ and protonated $\left(\mathrm{C}_{27} \mathrm{H}_{33} \mathrm{~N}_{9} \mathrm{O}_{15} \mathrm{P}_{2}\right)$ FAD forms. For the first time, CCSs of 12 FAD conformations found by IMS experiments are reported and compared with theoretical calculations of candidate structures that correspond to the "stack" and "close" conformations identified in solution by fluorescence lifetime measurements. The abundance of each conformer was consistent with their relative stability; that is, the larger intensity observed for a conformer corresponds to the most stable conformer. The examination of the conformational space generated by the candidate structures shows that the main motif that defines FAD conformational space is the interaction between the isoalloxazine and adenine groups. It was shown that the use of dopants in the TIMS cell permits the investigation of single molecular ion- dopant molecule interaction and can be used to study the gas-phase "solvation" state of biological molecules.

\section{ACKNOWLEDGMENTS}

This work was supported by the National Institute of Health (Grant No. R00GM106414) and a FFL Bruker Daltonics, Inc. fellowship. The authors wish to acknowledge Dr. Desmond Kaplan from Bruker Daltonics, Inc. for the development of TIMS acquisition 
software. The authors would like to thank Dr. Alexander Mebel (Florida International University) and Dr. Matthew Bush (University of Washington) for helpful discussions during the theoretical calculations and MOBCAL for nitrogen calculations, respectively. 
Figure 2.1 Structure and typical MS spectra for the deprotonated $\left(\mathrm{M}=\mathrm{C}_{27} \mathrm{H}_{31} \mathrm{~N}_{9} \mathrm{O}_{15} \mathrm{P}_{2}\right)$ and protonated $\left(\mathrm{M}=\mathrm{C}_{27} \mathrm{H}_{33} \mathrm{~N}_{9} \mathrm{O}_{15} \mathrm{P}_{2}\right)$ FAD forms. Monoisotopic peaks of the $[\mathrm{M}+\mathrm{H}]^{+}$and $[\mathrm{M}+\mathrm{Na}]^{+}$molecular ions used in the mobility analysis are highlighted with a dashed rectangle.

A

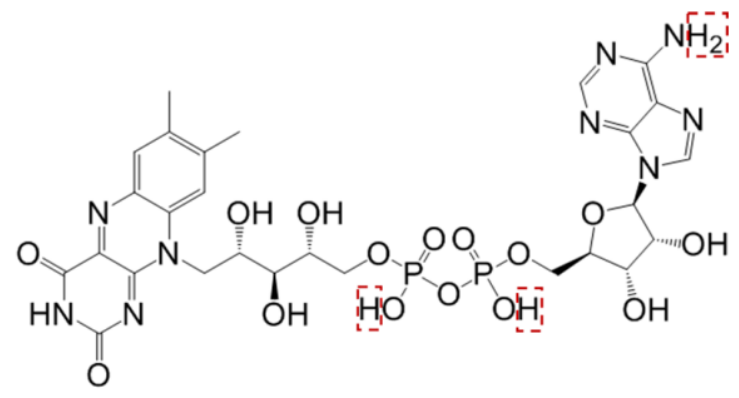

B

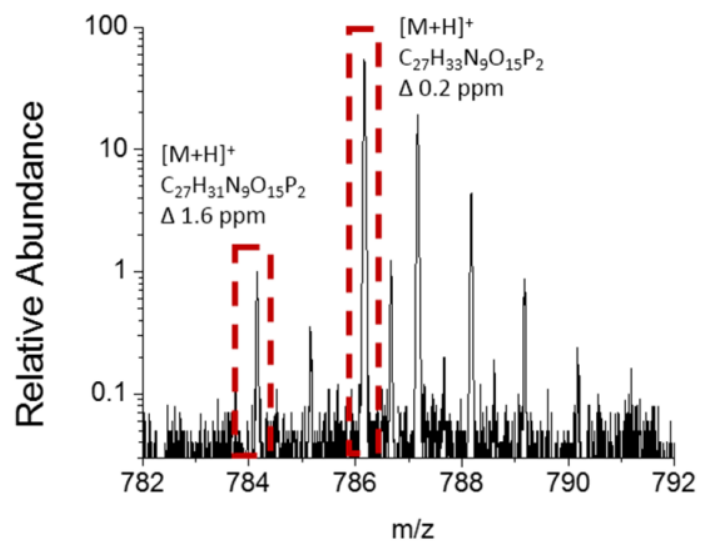

C

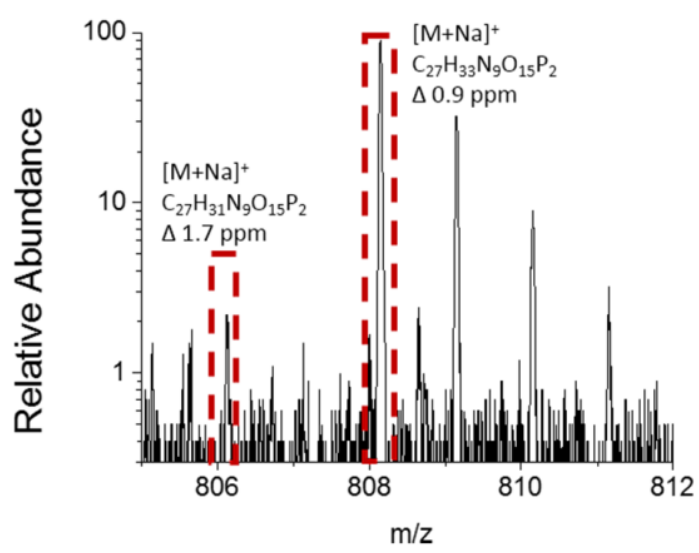


Figure 2.2 Typical TIMS spectra as a function of the organic content for the $[\mathrm{M}+\mathrm{H}]^{+}$and $[\mathrm{M}+\mathrm{Na}]^{+}$molecular ions of the deprotonated $\left(\mathrm{M}=\mathrm{C}_{27} \mathrm{H}_{31} \mathrm{~N}_{9} \mathrm{O}_{15} \mathrm{P}_{2}\right)$ and protonated $\left(\mathrm{M}=\mathrm{C}_{27} \mathrm{H}_{33} \mathrm{~N}_{9} \mathrm{O}_{15} \mathrm{P}_{2}\right)$ FAD forms.

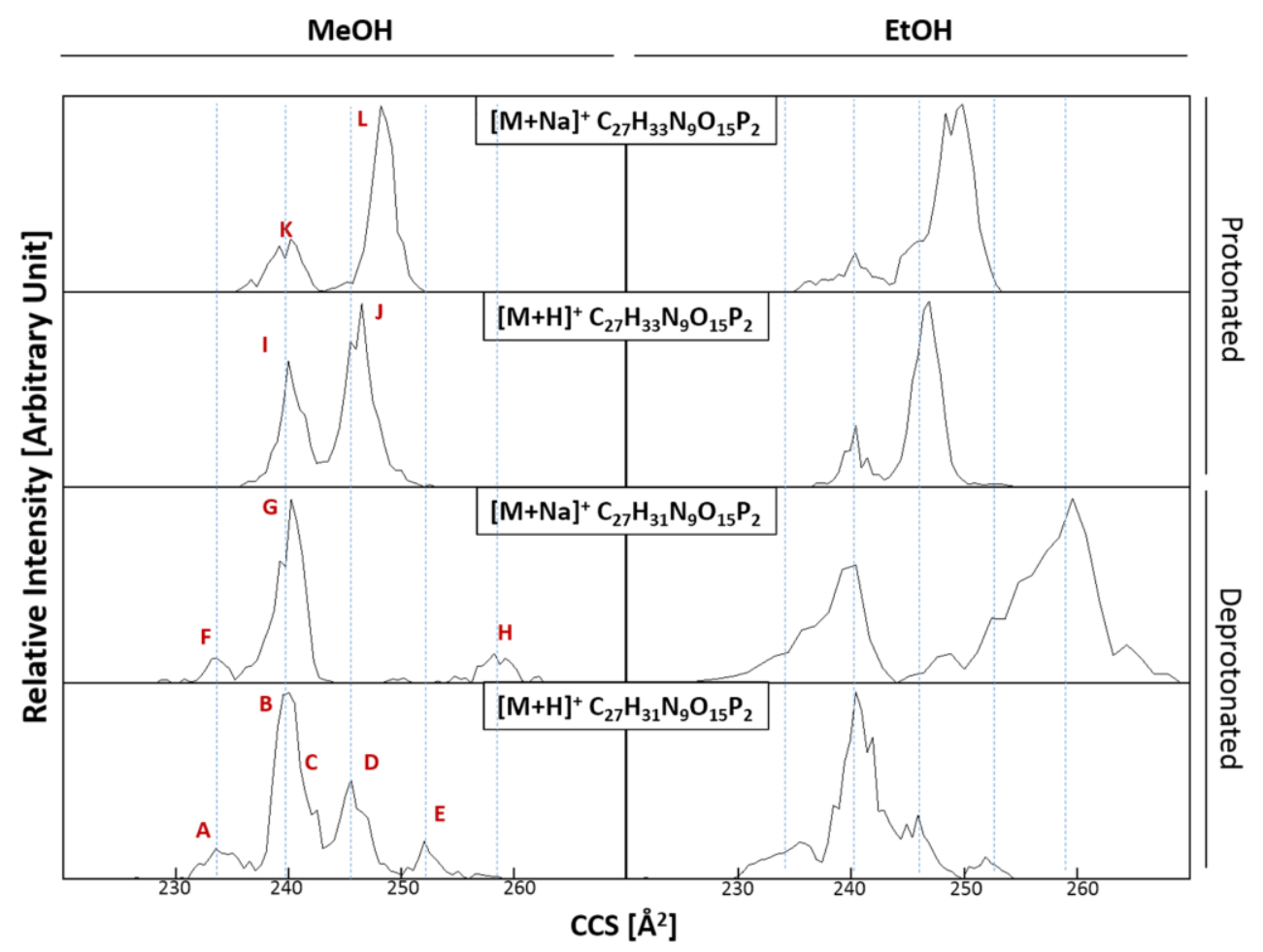


Figure 2.3 Frequency domain phase delay (solid squares) and modulation ratio (open circles) of $10 \mu \mathrm{M}$ FAD in mixture of a) ethanol: water, b) methanol: water and c) $10 \mu \mathrm{M}$ FAD in mixture of ammonium acetate buffer: water as a function of the volume ratio of 0:100 (black line), 30:70 (red line), (50:50) green line and 70:30 (blue line). Solid lines represent the best to the data using a triple exponential decay.

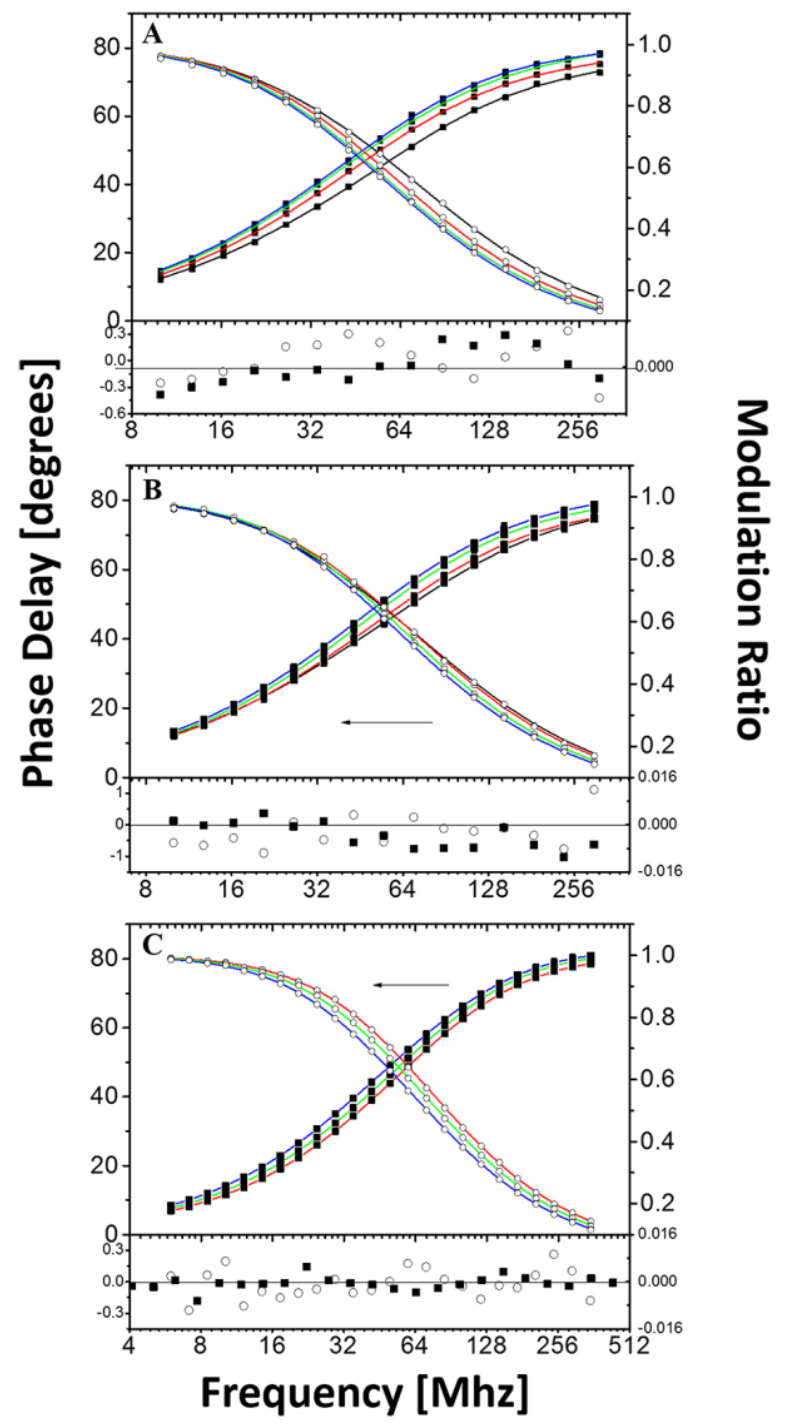


Figure 2.4 Candidate structures for the IMS bands observed for the $[\mathrm{M}+\mathrm{H}]^{+}$and $[\mathrm{M}+\mathrm{Na}]^{+}$ molecular ions of the deprotonated $\left(\mathrm{M}=\mathrm{C}_{27} \mathrm{H}_{31} \mathrm{~N}_{9} \mathrm{O}_{15} \mathrm{P}_{2}\right)$ and protonated $\left(\mathrm{M}=\mathrm{C}_{27} \mathrm{H}_{33} \mathrm{~N}_{9} \mathrm{O}_{15} \mathrm{P}_{2}\right)$ FAD forms.

A

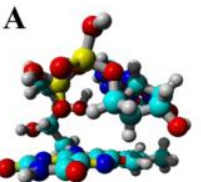

$[\mathrm{M}+\mathrm{H}]^{+} \mathrm{C}_{27} \mathrm{H}_{31} \mathrm{~N}_{9} \mathrm{O}_{15} \mathrm{P}_{2}$ $\mathrm{d}=4.318 \AA, \mathrm{CCS}=238.31 \AA^{2}$

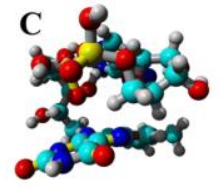

$[\mathrm{M}+\mathrm{H}]^{+} \mathrm{C}_{27} \mathrm{H}_{31} \mathrm{~N}_{9} \mathrm{O}_{15} \mathrm{P}$ $\mathrm{d}=5.122 \AA, \mathrm{CCS}=244.85 \AA^{2}$

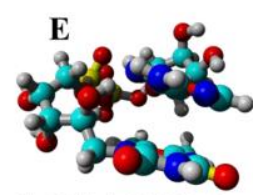

$[\mathrm{M}+\mathrm{H}]^{+} \mathrm{C}_{27} \mathrm{H}_{31} \mathrm{~N}_{9} \mathrm{O}_{15} \mathrm{P}_{2}$ $\mathrm{d}=5.915 \AA, \mathrm{CCS}=252.79 \AA^{2}$

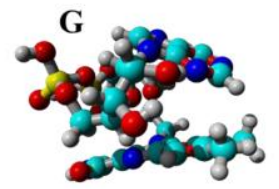

$[\mathrm{M}+\mathrm{Na}]^{+} \mathrm{C}_{27} \mathrm{H}_{31} \mathrm{~N}_{9} \mathrm{O}_{15} \mathrm{P}_{2}$ $\mathrm{d}=5.570 \AA, \mathrm{CCS}=241.53 \AA^{2}$

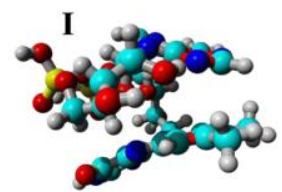

$[\mathrm{M}+\mathrm{H}]^{+} \mathrm{C}_{27} \mathrm{H}_{33} \mathrm{~N}_{9} \mathrm{O}_{15} \mathrm{P}_{2}$ $\mathrm{d}=4.969 \AA, \mathrm{CCS}=258.08 \AA^{2}$

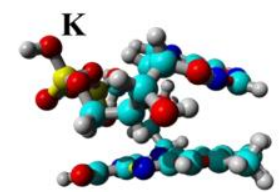

$[\mathrm{M}+\mathrm{Na}]^{+} \mathrm{C}_{27} \mathrm{H}_{33} \mathrm{~N}_{9} \mathrm{O}_{15} \mathrm{P}_{2}$
B
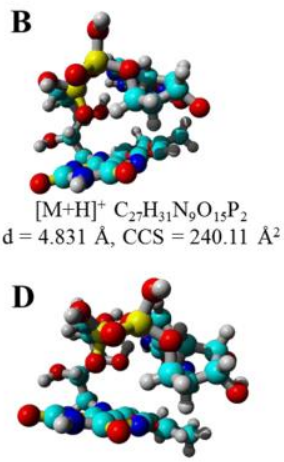

$[\mathrm{M}+\mathrm{H}]^{+} \mathrm{C}_{27} \mathrm{H}_{31} \mathrm{~N}_{9} \mathrm{O}_{15} \mathrm{P}$ $\mathrm{d}=5.244 \AA, \mathrm{CCS}=247.92 \AA^{2}$

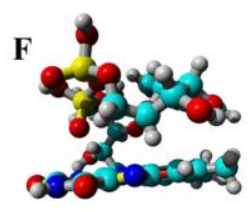

$[\mathrm{M}+\mathrm{Na}]^{+} \mathrm{C}_{27} \mathrm{H}_{31} \mathrm{~N}_{9} \mathrm{O}_{15} \mathrm{P}_{2}$ $\mathrm{d}=5.204 \AA, \mathrm{CCS}=234.28 \AA^{2}$
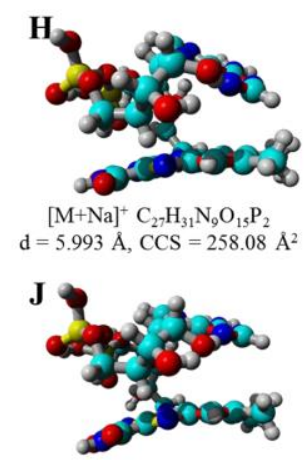

$[\mathrm{M}+\mathrm{H}]^{+} \mathrm{C}_{27} \mathrm{H}_{33} \mathrm{~N}_{9} \mathrm{O}_{15} \mathrm{P}_{2}$ $\mathrm{d}=5.102 \AA, \mathrm{CCS}=247.51 \AA^{2}$

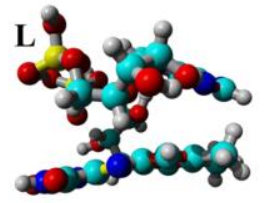

$[\mathrm{M}+\mathrm{Na}]^{+} \mathrm{C}_{27} \mathrm{H}_{33} \mathrm{~N}_{9} \mathrm{O}_{15} \mathrm{P}_{2}$ 
Figure 2.5 Typical IMS spectra of the $[\mathrm{M}+\mathrm{Na}]^{+}$ions of the protonated FAD form $\left(\mathrm{M}=\mathrm{C}_{27} \mathrm{H}_{33} \mathrm{~N}_{9} \mathrm{O}_{15} \mathrm{P}_{2}\right)$ as a function of the bath gas conditions. Notice the variation of the relative abundances of IMS bands as a function of the bath gas composition using ethanol, methanol and acetonitrile as additives in the TIMS mobility cell.

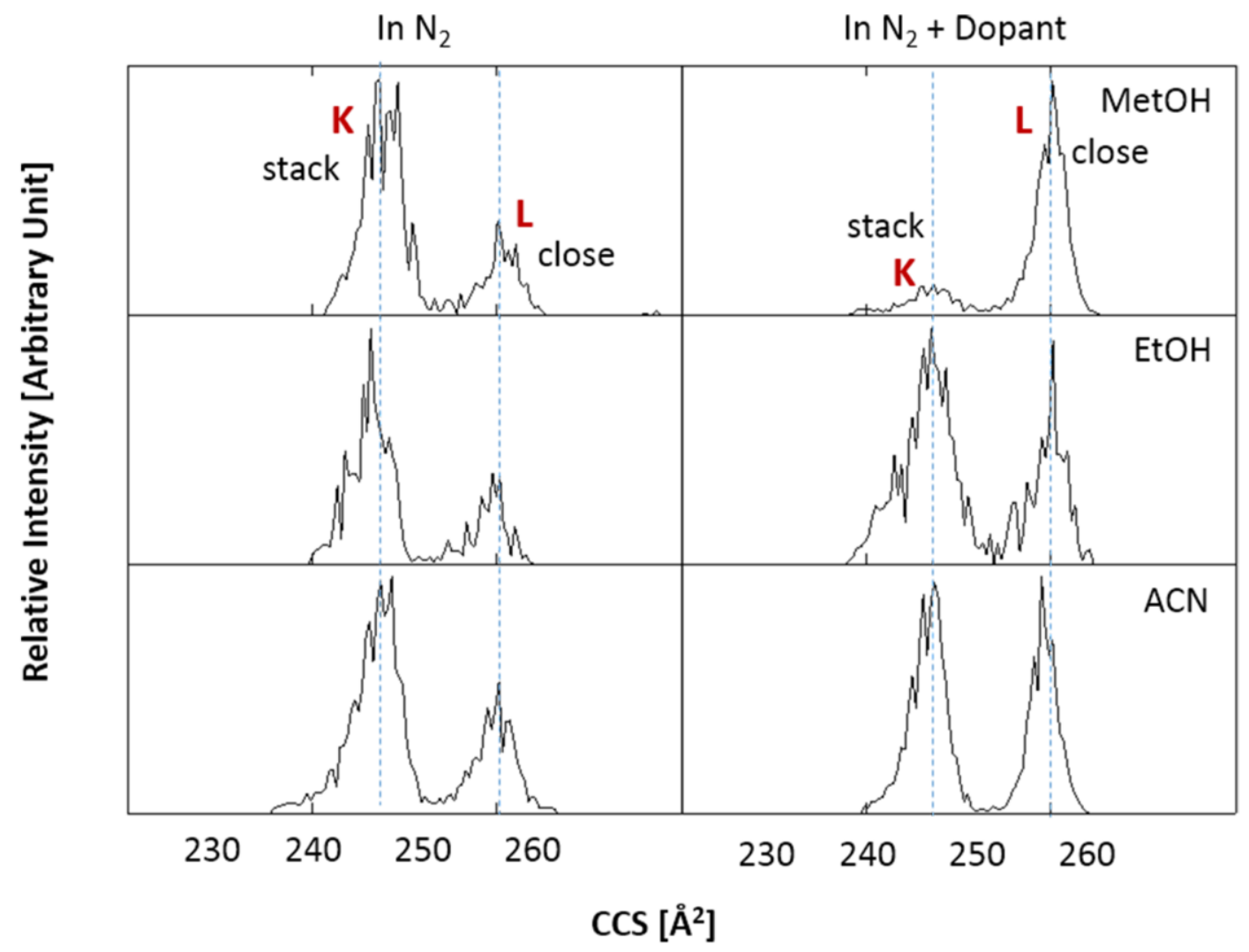


Table 2.1 Experimental and theoretical ion-neutral collision cross section for the $[\mathrm{M}+\mathrm{H}]^{+}$ and $[\mathrm{M}+\mathrm{Na}]^{+}$molecular ions of the deprotonated $\left(\mathrm{M}=\mathrm{C}_{27} \mathrm{H}_{31} \mathrm{~N}_{9} \mathrm{O}_{15} \mathrm{P}_{2}\right)$ and protonated $\left(\mathrm{M}=\mathrm{C}_{27} \mathrm{H}_{33} \mathrm{~N}_{9} \mathrm{O}_{15} \mathrm{P}_{2}\right)$ FAD forms. Energies were calculated at level B3LYP/6-31G(d,P) and shown relative to the most stable isomer. The distances (d) between the isoalloxazine and adenine ring are shown for each conformation.

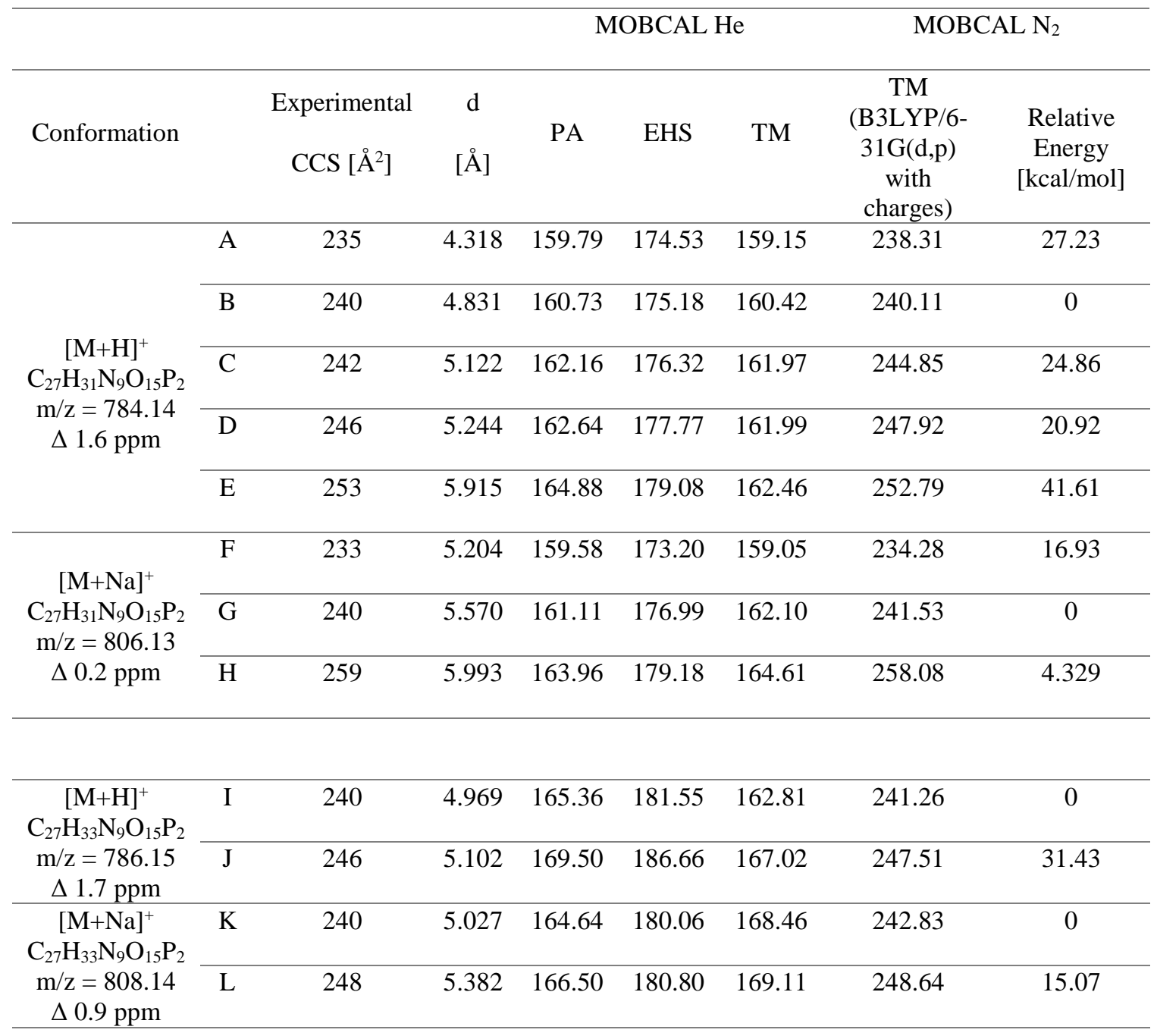


Table 2.2 Fluorescence decay parameters recovered for FAD in ethanol-water, methanolwater, and methanol-ammonium acetate mixtures.

\begin{tabular}{lccccccc}
\hline & $\alpha_{0}$ & $\tau_{0}(\mathrm{~ns})$ & $\alpha_{1}$ & $\tau_{1}(\mathrm{~ns})$ & $\alpha_{2}$ & $\tau_{2}(\mathrm{~ns})$ & $\chi^{2}$ \\
\hline Water & 0.28 & 0.27 & 0.50 & 2.43 & 0.23 & 4.62 & 1.02 \\
\hline $\begin{array}{c}\text { EtOH-Water (\%) } \\
\text { 30-70 }\end{array}$ & 0.27 & 0.27 & 0.41 & 2.43 & 0.32 & 4.62 & 0.58 \\
50-50 & 0.21 & & 0.31 & & 0.49 & & 0.33 \\
$70-30$ & 0.22 & & 0.26 & & 0.52 & & 1.32 \\
MetOH-Water (\%) & & & & & & & \\
\hline 30-70 & 0.19 & 0.36 & 0.71 & 2.45 & 0.09 & 4.25 & 1.33 \\
$50-50$ & 0.22 & & 0.68 & & 0.10 & & 2.84 \\
$70-30$ & 0.20 & & 0.66 & & 0.14 & & 1.18
\end{tabular}

MetOH-Ammonium

Acetate (\%)

\begin{tabular}{llllllll}
$30-70$ & 0.17 & 0.29 & 0.73 & 2.75 & 0.10 & 5.06 & 0.33 \\
$50-50$ & 0.15 & & 0.67 & & 0.18 & & 0.68 \\
$70-30$ & 0.13 & & 0.56 & & 0.31 & & 0.67 \\
\hline
\end{tabular}

Parameters recovered using a triple exponential decay model. Decay lifetimes were set as linked variables. Errors of the recovered values not shown but did not exceed $17 \%$ of their values. 


\section{LIST OF REFERENCES}

(1) Amino, H.; Osanai, A.; Miyadera, H.; Shinjyo, N.; Tomitsuka, E.; Taka, H.; Mineki, R.; Murayama, K.; Takamiya, S.; Aoki, T.; Miyoshi, H.; Sakamoto, K.; Kojima, S.; Kita, K. Mol. Biochem. Parasit. 2003, 128, 175.

827.

(2) Braatsch, S.; Gomelsky, M.; Kuphal, S.; Klug, G. Mol. Microbiol. 2002, 45,

(3) Bauer, C.; Elsen, S.; Swem, L. R.; Swem, D. L.; Masuda, S. Philos. T. R. Soc. B. 2003, 358, 147.

(4) MacFarlane, A. W. t.; Stanley, R. J. Biochemistry 2003, 42, 8558-8568.

(5) van der Horst, M. A.; Hellingwerf, K. J. Accounts. Chem. Res. 2004, 37, 13.

(6) Cosseau, C.; Garnerone, A. M.; Batut, J. Mol. Plant. Microbe. In. 2002, 15, 598.

(7) Weber, G. Biochem. J. 1950, 47, 114.

(8) Barrio, J. R.; Tolman, G. L.; Leonard, N. J.; Spencer, R. D.; Weber, G. P. Natl. Acad. Sci. USA. 1973, 70, 941.

(9) Weber, G.; Tanaka, F.; Okamoto, B. Y.; Drickamer, H. G. Proc. Natl. Acad. Sci. USA. 1974, 71, 1264. $561-566$.

(10) Grininger, M.; Seiler F.;Zeth, K.; Oesterhelt, D.; J. Mol. Biol. 2006, 364,

(11) Raszka, M.; Kaplan, N. O. P. Natl. Acad. Sci. USA. 1974, 71, 4546.

(12) van den Berg, P. A. W.; Feenstra, K. A.; Mark, A. E.; Berendsen, H. J. C.; Visser, A. J. W. G. J. Phys. Chem. US. B. 2002, 106, 8858. 15254.

(13) Nakabayashi, T.; Islam, M. S.; Ohta, N. J. Phys. Chem. US. B. 2010, 114,

(14) Voet, D.; Rich, A. Proc. Natl. Acad. Sci. USA. 1971, 68, 1151.

(15) Sarma, R. H.; Dannies, P.; Kaplan, N. O. Biochemistry-US. 1968, 7, 4359.

(16) Sarma, R. H.; Mynott, R. J. J. Am. Chem. Soc. 1973, 95, 1641.

(17) Kainosho, M.; Kyogoku, Y. Biochemistry-US. 1972, 11, 741. 

5656.

(18) Kotowycz, G.; Teng, N.; Klein, M. P.; Calvin, M. J. Biol. Chem. 1969, 244,

(19) Valentine, S. J.; Counterman, A. E.; Clemmer, D. E. J. Am. Soc. Mass. Spectrom. 1999, 10, 1188.

(20) Kanu, A. B.; Dwivedi, P.; Tam, M.; Matz, L.; Hill, H. H. J. Mass Spectrom. $2008,43,1$.

(21) Tao, L.; McLean, J. R.; McLean, J. A.; Russell, D. H. J. Am. Soc. Mass. Spectrom. 2007, 18, 1232.

(22) Ruotolo, B. T.; Benesch, J. L. P.; Sandercock, A. M.; Hyung, S.-J.; Robinson, C. V. Nat. Protocols. 2008, 3, 1139.

(23) Fernandez-Lima, F. A.; Blase, R. C.; Russell, D. H. Int. J. Mass Spectrom. 2010, 298, 111.

(24) Beveridge, R.; Chappuis, Q.; Macphee, C.; Barran, P. Analyst. 2013, 138, 32. $246,64$.

(25) Fenn, J.; Mann, M.; Meng, C.; Wong, S.; Whitehouse, C. Science. 1989,

(26) Wyttenbach, T.; von Helden, G.; Bowers, M. T. J. Am. Chem. Soc. 1996, $118,8355$.

(27) Li, J.; Taraszka, J. A.; Counterman, A. E.; Clemmer, D. E. Int. J. Mass Spectrom. 1999, 185-187, 37.

(28) Kohtani, M.; Jarrold, M. F. J. Am. Chem. Soc. 2002, 124, 11148.

(29) Kohtani, M.; Breaux, G. A.; Jarrold, M. F. J. Am. Chem. Soc. 2004, 126, 1206.

(30) Hyung, S.-J.; Robinson, C. V.; Ruotolo, B. T. Chem. Biol. 2009, 16, 382.

(31) Fernandez-Lima, F. A.; Wei, H.; Gao, Y. Q.; Russell, D. H. J. Phys. Chem. A. 2009, 113, 8221 .

(32) Bush, M. F.; Hall, Z.; Giles, K.; Hoyes, J.; Robinson, C. V.; Ruotolo, B. T. Anal. Chem. 2010, 82, 9557.

(33) Pierson, N. A.; Chen, L.; Valentine, S. J.; Russell, D. H.; Clemmer, D. E. J. Am. Chem. Soc. 2011, 133, 13810. 
(34) Zhong, Y.; Hyung, S.-J.; Ruotolo, B. T. Expert Rev. Proteomics. 2012, 9, 47.

(35) Beveridge, R.; Chappuis, Q.; Macphee, C.; Barran, P. Analyst. 2013, 138, 32.

(36) Silveira, J. A.; Fort, K. L.; Kim, D.; Servage, K. A.; Pierson, N. A.; Clemmer, D. E.; Russell, D. H. J. Am. Chem. Soc. 2013, 135, 19147.

(37) Chen, L.; Chen, S. H.; Russell, D. H. Anal. Chem. 2013, 85, 7826.

(38) Fernandez-Lima, F. A.; Kaplan, D. A.; Suetering, J.; Park, M. A. Int. J. Ion Mobil. Spectrom. 2011, 14, 93.

(39) Fernandez-Lima, F. A.; Kaplan, D. A.; Park, M. A. Rev. Sci. Instr. 2011, $82,126106$.

(40) Hernandez, D. R.; DeBord, J. D.; Ridgeway, M. E.; Kaplan, D. A.; Park, M. A.; Fernandez-Lima, F. Analyst. 2014, 139, 1913.

(41) Schenk, E. R.; Ridgeway, M. E.; Park, M. A.; Leng, F.; Fernandez-Lima, F. Anal. Chem. 2014, 86, 1210.

(42) Schenk, E. R.; Mendez, V.; Landrum, J. T.; Ridgeway, M. E.; Park, M. A.; Fernandez-Lima, F. Anal. Chem. 2014, 86, 2019.

(43) Flanagan, L. A.; Patent, U. S., Ed.; Hewlett-Packard Company (Palo Alto, CA, US): United States, 1999; Vol. 5872357, p 19.

(44) McDaniel, E. W.; Mason, E. A. Mobility and diffusion of ions in gases; John Wiley and Sons, Inc., New York: New York, 1973.

(45) Boens, N.; Qin, W.; Basarić, N.; Hofkens, J.; Ameloot, M.; Pouget, J.; Lefèvre, J.-P.; Valeur, B.; Gratton, E.; vandeVen, M.; Silva, N. D.; Engelborghs, Y.; Willaert, K.; Sillen, A.; Rumbles, G.; Phillips, D.; Visser, A. J. W. G.; van Hoek, A.; Lakowicz, J. R.; Malak, H.; Gryczynski, I.; Szabo, A. G.; Krajcarski, D. T.; Tamai, N.; Miura, A. Anal. Chem. 2007, 79, 2137.

(46) Frisch, M. J.; Trucks, G. W.; Schlegel, H. B.; Scuseria, G. E.; Robb, M. A.; Cheeseman, J. R.; Montgomery, J., J. A.; Vreven, T.; Kudin, K. N.; Burant, J. C.; Millam, J. M.; Iyengar, S. S.; Tomasi, J.; Barone, V.; Mennucci, B.; Cossi, M.; Scalmani, G.; Rega, N.; Petersson, G. A.; Nakatsuji, H.; Hada, M.; Ehara, M.; Toyota, K.; Fukuda, R.; Hasegawa, J.; Ishida, M.; Nakajima, T.; Honda, Y.; Kitao, O.; Nakai, H.; Klene, M.; Li, X.; Knox, J. E.; Hratchian, H. P.; Cross, J. B.; Bakken, V.; Adamo, C.; Jaramillo, J.; Gomperts, R.; Stratmann, R. E.; Yazyev, O.; Austin, A. J.; Cammi, R.; Pomelli, C.; Ochterski, J. W.; Ayala, P. Y.; Morokuma, K.; Voth, G. A.; Salvador, P.; Dannenberg, J. J.; Zakrzewski, V. G.; Dapprich, S.; Daniels, A. D.; Strain, M. C.; Farkas, O.; Malick, D. 
K.; Rabuck, A. D.; Raghavachari, K.; Foresman, J. B.; Ortiz, J. V.; Cui, Q.; Baboul, A. G.; Clifford, S.; Cioslowski, J.; Stefanov, B. B.; Liu, G.; Liashenko, A.; Piskorz, P.; Komaromi, I.; Martin, R. L.; Fox, D. J.; Keith, T.; Al-Laham, M. A.; Peng, C. Y.; Nanayakkara, A.; Challacombe, M.; Gill, P. M. W.; Johnson, B.; Chen, W.; Wong, M. W.; Gonzalez, C.; Pople, J. A. In Gaussian, Inc. Wallingford CT, 2004.

(47) Mesleh, M. F.; Hunter, J. M.; Shvartsburg, A. A.; Schatz, G. C.; Jarrold, M. F. J. Phys. Chem. 1996, 100, 16082.

(48) Shvartsburg, A. A.; Jarrold, M. F. Chem. Phys. Lett. 1996, 261, 86.

(49) Kim, H. I.; Kim, H.; Pang, E. S.; Ryu, E. K.; Beegle, L. W.; Loo, J. A.; Goddard, W. A.; Kanik, I. Anal. Chem. 2009, 81, 8289.

(50) Campuzano, I.; Bush, M. F.; Robinson, C. V.; Beaumont, C.; Richardson, K.; Kim, H.; Kim, H. I. Anal. Chem. 2011, 84, 1026.

(51) Singh, U. C.; Kollman, P. A. J. Comput. Chem. 1984, 5, 129.

(52) Besler, B. H.; Merz, K. M.; Kollman, P. A. J. Comput. Chem. 1990, 11, 431.

(53) Larriba, C.; Hogan, C. J., Jr. J Phys Chem A 2013, 117, 3887.

(54) Sengupta, A.; Singh, R. K.; Gavvala, K.; Koninti, R. K.; Mukherjee, A.; Hazra, P. J. Phys. Chem. B. 2014, 118, 1881.

(55) Radoszkowicz, L.; Huppert, D.; Nachliel, E.; Gutman, M. J. Phys. Chem. A. 2009, 114, 1017.

(56) Ross, S. K.; McDonald, G.; Marchant, S. Analyst. 2008, 133, 602.

(57) Sengupta, A.; Khade, R. V.; Hazra, P. J Photoch Photobio A 2011, 221, 105.

(58) Eiceman, G. A.; Krylov, E. V.; Krylova, N. S.; Nazarov, E. G.; Miller, R. A. Anal. Chem. 2004, 76, 4937.

(59) Fernández-Maestre, R.; Wu, C.; Hill, H. H. Rapid Commun. Mass Spectrom. 2012, 26, 2211.

(60) Dwivedi, P.; Wu, C.; Matz, L. M.; Clowers, B. H.; Siems, W. F.; Hill, H. H., Jr. Anal. Chem. 2006, 78, 8200. 


\section{CHAPTER 3.}

INSIGHTS FROM ION MOBILITY - MASS SPECTROMETRY, INFRARED SPECTROSCOPY, AND MOLECULAR DYNAMICS ON NICOTINAMIDE ADENINE DINUCLEOTIDE STRUCTURAL DYNAMICS: NAD ${ }^{+} V S$ NADH

This chapter was published in Physical Chemistry Chemical Physics and adapted with permission from all authors.

J. C. Molano-Arevalo, W. Gonzales, K. Jeanne Dit Fouque, J. Miksovska, P. Maître, F. Fernandez-Lima. Phys. Chem. Chem. Phys. 2018, 20, 7043 - 7052. 


\begin{abstract}
Nicotinamide adenine dinucleotide (NAD) is found in all living cells where the oxidized $\left(\mathrm{NAD}^{+}\right)$and reduced $(\mathrm{NADH})$ forms play important roles in many enzymatic reactions. However, little is known about $\mathrm{NAD}^{+}$and $\mathrm{NADH}$ conformational changes and kinetics as a function of the cell environment. In the present work, an analytical workflow is utilized to study $\mathrm{NAD}^{+}$and NADH dynamics as a function of the organic content in solution using fluorescence lifetime spectroscopy and in the gas-phase using trapped ion mobility spectrometry coupled to mass spectrometry (TIMS-MS) and infrared multiple photon dissociation (IRMPD) spectroscopy. NAD solution time decay studies showed a twocomponent distribution, assigned to changes from a "close" to "open" conformation with the increase of the organic content. NAD gas-phase studies using nESI-TIMS-MS displayed two ion mobility bands for $\mathrm{NAD}^{+}$protonated and sodiated species, while four and two ion mobility bands were observed for NADH protonated and sodiated species, respectively. Changes in the mobility profiles were observed for NADH as a function of the starting solution condition and the time after desolvation, while $\mathrm{NAD}^{+}$profiles showed no dependence. IRMPD spectroscopy of $\mathrm{NAD}^{+}$and NADH protonated species in the 8001800 and $3200-3700 \mathrm{~cm}^{-1}$ spectral regions showed common and signature bands between the NAD forms. Candidate structures were proposed for $\mathrm{NAD}^{+}$and $\mathrm{NADH}$ kinetically trapped intermediates of the protonated and sodiated species, based on their collision cross sections and IR profiles. Results showed that NAD species exist in an open, stack, and closed conformations and that the driving force for conformational dynamics is via hydrogen bonding of the $\mathrm{N}-\mathrm{H}-\mathrm{O}$ and $\mathrm{O}-\mathrm{H}-\mathrm{O}$ form with the ribose rings.
\end{abstract}




\section{INTRODUCTION}

Nicotinamide adenine dinucleotide (NAD) is a ubiquitous molecule found in all living cells. The structure of NAD consists of two nucleotides, ribose rings with adenine and nicotinamide joined together by a diphosphate bond. ${ }^{1}$ The oxidized $\left(\mathrm{NAD}^{+}\right)$and reduced (NADH) forms of NAD have important roles in cellular metabolism, functioning both as hydride-accepting and hydride-donating coenzymes in over 300 enzymatically catalyzed oxidation-reduction reactions, that control transcription and gene expression, DNA repair, regulation of energy metabolism, cell death, and aging. ${ }^{2-13}$ Besides serving as a multipurpose coenzyme, NAD is also used as a substrate of NAD-dependent ligases, NADdependent oxidoreductases, poly(ADP-ribose) polymerase (PARP) and the NADdependent deacetylases of the Sir2p family. $70,14-18$

Numerous human diseases are linked to fluctuations in the ratio between $\mathrm{NAD}^{+}$and $\mathrm{NADH}$ forms.${ }^{19} \mathrm{NAD}^{+}$is converted to NADH mostly in catabolic reactions including glycolysis and the tricarboxylic acid cycle. ${ }^{20}$ This delicate balance between the levels of $\mathrm{NAD}^{+}$and $\mathrm{NADH}$ forms plays an important role in regulating the intracellular redox state and is often considered as a readout of the metabolic state as it fluctuates in response to a change in metabolism. ${ }^{21-25}$ The fluorescence emission of NADH has been used extensively for the study of the redox status of tissues when the cofactor is bound to enzymes, ${ }^{26-30}$ while the identification of free and bound NADH is challenging because the fluorescence decay times are on the sub-nanosecond timescale. ${ }^{31}$ Several studies have been focused on the theoretical structural interrogation of both $\mathrm{NAD}^{+}$and $\mathrm{NADH}$. $\mathrm{Ab}$ initio calculations were used to evaluate the conformational preferences of the nicotinamide ring while $\mathrm{NAD}^{+}$and 
NADH were bound to dependent dehydrogenases. ${ }^{32}$ It was found that the redox potential of the cofactor is a function of the ribose orientation, were the glycosidic $\mathrm{C}-\mathrm{O}$ bond of $\mathrm{NAD}^{+}$is near the plane of the nicotinamide ring, while the glycosidic C-O bond of NADH is nearly perpendicular to the dihydronicotinamide ring. The parametrization of empirical force fields for the modelling of NAD was performed following the methodology used in the development of CHARMM22 all-hydrogens parameters for proteins, nucleic acids, and lipids. ${ }^{33}$ Molecular dynamic simulations of $\mathrm{NAD}^{+}$in the presence of different solvents showed the presence of folded and extended conformations. ${ }^{34}$

Recently, trapped ion mobility spectrometry (TIMS) was used successfully in combination with fluorescence time decay studies and molecular dynamics in order to characterize the conformational populations of flavin adenine dinucleotide in solution and in the gasphase. ${ }^{35}$ The combination of these techniques has proven to be a versatile and powerful analytical workflow in the study of intermediate and equilibrium structures of biomolecules. ${ }^{35-38}$ More recent development of analytical instrumentation integrating both ion mobility separation and optical spectroscopy, either in the infrared, ${ }^{39,40}$ or UV-visible ${ }^{41,42}$ regions, has been shown to be effective for providing structural information on massselected ions. Infrared free electron lasers (IR FEL) and optical parametric oscillator/amplifier (OPO/A) benchtop lasers provide access to a wide frequency range, allowing to record vibrational spectra in the mid-infrared and in the $\mathrm{X}-\mathrm{H}(\mathrm{X}=\mathrm{C}, \mathrm{N}, \mathrm{O})$ stretching regions, respectively. This so-called action spectroscopy has been particularly successful for distinguishing isomers ${ }^{43,44}$ and unravelling the hydrogen bonding association to peptide structuration. ${ }^{45,46}$ 
In the present work, $\mathrm{NAD}^{+}$and $\mathrm{NADH}$ forms were studied as a function of the solution organic content using fluorescence lifetime spectroscopy, TIMS-MS with collision induced activation (CIA), IRMPD spectroscopy and molecular dynamics. Candidate structures were proposed for the kinetically trapped intermediates based on ion-neutral collision cross section $\left(\mathrm{CCS}_{\mathrm{N} 2}\right)$, IRMPD spectroscopy, and molecular dynamics. In particular, this study focuses on describing the differences in the intramolecular interactions of $\mathrm{NAD}^{+}$and $\mathrm{NADH}$.

\section{MATERIALS AND METHODS}

Materials and reagents

$\beta$-Nicotinamide adenine dinucleotide disodium salt hydrate (EC number 210-123-3) powder was purchased from Sigma-Aldrich (St. Louis, MO). All solvents and ammonium acetate salts were analytical grade or better and purchased from Fisher Scientific (Pittsburg, PA). A stock solution was prepared in $10 \mathrm{mM}$ ammonium acetate $(\mathrm{pH} \mathrm{7.0)}$ and aliquots were diluted to a final concentration of $10 \mu \mathrm{M}$ in 100:00 to 50:50 (v/v) watermethanol/ethanol solutions in stepwise increments of $10 \%$ organic content. A Tuning Mix calibration standard (TuneMix, G24221A) was purchased from Agilent Technologies (Santa Clara, CA). Details on the Tuning Mix structures (e.g., $m / z 322 K_{0}=1.376 \mathrm{~cm}^{2} \mathrm{~V}^{-1}$ $\mathrm{s}^{-1}$ and $m / z 622 K_{0}=1.013 \mathrm{~cm}^{2} \mathrm{~V}^{-1} \mathrm{~s}^{-1}$ ) can be found elsewhere. ${ }^{47,48}$

\section{NanoESI-CIA-TIMS-MS Analysis}

Ion mobility experiments were performed on a custom built nanoESI-TIMS coupled to a maXis Impact Q-ToF mass spectrometer (Bruker Daltonics Inc., MA). A $10 \mu \mathrm{L}$ aliquot of the sample solution was loaded in the pulled-tip capillary and sprayed at 600-1200 V. 
Details regarding the TIMS operation and specifics compared to traditional IMS can be found elsewhere (the TIMS cell schematics can be found in Figure S1). ${ }^{48-50}$ Briefly, TIMS ion mobility separation is based on holding the ions stationary using an electric field against a moving gas. The separation in a TIMS device can be described in the center of mass frame using the same principles as in a conventional IMS drift tube. ${ }^{51}$ Since ion mobility separation is related to the number of ion-neutral collisions (or drift time in traditional drift tube cells), the ion mobility separation in a TIMS device depends on the bath gas drift velocity, ion confinement and ion elution parameters. The mobility, $K$, of an ion in a TIMS cell is described by:

$K=\frac{v_{g}}{E}=\frac{A}{\left(V_{\text {elution }}-V_{\text {out }}\right)}$

where $v_{g}, E, V_{\text {elution }}$ and $V_{\text {out }}$ are the velocity of the gas, applied electric field, elution and last electrode voltages, respectively. The constant $A$ can be determined using calibration standards of known mobilities. In TIMS operation, multiple isomers/conformers are trapped simultaneously at different $E$ values resulting from a voltage gradient applied across the IMS tunnel region. After thermalization, isomers/conformers are eluted by decreasing the electric field in stepwise decrements (referred to as the "ramp"). Each isomer/conformer eluting from the TIMS cell can be described by a characteristic voltage ( $\left.V_{\text {elution }}\right)$. In a TIMS device, the total analysis time $\left(\mathrm{t}_{\text {Total }}\right)$ can be described as:

$t_{T}=t_{\text {trap }}+\left(\frac{V_{\text {elution }}}{V_{\text {ramp }}}\right) t_{\text {ramp }}+$ tof $=t_{o}+\left(\frac{V_{\text {elution }}}{V_{\text {ramp }}}\right) t_{\text {ramp }}(2)$

where, $t_{\text {trap }}$ is the thermalization/trapping time, tof is the time after the ion mobility separation, and $V_{\text {ramp }}$ and $t_{\text {ramp }}$ are the voltage range and time required to vary the electric 
field, respectively. ${ }^{37,38}$ The elution voltage can be experimentally determined by varying the ramp time for a constant ramp voltage range.

The TIMS funnel is controlled using in-house software, written in National Instruments Lab VIEW, and synchronized with the maXis Impact Q-ToF acquisition program. ${ }^{49}, 50$ TIMS separation was performed using nitrogen as a bath gas at $c a .300 \mathrm{~K}$, and the gas flow velocity was controlled by the pressure difference between entrance funnel $P_{1}=2.6 \mathrm{mbar}$, and the exit funnel $P_{2}=1.0 \mathrm{mbar} . P_{1}$ and $P_{2}$ values were held constant for all experiments. The same RF ( $880 \mathrm{kHz}$ and $200 \mathrm{Vpp}$ ) was applied to all electrodes including the entrance funnel, the ion mobility separating section, and the exit funnel. The TIMS cell was operated using a fill/trap/ramp/wait sequence of 10/10/100-500/50 ms. ${ }^{49,50}$

Mobility values $(K)$ were correlated with $\operatorname{CCS}(\Omega)$ using the equation:

$\Omega=\frac{(18 \pi)^{1 / 2}}{16} \frac{z}{\left(k_{B} T\right)^{1 / 2}}\left(\frac{1}{m_{I}}+\frac{1}{m_{b}}\right)^{1 / 2} \frac{1}{K} \frac{760}{P} \frac{T}{273.15} \frac{1}{N^{*}}$

where $z$ is the charge of the ion, $k_{B}$ is the Boltzmann constant, $N^{*}$ is the number density of the bath gas and $m_{I}$ and $m_{b}$ refer to the masses of the ion and bath gas, respectively. ${ }^{51}$ All resolving power $(\mathrm{R})$ values reported herein were determined from Gaussian peak fits of the features in the TIMS distributions ( $\mathrm{R}=\Omega / \Delta \Omega$ ) using OriginPro (version 9.3.226). The FWHM of the mobility band was used to calculate the $\Delta \Omega$.

Collision induced activation (CIA) experiments were performed to assess the effect of the activation energy on the conformational space of NAD. Soft activation energy conditions were implemented to study the memory effect from the starting solution (capillary $\left(V_{c a p}\right)$ : $50 \mathrm{~V}$; deflector start $\left(V_{d e f}\right): 60 \mathrm{~V}$; entrance funnel $\left.\left(V_{f u n}\right): 0 \mathrm{~V}\right)$. For CIA experiments, the injection energy was incremented by $10 \mathrm{~V}$ steps until $V_{\text {cap }}: 190 \mathrm{~V}, V_{\text {def: }}: 200 \mathrm{~V}$ and $V_{\text {fun }}: 150$ 
V. A total of 500 accumulations and 10 frames were acquired per ramp time (e.g., $t_{\text {ramp }}=$ 100-500 ms).

Photo-physical characterization of NADH in solution

All fluorescence measurements were conducted using a PC1-ChronosFD custom instrument (ISS, Champaign Illinois). NAD was used without further purification and diluted from powder kept at $-20{ }^{\circ} \mathrm{C}$ into $10 \mathrm{mM}$ ammonium acetate buffer at $\mathrm{pH} 8$ at 100 $\mu \mathrm{M}$ concentration, and ethanol or methanol was added to the desired $\mathrm{v} / \mathrm{v}$ ratio. All measurements were conducted at a room temperature of $\sim 18{ }^{\circ} \mathrm{C}$. Steady-state emission spectra were obtained by exciting the sample with $350 \pm 4 \mathrm{~nm}$ light along the $2 \mathrm{~mm}$ path of a $2 \times 10 \mathrm{~mm}$ quartz cuvette, and the emission was collected through a vertical polarizer with an emission bandwidth of $\pm 4 \mathrm{~nm}$. Fluorescence and anisotropy decay experiments were performed in the frequency domain mode. NADH solutions were excited using a 370 $\mathrm{nm}$ intensity modulated laser diode and fluorescent emission was collected using a $400 \mathrm{~nm}$ long pass filter (Andover, Salem, NH). A solution of POPOP (1,4-bis(5-phenyloxazol-2yl)benzene) in ethanol was used as a lifetime reference. Polarizers were set at the magic angle configuration for the lifetime measurements. ${ }^{52}$ Modulation-phase data were analyzed using GlobalsWE software (Laboratory of Fluorescence Dynamics, Irvine, CA) ${ }^{53}$ and the $\chi^{2}$ parameter was used as criterion for goodness of fit. ${ }^{54}$

\section{ESI-FT-ICR MS-IRMPD Analysis}

Infrared action spectroscopy experiments were carried out employing a $7 \mathrm{~T}$ Fourier transform ion cyclotron resonance (FT-ICR) mass spectrometer (Apex Qe, Bruker) coupled with tunable infrared lasers at the CLIO facility (Orsay, France). A detailed layout of this

experimental apparatus is described elsewhere. ${ }^{55}$ Mass-selected ions were accumulated in 
an argon pressurized linear hexapole ion trap. Ions were then pulse extracted and stored in the ICR cell where they were irradiated with infrared light. Infrared action spectroscopy was carried out by monitoring the intensities of precursor $\left(I_{\text {precursor }}\right)$ and resulting fragment ions $\left(I_{\text {fragment }}\right)$ as a function of the laser wavenumber. The infrared action spectra were obtained by plotting the photo-dissociation efficiencies, defined as $\ln (1+$ $\Sigma I_{\text {fragment }}\left(I_{\text {precursor }}\right)$, as a function of the laser wavenumber. Infrared action spectra of $\mathrm{NAD}^{+}$ and NADH were recorded in the $3200-3700 \mathrm{~cm}^{-1}$ spectral range using an optical parametric oscillator/amplifier (OPO/A from Laser Vision, Bellevue, WA) benchtop laser. ${ }^{56}$ The irradiation time was $1 \mathrm{~s}$. In order to enhance the infrared induced fragmentation efficiency, an auxiliary broadband $\mathrm{CO}_{2}$ laser (BFI Optilas, Evry, France) was used. ${ }^{57}$ The $\mathrm{CO}_{2}$ pulse length was $500 \mu$ s for both $\mathrm{NAD}^{+}$and NADH. Infrared spectroscopy in the $800-1800 \mathrm{~cm}^{-1}$ spectral range was performed using the free electron laser (FEL, from CLIO, Orsay, France). ${ }^{58}$ The irradiation time was set to $700 \mathrm{~ms}$ for both $\mathrm{NAD}^{+}$and NADH to record vibrational spectra in the mid-infrared.

Theoretical Calculations

A pool of candidate structures was proposed for the IMS bands observed in the nESITIMS-MS experiments. Briefly, consecutive molecular dynamics simulations were used to reproduce the experimental conditions (e.g. "TIMS" thermostat) in order to generate the initial pool of structures. This approach is equivalent to that previously described by Fernández-Lima and co-workers. ${ }^{59}$ Molecular dynamics simulations of annealing and geometry optimization cycles were carried out in a NVT thermostat using AMBER03 ${ }^{60}$ force field in YASARA software.$^{61}$ Final structures were optimized at the DFT/B3LYP/631G* level using Jaguar (Schrödinger, LLC, Cambridge, MA) ${ }^{62}$ Vibrational frequencies 
were calculated and scaled by 0.961 , according to the Computational Chemistry Comparison and Benchmark DataBase NIST. ${ }^{63}$ Zero-point energy corrections were applied to the relative stability analysis between the structures. Theoretical ion-neutral collision cross sections were calculated using MOBCAL version for helium, ${ }^{64,65}$ nitrogen, ${ }^{66}$ and the software package iMos ${ }^{67}{ }^{68}$ Partial atomic charges were calculated using the Merz-SinghKollman scheme constrained to the molecular dipole moment. ${ }^{69,70}$

\section{RESULTS AND DISCUSSION}

nESI-TIMS-MS analysis of NAD showed protonated and sodiated molecular ions (Figure 3.1). The most abundant $[\mathrm{M}+\mathrm{H}]^{+}$and $[\mathrm{M}+\mathrm{Na}]^{+}$ions correspond to the reduced form, NADH ( $\left.\mathrm{M}=\mathrm{C}_{21} \mathrm{H}_{29} \mathrm{~N}_{7} \mathrm{O}_{14} \mathrm{P}_{2}\right)$, while similar molecular ions were observed for the oxidized form, $\mathrm{NAD}^{+}\left(\mathrm{M}=\mathrm{C}_{21} \mathrm{H}_{27} \mathrm{~N}_{7} \mathrm{O}_{14} \mathrm{P}_{2}\right)$.

nESI-TIMS-MS as a function of the organic content in solution (e.g. 0-50 $\mathrm{mM} \mathrm{NH}_{4} \mathrm{Ac}, 0$ $50 \% \mathrm{MeOH}$ or EtOH) and activation energy (CIA-TIMS-MS) showed multiple IMS bands for the protonated and sodiated species of $\mathrm{NAD}^{+}$and NADH (labels A-J in Figure 3.2). Inspection of the ion mobility profile for $\left[\mathrm{M}_{\mathrm{NADH}}+\mathrm{H}\right]^{+}$showed four mobility bands (labels A-D) for all starting solutions. Moreover, the relative abundance of B was greater when the solution contains $\mathrm{NH}_{4} \mathrm{Ac}$ or $\mathrm{EtOH}$, while $\mathrm{C}$ was more abundant when $\mathrm{MeOH}$ was added to the starting solution. For $\left[\mathrm{M}_{\mathrm{NADH}}+\mathrm{Na}\right]^{+}($labels $\mathrm{E}$ and $\mathrm{F})$, the intensity of $\mathrm{E}$ was slightly greater than $\mathrm{F}$ in the $\mathrm{NH}_{4} \mathrm{Ac}$ solution, considerably greater when $\mathrm{MeOH}$ was added, and smaller than $\mathrm{F}$ when EtOH was added. These changes in the relative abundances of the mobility bands suggests that, at the molecular level in solution, the interaction with the organic molecules drives the equilibria between the multiple conformations. Major 
differences in the relative abundances of the ion mobility profiles for $\left[\mathrm{M}_{\mathrm{NAD}^{+}}+\mathrm{H}\right]^{+}$(labels G and $\mathrm{H}$ ), and $\left[\mathrm{M}_{\mathrm{NAD}+}+\mathrm{Na}\right]^{+}$(labels I and $\mathrm{J}$ ) were not observed as a function of the starting solution conditions (Figure 3.2) and the trapping time (e.g.; 100-500 ms, data not shown). This suggests that the species formed during the nanoESI process are stable in the TIMSMS experiments time scale, and that the solvation effects of the organic molecules are not enough to favor conformational interconversion of the oxidized NAD. Moreover, no changes in the $\mathrm{CCS}_{\mathrm{N} 2}$ values were observed as a function of the organic content in the starting solutions, which suggests that the observed ion mobility bands correspond to different conformations of NAD and not to organic solvent clustering with the molecular ions in the gas-phase.

Further sampling of the conformational space of NAD in the gas phase was performed via collision induced activation (CIA) prior to the ion mobility separation (Figure 3.2, red panel). While the same number of mobility bands were observed as a function of the CIA condition, some changes in the relative abundances of the mobility bands were observed; we interpret this variation as the interconversion between the most energetically favorable conformational populations. Moreover, population interconversion between NADH mobility bands was observed as a function of the trapping time (e.g., 100-500 ms) and the organic content (e.g., $\mathrm{MeOH}$ and $\mathrm{EtOH}$ ) for the protonated and sodiated species (Figure 3.3). Inspection of the mobility profiles showed a decrease (red pattern area under the curve) of $\mathrm{B}$ for $\left[\mathrm{M}_{\mathrm{NADH}}+\mathrm{H}\right]^{+}$, favoring the increase (blue pattern area under the curve) in the abundance of C. Similarly, inspection of the profiles for $\left[\mathrm{M}_{\mathrm{NADH}}+\mathrm{Na}\right]^{+}$showed that the relative abundance of $\mathrm{E}$ increased while the abundance of $\mathrm{F}$ mobility band decreased. These results suggested that kinetically trapped intermediates can interconvert into other local 
free energy minima after thermalization in the TIMS cell. ${ }^{71}$ The effect of the nature of organic content on the conformational space was observed by changes in the starting relative abundance of the mobility bands, for example, $\mathrm{B}$ and $\mathrm{C}$ mobility bands for [ $\mathrm{M}_{\mathrm{NADH}}$ $+\mathrm{H}]^{+}$and $\mathrm{E}$ and $\mathrm{F}$ mobility bands for $\left[\mathrm{M}_{\mathrm{NADH}}+\mathrm{Na}\right]^{+}$showed different relative abundances with the starting solution organic content ( $\mathrm{MeOH} v s \mathrm{EtOH})$.

The heterogeneity of NAD in solution was characterized by measuring the NAD lifetime as a function of the organic content (e.g., 10-70\% methanol and ethanol) using frequency domain florescence spectroscopy (Figure 3.4). The data were analyzed using a double exponential decay model and the results are summarized in Table S3.1. Two different components that can be attributed to different conformational groups were resolved: a fast decaying component of $0.3 \mathrm{~ns}$, attributed to intermediates with a "closed" conformation; and a slow component of $0.94 \mathrm{~ns}$, attributed to intermediates belonging to both a "stack" and "open" conformations. ${ }^{31}$ Inspection of figure 3.4 shows that a change in the solution dielectric constant with the organic content, alters the fraction of the "stack" and "open" conformation. The analysis in methanol and ethanol showed the fast and slow decay times with a faster change in the fast/slow ratio for ethanol when compared to methanol. These observations are in good agreement with the trends observed during the TIMS-MS analysis (i.e., kinetic trapped intermediates, Figure 3.3) and support the hypothesis that "memory effects" of the starting solution can be retained in the mobility profiles using "soft" ion transmission settings in the TIMS-MS experiments.

Complementary studies of $\mathrm{NAD}^{+}$and NADH using IRMPD spectroscopy permitted the interrogation of the chemical local environment of the gas-phase ions (Figure 3.5). Comparison of IR spectra of $\mathrm{NAD}^{+}$and NADH protonated species showed the presence of 
common as well as signature bands. A tentative assignment of the observed infrared bands and the atoms involved in the intramolecular interactions of the protonated species of $\mathrm{NAD}^{+}$and $\mathrm{NADH}$ are proposed in Tables S3.3 and S3.4. In the high energy range (e.g., $3300-3700 \mathrm{~cm}^{-1}$ ), the bands observed at $\sim 3660 \mathrm{~cm}^{-1}$ were present for both NADH and $\mathrm{NAD}^{+}$forms and were assigned to free phosphate $\mathrm{OH}$ stretches (Figure 3.5$) .{ }^{72}$ The bands observed near $\sim 3560 \mathrm{~cm}^{-1}$ are typical signatures of free $\mathrm{NH}_{2}$ asymmetric stretches. ${ }^{73}$ Two other common bands between $\mathrm{NAD}^{+}$and $\mathrm{NADH}$ were observed at $\sim 3430$ and $\sim 3540 \mathrm{~cm}^{-1}$, and could be tentatively assigned to $\mathrm{N}-\mathrm{H}-\mathrm{O}$ and $\mathrm{O}-\mathrm{H}-\mathrm{O}$ stretch interactions, respectively. ${ }^{74,75}$

A clear evidence for changes in the hydrogen bonding network between the $\mathrm{NAD}^{+}$and NADH could be observed in the OPO spectral range. While no band was observed in the case of NADH, a band at $\sim 3480 \mathrm{~cm}^{-1}$ was observed for NAD ${ }^{+}$. The $\sim 3480 \mathrm{~cm}^{-1}$ band could be assigned to red-shifted alcohol $\mathrm{OH}$ stretches. This assignment was further supported by the differences in the infrared spectra of $\mathrm{NAD}^{+}$and NADH in the $800-1800 \mathrm{~cm}^{-1}$ spectral range. In fact, the $\mathrm{P}=\mathrm{O}$ stretch band was red-shifted $\left(\sim 1290 \mathrm{~cm}^{-1}\right)$ for $\mathrm{NAD}^{+}$, compared to $\mathrm{NADH}$ for where the corresponding band $\left(1335 \mathrm{~cm}^{-1}\right)$ is typical of free $\mathrm{P}=\mathrm{O}$ stretch and is indicative of the presence of an $\mathrm{O}-\mathrm{H}-\mathrm{O}$ stretch interaction (Figure 3.5). In addition, IRMPD spectra of NADH exhibited P-OH bend and stretch bands in the $900-1000 \mathrm{~cm}^{-1}$ spectral range, while these bands were observed with very low intensity in the case of $\mathrm{NAD}^{+}$. Moreover, IRMPD spectra of $\mathrm{NAD}^{+}$showed two signature bands at 1110 and 1255 $\mathrm{cm}^{-1}$, not present in the case of $\mathrm{NADH}$, corresponding to $\mathrm{PO}^{-}$symmetric and asymmetric stretches, respectively. That is, the P-OH stretch and P-OH bend bands in the IRMPD spectra of NADH suggest a non-zwitterionic form, while characteristic bands of PO2- 
asymmetric and symmetric stretches in the case of $\mathrm{NAD}^{+}$suggests that at least one of the phosphate groups is not protonated. In addition, comparison between IRMPD and FTIR spectroscopy of $\mathrm{NAD}^{+}$in solution at low $\mathrm{pH}$ showed similar profiles in the $900-1300 \mathrm{~cm}^{-1}$ spectral range. ${ }^{76}$ In fact, Nadolny and co-workers confirmed that the protonation of NAD ${ }^{+}$ is located on the adenine residue (labeled $\mathrm{N}^{9}$ in Figure S3.9) and that no additional proton is bound to phosphate group with decreasing $\mathrm{pH}$.

Further interpretation of the TIMS-MS and IRMPD spectra was assisted by the theoretical $\mathrm{CCS}_{\mathrm{N} 2}$ and IR profiles from candidate structures obtained from molecular dynamics (Figure 3.5 and Tables S3.3 and S3.4). The selection of candidate structures was performed by comparing the three lowest energy candidates per IMS band observed $\left(\mathrm{CCS}_{\mathrm{N} 2}\right.$ within $5 \%$ error) and their calculated IR spectra. Candidate structures of the sodiated forms of NAD can be found in Figure S3.12 and Table S3.2; moreover, since IRMPD spectra were not collected for the sodiated form, data is limited to the CCS and relative energy. The candidate structures of the protonated NAD species can be grouped in three main conformational families: "close" (structures A and G in Figures S3.2 and S3.6), "stack" (peaks B and C in Figures S3.3 and S3.4), and "open" (peaks D and H in Figures S3.5 and S3.7). Note that the atoms in the structures of $\mathrm{NAD}^{+}$and $\mathrm{NADH}$ are numbered to facilitate the visualization of the intramolecular interactions (Figure S3.9). Since the IRMPD spectra contain information from all conformers, candidate structure assignment assumed that for each mobility band, the proposed candidate structure per IMS band can match most but not necessary all the IRMPD bands. Further inspection of the candidate structures revealed more details on the intramolecular interactions that stabilize the kinetically trapped intermediates species of $\mathrm{NADH}$ and $\mathrm{NAD}^{+}$. For example, the theoretical calculations 
indicated intramolecular interactions involving an $\mathrm{N}^{2}-\mathrm{H}-\mathrm{O}^{11}$ and $\mathrm{O}^{5}-\mathrm{H}-\mathrm{O}^{11}$ for the two common bands observed at $\sim 3430$ and $\sim 3540 \mathrm{~cm}^{-1}$ (Figure S3.10 and Tables S3.3-S3.6). Furthermore, the characteristic band of $\mathrm{NAD}^{+}$observed near $\sim 3480 \mathrm{~cm}^{-1}$ is defined by an $\mathrm{O}^{3}-\mathrm{H}-\mathrm{O}^{6}$ intramolecular interaction. Theoretical calculations of the candidate structures (Figures S3.2-S3.7) also showed common as well as signature intramolecular interactions in both NADH and $\mathrm{NAD}^{+}$(Figure S3.10 and Tables S3.5 and S3.6). For example, the $\mathrm{O}^{6}$ $\mathrm{H}-\mathrm{O}^{10}, \mathrm{O}^{6}-\mathrm{H}-\mathrm{O}^{11}, \mathrm{O}^{6}-\mathrm{H}-\mathrm{N}^{9}, \mathrm{~N}^{8}-\mathrm{H}-\mathrm{O}^{12}$ and $\mathrm{O}^{4}-\mathrm{H}-\mathrm{O}^{6}$ intramolecular interactions appeared specific to the $\mathrm{NADH}$, while the $\mathrm{N}^{2}-\mathrm{H}-\mathrm{O}^{5}, \mathrm{O}^{5}-\mathrm{H}_{-} \mathrm{N}^{7}, \mathrm{O}^{6}-\mathrm{H}_{-} \mathrm{N}^{7}, \mathrm{~N}^{2}-\mathrm{H}-\mathrm{O}^{6}$ and $\mathrm{O}^{3}-\mathrm{H}-\mathrm{O}^{6}$ intramolecular interactions are involved in the $\mathrm{NAD}^{+}$form. That is, specific intramolecular interactions define the three main conformational families (Figure S3.11). For example, the $\mathrm{O}^{6}-\mathrm{H}-\mathrm{O}^{10}$ and $\mathrm{N}^{2}-\mathrm{H}-\mathrm{O}^{6}$ intramolecular interactions appeared specific to the "closed" conformation for $\mathrm{NADH}$ and $\mathrm{NAD}^{+}$, respectively. The $\mathrm{O}^{5}-\mathrm{H}-\mathrm{N}^{7}$ intramolecular interactions is characteristic to $\mathrm{NAD}^{+}$"open" conformations and the $\mathrm{N}^{8}-\mathrm{H}-\mathrm{O}^{12}$ intramolecular interactions is characteristic to NADH "stack" conformation. Common intramolecular interactions to the three conformational families are $\mathrm{O}^{1}-\mathrm{H}-\mathrm{N}^{8}, \mathrm{O}^{5}-\mathrm{H}-\mathrm{O}^{11} \mathrm{O}^{5}-\mathrm{H}-\mathrm{N}^{9}$, and $\mathrm{N}^{2}-$ $\mathrm{H}-\mathrm{O}^{11}$ interactions. While this information mostly reflects the findings made in the gasphase (e.g., TIMS-MS and IRMPD experiments), it can be extrapolated to better understand the possible mechanism that drive the conformational changes in solution, especially when memory effects of the solution conditions and similar trends with fluorescence lifetime spectroscopy and IRMPD were observed. 


\section{CONCLUSIONS}

This work highlights an analytical workflow for complementary solution and gas-phase studies of biomolecules that utilizes fluorescence lifetime spectroscopy, trapped ion mobility spectrometry coupled to mass spectrometry (TIMS-MS), infrared multiple photon dissociation spectroscopy (IRMPD) and molecular dynamics. The high resolving power of TIMS-MS permitted the separation of multiple IMS band and retain the solvent "memory" as shown with the variation of the starting organic content. IRMPD and theoretical calculations permitted the assignment of intramolecular interactions and highlighted the main differences between the oxidized and reduced NAD forms. IRMPD spectra of NADH suggest a non-zwitterionic form, while characteristic bands of $\mathrm{PO}^{-}$symmetric and asymmetric stretches in the case of $\mathrm{NAD}^{+}$suggests that at least one the phosphate groups is not protonated. For the first time, the intramolecular interactions that stabilize the conformational space of $\mathrm{NAD}^{+}$and $\mathrm{NADH}$ as well as the specific intramolecular interactions involved in the three main conformational families are described. The results obtained from the study of NAD in both solution and in the gas-phase, and the conformational exploration using molecular dynamics show that NAD species can exist in an "open", "stack", and "closed" conformations, and that the driving force for the structural stability of each group and their conformational dynamics is via hydrogen bonding. This study also highlights the need to further integrate TIMS-MS and IRMPD measurements in a single experiment to better differentiate the motifs that stabilize different mobility bands as suggested by others. ${ }^{39,40}$ 


\section{ASSOCIATED CONTENT}

TIMS instrument scheme, lowest energy candidate structures proposed for NADH and $\mathrm{NAD}^{+}$IMS bands, theoretical IR spectra of $\mathrm{NADH}$ and $\mathrm{NAD}^{+}$, Labelling of the atoms present in the structure of NADH and $\mathrm{NAD}^{+}$, intramolecular interactions of NADH and $\mathrm{NAD}^{+}$, Fluorescence decay parameters of $\mathrm{NADH}$ as a function of $\mathrm{EtOH}$ or $\mathrm{MeOH}$ content, experimental and theoretical CCS and experimental vibrational frequencies for the

protonated $\mathrm{NAD}^{+}$and NADH forms, experimental and theoretical CCS for the sodiated $\mathrm{NAD}^{+}$and $\mathrm{NADH}$ forms, and theoretical intramolecular interactions of $\mathrm{NADH}$ and $\mathrm{NAD}^{+}$.

\section{ACKNOWLEDGMENTS}

The authors wish to acknowledge Dr. Mark E. Ridgeway and Dr. Melvin A. Park from Bruker Daltonics, Inc. during the development and implementation of TIMS-MS experiments. The authors want to thank MSc. Vincent Steinmetz for the support during the collection and processing of the IRMPD data in the CLIO/CNRS facility. 
Figure 3.1 Structures of the oxidized $\left(\mathrm{M}=\mathrm{C}_{21} \mathrm{H}_{27} \mathrm{~N}_{7} \mathrm{O}_{14} \mathrm{P}_{2}\right)$ and reduced $(\mathrm{M}=$ $\left.\mathrm{C}_{21} \mathrm{H}_{29} \mathrm{~N}_{7} \mathrm{O}_{14} \mathrm{P}_{2}\right)$ NAD forms. Typical MS spectra of protonated and sodiated molecular ions of both NAD species are shown.
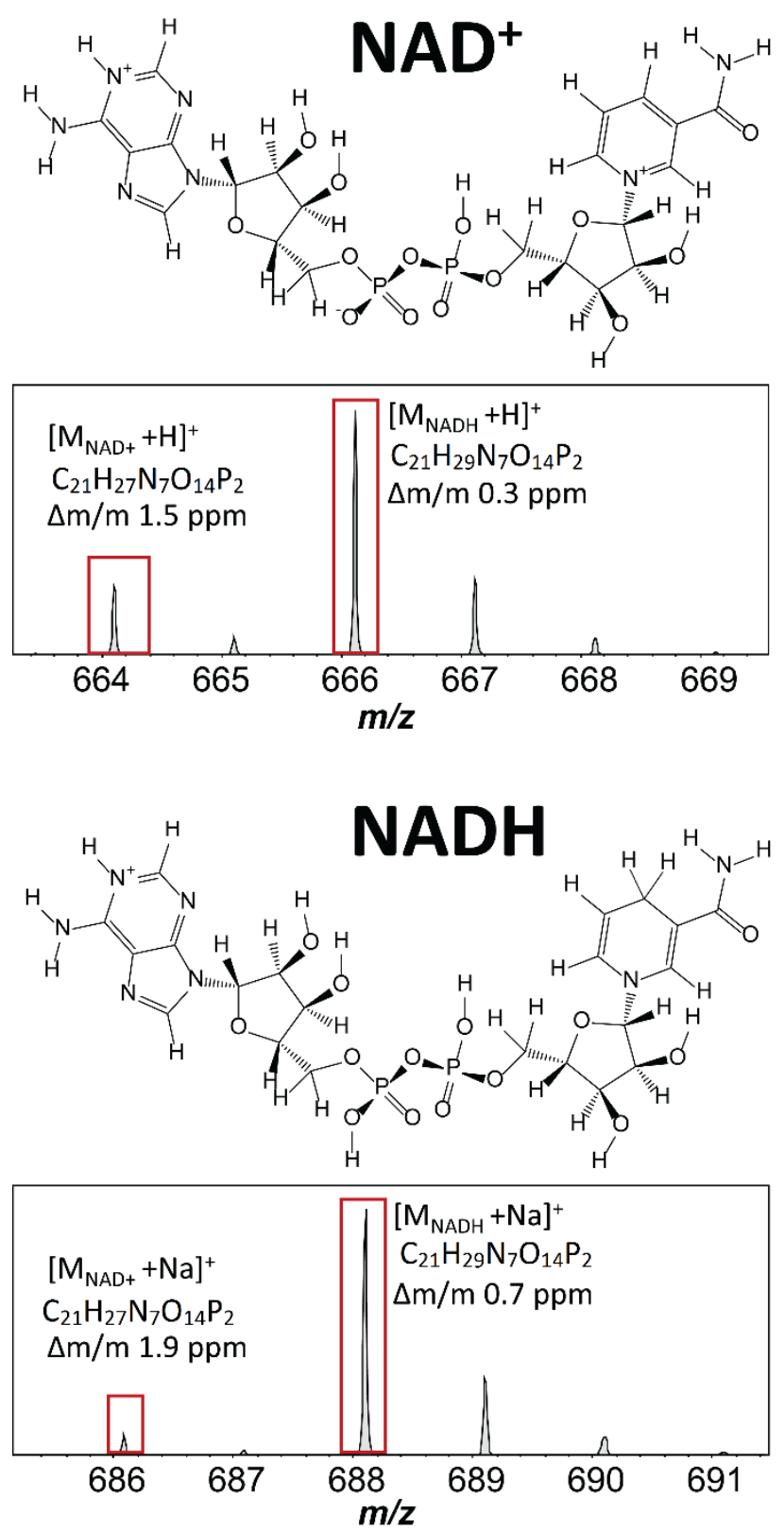
Figure 3.2 Ion mobility profiles of NAD $\left(\mathrm{t}_{\text {trap }}=500 \mathrm{~ms}\right)$ as a function of the organic content in the starting solution (blue panel) and the collision induced activation energy (red panel). Labels A-D are assigned to the IMS bands of the NADH $[\mathrm{M}+\mathrm{H}]^{+}$species; E-F to $\mathrm{NADH}[\mathrm{M}+\mathrm{Na}]^{+}$species; G-H to $\mathrm{NAD}^{+}[\mathrm{M}+\mathrm{H}]^{+}$species; and I-J to NAD ${ }^{+}[\mathrm{M}+\mathrm{Na}]^{+}$ species.
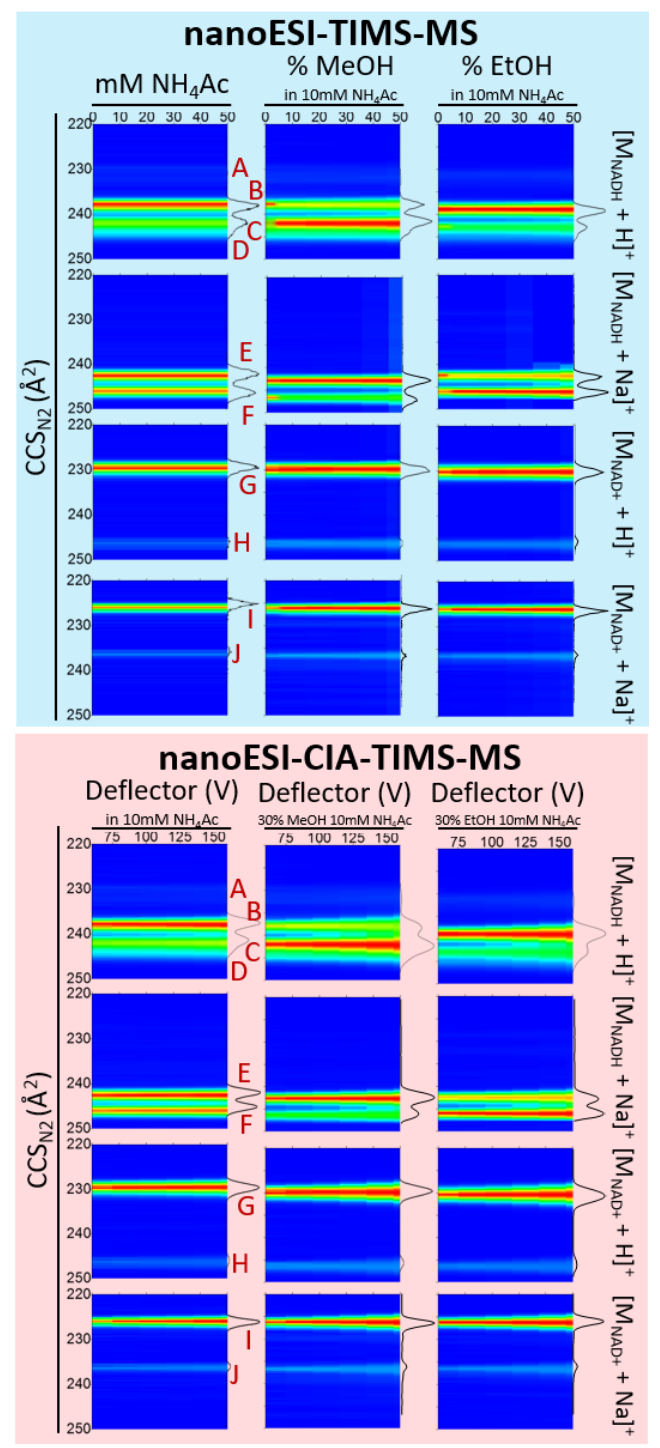
Figure 3.3 Typical IMS and interconversion plots as a function of the trapping time and starting solvent conditions (70:30 $\mathrm{H}_{2} \mathrm{O}: \mathrm{MeOH}$ and $\left.\mathrm{H}_{2} \mathrm{O}: \mathrm{EtOH}\right)$ for $\mathrm{NADH}[\mathrm{M}+\mathrm{H}]^{+}$and $[\mathrm{M}+\mathrm{Na}]^{+}$species.

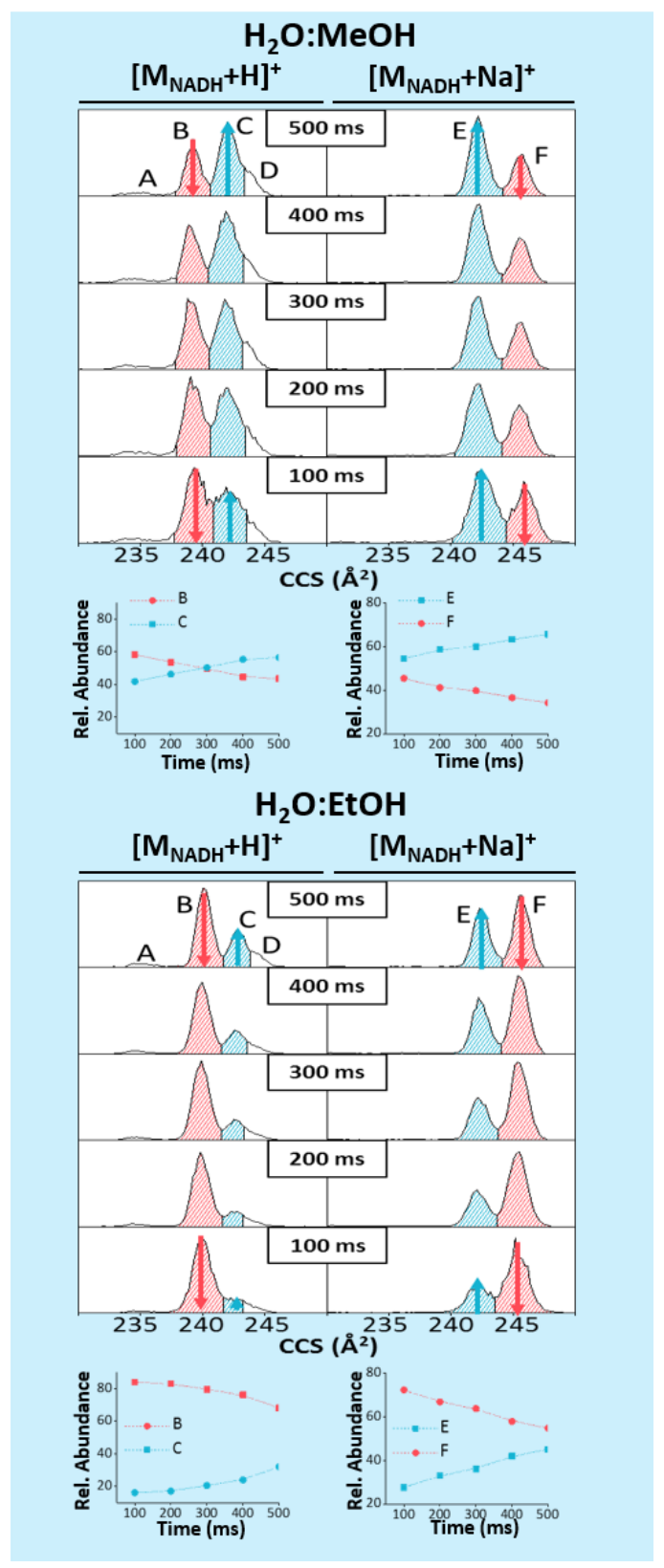


Figure 3.4 Emission spectra of NAD as a function of the organic content. In the inset, changes in the pre-exponential factor $(\alpha)$ is shown as a function organic content for the fast $\left(\tau_{0}\right)$ and slow $\left(\tau_{1}\right)$ components.

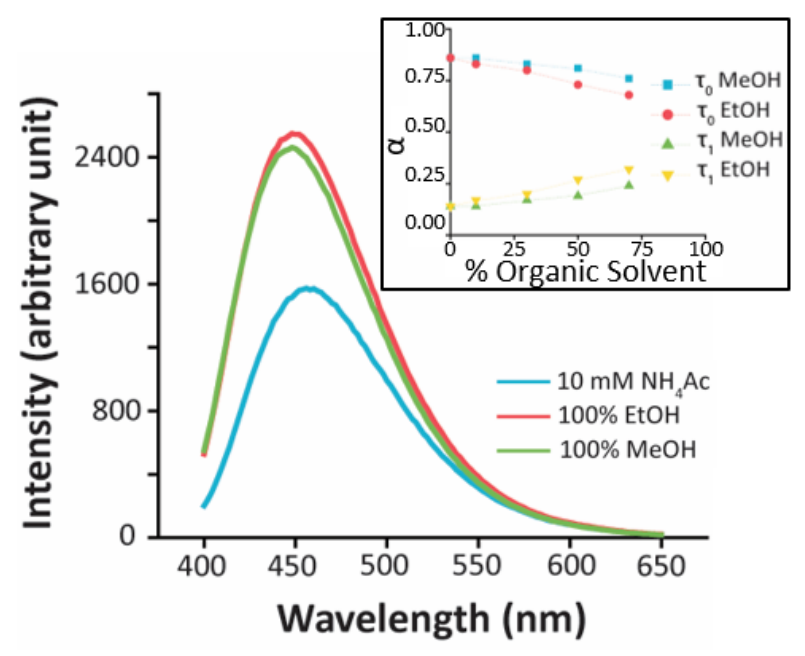


Figure 3.5 Experimental and theoretical IRMPD spectra for the protonated NADH (blue) and $\mathrm{NAD}^{+}$(red) forms. The three lowest energy candidate structures per mobility band and corresponding IR spectra are shown. The boxes represent simplified calculated IR spectra to facilitate the visualization (complete calculated IR spectra are provided in Figure S8).

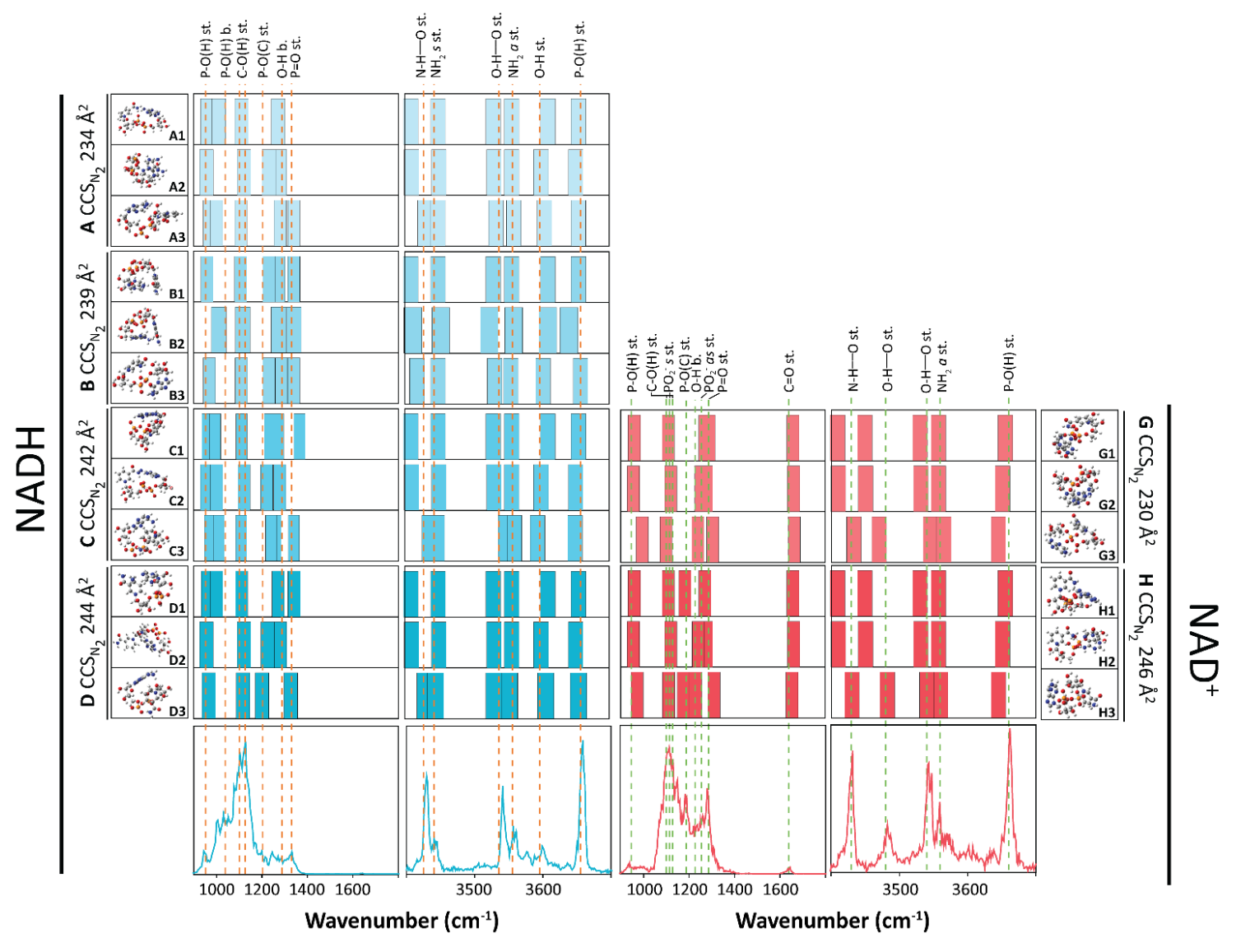




\section{LIST OF REFERENCES}

1. M. Rossmann, Nature, 1976, 262, 726.

2. P. Belenky, K. L. Bogan and C. Brenner, Trends Biochem. Sci., 2007, 32, 12-19.

3. V. Schreiber, F. Dantzer, J. C. Ame and G. de Murcia, Nat. Rev. Mol. Cell Biol., 2006, 7, 517-528.

4. H. H. Hassanain, S. Y. Chon and S. L. Gupta, J. Biol. Chem., 1993, 268, 50775084.

5. P. Bieganowski and C. Brenner, Cell, 2004, 117, 495-502.

6. Q. Zhang, D. W. Piston and R. H. Goodman, Science, 2002, 295, 1895-1897.

7. A. Wilkinson, J. Day and R. Bowater, Mol. Microbiol., 2001, 40, 1241-1248.

8. J. S. Smith and J. D. Boeke, Genes Dev., 1997, 11, 241-254.

9. J. Rutter, M. Reick, L. C. Wu and S. L. McKnight, Science, 2001, 293, 510-514.

10. A. Burkle, Bioessays, 2001, 23, 795-806.

11. R. M. Anderson, K. J. Bitterman, J. G. Wood, O. Medvedik, H. Cohen, S. S. Lin, J. K. Manchester, J. I. Gordon and D. A. Sinclair, J. Biol. Chem., 2002, 277, 18881-18890.

12. A. Katoh and T. Hashimoto, Front. Biosci., 2004, 9, 1577-1586.

13. N. Pollak, C. Dolle and M. Ziegler, Biochem. J., 2007, 402, 205-218.

14. J. Luo, A. Y. Nikolaev, S. Imai, D. Chen, F. Su, A. Shiloh, L. Guarente and W. Gu, Cell, 2001, 107, 137-148.

15. H. Vaziri, S. K. Dessain, E. Ng Eaton, S. I. Imai, R. A. Frye, T. K. Pandita, L. Guarente and R. A. Weinberg, Cell, 2001, 107, 149-159.

16. J. S. Smith, C. B. Brachmann, I. Celic, M. A. Kenna, S. Muhammad, V. J. Starai, J. L. Avalos, J. C. Escalante-Semerena, C. Grubmeyer, C. Wolberger and J. D. Boeke, Proc. Natl. Acad. Sci. USA, 2000, 97, 6658-6663.

17. J. Landry, A. Sutton, S. T. Tafrov, R. C. Heller, J. Stebbins, L. Pillus and R. Sternglanz, Proc. Natl. Acad. Sci. USA, 2000, 97, 5807-5811.

18. S. Imai, C. M. Armstrong, M. Kaeberlein and L. Guarente, Nature, 2000, 403, 795800. 
19. S. J. Lin and L. Guarente, Curr. Opin. Cell Biol., 2003, 15, 241-246.

20. B. M. Bakker, K. M. Overkamp, A. J. van Maris, P. Kotter, M. A. Luttik, J. P. van Dijken and J. T. Pronk, FEMS Microbiol. Rev., 2001, 25, 15-37.

21. M. J. MacDonald and L. K. Marshall, Arch. Biochem. Biophys., 2000, 384, 143153.

22. L. A. Sanni, C. Rae, A. Maitland, R. Stocker and N. H. Hunt, Am. J. Pathol., 2001, $159,1105-1112$.

23. R. Ramasamy, N. Trueblood and S. Schaefer, Am. J. Physiol., 1998, 275, H195203.

24. P. D. Mongan, J. Capacchione, S. West, J. Karaian, D. Dubois, R. Keneally and P. Sharma, Am. J. Physiol. Heart Circ. Physiol., 2002, 283, H1634-1644.

25. A. Gaikwad, D. J. Long, 2nd, J. L. Stringer and A. K. Jaiswal, J. Biol. Chem., 2001, 276, 22559-22564.

26. J. M. Salmon, E. Kohen, P. Viallet, J. G. Hirschberg, A. W. Wouters, C. Kohen and B. Thorell, Photochem. Photobiol., 1982, 36, 585-593.

27. B. R. Masters, A. K. Ghosh, J. Wilson and F. M. Matschinsky, Invest. Ophthalmol. Vis. Sci., 1989, 30, 861-868.

28. A. P. Koretsky, L. A. Katz and R. S. Balaban, Am. J. Physiol., 1987, 253, H856862.

29. B. Chance and M. Lieberman, Exp. Eye Res., 1978, 26, 111-117.

30. D. M. Jameson, V. Thomas and D. M. Zhou, Biochim. Biophys. Acta, 1989, 994, 187-190.

31. T. G. Scott, R. D. Spencer, N. J. Leonard and G. Weber, J. Am. Chem. Soc., 1970, 92, 687-695.

32. Y. D. Wu and K. N. Houk, J. Am. Chem. Soc., 1991, 113, 2353-2358.

33. J. J. Pavelites, J. Gao, P. A. Bash and A. D. Mackerell, J. Comput. Chem., 1997, 18, 221-239.

34. P. E. Smith and J. J. Tanner, J. Mol. Recogn., 2000, 13, 27-34.

35. J. C. Molano-Arevalo, D. R. Hernandez, W. G. Gonzalez, J. Miksovska, M. E. Ridgeway, M. A. Park and F. Fernandez-Lima, Anal. Chem., 2014, 86, 10223-10230. 
36. E. R. Schenk, R. Almeida, J. Miksovska, M. E. Ridgeway, M. A. Park and F. Fernandez-Lima, J. Am. Soc. Mass Spectrom., 2015, 26, 555-563.

37. E. R. Schenk, M. E. Ridgeway, M. A. Park, F. Leng and F. Fernandez-Lima, Anal. Chem., 2014, 86, 1210-1214.

38. E. R. Schenk, V. Mendez, J. T. Landrum, M. E. Ridgeway, M. A. Park and F. Fernandez-Lima, Anal. Chem., 2014, 86, 2019-2024.

39. O. Hernandez, S. Isenberg, V. Steinmetz, G. L. Glish and P. Maitre, J. Phys. Chem. A, 2015, 119, 6057-6064.

40. A. Masson, M. Z. Kamrath, M. A. Perez, M. S. Glover, U. Rothlisberger, D. E. Clemmer and T. R. Rizzo, J. Am. Soc. Mass Spectrom., 2015, 26, 1444-1454.

41. S. Lee, S. J. Valentine, J. P. Reilly and D. E. Clemmer, Int. J. Mass Spectrom., 2012, 309, 161-167.

42. S. Warnke, C. Baldauf, M. T. Bowers, K. Pagel and G. von Helden, J. Am. Chem. Soc., 2014, 136, 10308-10314.

43. N. C. Polfer, J. J. Valle, D. T. Moore, J. Oomens, J. R. Eyler and B. Bendiak, Anal. Chem., 2006, 78, 670-679.

44. J. S. Prell, T. M. Chang, J. A. Biles, G. Berden, J. Oomens and E. R. Williams, J. Phys. Chem. A, 2011, 115, 2745-2751.

45. P. Kupser, K. Pagel, J. Oomens, N. Polfer, B. Koksch, G. Meijer and G. von Helden, J. Am. Chem. Soc., 2010, 132, 2085-2093.

46. R. J. Plowright, E. Gloaguen and M. Mons, ChemPhysChem, 2011, 12, 1889-1899.

47. Flanagan, L. A. Hewlett-Packard Company: Palo Alto, CA; U.S. Patent No. 5872357 A, February 16, 1999; p 19.

48. D. R. Hernandez, J. D. Debord, M. E. Ridgeway, D. A. Kaplan, M. A. Park and F. Fernandez-Lima, Analyst, 2014, 139, 1913-1921.

49. F. A. Fernandez-Lima, D. A. Kaplan and M. A. Park, Rev. Sci. Instr., 2011, 82, 126106.

50. F. A. Fernandez-Lima, D. A. Kaplan, J. Suetering and M. A. Park, Int. J. Ion Mobil. Spectrom., 2011, 14, 93-98.

51. E. W. McDaniel and E. A. Mason, Mobility and diffusion of ions in gases, John Wiley and Sons, Inc., New York, New York, 1973. 
52. R. D. Spencer, J. Chem. Phys., 1970, 52, 1654.

53. J. M. Beechem, Chem. Phys. Lipids, 1989, 50, 237-251.

54. J. R. Lakowicz, Principles of Fluorescence Spectroscopy, Springer, New York, U. S. A., 2010.

55. J. M. Bakker, T. Besson, J. Lemaire, D. Scuderi and P. Maitre, J. Phys. Chem. A, 2007, 111, 13415-13424.

56. J. M. Bakker, R. K. Sinha, T. Besson, M. Brugnara, P. Tosi, J. Y. Salpin and P. Maitre, J. Phys. Chem. A, 2008, 112, 12393-12400.

57. R. K. Sinha, E. Nicol, V. Steinmetz and P. Maitre, J. Am. Soc. Mass Spectrom., 2010, 21, 758-772.

58. R. Prazeres, F. Glotin, C. Insa, D. A. Jaroszynski and J. M. Ortega, Eur. Phys. J. D, 1998, 3, 87-93.

59. F. A. Fernandez-Lima, H. Wei, Y. Q. Gao and D. H. Russell, J. Phys. Chem. A, $2009,113,8221-8234$.

60. Y. Duan, C. Wu, S. Chowdhury, M. C. Lee, G. Xiong, W. Zhang, R. Yang, P. Cieplak, R. Luo, T. Lee, J. Caldwell, J. Wang and P. Kollman, J. Comput. Chem., 2003, 24, 1999-2012.

61. E. Krieger and G. Vriend, Bioinformatics, 2014, 30, 2981-2982.

62. A. D. Bochevarov, E. Harder, T. F. Hughes, J. R. Greenwood, D. A. Braden, D. M. Philipp, D. Rinaldo, M. D. Halls, J. Zhang and R. A. Friesner, Int. J. Quantum Chem., 2013, 113, 2110-2142.

63. I. M. Alecu, J. Zheng, Y. Zhao and D. G. Truhlar, J. Chem. Theor. Comput., 2010, 6, 2872-2887.

64. M. F. Mesleh, J. M. Hunter, A. A. Shvartsburg, G. C. Schatz and M. F. Jarrold, J. Phys. Chem., 1996, 100, 16082-16086.

65. A. A. Shvartsburg and M. F. Jarrold, Chem. Phys. Lett., 1996, 261, 86-91.

66. I. Campuzano, M. F. Bush, C. V. Robinson, C. Beaumont, K. Richardson, H. Kim and H. I. Kim, Anal. Chem., 2011, 84, 1026-1033.

67. C. Larriba and C. J. Hogan, J. Comput. Phys., 2013, 251, 344-363.

68. C. Larriba and C. J. Hogan, Jr., J. Phys. Chem. A, 2013, 117, 3887-3901. 
69. U. C. Singh and P. A. Kollman, J. Comput. Chem., 1984, 5, 129-145.

70. B. H. Besler, K. M. Merz and P. A. Kollman, J. Comput. Chem., 1990, 11, 431439.

71. N. A. Pierson, S. J. Valentine and D. E. Clemmer, J. Phys. Chem. B, 2010, 114, 7777-7783.

72. A. L. Patrick, C. N. Stedwell and N. C. Polfer, Anal. Chem., 2014, 86, 5547-5552.

73. T. N. Wassermann, O. V. Boyarkin, B. Paizs and T. R. Rizzo, J. Am. Soc. Mass Spectrom., 2012, 23, 1029-1045.

74. E. E. Baquero, W. H. James, 3rd, S. H. Choi, S. H. Gellman and T. S. Zwier, J. Am. Chem. Soc., 2008, 130, 4784-4794.

75. K. Jeanne Dit Fouque, H. Lavanant, S. Zirah, V. Steinmetz, S. Rebuffat, P. Maitre and C. Afonso, J. Phys. Chem. A, 2016, 120, 3810-3816.

76. C. Nadolny and G. Zundel, J. Mol. Struct., 1996, 385, 81-87. 


\section{CHAPTER 4.}

\section{CHARACTERIZATION OF INTRAMOLECULAR INTERACTIONS OF CYTOCHROME $C$ USING HYDROGEN DEUTERIUM EXCHANGE - TRAPPED ION MOBILITY - MASS SPECTROMETRY AND MOLECULAR DYNAMICS}

This chapter was published in Analytical Chemistry and adapted with permission from all authors.

J. C. Molano-Arevalo, K. Jeanne Dit Fouque, K. Pham, J. Miksovska, M. E. Ridgeway, M. A. Park, F. Fernandez-Lima. Anal. Chem., 2017, 89 (17), 8757-8765 


\begin{abstract}
Globular proteins, such as cytochrome c (cyt c), display an organized native conformation, maintained by a hydrogen bond interaction network. In the present work, the structural interrogation of kinetically trapped intermediates of cyt c was performed by correlating the ion-neutral collision cross section (CCS) and charge state with the starting solution conditions and time after desolvation using collision induced activation (CIA), time resolved hydrogen/deuterium back exchange (HDX) and trapped ion mobility spectrometry - mass spectrometry (TIMS-MS). The high ion mobility resolving power of the TIMS analyzer allowed the identification of new ion mobility bands, yielding a total of 63 mobility bands over the +6 to +21 charge states and 20 mobility bands over the -5 to 10 charge states. Mobility selected HDX rates showed that for the same charge state, conformers with larger CCS present faster HDX rates in both positive and negative ion mode, suggesting that the accessible surface area defines the exchange rate regardless of the charge state. Complementary molecular dynamic simulations permitted the generation of candidate structures and a mechanistic model of the folding transitions from native (N) to molten globule (MG) to kinetic intermediates (U) pathways. Our results suggest that cyt c major structural unfolding is associated with the distancing of the $\mathrm{N}$ - and $\mathrm{C}$ - terminal helices and subsequent solvent exposure of the hydrophobic, heme-containing cavity.
\end{abstract}




\section{INTRODUCTION}

Cytochrome $c$ (cyt $c$ ) has been widely investigated using mass spectrometry based techniques, and it has become an intriguing system for ion mobility spectrometry (IMS) and hydrogen/deuterium exchange (HDX) experiments. The appeal for IMS-based studies of cyt $c$ comes from its involvement in key cellular processes, ${ }^{1-4}$ and the interactions of the protein with multiple substrates and/or intracellular proteins, which in turn are associated with subtle changes in conformation. These conformational changes include fluctuations in the heme iron coordination, and tertiary and secondary structure alterations, suggesting that cyt $c$ can populate different conformational species under native conditions. ${ }^{5-9}$ With the development of soft ionization sources (e.g., electrospray ionization, ESI), the evaporative cooling of the solvent can lead to a freezing of multiple stable conformations,

otherwise known as the "memory effect". ${ }^{10,11}$ Although this effect is solvent and biomolecule dependent, previous works have reported the benefits of studying the conformational space as a function of the charge heterogeneity, the solvent conditions, as well as the transitions as a function of the bath gas collision partner and ion effective temperature. ${ }^{12-16}$ For example, the unfolding of cyt $c$, induced with denaturing conditions, was examined by Konermann et al. ${ }^{15}$ using a combination of ESI and solution-based techniques revealing that a decrease in $\mathrm{pH}$ induced a cooperative unfolding transition accompanied by a disruption of the secondary and tertiary structure. Mass spectra analysis, obtained as a function of the starting solution conditions, showed that the transition from low to high charge states was due to the breakdown of the tertiary structure. Early studies on cyt $c$ using a drift tube, conducted by Jarrold and co-workers, ${ }^{17}$ showed that, for the 
lower charge states (centered at $+7,+8$ ), the collision cross sections (CCS) of cyt $c$ were consistent with those expected from native structure found in solution (PDB: 1HRC, Xray $\left.^{18}\right)$. The intermediate charge states displayed metastable structures that unfolded upon heating, while the higher charge states (centered at $+15,+16$ ) exhibited CCS related to extended conformations.

Moreover, complementary structural studies were performed on cyt $c$ in the gas-phase by McLafferty and co-workers ${ }^{19}$ using a Fourier-transform ion-cyclotron-resonance mass spectrometer (FT-ICR-MS) and HDX experiments. They showed that HDX exchange of cyt $c$ in the gas-phase occurs only in three different reactive forms, while in solution the reaction involves nearly all labile hydrogen atoms because of a fast dynamic equilibrium between conformers. The mass spectra of the deuterated +12 to +14 charges states exhibited peak splitting, indicating that two different ionic forms of identical molecular mass had different numbers of reactive hydrogens. These values were also observed for other charge states with 74 reactive hydrogens for the +12 to $+16,113$ for the +8 to +14 , and 53 for the +6 and +7 charge states. Later on, McLafferty and coworkers ${ }^{20}$ have reported at least six different states for gaseous cyt $c$ ions based on their accessible sites for exchange. They also found that manipulation of these ions by infrared radiation, high velocity collisions, or proton stripping, can alter the levels of exchange in discrete steps.

Using drift tube IMS experiments, Clemmer and coworkers ${ }^{21}$ showed that it is possible to study specific conformers of cyt $c$ by varying the voltage used to inject the ions into the drift tube. The number of exchangeable hydrogen atoms was independent of the charge state for diffuse conformers obtained at high injection voltages (63 of a possible 198) while 
the number of exchangeable hydrogens for the compact conformers, observed for the +8 to +10 charge states, was 46 . These results suggested that compact structures protect some hydrogens in the gas-phase and that many sites that exchange in solution are restricted for exchange in the gas-phase, even for open conformers. Douglas and co-workers ${ }^{22}$ investigated the conformational transitions of cyt $c$ induced by denaturing conditions using HDX followed by ESI-MS and showed that the exchange rate is equal for intermediates found in both contributions of a bimodal charge state distribution. These results suggested that the intermediates are very flexible and may involve two or more rapidly interconverting conformers. They also found that at high concentrations of methanol (i.e., 90\% $\mathrm{MeOH}$ ), cyt $c$ denatures into non-native helices, which protect against HDX in a similar manner to the native conformation. A later study from Douglas and coworkers ${ }^{23}$ displayed the expanded denatured states and expanded helical denatured states of cyt $c$ generated from solutions with $50 \%$ and $90 \% \mathrm{MeOH}$, respectively. They showed no differences in CCS and HDX for ions of a given charge state of cyt $c$ for either the expanded or helical expanded denatured states. Clemmer's group later studies ${ }^{24}$ on the HDX temperature dependence of compact (+5) and elongated (+9) cyt $c$ showed that the exchange rates of HDX in the gas-phase decrease an order of magnitude when the exchange occurs at high temperature (i.e., $>400 \mathrm{~K})$. The exchange levels of compact $(+5)$ and elongated (+9) conformers at $300 \mathrm{~K}$ were 53 and 63, respectively. At temperature values greater than $335 \mathrm{~K}$, the levels increase to $\sim 200$ for the +5 charge state and $\sim 190$ for the +9 charge state while the CCS remained invariant at all temperatures. Beauchamp and coworkers ${ }^{25}$ suggested that the proximity between the charge site and exchange site are important factors in the exchange profiles of both conformers. Previous studies have also 
reported the independence between the charge states with the levels of HDX exchange in the gas-phase. ${ }^{19-21,26}$ As noted by Clemmer and coworkers ${ }^{21}$, HDX numbers appear to depend strongly on the instrumentation and operating conditions used. Moreover, when CCS and HDX values are complemented with molecular dynamics (MD), candidate structures can be proposed for every kinetically trapped intermediate species observed. ${ }^{27-}$ 30

With the recent introduction of trapped ion mobility spectrometry coupled to mass spectrometry (TIMS-MS), ${ }^{31-35}$ and more recently complemented with hydrogen/deuterium back exchange (HDX-TIMS-MS) ${ }^{36}$ the mobility measurement and number of HDX back exchanges can be performed simultaneously as a function of time after desolvation. TIMS high resolving power ( $\mathrm{Rp}$ up to 400$)^{37-39}$ combined with the possibility to measure kinetic intermediates and to perform collision induced activation (CIA) prior to the TIMS analysis, provides a powerful tool for the analysis of biomolecules. ${ }^{40-43}$

In the present work, we explore for the first time the potential of CIA-HDX-TIMS-MS to study the kinetically trapped intermediates of cyt $c$ by populating the conformational free energy landscape as a function of the starting solution conditions (e.g., organic content and $\mathrm{pH}$ ) and as a function of the CIA prior to the HDX-TIMS-MS measurements. MD simulations were used to propose candidate structures for each kinetically trapped intermediate observed and a mechanistic model of the folding transitions from native to molten globule to kinetic intermediates is proposed. 


\section{EXPERIMENTAL SECTION}

Materials and reagents

Horse heart cyt $c$ (C2506) was purchased from Sigma-Aldrich (St. Louis, MO). All solvents used in these studies were analytical grade or better and purchased from Fisher Scientific (Pittsburg, PA). Cyt $c$ stock was prepared in $10 \mathrm{mM}$ ammonium acetate $\left(\mathrm{NH}_{4} \mathrm{Ac}\right)$ buffer for the positive ion mode experiments, dialyzed against the same buffer and diluted to a final concentration of $0.5,1,5$, and $10 \mu \mathrm{M}$ in: A) $10 \mathrm{mM} \mathrm{NH}_{4} \mathrm{Ac}(\mathrm{pH} 7.1$ ), B) $95: 5$ and 50:50 (v/v) $\mathrm{H}_{2} \mathrm{O} / \mathrm{MeOH}(\mathrm{pH} 4.6)$, and C) 94:5:1, 89:10:1, 79:20:1, 69:30:1, 59:40:1 and 49:50: $1 \mathrm{H}_{2} \mathrm{O} / \mathrm{MeOH} / \mathrm{CH}_{3} \mathrm{COOH}(\mathrm{pH} 3.3)$. In the case of the negative ion mode analysis, a stock solution of $100 \mu \mathrm{M}$ was used and then diluted to $40: 60$ (v/v) $\mathrm{H}_{2} \mathrm{O} / \mathrm{MeOH}$, and 40:50:10 $\mathrm{H}_{2} \mathrm{O} / \mathrm{TFE} / \mathrm{Et}_{3} \mathrm{~N}$. For the HDX experiments operating in the positive ion mode, cyt $c$ was prepared in $\mathrm{D}_{2} \mathrm{O}$ (Sigma-Aldrich, 151882) at $1 \mu \mathrm{M}(\mathrm{pH} 7.02)$ incubated for $3 \mathrm{~h}$ and $48 \mathrm{~h}$ at $37{ }^{\circ} \mathrm{C}$ and $0.1 \% \mathrm{CH}_{3} \mathrm{COOH}$ was added to solutions prior to the HDX-TIMS-MS analysis (pH 6.88). For HDX experiments operating in the negative ion mode, cyt $c$ was incubated in $\mathrm{D}_{2} \mathrm{O}$ at $50 \mu \mathrm{M}$. Nano-ESI emitters were pulled from quartz capillaries (O.D.: $1.0 \mathrm{~mm}$ and I.D.: $0.70 \mathrm{~mm}$ ) using Sutter Instruments Co. P2000 laser puller. Lowconcentration Tuning Mix calibration standard (TuneMix, G24221A) was purchased from Agilent Technologies (Santa Clara, CA).

\section{CIA-HDX-TIMS-MS}

Details regarding the TIMS operation compared to traditional IMS can be found elsewhere. ${ }^{32,33,41,44,45}$ Briefly, TIMS mobility separation is based on holding the ions 
stationary using an electric field against a moving buffer gas. ${ }^{46}$ During HDX-TIMS-MS experiments, the rate of HDX back exchange is measured as a function of the trapping time.$^{36}$ That is, in HDX-TIMS-MS deuterated, molecular ions are introduced into the TIMS cell and they can undergo back exchange reaction with residual molecules of the bath gas. The rate of HDX can be tuned by changing the velocity of the gas and the trapping time while simultaneously measuring the elution voltage directly related to the CCS as shown below.

The mobility, $K$, of an ion in a TIMS cell is described by:

$K=\frac{v_{g}}{E}=\frac{A}{\left(V_{\text {elution }}-V_{\text {out }}\right)}$

where $v_{g}, E, V_{\text {elution }}$ and $V_{\text {out }}$ are the gas velocity, applied electric field, elution voltage and base voltage, respectively. The constant $A$ was determined using a Tuning Mix calibration standards $\left(m / z, 322 K_{o}=1.376 \mathrm{~cm}^{2} \mathrm{~V}^{-1} \mathrm{~s}^{-1}, m / z 622 K_{o}=1.013 \mathrm{~cm}^{2} \mathrm{~V}^{-1} \mathrm{~s}^{-1}\right.$, and $m / z, 922 \mathrm{~K}_{\mathrm{o}}=$ $\left.0.835 \mathrm{~cm}^{2} \mathrm{~V}^{-1} \mathrm{~s}^{-1}\right) .^{33,47}$

The same RF ( $880 \mathrm{kHz}$ and $280 \mathrm{Vpp}$ ) was applied to all electrodes including the entrance funnel, the ion mobility separating section, and the exit funnel (Figure S4.1). A custommade nano-electrospray ionization source was used for all the analyses. TIMS separation was performed using nitrogen as a bath gas at $300 \mathrm{~K}$, and the gas flow velocity was held constant in all the experiments $\left(P_{1}=2.6\right.$ and $\left.P_{2}=1.0 \mathrm{mbar}\right) . P_{1}$ and $P_{2}$ values were held constant for all experiments. A fill/trap/ramp/wait sequence of 1-10/1-10/5-500/50 ms was used and an average mobility resolution of $180-250$ was observed. A total of 500 accumulations and 10 frames were acquired per TIMS experiment. 
Mobility values $(K)$ were correlated with $\operatorname{CCS}\left(\Omega, \AA^{2}\right)$ using the following equation:

$\Omega=\frac{(18 \pi)^{1 / 2}}{16} \frac{z}{\left(k_{B} T\right)^{1 / 2}}\left(\frac{1}{m_{I}}+\frac{1}{m_{b}}\right)^{1 / 2} \frac{1}{K} \frac{760}{P} \frac{T}{273.15} \frac{1}{N^{*}}$

where $z$ is the charge of the ion, $\mathrm{k}_{\mathrm{B}}$ is the Boltzmann constant, $N^{*}$ is the number density and $m_{I}$ and $m_{b}$ refer to the masses of the ion and bath gas, respectively. ${ }^{46}$

The number of HDX back-exchanges is obtained by the mass shift relative to the nondeuterated protein (Figure S4.2). Collision induced activation were performed prior to the TIMS-MS by varying the electric field between the capillary outlet ( $\left.V_{\text {cap }}: 50-190 \mathrm{~V}\right)$, deflector plate $\left(V_{\text {def }}: 60-200 \mathrm{~V}\right)$ and funnel entrance $\left(V_{\text {fun }}: 0-150 \mathrm{~V}\right)$ in $10 \mathrm{~V}$ steps.

Theoretical method

A candidate structure generation algorithm was used to sample cyt $c$ conformational space. ${ }^{48}$ The 1 HRC protein data bank entry for cyt $c$ was utilized as the starting structure. $^{18,49}$ Briefly, molecular dynamics simulations were used to reproduce the experimental conditions and to generate the identity vectors that define cyt $c$ conformational space. This approach is similar to that previously described by FernándezLima et $a l .{ }^{13}$ for peptides, with the main characteristic that the initial search targets the generation of the identity vectors, followed by charge assignment and energy minimization. Once the candidate structures were identified for each IMS band, charge assignment was performed by scoring the accessible surface area based on the score of the amino acid residues. ${ }^{50,51}$ For example, solvent accessibility and the pKa of the acidic and basic residues were primarily used to assign the protonation and de-protonation sites. It is known that 
charge localization can influence electrostatic interactions and therefore the conformational dynamics of molecular ions. ${ }^{52,53}$ To account for the charge state influence on the CCS, energy optimization steps were performed following the charge assignments. All simulations were performed in a NVT thermostat using AMBER03 force field in YASARA software. The molecular dynamic simulations yielded identity vectors that cover the +6 to +13 distribution and the theoretical $\mathrm{CCS}_{\mathrm{N} 2}$ for each structure were calculated using the TM algorithm implemented in the IMoS software. ${ }^{54-56}$

\section{RESULTS AND DISCUSSION}

The mass spectrometry analysis of cyt $c$ in positive and negative ion mode displayed a charge state distribution dependence with the solvent conditions in agreement with previous studies. ${ }^{15,22}$ Under native conditions (e.g. $0 \% \mathrm{MeOH}, 10 \mathrm{mM} \mathrm{NH}_{4} \mathrm{Ac}$ ), a narrow distribution of charge states centered at +7 was observed (Figure 4.1, left panel on the top). An increase in the concentration of $\mathrm{MeOH}$ to $5 \%$ showed a conformational change in the protein, represented by a bimodal distribution with two envelopes centered at +9 and +17 . Further increases in the concentration of $\mathrm{MeOH}$ decreased the relative intensity of the envelope for the lower native-like charge states (e.g. +6 to +9$)$ and a single distribution was observed at $40 \% \mathrm{MeOH}$ centered at +17 . For the negative ion mode, the -5 and -6 charge states were observed for a solution with $40 \% \mathrm{MeOH}$ (Figure 4.1, left panel on the bottom). A change in the starting solvent condition using 50\% TFE and $10 \% \mathrm{Et}_{3} \mathrm{~N}$ showed a conformational change in the cyt $c$ represented by a different charge state distribution centered at -8 . To confirm the conformational diversity of cyt $c$ under different solution conditions, ultraviolet-visible (UV-VIS) spectroscopy was used to trace the Soret band 
intensity to monitor the accessibility of solvent to the heme group as the band shifts from 400 to $395 \mathrm{~nm}$ after acidification of the solution (Figure S4.3). As previously described by Konermann et al.,${ }^{15}$ in aqueous solutions of low ionic strength, cyt $c$ is in the native state and the heme iron is coordinated by the strong-field ligands His 18 and Met80 residues, which produces a low spin complex with a Soret absorption maximum between 400 and $410 \mathrm{~nm} \cdot{ }^{18,57-61}$ Further acidification of the solution or an increase in the $\mathrm{MeOH}$ concentration induces a cooperative unfolding transition to a molten-globule state, ${ }^{60,62,63}$ and the displacement of both strong-field ligands by weak-field ligands from the solvent (e.g., $\mathrm{H}_{2} \mathrm{O}$ ) produces a high-spin complex with a Soret absorption between 390 and 395 nm..$^{59,60,64}$

IMS profiles for the observed charge states on each condition were normalized to the intensity of each charge state in the mass spectrum (Figure 4.1, right panel). The overall CCS profiles (Figure 4.1, black lines) obtained by summation of the ion mobility resolved data (Figure 4.1, color lines), exhibited a distribution of conformations from native (Figure 4.1, red background) to molten globular (Figure 4.1, blue background), and finally to the denatured states trapped as kinetic intermediates (Figure 4.1, yellow background). Ion mobility experiments were performed as a function of the starting solution conditions and the collision induced activation (CIA) energy (Figure 4.2) in the positive ion mode. Soft activation energy conditions were used to preserve the conformation from the solution (e.g., $V_{\text {cap }}: 50 \mathrm{~V} ; V_{\text {def: }} 60 \mathrm{~V} ; V_{\text {fun }}: 0 \mathrm{~V}$ ). In addition, TIMS analyzers permit the study of the temporal evolution of the IMS distribution as a function of the time after the molecular ions are formed during the nanoESI process (e.g., trapping time of 100-500 ms after 
desolvation). Inspection of the IMS bands as a function of the trapping time did not show variation in terms of the relative abundance. However, previous reports have shown variation of the IMS profiles of cyt $c$ at shorter times after desolvation. ${ }^{29}$ This result suggests that the observed IMS bands in TIMS correspond to stable "desolvated", kinetically trapped intermediates. As displayed in the mass spectrometry analysis, the IMS profiles for the +8 to +11 charge states are obtained when the starting solution contains at least $5 \% \mathrm{MeOH}$, suggesting that a solution only with $10 \mathrm{mM} \mathrm{NH}_{4} \mathrm{Ac}$ cannot induce the necessary conformational changes that allows basic sites to be protonated during the nanoESI process. The overall IMS profiles for all the solution conditions are shown next to the contour plots, where IMS bands are labeled for the kinetically trapped intermediates (Figure 4.2, left panel). Most IMS profiles are uniform as a function of the starting solution conditions. Interestingly, the contour plot for the +9 charge state is broad and displays heterogeneity in the relative intensity of B and C, whereas an increase is observed after the addition of $20 \% \mathrm{MeOH}$ to the solution (Figure 4.2, left panel). We interpret this result as evidence of kinetic intermediates involved in the transition from native-like to molten globular conformation. In a similar way, the IMS profiles for the +10 , label $\mathrm{B}$, and +11 , labels B and C, charge states are more intense when the solution contains $30 \%$ and $20 \%$ $\mathrm{MeOH}$ respectively.

Another way to probe the conformational space of cyt $c$ in the gas phase is to activate the ions prior to IMS analysis (Figure 4.2, right panel). Note that the label in the CIA plots only shows the deflector voltage $\left(V_{d e f}\right)$ as the variable, but the voltage of the both capillary $\left(V_{c a p}\right)$ and entrance funnel $\left(V_{f u n}\right)$ regions were increased accordingly using $10 \mathrm{~V}$ increments 
per experiment. The solution used for these experiments was $10 \mu \mathrm{M}$ of cyt $c$ in $10 \mathrm{mM}$ $\mathrm{NH}_{4} \mathrm{Ac}, 5 \% \mathrm{MeOH}$, and $1 \% \mathrm{CH}_{3} \mathrm{COOH}$. Examination of the contour plots exhibited little variation in the IMS profiles for the $+6,+10$, and +11 charge states as a function of the activation energy (Figure 4.2, right panel). It is possible that the energy used to activate the ions is not enough to disrupt the intramolecular interactions from the hydrogen bond network that holds together the compact native-like conformations observed in the +6 charge

state. The conformations for the +10 and +11 charge states are already open, and the additional energy does little to populate more extended structures. However, it is interesting to note the changes in the intensity of the IMS bands (labels D and E) for the +10 charge state relative to the to the solution obtained profile, suggesting that, even though all the conformers are observed, the open conformations are more stable and abundant when the activation takes place. Inspection of the contour plot for the +7 charge state showed a broad band, which combines the compact conformers A and B (Figure 4.2, right panel). A stepwise transition between conformations $\mathrm{C}$ through $\mathrm{F}$ end in the trapping of two new open conformers (labels $\mathrm{G}$ and $\mathrm{H}$ ) when the CIA energy is high (i.e., $V_{\text {cap }}$ : $130 \mathrm{~V}$; $V_{\text {def: }} 140 \mathrm{~V} ; V_{\text {fun }}: 80 \mathrm{~V}$ ). The IMS profile for the +8 charge state showed the trapped intermediates observed in solution (labels A and B) and two generated as the CIA energy increases and the trapped intermediates are allowed to climb the energy funnel barriers and populate different local minima (i.e., labels $\mathrm{C}$ and D at $V_{\text {cap }}: 80 \mathrm{~V} ; V_{\text {def: }} 90 \mathrm{~V} ; V_{\text {fun }}: 30 \mathrm{~V}$; and label E at $\left.V_{\text {cap }}: 140 \mathrm{~V} ; V_{\text {def. }} 150 \mathrm{~V} ; V_{\text {fun }}: 90 \mathrm{~V}\right)$. The IMS profile for the +9 charge state also displays a broad transition between conformers as the solution conditions changes 
(Figure 4.2, right panel). However, when the ions are activated, it is possible to better distinguish the A, B, and C IMS bands. These results for the $+7,+8$, and +9 charge states show that the transition between compact and open conformers can be induced via CIA. That is, when the internal energy of the protein increases and overcomes the conformational energy barrier, other local minima are accessible. The CCS and $K_{0}$ values are summarized in Table S1.

The high IMS resolving power of the TIMS analyzer allowed to observe a larger number of IMS bands that have been overlooked in previous works for charge states higher than +11 (Figure 4.3 and Table S2). Moreover, inspection of the +12 to +21 charge states exhibited a reduction in the number of IMS bands and narrower distributions when compared to the +8 to +11 charge states. No major differences are found in the IMS profiles for the +12 to +21 charge states as a function of the solution conditions, the activation energy, or the trapping time. Nevertheless, when compared to previous IMS experiments a larger number of IMS bands are now separated using TIMS for the +12 to +21 charge states. Complementary TIMS experiments with varying cyt c concentration (e.g., 0.5, 1, 5, and $10 \mu \mathrm{M})$ rule out the possibility of IMS bands corresponding to the formation of multimeric assemblies. The TIMS data are consistent with previous CCS reports on cyt $c$ using other IMS variants (Figure S4.4).

This discussion can be followed by the inspection of the cyt $c$ back-exchange HDX dynamics in the TIMS analyzer (Figure 4.4). Two HDX incubation time were considered 3 and $48 \mathrm{~h}$ in $\mathrm{D}_{2} \mathrm{O}$ followed by $50 \mathrm{~ms}$ TIMS measurement intervals (Figure S4.5). IMS resolved HDX data were acquired for all the IMS bands of the +6 to +13 charge states. In 
solution, at physiological conditions (e.g., $37^{\circ} \mathrm{C}, \mathrm{pH} 7.0$ ), most molecules of cyt $c$ are probably populating the "native" state conformations (lower minima of the free energy landscape). Local unfolding events in an excess of $\mathrm{D}_{2} \mathrm{O}$ allow the exchange reaction between amide hydrogens and deuterons by any of the three well-known mechanisms (i.e., base catalysis, acid catalysis by $\mathrm{N}$-protonation, and acid catalysis by O-protonation). ${ }^{65-68}$ During the nanoESI process, the cyt $\mathrm{c}$ molecules are protonated in positive ion mode as a function of the degree of exposure of basic residues. If a low amount of acid is added to the solution, the conformation of some of the protein molecules is disturbed from the native state, allowing the fast exchange of usually slow-exchanging hydrogens. Our results showed independence between the initial number of deuterons and the charge state for all the incubation conditions, and the number of initial deuterons was never close to the maximum number of possible exchanges (i.e., 196). For example, HDX-TIMS analysis without trapping $\left(t_{\text {trap }}=0 \mathrm{~ms}\right)$ and with back-exchange trapping $\left(t_{\text {trap }}=100-500 \mathrm{~ms}\right)$ showed that the number of deuterons is independent from the charge state for the two incubation times considered (Figure 4.4). The initial number of deuterons at $48 \mathrm{~h}$ is considerably higher than at $3 \mathrm{~h}$, as many of the non-exposed hydrogen atoms involved in the hydrogen bond network that preserves the protein structure may have exchanged during incubation, most likely due to conformational rearrangements and small movements that expose these otherwise protected atoms. To generate ions of higher charge states, a small amount of acid (i.e., $0.1 \% \mathrm{CH}_{3} \mathrm{COOH}$ ) was added to the incubated solutions ( $\mathrm{pH}$ 6.88). It is possible that the addition of a small amount of acid might be enough to quench, at least in some degree, the exchange reaction between the exposed slow-exchanging hydrogens and the deuterons from the solvent, or, as the quenching is not complete, some exchange 
still occurs when the protein unfolds due the denaturing effect of the acid, which leads to a slight overestimation of the number of initial deuterons for the higher charge states. However, the range of HDX-TIMS back-exchange after $100 \mathrm{~ms}$ is narrower at $48 \mathrm{~h}$ compared to $3 \mathrm{~h}$; this result suggests that some hydrogen atoms can slowly exchange in solution but they are not readily accessible for back-exchange in the gas-phase. Clustering of the number of conformations based on the number of deuterons at $t_{\text {trap }}=100 \mathrm{~ms}$ revealed four major conformational groups (Figure 4.4), which can be associated with different levels of protection (Figure S4.6). It is important to note that small shortcomings of the current approach is that the residual water content cannot be controlled at the moment and measurement differences are observed from sample to sample. However, the experimental design allows a direct comparison of the exchange levels between charge states and mobility bands within the same sample. The HDX-TIMS back-exchange follows a general declining trend (Figure S4.5). For some cases (e.g., +13 charge state) a bimodal distribution is observed, where a fast back exchange occurs within the first $200 \mathrm{~ms}$. Since changes are not observed in the CCS distribution over the trapping time, we interpret the bimodal distribution as a consequence of similar conformations with different charge configuration, and to a lesser extend to minor conformational rearrangements exposing more hydrogens that are not resolved in the CCS domain.

Traditional HDX experiments use the relay-mechanism to explain the isotopic exchange in the gas-phase via charge-mediated exchanges in a timely-efficient manner ${ }^{69-72}$ The HDXTIMS experiments share some analogy with thermal energy HDX ion-molecule reactions used to probe conformational differences by Smith and coworkers, ${ }^{73}$ but HDX-TIMS back- 
exchange ion-neutral reactions are performed at lower pressures (few mbar), larger free mean path, and low energy transfer per collision. The HDX-TIMS back-exchange relies on collisions of the kinetically trapped intermediates with residual water molecules, which can be experimentally tailored by the velocity of the gas or by the amount of residual water in the system. Closer inspection to the back-exchange rates for a given charge state, in both positive and negative ion mode, showed that as the CCS increases faster, HDX rates are observed (Figure 4.4 and Table S1 and S3). Moreover, similar rates are observed for conformers of the same conformational state (e.g., $\mathrm{N}$ or MG) regardless of the charge state or polarity (Figure S4.7). We interpret these results as a consequence of the accessible surface area per conformational state. That is, there are regions on the surface of the protein that allow for the back HDX to occur, probably mediated by a proton. For example, differences in back HDX rates with the CCS per charge state can be related to different charge configurations, which based on the position of the charge and neighboring amino acids will ultimately define the HDX rate. Notice that HDX-TIMS experiments are particularly suitable for the study of cyt $\mathrm{c}$ accessible surface area during its unfolding pathway. Exposed deuterons back-exchange faster while buried deuterons are protected, providing a better insight into the structural dynamics of cyt $\mathrm{c}$ unfolding. While this initial set of experiments only give a qualitative view of the hydrogen network, charge configuration, and the CCS of the cyt c kinetic intermediates, further experiments using top-down strategies on mobility selected HDX time points will permit further assessment of the intramolecular forces that stabilize the cyt $\mathrm{c}$ kinetic intermediates. 
Candidate structures were proposed for all IMS bands (Figure 4.5). Closer inspection to the candidate structures permitted the generation of a mechanistic model of the main intramolecular interactions that define the folding pathways and their intermediates (Figure S4.8). For example, inspection of the candidate structures revealed that major structural differences are associated with the increase in distance between the $\mathrm{N}$ - and $\mathrm{C}$ - terminal helices and the solvent exposure of the heme cavity at higher charge states are consistent with previous results. ${ }^{74-76}$ That is, the +10 charge state structures are characterized by the destabilization of the secondary structure of the $\mathrm{N}$ - and $\mathrm{C}$ - terminal helices and cleavage of the Trp59 and of the Met80 residues and the heme propionate hydrogen bond. It has been reported that $\operatorname{Trp59}$ and Met80 residues provide a unique hydrophobic environment to the heme crevice. ${ }^{77,78}$ Without these interactions, the crevice opens up, exposing Thr78 and Pro71, which are residues involved in the formation and stabilization of the heme crevice due a network of hydrogen bonds around the heme group. ${ }^{79}$ The interface formed by the interaction of the helices occurs

immediately after the covalent binding of the heme group to the polypeptide via thioether bonds. ${ }^{80}$ Closer inspection of the candidate structures for the $+6,+7$, and +8 charge states revealed that the $\pi-\pi$ interaction of Phe10 and Tyr97 aromatic groups is lost followed by the loss of the "peg-in-a-hole" Gly6 and Leu94 interaction, which is also consistent with previous observations. ${ }^{81}$ For example, the +8 charge state of cyt $c$ showed that Gly6 and Leu94 residues are interacting while there is a distancing between Phe10 and Tyr97 residues compared to the structures generated for the +6 and +7 charge states. Moreover, structures for the +8 charge state displayed an increasing separation between the $\mathrm{N}$ - and $\mathrm{C}$ - 
terminal helices while conserving the interaction between Gly6 and Leu94 residues. The +9 charge state of cyt $c$ showed that Gly6 residue is no longer interacting with Leu94 residue, and the +9 conformations exhibited a widening gap between these two amino acids while conserving the general structure of the $\mathrm{N}$ - and $\mathrm{C}$ - terminal helices. It should be noted that when compared to the myoglobin folding/unfolding pathway, ${ }^{36}$ the covalent attachment of the heme in cyt $c$ stabilizes the MG and $\mathrm{U}$ intermediate states (less structural flexibility) into more defined IMS bands.

\section{CONCLUSIONS}

The high mobility resolving power of the TIMS analyzer allowed the identification of new ion mobility bands for cyt $\mathrm{c}$, yielding a total of 63 mobility bands over the +6 to +21 charge states and 20 mobility bands over the -5 to -10 charge states. Experimental results showed that only 5\% methanol and $1 \%$ acetic acid can disrupt the intramolecular interactions (i.e., hydrogen bond network) that holds together the integrity of the native conformation. TIMS-MS experiments enabled us to establish a general trend where the trapped intermediates increase in CCS as a function of the charge state and the inspection of the candidate structures proposed for the lower charge states (i.e., +6 and +7 , and -5 and -6 )

confirmed that the solution "native" states are retained in the gas-phase. For the first time using a TIMS analyzer, we describe how the collisional activation of the desolvated ions permitted the generation of other conformational states of cyt $c$ that are not readily accessible by varying the starting solution. Our results suggest that major structural unfolding motifs were associated with the distance between the $\mathrm{N}$ - and $\mathrm{C}$ - terminal helices and the solvent exposure of the heme cavity. 
The possibility of measuring CCS and HDX back-exchange rates simultaneously, permitted the identification of local fluctuations which might later be useful in the identification of features that define the structure of cyt c kinetically trapped intermediates. The hydrogens exposed in the surface of the protein, which can be partially involved (or not) in the hydrogen bond interaction network, are readily exchangeable. The effect of the rearrangement of the contact points between secondary structures, which disrupts the hydrogen bond interaction network but keep the overall integrity of the protein, allows the identification of the number of hydrogens completely buried that are not going to exchange because of their role preserving the native conformation of the protein. In the case of cyt $c$, changes in the HDX rates were observed as a function of the CCS for the same charge state, which can be related to different charge configurations. The HDX-TIMS-MS experiments provide a powerful analytical tool for the study of protein folding and intermediates. While major advances are highlighted in the present case, further improvements by performing top-down experiments in HDX-TIMS selected intermediates will provide a more detailed description on the charge configuration for more accurate candidate structure generation.

\section{ASSOCIATED CONTENT}

Scheme of the TIMS cell, experimental and theoretical mass shift for the deuterated and non-deuterated +6 and +13 charge states, UV-VIS spectroscopy in the $300-600 \mathrm{~nm}$ spectral range of cyt $c$, Summary of CCS literature values for cyt $c$, HDX back exchange as a function of the trapping time and the incubation time with and without CIA, clustering of HDX protection groups based on the number of deuterons and the number of 
conformations, HDX back exchange as a function of the trapping time in the negative ion mode, unfolding pathway of cyt $c$, experimental results of HDX as a function of the incubation time and CCS of cyt $c$ for the +6 to +13 charge states and experimental CCS of cyt $c$ for the +14 to +21 and -5 to -10 charge states. This material is available free of charge via the Internet at http://pubs.acs.org.

\section{FUNDING SOURCES}

The authors declare no competing financial interest. The authors acknowledge the financial support from the National Institute of Health (R00GM106414), a Bruker Daltonics Inc. fellowship, and the National Science Foundation Division of Chemistry, under CAREER award CHE-1654274, with co-funding from the Division of Molecular and Cellular Biosciences to FFL.

\section{ACKNOWLEDGMENTS}

The authors would like to acknowledge the Instructional \& Research Computing Center (IRCC) at Florida International University for providing high performance computing resources that have contributed to the research results reported within this research. 
Figure 4.1 Left panel: typical mass spectra of cyt $c$ as a function of the starting solvent conditions. Right panel: overall CCS profiles (black lines) obtained by summation of the intensity-normalized IMS resolved data (color lines). The results obtained in the positive and negative ion mode are represented on the top and bottom part, respectively.
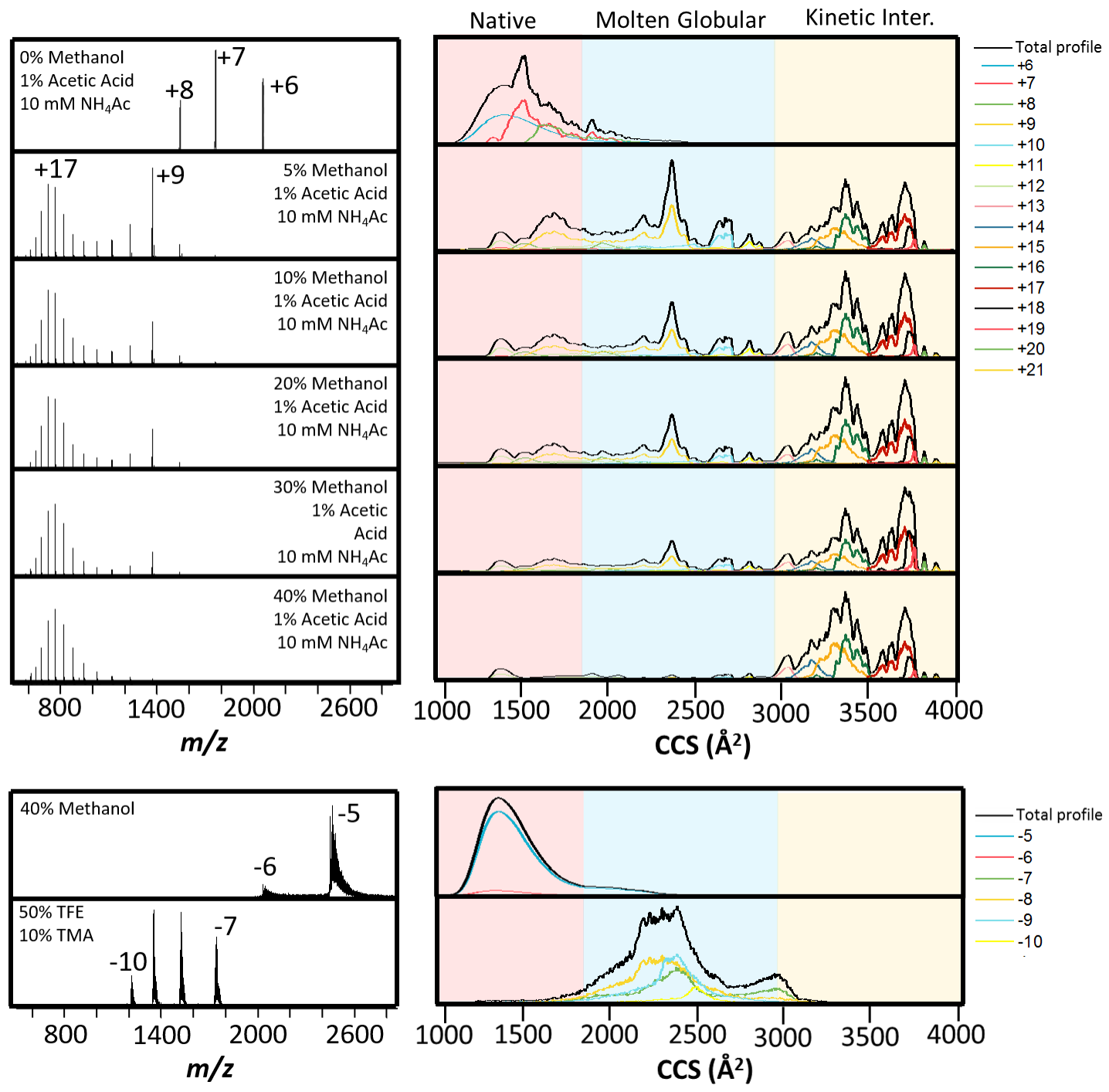
Figure 4.2 Left panel: IMS spectra of the +6 to +11 charges states of cyt $c$ as a function of the organic content (e.g., \% methanol). Right panel: IMS spectra of the +6 to +11 charge states of cyt $c$ as a function of the activation energy (e.g., deflector voltage).

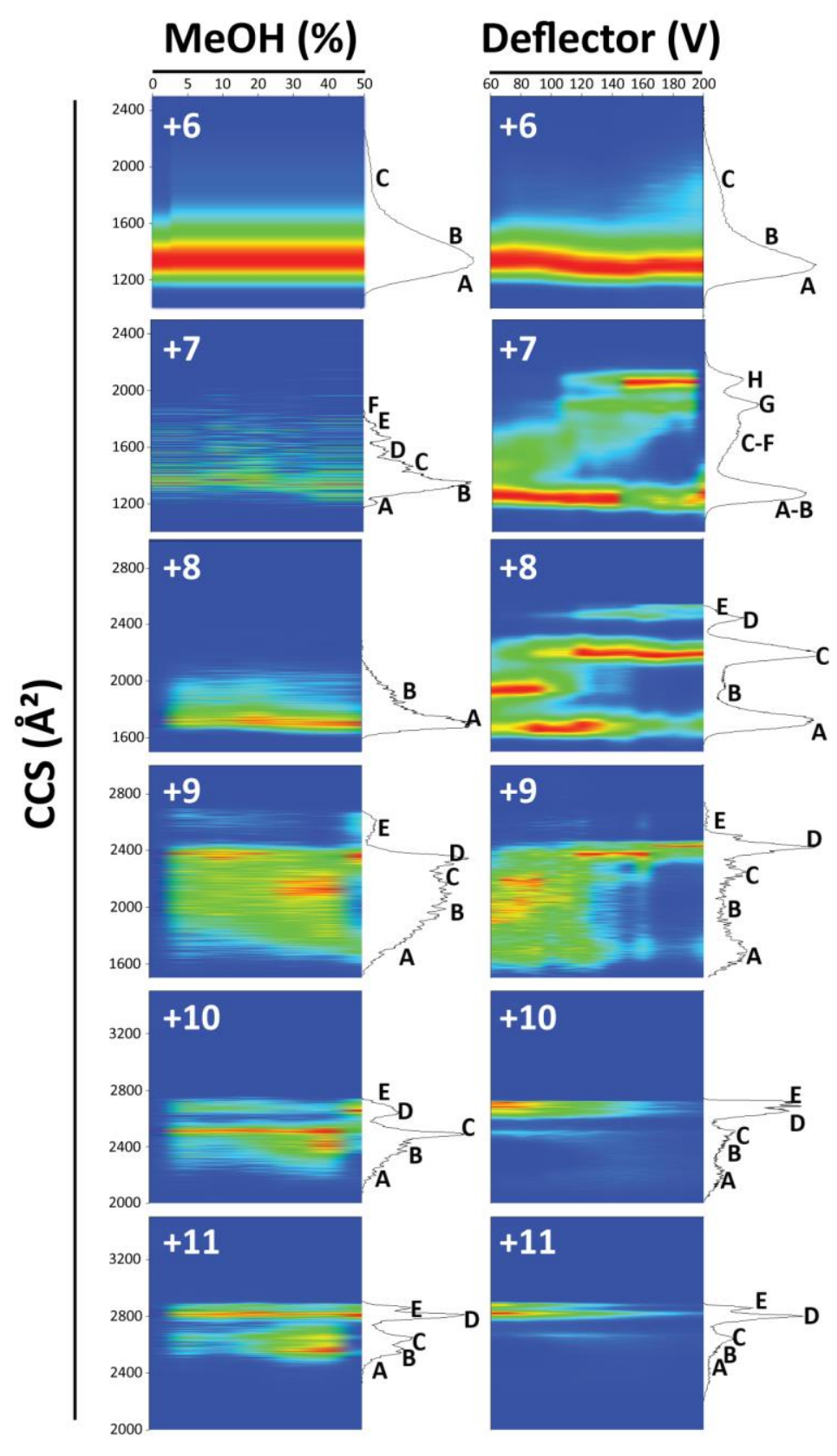


Figure 4.3 Bottom: IMS profiles obtained with CIA (blue line), and without CIA (green line). Top: High resolution IMS profiles for the +12 to +21 charge states of cyt $c$.
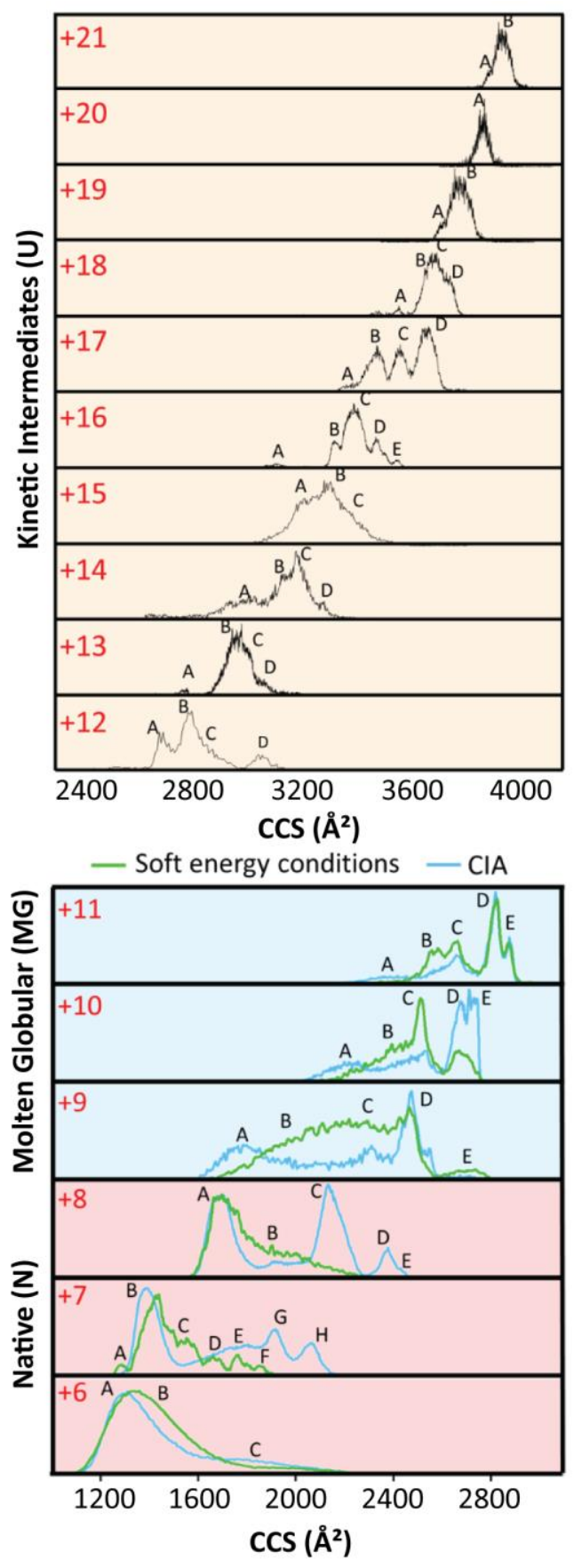
Figure 4.4 HDX back exchange as a function of the incubation time (e.g., 3 and $48 \mathrm{~h}$ ) and the time after desolvation (e.g., 0, 100, and $500 \mathrm{~ms}$ ), in terms of charge state and CCS in the positive ion mode. Clustering of the number of kinetically trapped intermediates and the initial levels of exchange allows the identification of four groups with different levels of exchange protection (color rectangles and data points).
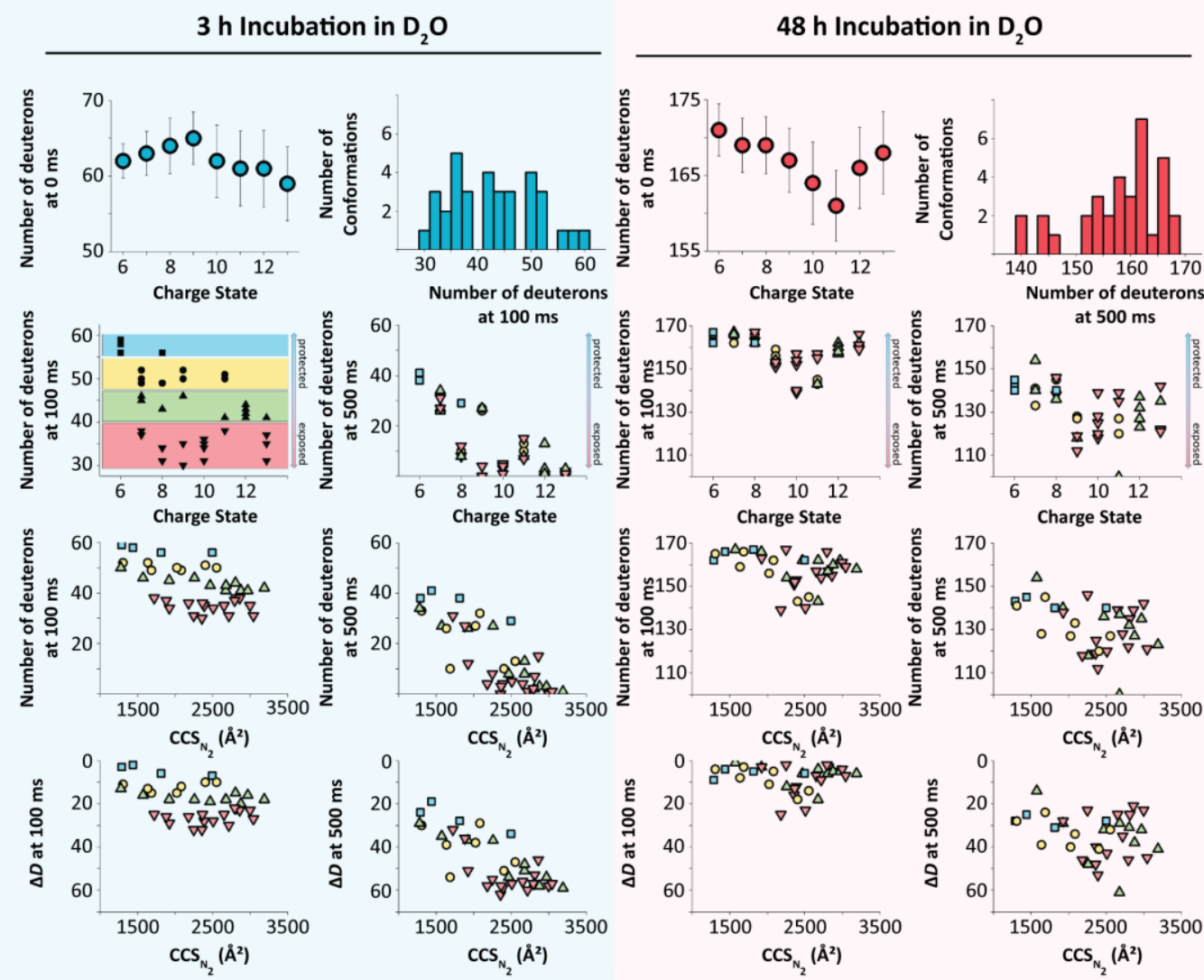
Figure 4.5 Candidate structures proposed for the kinetic intermediates IMS bands of cyt

c. Conformations $\mathrm{G}$ and $\mathrm{H}$ for the +7 charge state (dark red background); and $\mathrm{C}, \mathrm{D}$, and $\mathrm{E}$ (dark green background), for the +8 , and +9 charge states, were obtained after CIA.

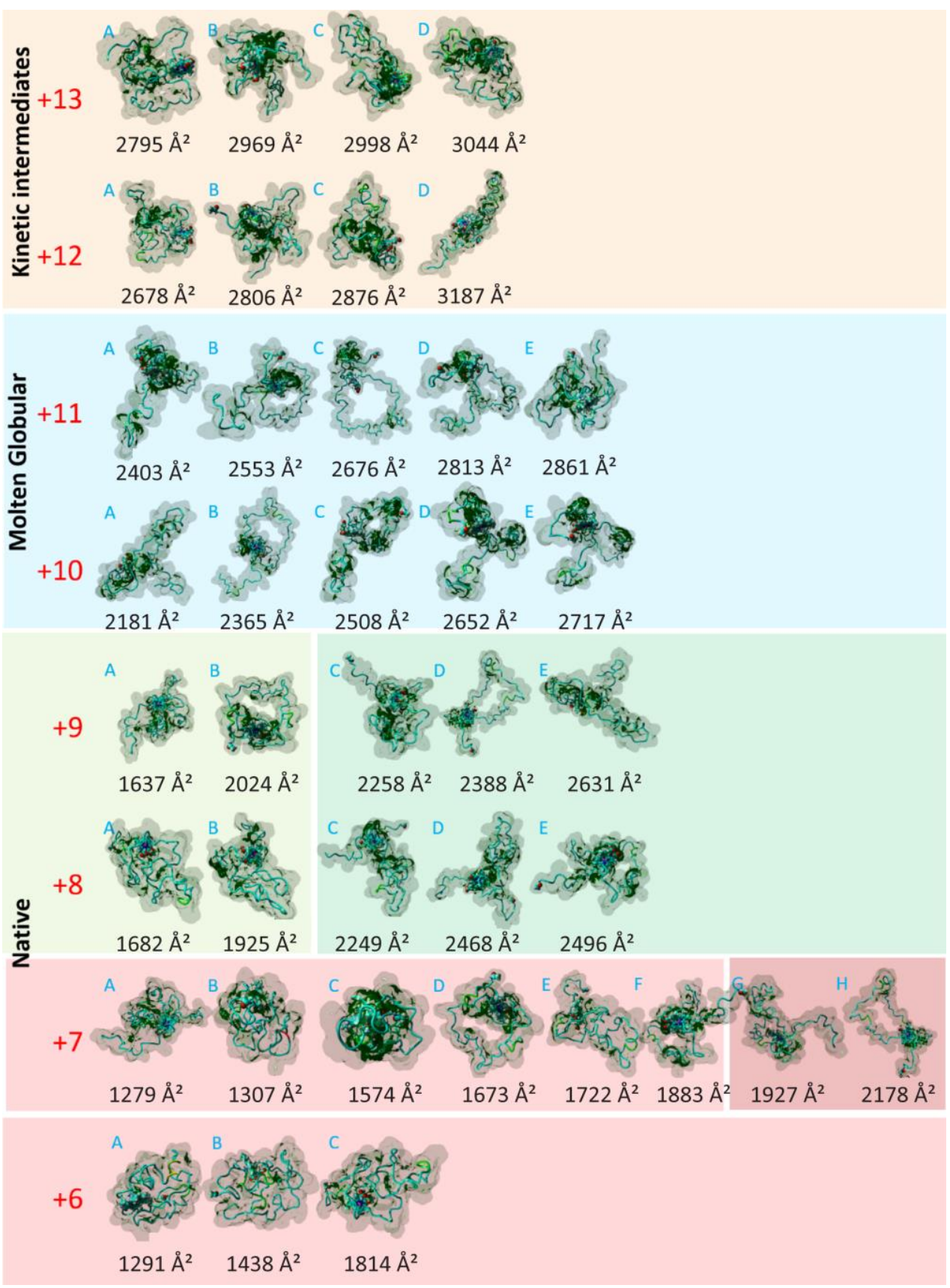




\section{LIST OF REFERENCES}

(1) Huttemann, M.; Pecina, P.; Rainbolt, M.; Sanderson, T. H.; Kagan, V. E.; Samavati, L.; Doan, J. W.; Lee, I. Mitochondrion 2011, 11, 369-381.

(2) Liu, X.; Kim, C. N.; Yang, J.; Jemmerson, R.; Wang, X. Cell 1996, 86, 147-157.

(3) Pereverzev, M. O.; Vygodina, T. V.; Konstantinov, A. A.; Skulachev, V. P. Biochem. Soc. Trans. 2003, 31, 1312-1315.

(4) Kagan, V. E.; Borisenko, G. G.; Tyurina, Y. Y.; Tyurin, V. A.; Jiang, J.; Potapovich, A. I.; Kini, V.; Amoscato, A. A.; Fujii, Y. Free. Radic. Biol. Med. 2004, 37, 1963-1985.

(5) Stevens, J. M. Metallomics : integrated biometal science 2011, 3, 319-322.

(6) Yeh, S. R.; Rousseau, D. L. Nat. Struct. Biol. 1998, 5, 222-228.

(7) Tezcan, F. A.; Findley, W. M.; Crane, B. R.; Ross, S. A.; Lyubovitsky, J. G.; Gray, H. B.; Winkler, J. R. Proc. Natl. Acad. Sci. USA 2002, 99, 8626-8630.

(8) Smith, L. J.; Kahraman, A.; Thornton, J. M. Proteins 2010, 78, 2349-2368.

(9) Sauder, J. M.; Roder, H. Fold. Des. 1998, 3, 293-301.

(10) Li, J.; Taraszka, J. A.; Counterman, A. E.; Clemmer, D. E. Int. J. Mass Spectrom. 1999, 185-187, 37-47.

(11) Wang, F.; Freitas, M. A.; Marshall, A. G.; Sykes, B. D. Int. J. Mass Spectrom. 1999, $192,319-325$.

(12) Wyttenbach, T.; von Helden, G.; Bowers, M. T. J. Am. Chem. Soc. 1996, 118, 83558364.

(13) Fernandez-Lima, F. A.; Wei, H.; Gao, Y. Q.; Russell, D. H. J. Phys. Chem. A 2009, $113,8221-8234$.

(14) Zhong, Y.; Hyung, S. J.; Ruotolo, B. T. Expert Rev. Proteomics 2012, 9, 47-58.

(15) Konermann, L.; Douglas, D. J. Biochemistry 1997, 36, 12296-12302.

(16) Shvartsburg, A. A. Anal. Chem. 2014, 86, 10608-10615.

(17) Shelimov, K. B.; Clemmer, D. E.; Hudgins, R. R.; Jarrold, M. F. J. Am. Chem. Soc. 1997, 119, 2240-2248.

(18) Bushnell, G. W.; Louie, G. V.; Brayer, G. D. J. Mol. Biol. 1990, 214, 585-595. 
(19) Suckau, D.; Shi, Y.; Beu, S. C.; Senko, M. W.; Quinn, J. P.; Wampler, F. M., 3rd; McLafferty, F. W. Proc. Natl. Acad. Sci. USA 1993, 90, 790-793.

(20) Wood, T. D.; Chorush, R. A.; Wampler, F. M., 3rd; Little, D. P.; O'Connor, P. B.; McLafferty, F. W. Proc. Natl. Acad. Sci. USA 1995, 92, 2451-2454.

(21) Valentine, S. J.; Clemmer, D. E. J. Am. Chem. Soc. 1997, 119, 3558-3566.

(22) Babu, K. R.; Moradian, A.; Douglas, D. J. J. Am. Soc. Mass Spectrom. 2001, 12, 317328.

(23) Wright, P. J.; Zhang, J.; Douglas, D. J. J. Am. Soc. Mass Spectrom. 2008, 19, 19061913.

(24) Valentine, S. J.; Clemmer, D. E. J. Am. Soc. Mass Spectrom. 2002, 13, 506-517.

(25) Campbell, S.; Rodgers, M. T.; Marzluff, E. M.; Beauchamp, J. L. J. Am. Chem. Soc. 1995, 117, 12840-12854.

(26) McLafferty, F. W.; Guan, Z. Q.; Haupts, U.; Wood, T. D.; Kelleher, N. L. J. Am. Chem. Soc. 1998, 120, 4732-4740.

(27) Fenn, J. B.; Mann, M.; Meng, C. K.; Wong, S. F.; Whitehouse, C. M. Science 1989, 246, 64-71.

(28) Clemmer, D. E.; Hudgins, R. R.; Jarrold, M. F. J. Am. Chem. Soc. 1995, 117, 1014110142.

(29) Badman, E. R.; Hoaglund-Hyzer, C. S.; Clemmer, D. E. Anal. Chem. 2001, 73, 60006007.

(30) Dickinson, E. R.; Jurneczko, E.; Pacholarz, K. J.; Clarke, D. J.; Reeves, M.; Ball, K. L.; Hupp, T.; Campopiano, D.; Nikolova, P. V.; Barran, P. E. Anal. Chem. 2015, 87, 32313238 .

(31) Fernandez-Lima, F.; Blase, R. C.; Russell, D. H. Int. J. Mass Spectrom. 2011, 298, 111-118.

(32) Fernandez-Lima, F.; Kaplan, D. A.; Suetering, J.; Park, M. A. Int. J. Ion Mobil. Spectrom. 2011, 14, 93-98.

(33) Hernandez, D. R.; Debord, J. D.; Ridgeway, M. E.; Kaplan, D. A.; Park, M. A.; Fernandez-Lima, F. Analyst 2014, 139, 1913-1921.

(34) Fernandez-Lima, F. International Journal for Ion Mobility Spectrometry 2016, 19, 6567. 
(35) Benigni, P.; Fernandez-Lima, F. Analytical Chemistry 2016, 88, 7404-7412.

(36) Schenk, E. R.; Almeida, R.; Miksovska, J.; Ridgeway, M. E.; Park, M. A.; FernandezLima, F. J. Am. Soc. Mass. Spectrom. 2015, 26, 555-563.

(37) Ridgeway, M. E.; Silveira, J. A.; Meier, J. E.; Park, M. A. Analyst 2015, 140, 69646972.

(38) Silveira, J. A.; Ridgeway, M. E.; Park, M. A. Analytical Chemistry 2014, 86, 56245627.

(39) Adams, K. J.; Montero, D.; Aga, D.; Fernandez-Lima, F. Int. J. Ion Mobil. Spectrom. 2016, 19, 69-76.

(40) Schenk, E. R.; Mendez, V.; Landrum, J. T.; Ridgeway, M. E.; Park, M. A.; FernandezLima, F. Anal. Chem. 2014, 86, 2019-2024.

(41) Schenk, E. R.; Ridgeway, M. E.; Park, M. A.; Leng, F.; Fernandez-Lima, F. Anal. Chem. 2014, 86, 1210-1214.

(42) Garabedian, A.; Butcher, D.; Lippens, J. L.; Miksovska, J.; Chapagain, P.; Fabris, D.; Ridgeway, M. E.; Park, M. A.; Fernandez-Lima, F. Physical Chemistry Chemical Physics 2016, 18, 26691-26702.

(43) Pu, Y.; Ridgeway, M. E.; Glaskin, R. S.; Park, M. A.; Costello, C. E.; Lin, C. Analytical Chemistry 2016, 88, 3440-3443.

(44) Fernandez-Lima, F. A.; Kaplan, D. A.; Park, M. A. Rev. Sci. Instrum. 2011, 82, 126106.

(45) Molano-Arevalo, J. C.; Hernandez, D. R.; Gonzalez, W. G.; Miksovska, J.; Ridgeway, M. E.; Park, M. A.; Fernandez-Lima, F. Anal Chem 2014, 86, 10223-10230.

(46) McDaniel, E. W.; Mason, E. A. Mobility and diffusion of ions in gases; John Wiley and Sons, Inc., New York: New York, 1973, p 381.

(47) United States, 1999.

(48) Schenk, E. R.; Nau, F.; Fernandez-Lima, F. Int. J. Ion Mobil. Spectrom. 2015, 18, 2329.

(49) Evans, S. V.; Brayer, G. D. J. Mol. Biol. 1990, 213, 885-897.

(50) Bogatyreva, N. S.; Ivankov, D. N. Mol. Biol. 2008, 42, 932-938.

(51) Kaltashov, I. A.; Mohimen, A. Anal. Chem. 2005, 77, 5370-5379. 
(52) Susa, A. C.; Mortensen, D. N.; Williams, E. R. J. Am. Soc. Mass Spectrom. 2014, 25, 918-927.

(53) Onufriev, A.; Case, D. A.; Bashford, D. J. Mol. Biol. 2003, 325, 555-567.

(54) Larriba, C.; Hogan, C. J., Jr. J. Phys. Chem. A 2013, 117, 3887-3901.

(55) Larriba, C.; Hogan, C. J. J. Comput. Phys. 2013, 251, 344-363.

(56) Shrivastav, V.; Nahin, M.; Hogan, C. J.; Larriba-Andaluz, C. J Am Soc Mass Spectrom 2017, 28, 1540-1551.

(57) Stellwagen, E.; Rysavy, R.; Babul, G. J. Biol. Chem. 1972, 247, 8074-8077.

(58) Stellwagen, E.; Babul, J. Biochemistry 1975, 14, 5135-5140.

(59) Drew, H. R.; Dickerson, R. E. J. Biol. Chem. 1978, 253, 8420-8427.

(60) Babul, J.; Stellwagen, E. Biochemistry 1972, 11, 1195-1200.

(61) Babul, J.; Stellwagen, E. Biopolymers 1971, 10, 2359-2361.

(62) Knapp, J. A.; Pace, C. N. Biochemistry 1974, 13, 1289-1294.

(63) Theorell, H.; Åkesson, Å. J. Am. Chem. Soc. 1941, 63, 1804-1811.

(64) Robinson, J. B., Jr.; Strottmann, J. M.; Stellwagen, E. J. Biol. Chem. 1983, 258, 67726776.

(65) Perrin, C. L. Acc. Chem. Res. 1989, 22, 268-275.

(66) Englander, S. W.; Kallenbach, N. R. Q. Rev. Biophys. 1983, 16, 521-655.

(67) Englander, S. W.; Downer, N. W.; Teitelbaum, H. Annu. Rev. Biochem. 1972, 41, 903924.

(68) Berger, A.; Loewenstein, A.; Meiboom, S. J. Am. Chem. Soc. 1959, 81, 62-67.

(69) Campbell, S.; Rodgers, M. T.; Marzluff, E. M.; Beauchamp, J. L. Journal of the American Chemical Society 1995, 117, 12840-12854.

(70) Tüchsen, E.; Woodward, C. J. Mol. Biol. 1985, 185, 405-419.

(71) Tüchsen, E.; Woodward, C. J. Mol. Biol. 1985, 185, 421-430.

(72) Lioe, H.; O'Hair, R. A.; Reid, G. E. J Am Soc Mass Spectrom 2004, 15, 65-76. 
(73) Winger, B.; Light-Wahl, K.; Rockwood, A.; Smith, R. D. Journal of the American Chemical Society 1992, 114, 5897-5898.

(74) Roder, H.; Elove, G. A.; Englander, S. W. Nature 1988, 335, 700-704.

(75) Fumo, G.; Spitzer, J. S.; Fetrow, J. S. Gene 1995, 164, 33-39.

(76) Sosnick, T. R.; Mayne, L.; Hiller, R.; Englander, S. W. Nat. Struct. Biol. 1994, 1, 149156.

(77) Hampsey, D. M.; Das, G.; Sherman, F. J. Biol. Chem. 1986, 261, 3259-3271.

(78) Lan, W.; Wang, Z.; Yang, Z.; Zhu, J.; Ying, T.; Jiang, X.; Zhang, X.; Wu, H.; Liu, M.; Tan, X.; Cao, C.; Huang, Z. X. PLoS One 2011, 6, e27219.

(79) Louie, G. V.; Hutcheon, W. L.; Brayer, G. D. J. Mol. Biol. 1988, 199, 295-314.

(80) Wu, L. C.; Laub, P. B.; Elove, G. A.; Carey, J.; Roder, H. Biochemistry 1993, 32, 10271-10276.

(81) Berghuis, A. M.; Brayer, G. D. J. Mol. Biol. 1992, 223, 959-976. 
CHAPTER 5.

CHARACTERIZATION OF THE $3{ }_{1}$ KNOT PROTEIN YIBK USING ENZYMATIC DIGESTION. MOLECULAR DYNAMICS AND TRAPPED ION MOBILITY SPECTROMETRY - MASS SPECTROMETRY 


\begin{abstract}
Knot proteins display a wide range of topologically interesting features; however, little is known about their folding pathways or the stability of the knot core. In the present work, the native state stability, intermediates and enzymatically digested knot-conserved fragments of YibK were investigated using trapped ion mobility - mass spectrometry (TIMS-MS) and molecular dynamics. Despite the high protein temperature stability under native conditions $\left(20-95^{\circ} \mathrm{C}\right)$, the conformational micro-heterogeneity and the existence of multiple unfolding intermediates were accessed using solvent denaturing agents (e.g., organic) and collisional activation. The high mobility resolving power allowed the identification of 51 mobility bands, from the native state to the unfolded state. Collisional cross sections, $\mathrm{m} / \mathrm{z}$ measurement and molecular dynamics allowed the identification of the fragment sequences (9 out of 32) that possess the 31 knot in the Yibk protein. The use of the gas-phase density of the 31 knot (CCS dependence on mass) to differentiate enzymatic fragments with a conserve knot from a collapse or unfolded fragments allows for correction of typically made mistakes during knot assignment by purely enzymatic approaches.
\end{abstract}




\section{INTRODUCTION}

Proteins with topological knots in their structure represent a new challenge for structural biology because of their untraditional folding pathways. ${ }^{1,2}$ In fact, it remains a major puzzle why these intricate structures exist and how are they formed in nature. ${ }^{3,4}$ Since their discovery, it has been shown that thousands of proteins from multiple folds and classes contain topological knots. ${ }^{5,6}$ The native state of these proteins embeds an open knot that does not disentangle completely after being pulled from both ends. ${ }^{7}$ One of these knot topologies is a $3_{1}$ knot, present in the YibK methyltransferase from Haemophilus influenza (Figure 5.1). Crystallography studies revealed that YibK adopts a structure consistent with the SpoU-type MTase, ${ }^{8}$ while a deep trefoil knot is formed at the C-terminus by the threading of the last 40 residues though a knotting loop of 39 residues. ${ }^{9}$ It has been shown that YibK can be denatured reversibly in vitro using urea, demonstrating that chaperones are not essential for the folding of the $3_{1}$ knot, while experiments using secondary and tertiary structural probes established that the protein unfolds by a mechanism involving at least one partially unfolded monomeric intermediate state. ${ }^{10-12}$ The effect of mutations in the knot core showed that the native structure remains undeveloped until very late in the folding reaction. ${ }^{13}$ It has also been suggested that a mechanism that involves an intermediate conformation with a slipknot is necessary for the folding of the $3_{1}$ knot

proteins in order to reduce the topological bottlenecks during the folding process, ${ }^{4}$ while the existence of knots in the denatured state of YibK might represent kinetically trapped states, which might be precursors to the folding pathway of the protein. ${ }^{14}$ Small-angle Xray scattering (SAXS) experiments showed that these knotted structures in the denatured 
states are different from typical random coils, while following the same random coil-like behavior. ${ }^{15}$ Computational methods suggest that the trefoil knot disappears at the end of the unfolding process, while the unfolding proceeds though four putative intermediate states. ${ }^{16,17}$ Despite the progress made on the study of $3_{1}$ knots, there is little experimental evidence on the folding intermediates and stability of 31 knots overall. In contrast to traditional structural biology tools, ion mobility spectrometry coupled to mass spectrometry (IMS-MS) has unique advantages for the study of intrinsically disordered proteins as well as systems with multiple intermediates. With the recent development of high resolution trapped IMS coupled to MS (TIMS-MS), ${ }^{18-22}$ we have shown the potential to measure collisional cross sections (CCS) and assess the micro-heterogeneity of the conformation space of biomolecules as a function of the starting solvent conditions and time after desolvation. ${ }^{23-28}$

In the present work, the thermal stability and microheterogeneity of the conformational space of the $3{ }_{1}$ knot protein YibK was investigated using high resolution TIMS-MS as a function of the starting solvent conditions (i.e., organic content and temperature) and collisional activation. The knot core was uniquely identified by enzymatic digestion using carboxypeptidase Y (CPDY), followed by TIMS-MS and molecular dynamics. In the following discussion, a special emphasis will be placed on the challenges for the identification of $33_{1}$ knot using purely enzymatic digestion, in contrast to the assessment of the $3_{1}$ knot structure using collisional cross sections, $\mathrm{m} / \mathrm{z}$ measurement and molecular dynamics of the enzymatic fragments of YibK. 


\section{MATERIALS AND METHODS}

Materials and Reagents

The YibK protein was expressed recombinantly in Escherichia coli BL21(DE3) and purified using a nickel column. A stock solution of YibK was prepared in a $10 \mathrm{mM}$ ammonium acetate $\left(\mathrm{NH}_{4} \mathrm{Ac}\right)$ buffer, dialyzed against the same buffer and diluted to a final concentration of $1 \mu \mathrm{M}$ in $10 \mathrm{mM}$ of $\mathrm{NH}_{4} \mathrm{Ac}, 95: 5,90: 10,80: 20,70: 30,60: 40$, and 50:50 (v/v) $\mathrm{H}_{2} \mathrm{O} / \mathrm{MeOH}$ prior to TIMS-MS analysis. For the enzymatic digestion, solutions with $1 \mu \mathrm{M}$ YibK in $10 \mathrm{mM} \mathrm{NH}_{4} \mathrm{Ac}$ were incubated between 1 and $18 \mathrm{~h}$ with $10 \mu \mathrm{L}$ of CPDY stock in $0.1 \mathrm{M}$ sodium acetate buffer at $26.5^{\circ} \mathrm{C}$. The reaction was quenched with the addition of $20 \mu \mathrm{L}$ of glacial acetic acid prior to TIMS-MS analysis. Carboxypeptidase Y (CPDY) was purchased from MilliporeSigma (St. Louis, MO) and diluted to a final concentration of $5 \mu \mathrm{g} / \mathrm{mL}$. Nano-ESI emitters were pulled from quartz capillaries (O.D.: $1.0 \mathrm{~mm}$ and I.D.: $0.70 \mathrm{~mm}$ ) using a P2000 laser puller (Sutter Instruments Co.). Lowconcentration Tuning Mix calibration standard (TuneMix, G24221A) was purchased from Agilent Technologies (Santa Clara, CA) and used as received. All solvents used in these studies were analytical grade or better and purchased from Fisher Scientific (Pittsburg, PA).

TIMS-MS Experiments

We employed a custom nESI-TIMS unit coupled to an Impact Q-TOF mass spectrometer (Bruker, Billerica, MA). ${ }^{29,30}$ The TIMS unit is run by custom software in LabView (National Instruments) synchronized with the MS platform controls. ${ }^{30}$ Sample aliquots (10 
$\mu \mathrm{L}$ ) were loaded in a pulled-tip capillary biased at $\sim 700-1200 \mathrm{~V}$ relative to the MS inlet. Details regarding the TIMS operation compared to traditional IMS can be found elsewhere. ${ }^{19,20,26,31,32}$ Briefly, TIMS mobility separation is based on holding the ions stationary while using an electric field against a moving buffer gas. ${ }^{33}$ The mobility, $K$, of an ion in a TIMS cell is described by:

$K=\frac{v_{g}}{E} \approx \frac{A}{\left(V_{\text {elution }}-V_{\text {out }}\right)}$

where $v_{g}, E, V_{\text {elution }}$ and $V_{\text {out }}$ are the gas velocity, applied electric field, elution voltage and base voltage, respectively. Mobility measurements were calibrated using a Tuning Mix calibration standard $\left(m / z, 322 K_{o}=1.376 \mathrm{~cm}^{2} \mathrm{~V}^{-1} \mathrm{~s}^{-1}, \mathrm{~m} / z 622 K_{o}=1.013 \mathrm{~cm}^{2} \mathrm{~V}^{-1} \mathrm{~s}^{-1}\right.$, and $\mathrm{m} / z$ $\left.922 \mathrm{~K}_{\mathrm{o}}=0.835 \mathrm{~cm}^{2} \mathrm{~V}^{-1} \mathrm{~s}^{-1}\right)$ as previously described. ${ }^{20,34}$ The same $\mathrm{rf}(880 \mathrm{kHz}$ and 280 Vpp) was applied to all electrodes including the entrance funnel, the ion mobility separating section, and the exit funnel (Figure S5.1). The TIMS separation was performed using nitrogen as a bath gas at $300 \mathrm{~K}$, and the gas flow velocity was held constant in all the experiments $\left(P_{1}=2.6\right.$ and $\left.P_{2}=1.0 \mathrm{mbar}\right)$. A total of 500 accumulations and 10 frames were acquired per TIMS experiment. Collision induced activation experiments were performed prior to the TIMS-MS by varying the electric field between the capillary outlet $\left(V_{\text {cap }}: 50-190 \mathrm{~V}\right)$, deflector plate $\left(V_{\text {def: }}: 60-200 \mathrm{~V}\right)$ and funnel entrance $\left(V_{\text {fun }}: 0-150 \mathrm{~V}\right)$ in 10V steps.

The measured mobility values $(K)$ were converted into collision cross sections (CCS, $\Omega$, $\AA^{2}$ ) using the Mason-Schamp equation: 
$\Omega=\frac{(18 \pi)^{1 / 2}}{16} \frac{z}{\left(k_{B} T\right)^{1 / 2}}\left(\frac{1}{m_{I}}+\frac{1}{m_{b}}\right)^{1 / 2} \frac{1}{K_{0}} \frac{760}{P} \frac{T}{273.15} \frac{1}{N^{*}}$

where $z$ is the charge of the ion, $\mathrm{k}_{\mathrm{B}}$ is the Boltzmann constant, $N^{*}$ is the number density and $m_{I}$ and $m_{b}$ refer to the masses of the ion and bath gas, respectively. ${ }^{33}$

Theoretical method

Briefly, molecular dynamics simulations were used to reproduce the experimental conditions and to generate the identity vectors that define YibK conformational space. The approach is similar to that previously described by Fernández-Lima et $a l .^{35}$ for peptides, with the main characteristic that the initial search targets the generation of the identity vectors, followed energy minimization. The 1MXI protein data bank entry for YibK was

utilized as the starting structure. ${ }^{9}$ All simulations were performed in a NVT thermostat using AMBER03 force field in YASARA software. The molecular dynamic simulations yielded identity vectors that cover the +7 to +12 (native) and +13 to +22 (denatured) mobility distributions, as well as models of the enzymatic fragments from CPDY digestion. The theoretical $\mathrm{CCS}_{\mathrm{N} 2}$ for each structure were calculated using the TM algorithm implemented in the iMOS software. ${ }^{36,37}$

\section{RESULTS AND DISCUSSION}

A charge state distribution dependence with the starting solution composition was observed for YibK in the positive ion mode (Figure 5.2). A bimodal distribution of charge states was observed under native-preserving solution conditions (e.g., $0 \% \mathrm{MeOH}, 10 \mathrm{mM} \mathrm{NH} 4 \mathrm{Ac}$ ), with envelopes centered at +9 (native-like state) and +12 (denatured state) charge states. 
An increase in the concentration of $\mathrm{MeOH}$ to 5\% did not show a significant change in both distributions. Conversely, the center of the denatured species envelope shifts from the +12 to the +17 charge state at $10 \% \mathrm{MeOH}$, indicating a second conformational change in the protein. Further increases in the concentration of $\mathrm{MeOH}$ decreased the relative intensity of the envelope for the lower native-like charge states (e.g., +7 to +9) and a single distribution centered at +17 was observed when the organic content was $30-50 \% \mathrm{MeOH}$.

The IMS profiles show more clearly the major conformational transitions by looking at IMS bands and relative abundances as a function of the starting solvent conditions. For example, a transition from the native-like (Figure 5.2B, green background) to the first unfolded intermediates (Figure 5.2B, blue background), and finally to the second fully unfolded species (Figure 5.2B, red background) is observed. In addition, other less pronounced conformational transitions can be distinguished.

In addition to the starting solvent dependence, the conformational space microheterogeneity of the 31 knot YibK protein was studied using collisional induced activation (Figures 5.3, S5.2 and S5.3). Examination of the mobility profiles showed that native-like ions with $\mathrm{CCS}_{\mathrm{N} 2} \sim 2200-2500 \AA^{2}$ are generated during the nanoESI process for the +7 to +11 charge states, followed by a structural transition where the knot core is undone, and finally, a fully open conformation with $\mathrm{CCS}_{\mathrm{N} 2} \sim 5600 \AA^{2}$ for the +22 charge state. The IMS profiles generated from starting solutions with 0 and $10 \% \mathrm{MeOH}$ (e.g., Figure 5.2, blue, yellow, and green profiles) showed that the transition intermediates are generated for the +10 charge state and forward. However, the solvation effect of the higher amount of organic content when the starting solution contains $50 \% \mathrm{MeOH}$ caused a rapid, 
cooperative unfolding towards the unknotted conformation, observed as early as in the +8 charge state. It is noteworthy that following the disrupture of the knot, the additional collisional energy can trigger a structural collapse (e.g., Figure 5.2b, green profiles and yellow profiles). We interpret this result as a consequence of the unfolding of the knot core, driven by the added internal energy, followed by the rearrangement of the protein backbone into a compact conformation with a local minima in the free energy landscape. Under the CIA regime, the fully denatured conformation with little contribution from the unfolding intermediates is observed at the +15 charge state.

The $3_{1}$ knot YibK protein conformational space was studied as a function of the charge state varying the staring solution and CIA conditions (Figures S5.2 and S5.3). Examination of the TIMS countor plots (Figures S5.2 and S5.3) showed that an open intermediate is observed for most charge states (e.g., +8 to +17 ) when the amount of organic content increases in the starting solution. This result suggests that a cooperative unfolding is favored by the $\mathrm{MeOH}$ in solution (Figures S5.2). In contrast to the starting solution conditions experiments, the examination of the contour plots generated as a function of the CIA energy also showed that a structural collapse takes place with the collisional energy increased. The contour plots for the +7 to +10 charge states exhibited little variation when the activation energy is increased (Figures S5.3), as the energy used to activate the ions is not enough to disrupt the intramolecular interactions from the hydrogen bond network that holds together the more compact, native-like knotted conformations. The contour plots for the +18 to +22 showed that the kinetic intermediates are already unfolded, and the additional energy does leads to more extended conformations. 
Theoretical $\mathrm{CCS}_{\mathrm{N} 2}$ were calculated for the native and the unfolded structures of YibK using IMoS (Figure 5.2A, blue and red triangles). It is important to note that the TM algorithm implemented on IMoS can measure the $\mathrm{CCS}_{\mathrm{N} 2}$ for different charge states on the same structure, using the location and accessibility of basic sites where protonation is possible. The results of these calculations were in good agreement with the experimental results obtained with TIMS for the low (i.e., +7 to +9 ) and high (i.e., +19 to +22 ) charge states, while a pronounced deviation is evident after the +10 charge state. The residues that make up the knot core of YibK is shown in red in the candidate structures shown on Figure 5.2.

Solutions in 0,10 , and $50 \% \mathrm{MeOH}$ were incubated at $20,35,50,65,80$, and $95^{\circ} \mathrm{C}$ for $1 \mathrm{~h}$ to study the thermal stability of the knotted structure of YibK (Figure 5.3). As in Figure 5.1, the overall $\mathrm{CCS}_{\mathrm{N} 2}$ profiles (Figure 5.3, black lines) were obtained by summation of the ion mobility resolved data (Figure 5.3, color lines), where a predominantly population of native-like conformations is observed at all temperature values when the starting solution does not contain any amount of organic content (Figure 5.3A). In contrast, when the solution had 10 and $50 \% \mathrm{MeOH}$, the population of ions observed was mostly unfolded, while a mixture of folded/knotted, native-like species (i.e., lower charge states) was also observed in lesser abundance (Figures 5.3B and 5.3C). Remarkably, the effect of temperature in the overall mobility distribution is not very noticeable, as only a slight narrowing of the profiles was observed when the temperature increased. These results suggest that the stability of the knot core is more susceptible to structural changes that result the solvation effects of the organic molecules than the effects of the additional energy provided by the increasing temperature in the starting solution. The narrowing of the 
mobility bands can be explained as the overall flexibility of the trapped kinetic intermediates is decreased to achieve a stable conformation and counteract the increasing energy provided by the surrounding solvent molecules in solution.

This discussion can be followed by the study of the stability and integrity of the knot core. The region is defined as the shortest sub-chain within the protein for which a knot is stable. So far, the residues in the core are predicted using algorithms described by the mathematical field of knot theory, which studies the entanglement in closed chains, but has been extended to characterize knotting in open polypeptide chains. ${ }^{38,39}$ It is worth mentioning that to date, no experimental methodologies have been reported to interrogate the integrity of the core without the stabilizing interactions provided by the residues that belong to the $\mathrm{C}$ - and $\mathrm{N}$-termini, or to predict the size (i.e., CCS) of fragments that embed an intact core (i.e., not unfolded nor collapsed). By investigating the digestion products of YibK using IMS, we can accurately determine which fragments are retaining the native conformation and integrity of the knot core. The high resolution of TIMS-MS permits to concomitantly analyze all the digestion products of YibK in terms of their size. Then, it is possible to identify which fragments unfold, collapse, or retain the integrity of the core by comparing between the experimental and theoretical $\mathrm{CCS}_{\mathrm{N} 2}$ of the models generated by MD for each one of the observed fragments. The trefoil $3_{1}$ knot core found in the recombinant YibK is 43 amino acids long, between residues 91 and 134 (Figure 5.4B, blue region). An enzymatic digestion of YibK using CPDY was performed to isolate the core from the knot tails (Figure 5.4 and Table S5.1). TIMS-MS analysis of the digestion showed that after $18 \mathrm{~h}$ of incubation, the reaction yielded 32 fragments with a maximum of 74 
residues missing from the $\mathrm{C}$-terminal, 53 from the $\mathrm{N}$-terminal, and 128 from both termini concomitantly (Figure 5.4B). Our results exhibited that the fragments containing a knot in their structure had a larger size than globular proteins of similar mass. This was evident when comparing the reported CCS of globular proteins (Figure 5.4A, blue trendline) with the CCS of the digestion fragments of YibK. Moreover, the fragments that lost the integrity provided by the knot core, collapsed while a rearrangement of their overall structure adopts a conformation smaller than expected if the protein was globular (Figure 5.4A, black circles). Among the 32 fragments, 9 conserved the knot integrity in their structure (Figures 5.4A, black stars and 5.4B). Only the native charge states of the full sequence (i.e., +7 to +9) displayed a unique conformation, while the rest of the fragments unfolded and collapsed evidenced by multiple conformations (Figure 5.4C). All the fragments containing the knot core in their structure display a trend (Figure 5.4A, grey line), while is considered that the structures with CCS above this threshold unfold due the loss of the knot core.

\section{CONCLUSIONS}

Our work provides, for the first time, insight into the unique structural dynamics and conformational landscape of the knotted protein YibK in the gas-phase. The high ion mobility resolving power of the TIMS analyzer allowed the identification of detailed mobility bands between the +7 to +22 charge states as a function of the starting solution conditions and the activation energy regime implemented. The experimental results showed that, under native preserving solution and energy conditions, a bimodal distribution of charge states for the native/knotted and denatured/unfolding is observed, while a cooperative unfolding is favored by the solvation effect of the organic content in solution. 
Overlapping of the IMS bands for the observed charge states under different solution/energy conditions revealed that the knotting/folding pathway of YibK follows, at least, four conformational trends. The calculated $\mathrm{CCS}_{\mathrm{N} 2}$ of molecular models for the native and denatured forms of YibK is consistent with the experimentally measured $\mathrm{CCS}_{\mathrm{N} 2}$. The temperature dependence of the knotted conformation of YibK was also examined, revealing that the tertiary structure is stable between $20-95^{\circ} \mathrm{C}$. However, the solvation effect of as little as $10 \% \mathrm{MeOH}$ is enough to cause a major a disruption of the intramolecular interactions that hold this intricate conformation together. Nevertheless, the increasing temperature did not change the relative population of the higher charge states when $\mathrm{MeOH}$ was present in the starting solution. Finally, the stability and integrity of the knot core was also studied. Removal of the knot tails by enzymatic digestion with CPDY allowed the analysis of charged fragments that contained the residues that makeup the knot core. The experimentally measured $\mathrm{CCS}_{\mathrm{N} 2}$ of each fragment was compared with theoretically calculated $\mathrm{CCS}_{\mathrm{N} 2}$ of models that matched the amino acid length of each fragment. A total of 32 fragments were observed, while the knot was found to be intact in 9 of them. Our results suggest that the loss of the knot core can either cause a structural collapse or an unfolding of protein. The collapsed structures are found to be smaller than globular proteins of the similar mass.

The analytical workflow implemented in this work describes powerful TIMS-based strategies for the analysis of the complex conformations found in many biomolecules. Empirical information was obtained by modifying the conditions of the starting solutions, the activation energy regime, the thermal stability of the protein conformation, and the 
isolation of the structural regions of interest by enzymatic digestion. Combined with molecular dynamics and algorithms that calculate the $\mathrm{CCS}_{\mathrm{N} 2}$ of molecular models, is possible to obtain important information on the conformational landscapes of these rare topologies.

\section{ASSOCIATED CONTENT}

Scheme of the TIMS cell, contour plots of each charge state as a function of the staring solution conditions and CIA energy and summary of the information on each fragment obtained after digestion with CPDY.

\section{FUNDING SOURCES}

The authors declare no competing financial interest. The authors acknowledge the financial support from the National Institute of Health (R00GM106414), a Bruker Daltonics Inc. fellowship, and the National Science Foundation Division of Chemistry, under CAREER award CHE-1654274, with co-funding from the Division of Molecular and Cellular Biosciences to FFL.

\section{ACKNOWLEDGMENTS}

The authors would like to acknowledge the Instructional \& Research Computing Center (IRCC) at Florida International University for providing high performance computing resources that have contributed to the research results reported within this research. 
Figure 5.1 Typical mass spectra of YibK in the positive mode as a function of the starting solvent conditions. Overall $\mathrm{CCS}_{\mathrm{N} 2}$ profiles (black lines) obtained by summation of the intensity-normalized IMS resolved data (color lines). Green background, native-like states; blue background: transition states; red background: unfolded states.
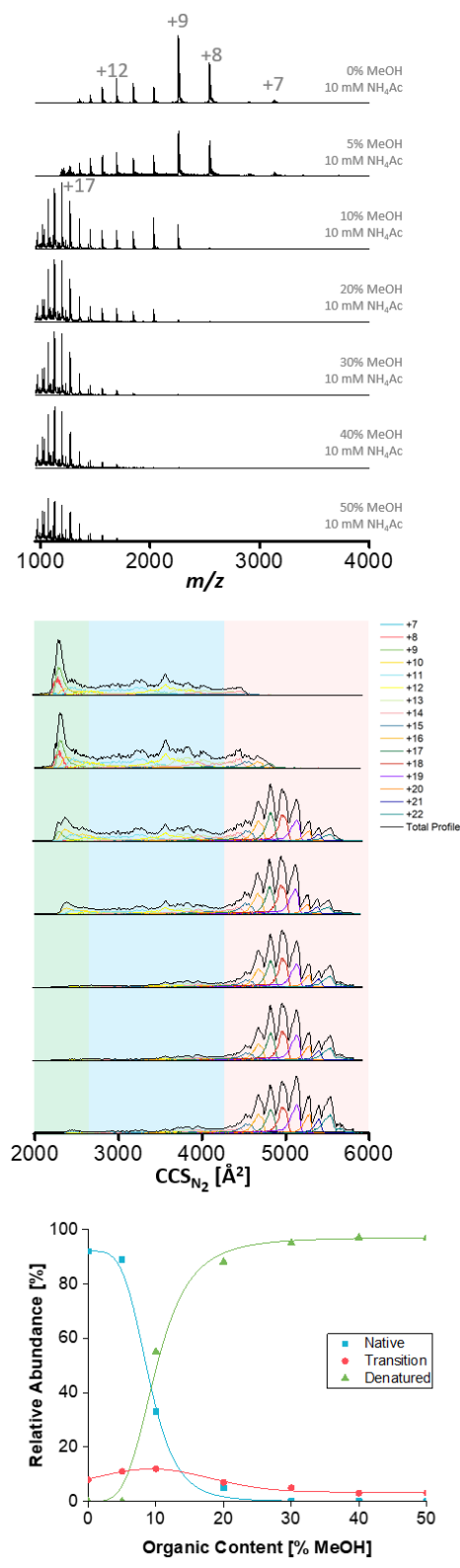
Figure 5.2 IMS profiles of the +7 to +22 charge states of YibK as a function of the organic content (e.g., \% MeOH) in the starting solution. Molecular representations and theoretical $\mathrm{CCS}_{\mathrm{N} 2}$ of the native/knotted (blue triangles) and denatured/unfolded (red triangles) of YibK are shown. IMS profiles of the +8 to +22 charge states of YibK as a function of the CIA regime (e.g., on/off) for a solution with $10 \% \mathrm{MeOH}$.

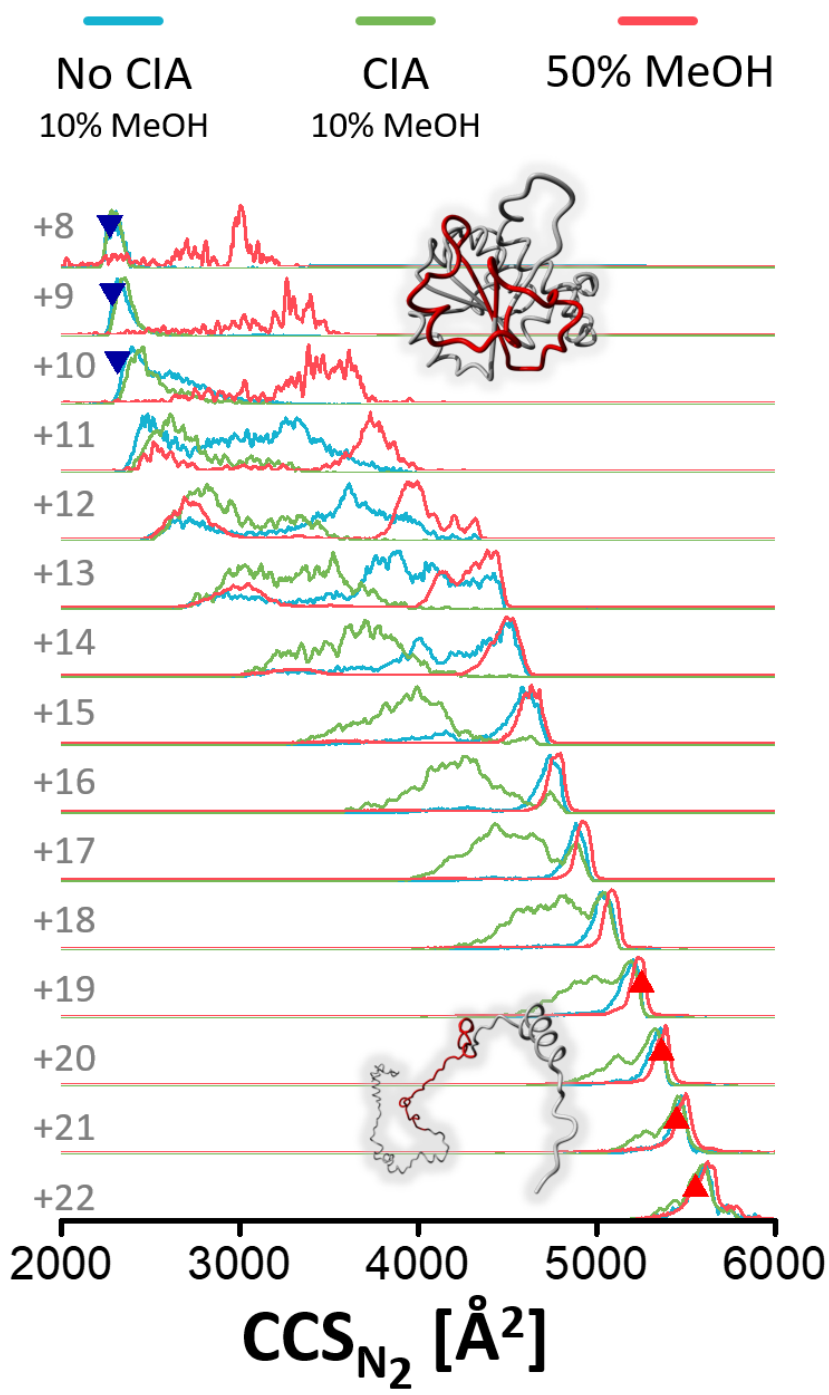


Figure 5.3 Overall $\mathrm{CCS}_{\mathrm{N} 2}$ profiles (black lines) obtained by summation of the intensitynormalized IMS resolved data (color lines) obtained as a function of the starting solution temperature.
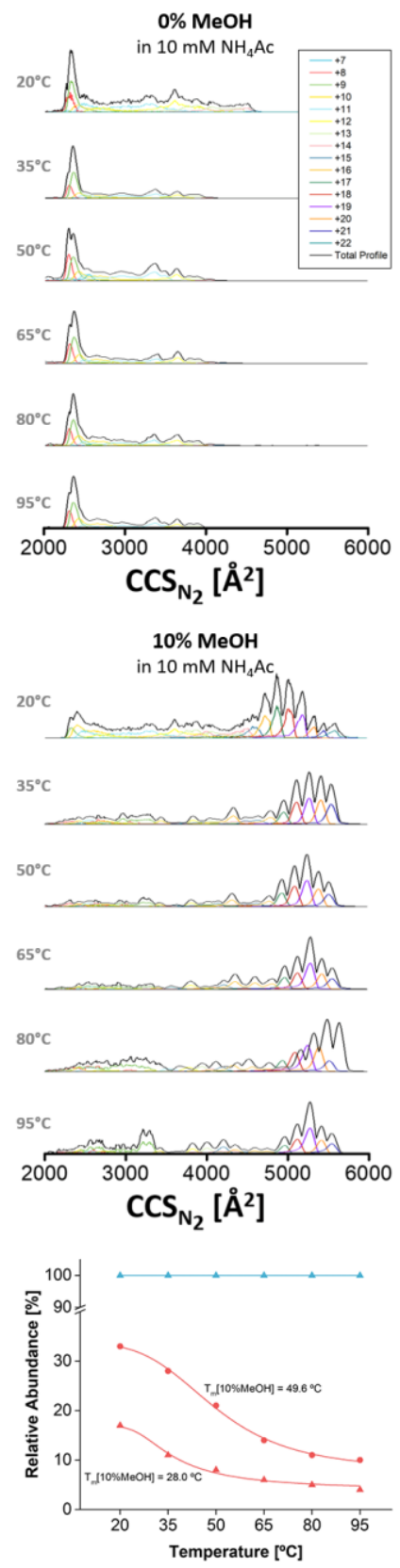
Figure 5.4 Digestion of YibK with CPDY. A: $\mathrm{CCS}_{\mathrm{N} 2}$ distribution of the observed fragments as a function of the mass of each fragment. Orange circles: undigested protein; Green triangles: fragments with residues removed exclusively from the C-terminal; Red triangles: fragments with residues removed exclusively from the N-terminal; Blue rhomboids: fragments with residues removed from the both termini; Black circles: fragments where a structural collapse is observed; Black stars and grey line: fragments where the knot is preserved after the digestion; Blue line: distribution of reported $\mathrm{CCS}_{\mathrm{N} 2}$ for globular proteins. B: Sequence coverage by the digestion. C: IMS profiles of the fragments that preserve the knot.

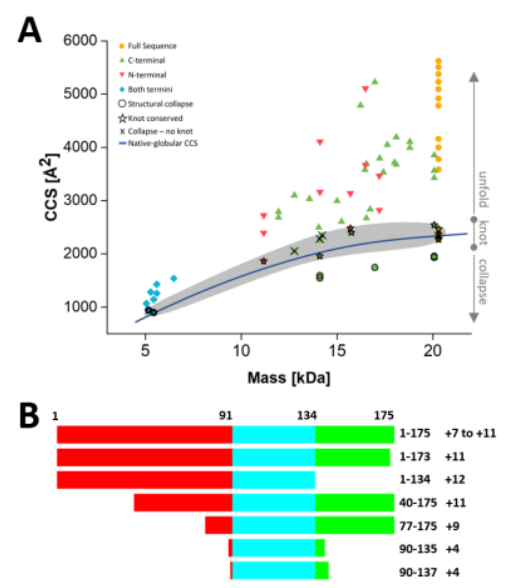

C

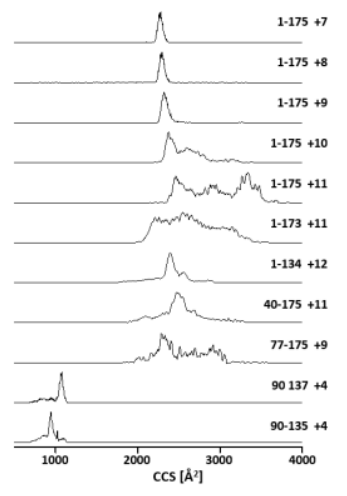




\section{LIST OF REFERENCES}

(1) Andersson, F. I.; Pina, D. G.; Mallam, A. L.; Blaser, G.; Jackson, S. E. FEBS J 2009, 276, 2625-2635.

(2) Mallam, A. L. FEBS J 2009, 276, 365-375.

(3) Yeates, T. O.; Norcross, T. S.; King, N. P. Current opinion in chemical biology 2007, $11,595-603$.

(4) Sulkowska, J. I.; Sulkowski, P.; Onuchic, J. Proc Natl Acad Sci U S A 2009, 106, 3119 3124.

(5) Virnau, P.; Mirny, L. A.; Kardar, M. PLoS Comput Biol 2006, 2, e122.

(6) Taylor, W. R. Computational biology and chemistry 2007, 31, 151-162.

(7) Sułkowska, J. I.; Sułkowski, P.; Szymczak, P.; Cieplak, M. 2010.

(8) Anantharaman, V.; Koonin, E. V.; Aravind, L. Journal of molecular microbiology and biotechnology 2002, 4, 71-75.

(9) Lim, K.; Zhang, H.; Tempczyk, A.; Krajewski, W.; Bonander, N.; Toedt, J.; Howard, A.; Eisenstein, E.; Herzberg, O. Proteins 2003, 51, 56-67.

(10) Mallam, A. L.; Jackson, S. E. J Mol Biol 2005, 346, 1409-1421.

(11) Mallam, A. L.; Jackson, S. E. J Mol Biol 2007, 366, 650-665.

(12) Mallam, A. L.; Jackson, S. E. J Mol Biol 2006, 359, 1420-1436.

(13) Mallam, A. L.; Morris, E. R.; Jackson, S. E. Proc Natl Acad Sci U S A 2008, 105, 18740-18745.

(14) Mallam, A. L.; Rogers, J. M.; Jackson, S. E. Proc Natl Acad Sci U S A 2010, 107, 8189-8194.

(15) Shih, P. M.; Wang, I.; Lee, Y. T.; Hsieh, S. J.; Chen, S. Y.; Wang, L. W.; Huang, C. T.; Chien, C. T.; Chang, C. Y.; Hsu, S. T. J Phys Chem B 2015, 119, 5437-5443.

(16) Mallam, A. L.; Onuoha, S. C.; Grossmann, J. G.; Jackson, S. E. Mol Cell 2008, 30, 642-648.

(17) Tuszynska, I.; Bujnicki, J. M. Journal of biomolecular structure \& dynamics 2010, 27, 511-520. 
(18) Fernandez-Lima, F.; Blase, R. C.; Russell, D. H. Int. J. Mass Spectrom. 2011, 298, 111-118.

(19) Fernandez-Lima, F.; Kaplan, D. A.; Suetering, J.; Park, M. A. Int. J. Ion Mobil. Spectrom. 2011, 14, 93-98.

(20) Hernandez, D. R.; Debord, J. D.; Ridgeway, M. E.; Kaplan, D. A.; Park, M. A.; Fernandez-Lima, F. The Analyst 2014, 139, 1913-1921.

(21) Fernandez-Lima, F. International Journal for Ion Mobility Spectrometry 2016, 19, 6567.

(22) Benigni, P.; Fernandez-Lima, F. Analytical Chemistry 2016, 88, 7404-7412.

(23) Molano-Arevalo, J. C.; Jeanne Dit Fouque, K.; Pham, K.; Miksovska, J.; Ridgeway, M. E.; Park, M. A.; Fernandez-Lima, F. Anal Chem 2017, 89, 8757-8765.

(24) Molano-Arevalo, J. C.; Gonzalez, W.; Jeanne dit fouque, K.; Miksovska, J.; Maitre, P.; Fernandez-Lima, F. Physical Chemistry Chemical Physics 2018.

(25) Schenk, E. R.; Mendez, V.; Landrum, J. T.; Ridgeway, M. E.; Park, M. A.; FernandezLima, F. Anal. Chem. 2014, 86, 2019-2024.

(26) Schenk, E. R.; Ridgeway, M. E.; Park, M. A.; Leng, F.; Fernandez-Lima, F. Anal. Chem. 2014, 86, 1210-1214.

(27) Garabedian, A.; Butcher, D.; Lippens, J. L.; Miksovska, J.; Chapagain, P.; Fabris, D.; Ridgeway, M. E.; Park, M. A.; Fernandez-Lima, F. Physical Chemistry Chemical Physics 2016, 18, 26691-26702.

(28) Pu, Y.; Ridgeway, M. E.; Glaskin, R. S.; Park, M. A.; Costello, C. E.; Lin, C. Analytical Chemistry 2016, 88, 3440-3443.

(29) Fernandez-Lima, F.; Kaplan, D. A.; Suetering, J.; Park, M. A. International journal for ion mobility spectrometry : official publication of the International Society for Ion Mobility Spectrometry 2011, 14, 93-98.

(30) Fernandez-Lima, F. A.; Kaplan, D. A.; Park, M. A. Rev Sci Instrum 2011, 82, 126106.

(31) Fernandez-Lima, F. A.; Kaplan, D. A.; Park, M. A. Rev. Sci. Instrum. 2011, 82, 126106.

(32) Molano-Arevalo, J. C.; Hernandez, D. R.; Gonzalez, W. G.; Miksovska, J.; Ridgeway, M. E.; Park, M. A.; Fernandez-Lima, F. Anal. Chem. 2014, 86, 10223-10230.

(33) McDaniel, E. W.; Mason, E. A. Mobility and diffusion of ions in gases; John Wiley and Sons, Inc., New York: New York, 1973, p 381. 
(34) United States, 1999.

(35) Fernandez-Lima, F. A.; Wei, H.; Gao, Y. Q.; Russell, D. H. J. Phys. Chem. A 2009, $113,8221-8234$.

(36) Larriba, C.; Hogan, C. J., Jr. J. Phys. Chem. A 2013, 117, 3887-3901.

(37) Larriba, C.; Hogan, C. J. J. Comput. Phys. 2013, 251, 344-363.

(38) Taylor, W. R. Nature 2000, 406, 916-919.

(39) Sulkowska, J. I.; Rawdon, E. J.; Millett, K. C.; Onuchic, J. N.; Stasiak, A. Proc Natl Acad Sci U S A 2012, 109, E1715-1723. 
CHAPTER 6.

INSIGHTS FROM TRAPPED ION MOBILITY SPECTROMETRY - MASS

SPECTROMETRY ON THE STRUCTURAL INTEGRITY OF THE KNOT PROTEIN UBIQUITIN C-TERMINAL HYDROLASE 


\begin{abstract}
Knotted proteins are fascinating proteins whose backbones adopt knotted configurations with a wide range of topologically interesting features. In the present work, we are providing, for the first time, experimental insights on the structural integrity of the kinetically trapped intermediates of the knot protein ubiquitin C-terminal hydrolase (UCH), investigated using trapped ion mobility spectrometry - mass spectrometry (TIMSMS) as a function of the starting solvent conditions (i.e., organic content), collision induced activation (CIA), and starting solution temperature. The approach implemented in the present study allowed the simultaneous interrogation of the kinetically trapped intermediates that are part of the conformational space of the knot proteins. Our results suggest that the $5_{2}$ knot present in $\mathrm{UCH}$ provides an increased stability that protects the tertiary structure of the protein against denaturing agents (i.e., high organic content, increased activation energy, and increased temperature). In addition, the stability of the knot core was evaluated by enzymatic digestion of the knot tails. Our results suggest that the loss of the knot core can cause a structural collapse, as the knotted structure occupies a larger space than a globular structure of similar mass. The potential of the present approach is demonstrated to provide an invaluable insight necessary for the structural integrity elucidation of the knot proteins in the gas-phase.
\end{abstract}




\section{INTRODUCTION}

Understanding the mechanisms of protein folding is critical as they underpin virtually all cellular processes. Our knowledge of these structural details is inevitably entwined to the analytical methodologies available, and their ability in overcoming the complexity of these macromolecules. There is a growing interest in developing 'hybrid' strategies which combine the benefits of different technologies to characterize the most challenging protein structures. ${ }^{1}$ One way to study the mechanisms of protein folding is to analyze protein chains through constricted spaces. This approach offers potentially valuable model systems for conducting theoretical and experimental studies of protein dynamics. ${ }^{2}$ However, conventional techniques cannot detect folding intermediates efficiently, and usually only the most stable structure for a given set of conditions is observed. ${ }^{3,4}$

Knotted proteins are fascinating proteins whose backbones adopt knotted configurations with a wide range of topologically interesting features, which are clarified by looking more carefully at issues concerning the termini of the proteins. ${ }^{5}$ Even though the structure of many proteins has been elucidated, many embedded knots were overlooked as visual inspection is hard, requiring a computational approach. ${ }^{6}$ However, various knot types have been identified in proteins as current research on these structures has shown that thousands of proteins from multiple folds and classes are known to contain knots: trefoil (knot designation $3_{1}$ ), figure eight knot $\left(4_{1}\right), 5_{2}$ knot, and 6 knot. $^{7-9}$ Moreover, it is still not clear

why these intricate structures exist in nature or how their folding takes place. ${ }^{5,10}$. When analyzing knotted proteins, questions arise immediately about how such proteins can fold efficiently. ${ }^{11-14}$ 
One of the most complex knots found in proteins so far is the $5_{2}$ knot in the human enzyme ubiquitin C-terminal hydrolase (UCH). The knot core of $\mathrm{UCH}$ embeds five distinct crossings of its polypeptide chain, and is one of the two knotted structures identified in humans so far, along with carbonic anhydrase. ${ }^{8,14} \mathrm{UCH}$ is a $26 \mathrm{kDa}$ cysteine protease that plays an important role in the ubiquitin-proteasome system. ${ }^{15,16}$ Until now, only one study on the folding of UCH has been carried out. ${ }^{14}$ This study examined the unfolding of UCH under equilibrium conditions using chemical denaturants and both fluorescence and farUV CD to probe secondary and tertiary structures. The results of the study showed that UCH unfolds reversibly in vitro without the help of molecular chaperones. They also showed that several phases of folding and unfolding reactions are observed, while the folding mechanism involves two hyperfluorescent intermediates.

The nascent area of research on knotted proteins will require new experimental approaches in order to provide conclusive answers about the roles of knots in proteins. Ion mobility spectrometry coupled to mass spectrometry (IMS-MS) can provide sufficient information for the elucidation of folding dynamics of proteins with knotted backbones. It is worth mentioning that to date, no published method regarding the combined use of IMS-MS and computational dynamics approaches for the determination of the folding landscape of knotted proteins. Trapped IMS-MS(TIMS-MS), ${ }^{17-21}$ and more recently complemented with collision-induced activation (CIA), ${ }^{22}$ allows the simultaneous mobility measurement of multiple trapped kinetic intermediates. ${ }^{23-26}$ This study combines the structure elucidation capabilities of TIMS-MS to explain the conformational dynamics of the knot protein UCH, while providing unique insights into the folding pathways and stabilization mechanisms. 
Our results showed the potential of the present approach to provide an invaluable insight necessary for the structural elucidation of these intricate knotted topologies in the gasphase. In the following discussion, a special emphasis will be placed on the structural integrity and stability of the knot present in $\mathrm{UCH}$.

\section{MATERIALS AND METHODS}

Materials and Reagents

All solvents used were analytical grade or better and purchased from Fisher Scientific (Pittsburg, PA). UCH was expressed recombinantly in Escherichia coli BL21(DE3) and purified using a nickel column. The protein was shown to be pure using both SDS/PAGE and MS analysis. A stock solution of UCH was prepared in a buffer of $10 \mathrm{mM}$ ammonium acetate $\left(\mathrm{NH}_{4} \mathrm{Ac}\right)$, dialyzed against the same buffer and diluted to a final concentration of $10 \mu \mathrm{M}$ in10 mM of $\mathrm{NH}_{4} \mathrm{Ac}, 95: 5,90: 10,80: 20,70: 30,60: 40$, and 50:50 (v/v) $\mathrm{H}_{2} \mathrm{O} / \mathrm{MeOH}$. Nano-ESI emitters were pulled from quartz capillaries (O.D.: $1.0 \mathrm{~mm}$ and I.D.: $0.70 \mathrm{~mm}$ ) using Sutter Instruments Co. P2000 laser puller. Low-concentration Tuning Mix calibration standard (TuneMix, G24221A) was purchased from Agilent Technologies (Santa Clara, CA). A stock solution of carboxypeptidase y (CPDY) was prepared with a final concentration of $1 \mathrm{mg} / \mathrm{mL}$. Prior incubation, $2 \mu \mathrm{L}$ of CPDY were added to a solution of $200 \mu \mathrm{L}$ of $\mathrm{UCH}$. Incubation was performed at $26.5^{\circ} \mathrm{C}$ for $18 \mathrm{~h}$. When the incubation was finished, the enzymatic reaction was quenched by the addition of $20 \mu \mathrm{L}$ of glacial acetic acid. 


\section{CIA-TIMS-MS Experiments}

We employed a custom nESI-TIMS unit coupled to an Impact Q-TOF mass spectrometer (Bruker, Billerica, MA). ${ }^{27,28}$ The TIMS unit is run by custom software in LabView (National Instruments) synchronized with the MS platform controls. ${ }^{28}$ Sample aliquots (10 $\mu \mathrm{L})$ were loaded in a pulled-tip capillary biased at $\sim 1000 \mathrm{~V}$ to the MS inlet. Details regarding the TIMS operation compared to traditional IMS can be found elsewhere. ${ }^{18,19,24,29,30}$ Briefly, TIMS mobility separation is based on holding the ions stationary using an electric field against a moving buffer gas. ${ }^{31}$ The mobility, $K$, of an ion in a TIMS cell is described by:

$K=\frac{v_{g}}{E}=\frac{A}{\left(V_{\text {elution }}-V_{\text {out }}\right)}$

where $v_{g}, E, V_{\text {elution }}$ and $V_{\text {out }}$ are the gas velocity, applied electric field, elution voltage and base voltage, respectively. The constant $A$ was determined using a Tuning Mix calibration standards $\left(m / z, 322 K_{o}=1.376 \mathrm{~cm}^{2} \mathrm{~V}^{-1} \mathrm{~s}^{-1}, m / z 622 K_{o}=1.013 \mathrm{~cm}^{2} \mathrm{~V}^{-1} \mathrm{~s}^{-1}\right.$, and $m / z 922 \mathrm{~K}_{o}=$ $\left.0.835 \mathrm{~cm}^{2} \mathrm{~V}^{-1} \mathrm{~s}^{-1}\right) \cdot{ }^{19,32}$

The same rf ( $880 \mathrm{kHz}$ and $280 \mathrm{Vpp}$ ) was applied to all electrodes including the entrance funnel, the ion mobility separating section, and the exit funnel (Figure S6.1). TIMS separation was performed using nitrogen as a bath gas at $300 \mathrm{~K}$, and the gas flow velocity was held constant in all the experiments $\left(P_{1}=2.6\right.$ and $\left.P_{2}=1.0 \mathrm{mbar}\right)$. A fill/trap/ramp/wait sequence of 1-10/1-10/5-500/50 ms was used and an average resolving power of 180-250 was observed. A total of 500 accumulations and 10 frames were acquired per TIMS experiment. 
The measured mobility values $(K)$ were converted into collision cross sections (CCS, $\Omega$, $\AA^{2}$ ) using the Mason-Schamp equation:

$\Omega=\frac{(18 \pi)^{1 / 2}}{16} \frac{z}{\left(k_{B} T\right)^{1 / 2}}\left(\frac{1}{m_{I}}+\frac{1}{m_{b}}\right)^{1 / 2} \frac{1}{K_{0}} \frac{760}{P} \frac{T}{273.15} \frac{1}{N^{*}}$

where $z$ is the charge of the ion, $\mathrm{k}_{\mathrm{B}}$ is the Boltzmann constant, $N^{*}$ is the number density and $m_{I}$ and $m_{b}$ refer to the masses of the ion and bath gas, respectively. ${ }^{31}$

Collision induced activation experiments were performed prior to the TIMS-MS by varying the electric field between the capillary outlet ( $\left.V_{c a p}: 50-190 \mathrm{~V}\right)$, deflector plate $\left(V_{\text {def }}\right.$.60-200 V) and funnel entrance ( $\left.V_{\text {fun }}: 0-140 \mathrm{~V}\right)$ in $10 \mathrm{~V}$ steps.

Theoretical method

The 2ETL protein data bank entry for $\mathrm{UCH}$, was utilized as the starting structure for the molecular modelling. ${ }^{33}$ Briefly, the structures were cleaned and energy minimized using AMBER03 force field in YASARA software. The theoretical $\mathrm{CCS}_{\mathrm{N} 2}$ for each structure were calculated using the TM algorithm implemented in the iMOS software. ${ }^{34,35}$

\section{RESULTS AND DISCUSSION}

The mass spectrometry analysis of $\mathrm{UCH}$ in the positive mode under native-preserving solution conditions (i.e., $0 \% \mathrm{MeOH}, 10 \mathrm{mM} \mathrm{NH} 4 \mathrm{Ac}$ ), showed a single charge state distribution centered at the +9 charge state (Figure 6.1A). When the amount of organic content in the starting solution increases, a second distribution centered at the +21 charge state is observed. Interestingly, the charge states of the native-like species $(+8$ to +10$)$ are 
present at higher concentrations of organic content (up to $30 \% \mathrm{MeOH}$ ). This result suggest that the native structure of $\mathrm{UCH}$ is resistant to alcohol-induced denaturing, and this stability might be related to the presence of the $5_{2}$ knot embedded in the protein. When the starting solution contains up to $50 \% \mathrm{MeOH}$, a single charge state distribution is observed, between the +14 and +26 charge states. IMS experiments were performed on $\mathrm{UCH}$ as a function of the starting solution conditions (Figure 6.1B). The mobility profiles obtained for each charge state observed on each solvent condition were normalized to the respective intensity of each charge state in the mass spectrum. The overall $\mathrm{CCS}_{\mathrm{N} 2}$ profiles (black lines) were obtained by summation of the ion mobility resolved data (color lines). Similar to the mass spectrometry analysis on $\mathrm{UCH}$, the IMS experiments showed that the native-like conformation is resistant to solvent-induced denaturing, as the contribution of the profiles for the lower charge states is prevalent in the total IMS profile, even when the amount of $\mathrm{MeOH}$ is as high as $30 \%$. As expected, the relative intensity of the IMS profiles of the higher charge states (i.e., +11 to +26 ) increases with the addition of $\mathrm{MeOH}$ to the starting solution. As we have reported previously, the solvent-induced denaturing allows the exposure of basic sites for protonation during the nESI process. The coulombic repulsion driven by the additional positive charges causes an opening of the protein backbone, which further allows the study of the kinetically trapped intermediates of the unfolded states of the protein.

Further sampling of the conformational populations to study the folding pathway of $\mathrm{UCH}$ in the gas-phase was performed using CIA energy prior to the TIMS-MS analysis (Figures 6.2, S2, and S3). The profiles in figure 6.2 are as follows: the blue profiles were obtained 
when the starting solution did not contain any amount of methanol and the CIA energy was off (e.g., $V_{c a p}=50 \mathrm{~V}, V_{d e f}=60 \mathrm{~V}$, and $V_{f u n}=0 \mathrm{~V}$ ). The red profiles were obtained when $50 \%$ of the starting solution was methanol and the CIA was off. The yellow profiles were obtained when $10 \%$ of $\mathrm{MeOH}$ was added to the starting solution and the CIA energy was off. Finally, the green profiles were obtained when the starting solution had $10 \% \mathrm{MeOH}$ and the CIA energy was on. In fact, the additional CIA energy allowed the examination of additional kinetically trapped intermediates that might be part of the conformational space of both proteins. The amount of the organic content in each starting solution was selected to compare between native preserving conditions (e.g., 0\%) and denaturing conditions (e.g., 50\%, figure 6.2A), and the synergistic effect of a low amount of organic content (e.g., $10 \%$ ) when CIA is on (Figure 6.2B). The $\mathrm{CCS}_{\mathrm{N} 2}$ of the native +8 charge state of $\mathrm{UCH}$ is $\sim 2720 \AA^{2}$, and for the denatured +26 is $\sim 8730 \AA^{2}$. The theoretical $\mathrm{CCS}_{\mathrm{N} 2}$ measured using IMoS was $2642 \AA^{2}$. All the profiles obtained under the different experimental conditions align very closely to these values. The profiles for the lower $(+8$ to +10$)$ and higher $(+23$ to +26 ) charge states of $\mathrm{UCH}$ showed a single IMS band (Figure 6.2A and 6.2B), suggesting that at on these charge states the protein is populating a single conformation, either native-like or fully denatured. Additionally, the profiles obtained when the solution contained low amounts of $\mathrm{MeOH}$ (i.e., 0 and 10\%) displayed broad bands with identifiable peaks. In contrast, the profiles obtained when the amount of $\mathrm{MeOH}$ accounted for $50 \%$ of the starting solution are broad, and only display discrete bands after the charge state +17 . These results suggest that the knot embedded in the backbone of UCH provide greater conformational stability that is only disrupted by the solvation effect of the high amount of organic content. Interestingly, the native-like conformations where observed in the 
presence of $10 \% \mathrm{MeOH}$ when the CIA is off, past the +22 charge state until the +26 charge state (Figure 6.2B). However, when the CIA regime is on, these IMS bands disappear.

The thermal stability provided by the knot embedded in each protein was studied by performing IMS experiments on solutions with 0 and $10 \% \mathrm{MeOH}$ and incubation of the solutions at $20,35,50,65,80$, and $95^{\circ} \mathrm{C}$ (Figure 6.3). Examination of the profiles obtained for UCH under these conditions also showed that the native-like conformations are thermally stable, as the contribution of the +8 to +10 charge states is quite significant under all the experimental conditions analyzed (Figure 6.3A and 6.3B), while a progressive denaturing was also observed with the increasing temperature and in the presence of organic content in the starting solution. That is, a synergistic effect between temperature and organic content on the protein unfolding was evident when the starting solution also contains a small amount of $\mathrm{MeOH}$. When the solution does not contain $\mathrm{MeOH}$, the transition and unfolded conformations are evident as soon as the temperature is increased to $35^{\circ} \mathrm{C}$. In a similar way, profiles for the same conformations are observed whe the solution contains $10 \% \mathrm{MeOH}$ but the temperature is only $20^{\circ} \mathrm{C}$. When the solution without $\mathrm{MeOH}$ is heated, the intensity of the higher charge states increases, but is always lower than the intensity of the native-like charge states. In contrast, the synergistic effect of the $\mathrm{MeOH}$ on the thermal stability of the protein causes a rapid increase of the intensity of the denatured conformations Interestingly, the IMS bands for the native-like conformations are always present, even when the temperature is as high as $95^{\circ} \mathrm{C}$. This result suggests that the $5_{2}$ knot in UCH does indeed confer additional stability that protects the integrity of the protein under thermally-denaturing conditions. 
This discussion can be followed by the study of the stability and integrity of the knot core by enzymatic digestion of the protein termini using carboxypeptidase Y (CPDY). This region is defined using algorithms described by the mathematical field of knot theory, which studies the entanglement in closed chains, but has been modified to identify the residues that make-up the core in open polypeptide chains. ${ }^{36,37}$ By studying the fragments of the enzymatic digestion reaction of UCH with CPDY using IMS-MS, we can accurately determine which fragments are retaining the native conformation and integrity of the knot core. The high resolution of TIMS-MS allows the analysis of all the digestion products in terms of their size (CCS). Then it is possible to identify which fragments unfold, collapse, or retain the integrity of the core by comparing between the experimental and theoretical $\mathrm{CCS}_{\mathrm{N} 2}$ of the models generated by MD for each one of the observed fragments. The $5_{1}$ knot core found in the recombinant UCH is 220 amino acids long, between residues 22 and 241 (Figure 6.4B, cyan and magenta region). A slipknot is found between residues 169 and 249. This means that, when a residue is removed from this section, the $5_{1}$ knot will untangle and fold onto a $3_{1}$ knot. TIMS-MS analysis of the digestion showed that after $18 \mathrm{~h}$ of incubation, the reaction yielded 43 fragments with a maximum of 99 residues removed from the C-terminal, 28 from the $\mathrm{N}$-terminal, and 179 from both termini concomitantly (Figures 6.4B and S4A). Our results showed that the fragments containing a knot in their structure had a larger size than globular proteins of similar mass (Figure 6.4A, blue trendline). Moreover, the fragments that lost the integrity provided by the knot core, collapsed while a rearrangement of their overall structure adopts a conformation smaller than expected if the protein was globular (Figure 6.4A, black circles). Among the 43 fragments, 8 conserved the knot integrity in their structure, while the knot was shown to be 
conserved for multiple charge states of three of the fragments (Figures 6.4A, black stars and $4 \mathrm{C}$ ). Only the native charge states of the full sequence (i.e., +8 to +10 ) displayed a knotted conformation, while the rest of the fragments unfolded and/or collapsed, evidenced by multiple conformations (Figure 6.4C). All the fragments containing the knot core in their structure display a trend (Figure 6.4A, grey line), while is considered that the structures with CCS above this threshold unfold due the loss of the knot core.

\section{CONCLUSIONS}

This work provides insight into the unique structural integrity of the knot protein UCH in the gas-phase. The high ion mobility resolving power of the TIMS analyzer allowed the identification of detailed mobility bands between the +8 to +26 charge states as a function of the starting solution conditions and the activation energy. The experimental results showed that the 51 knot observed in the native-like conformations confers additional stability to the overall integrity of $\mathrm{UCH}$, which prevents the structural denaturing of the protein when the starting solutions contain high amounts of organic content or are examined under high energy regime conditions. This additional stability is also manifest when the starting solutions are subjected to temperatures that deviate from what is expected physiologically. It is noteworthy that the native-like conformations are observed even when the temperature of a solution with $\mathrm{MeOH}$ is increased, while the synergistic effect of these two conditions should cause a rapid denaturing of the tertiary structure. The calculated $\mathrm{CCS}_{\mathrm{N} 2}$ of molecular models for the native and denatured forms of $\mathrm{UCH}$ (i.e., +8 and +26 , respectively) is within $5 \%$ of the experimentally measured $\mathrm{CCS}_{\mathrm{N} 2}$. 
Finally, the stability and integrity of the knot core was also studied. Removal of the knot tails by enzymatic digestion with CPDY allowed the analysis of charged fragments that contained the residues that makeup the knot core. The experimentally measured $\mathrm{CCS}_{\mathrm{N} 2}$ of each fragment was compared with theoretically calculated $\mathrm{CCS}_{\mathrm{N} 2}$ of models that matched the amino acid length of each fragment. A total of 43 fragments were observed, while the knot was found to be intact in 8 of them. Our results suggest that the loss of the knot core can either cause a structural collapse or an unfolding of protein. The collapsed structures are found to be smaller than globular proteins of the similar mass.

The analytical workflow implemented in this work describes powerful TIMS-based strategies for the comprehensive analysis of the complex conformations found in many biomolecules. Empirical information was obtained by exploring the conformational landscape generated by the conditions of the starting solutions, the activation energy regime, the thermal stability of the protein conformation, and the isolation of the structural regions of interest by enzymatic digestion. Combined with molecular dynamics and computational methods that calculate the $\mathrm{CCS}_{\mathrm{N} 2}$ of molecular models, is possible to obtain information on the structural makeup of these interesting topologies.

\section{ASSOCIATED CONTENT}

Scheme of the TIMS cell, contour plots of each charge state as a function of the staring solution conditions and CIA energy, and summary of the information on each fragment obtained after digestion with CPDY. 


\section{FUNDING SOURCES}

The authors declare no competing financial interest. The authors acknowledge the financial support from the National Institute of Health (R00GM106414), a Bruker Daltonics Inc. fellowship, and the National Science Foundation Division of Chemistry, under CAREER award CHE-1654274, with co-funding from the Division of Molecular and Cellular Biosciences to FFL.

\section{ACKNOWLEDGMENTS}

The authors would like to acknowledge the Instructional \& Research Computing Center (IRCC) at Florida International University for providing high performance computing resources that have contributed to the research results reported within this research. 
Figure 6.1 Typical mass spectra of $\mathrm{UCH}$ in the positive mode as a function of the starting solvent conditions. Overall $\mathrm{CCS}_{\mathrm{N} 2}$ profiles of $\mathrm{UCH}$ (black lines) obtained by summation of the intensity-normalized IMS resolved data (color lines).
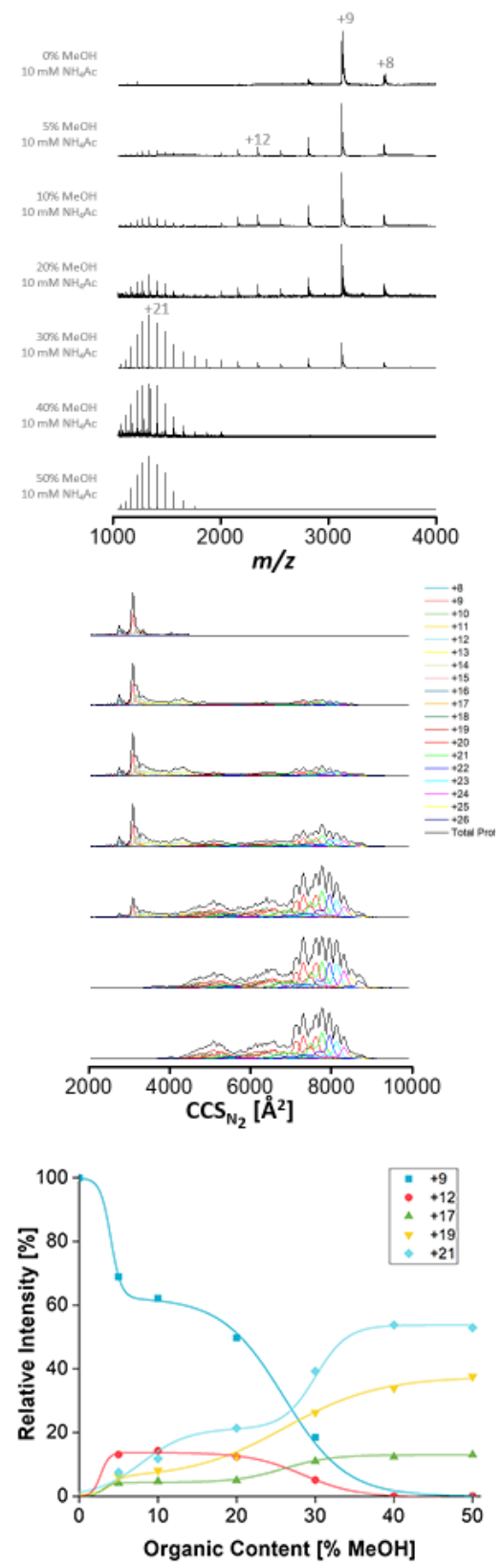
Figure 6.2 IMS profiles of the +8 to +26 charge states of $\mathrm{UCH}$ as a function of the organic content (e.g., \% MeOH) in the starting solution. IMS profiles of the +8 to +26 charge states of UCH as a function of the CIA regime (e.g., on/off) for a solution with $10 \% \mathrm{MeOH}$. The blue profiles were obtained when the starting solution did not contain any amount of methanol and the CIA energy was off (e.g., $V_{c a p}=50 \mathrm{~V}, V_{d e f}=60 \mathrm{~V}$, and $\left.V_{f u n}=0 \mathrm{~V}\right)$. The red profiles were obtained when $50 \%$ of the starting solution was methanol and the CIA was off. The yellow profiles were obtained when $10 \%$ of $\mathrm{MeOH}$ was added to the starting solution and the CIA energy was off. Finally, the green profiles were obtained when the starting solution had $10 \% \mathrm{MeOH}$ and the CIA energy was on.

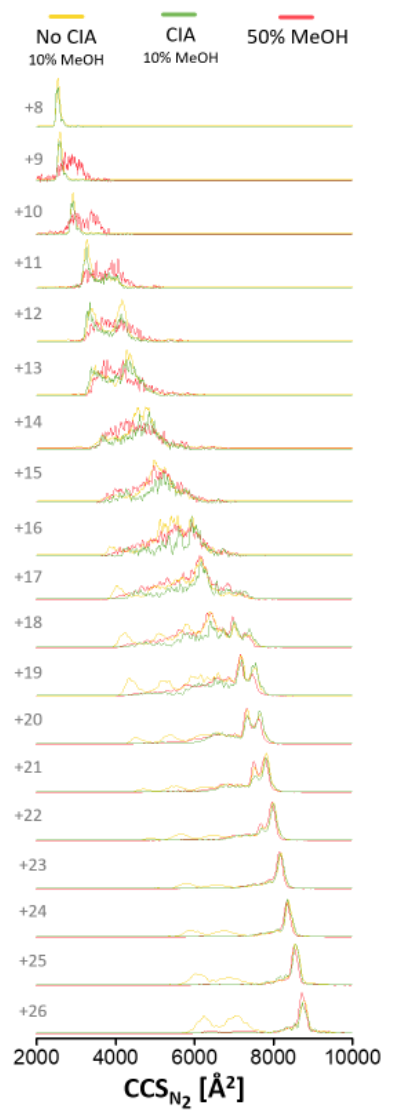


Figure 6.3 Overall $\mathrm{CCS}_{\mathrm{N} 2}$ profiles for $\mathrm{UCH}$ (black lines) obtained by summation of the intensity-normalized IMS resolved data (color lines) obtained as a function of the temperature of the starting solution with $0 \% \mathrm{MeOH}$, and $10 \% \mathrm{MeOH}$.
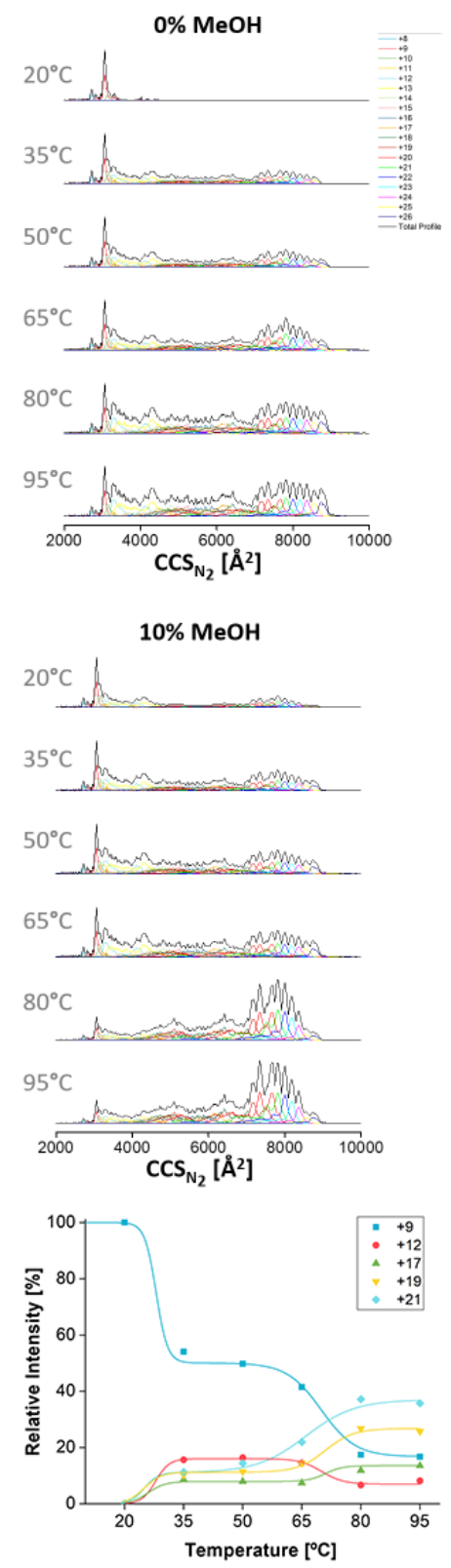
Figure 6.4 Digestion of $\mathrm{UCH}$ with $\mathrm{CPDY}$. A: $\mathrm{CCS}_{\mathrm{N} 2}$ distribution of the observed fragments as a function of the mass of each fragment. Orange circles: undigested protein; Green triangles: fragments with residues removed exclusively from the C-terminal; Red triangles: fragments with residues removed exclusively from the N-terminal; Blue rhomboids: fragments with residues removed from the both termini; Black circles: fragments where a structural collapse is observed; Black stars and grey line: fragments where the knot is preserved after the digestion; Blue line: distribution of reported $\mathrm{CCS}_{\mathrm{N} 2}$ for globular proteins. B: Sequence coverage by the digestion. C: IMS profiles of the fragments that preserve the knot.
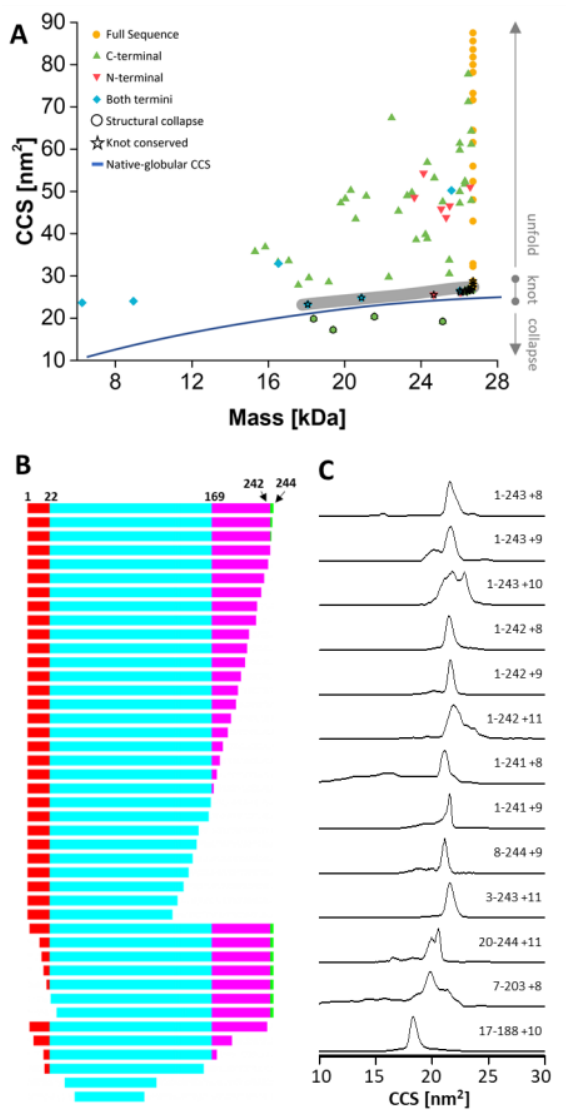


\section{LIST OF REFERENCES}

(1) Russel, D.; Lasker, K.; Phillips, J.; Schneidman-Duhovny, D.; Velazquez-Muriel, J. A.; Sali, A. Curr Opin Cell Biol 2009, 21, 97-108.

(2) Dill, K. A.; MacCallum, J. L. Science 2012, 338, 1042-1046.

(3) Schenk, E. R.; Mendez, V.; Landrum, J. T.; Ridgeway, M. E.; Park, M. A.; FernandezLima, F. Anal Chem 2014, 86, 2019-2024.

(4) Schenk, E. R.; Ridgeway, M. E.; Park, M. A.; Leng, F.; Fernandez-Lima, F. Anal Chem 2014, 86, 1210-1214.

(5) Yeates, T. O.; Norcross, T. S.; King, N. P. Current opinion in chemical biology 2007, $11,595-603$.

(6) Bolinger, D.; Sulkowska, J. I.; Hsu, H. P.; Mirny, L. A.; Kardar, M.; Onuchic, J. N.; Virnau, P. PLoS Comput Biol 2014, 6, e1000731.

(7) Millett, K. C.; Rawdon, E. J.; Stasiak, A.; Sulkowska, J. I. Biochemical Society transactions 2013, 41, 533-537.

(8) Virnau, P.; Mirny, L. A.; Kardar, M. PLoS Comput Biol 2006, 2, e122.

(9) Taylor, W. R. Computational biology and chemistry 2007, 31, 151-162.

(10) Sulkowska, J. I.; Sulkowski, P.; Onuchic, J. Proc Natl Acad Sci U S A 2009, 106, 3119-3124.

(11) Mallam, A. L.; Jackson, S. E. J Mol Biol 2005, 346, 1409-1421.

(12) Mallam, A. L. FEBS J 2009, 276, 365-375.

(13) Virnau, P.; Mallam, A.; Jackson, S. Journal of physics. Condensed matter : an Institute of Physics journal 2011, 23, 033101.

(14) Andersson, F. I.; Pina, D. G.; Mallam, A. L.; Blaser, G.; Jackson, S. E. FEBS J 2009, $276,2625-2635$.

(15) Misaghi, S.; Galardy, P. J.; Meester, W. J.; Ovaa, H.; Ploegh, H. L.; Gaudet, R. J Biol Chem 2005, 280, 1512-1520.

(16) Love, K. R.; Catic, A.; Schlieker, C.; Ploegh, H. L. Nature chemical biology 2007, 3, 697-705. 
(17) Fernandez-Lima, F.; Blase, R. C.; Russell, D. H. Int. J. Mass Spectrom. 2011, 298, 111-118.

(18) Fernandez-Lima, F.; Kaplan, D. A.; Suetering, J.; Park, M. A. Int. J. Ion Mobil. Spectrom. 2011, 14, 93-98.

(19) Hernandez, D. R.; Debord, J. D.; Ridgeway, M. E.; Kaplan, D. A.; Park, M. A.; Fernandez-Lima, F. Analyst 2014, 139, 1913-1921.

(20) Fernandez-Lima, F. International Journal for Ion Mobility Spectrometry 2016, 19, 6567.

(21) Benigni, P.; Fernandez-Lima, F. Analytical Chemistry 2016, 88, 7404-7412.

(22) Molano-Arevalo, J. C.; Jeanne Dit Fouque, K.; Pham, K.; Miksovska, J.; Ridgeway, M. E.; Park, M. A.; Fernandez-Lima, F. Anal Chem 2017, 89, 8757-8765.

(23) Schenk, E. R.; Mendez, V.; Landrum, J. T.; Ridgeway, M. E.; Park, M. A.; FernandezLima, F. Anal. Chem. 2014, 86, 2019-2024.

(24) Schenk, E. R.; Ridgeway, M. E.; Park, M. A.; Leng, F.; Fernandez-Lima, F. Anal. Chem. 2014, 86, 1210-1214.

(25) Garabedian, A.; Butcher, D.; Lippens, J. L.; Miksovska, J.; Chapagain, P.; Fabris, D.; Ridgeway, M. E.; Park, M. A.; Fernandez-Lima, F. Physical Chemistry Chemical Physics 2016, 18, 26691-26702.

(26) Pu, Y.; Ridgeway, M. E.; Glaskin, R. S.; Park, M. A.; Costello, C. E.; Lin, C. Analytical Chemistry 2016, 88, 3440-3443.

(27) Fernandez-Lima, F.; Kaplan, D. A.; Suetering, J.; Park, M. A. International journal for ion mobility spectrometry : official publication of the International Society for Ion Mobility Spectrometry 2011, 14, 93-98.

(28) Fernandez-Lima, F. A.; Kaplan, D. A.; Park, M. A. The Review of scientific instruments 2011, 82, 126106.

(29) Fernandez-Lima, F. A.; Kaplan, D. A.; Park, M. A. Rev. Sci. Instrum. 2011, 82, 126106.

(30) Molano-Arevalo, J. C.; Hernandez, D. R.; Gonzalez, W. G.; Miksovska, J.; Ridgeway, M. E.; Park, M. A.; Fernandez-Lima, F. Anal. Chem. 2014, 86, 10223-10230.

(31) McDaniel, E. W.; Mason, E. A. Mobility and diffusion of ions in gases; John Wiley and Sons, Inc., New York: New York, 1973, p 381.

(32) United States, 1999. 
(33) Lim, K.; Zhang, H.; Tempczyk, A.; Krajewski, W.; Bonander, N.; Toedt, J.; Howard, A.; Eisenstein, E.; Herzberg, O. Proteins 2003, 51, 56-67.

(34) Larriba, C.; Hogan, C. J., Jr. J. Phys. Chem. A 2013, 117, 3887-3901.

(35) Larriba, C.; Hogan, C. J. J. Comput. Phys. 2013, 251, 344-363.

(36) Taylor, W. R. Nature 2000, 406, 916-919.

(37) Sulkowska, J. I.; Rawdon, E. J.; Millett, K. C.; Onuchic, J. N.; Stasiak, A. Proc Natl Acad Sci U S A 2012, 109, E1715-1723. 
CHAPTER 7.

ELUCIDATION OF THE STRUCTURAL INTEGRITY AND STABILITY OF THE KNOT PROTEIN HALO ACID DEHALOGENASE USING TRAPPED ION MOBILITY SPECTROMETRY - MASS SPECTROMETRY 


\begin{abstract}
Protein knots were regarded as structural impossibilities, until they were observed embedded in naturally occurring proteins. The most complex knot found in proteins so far is the Stevedore knot, a $6_{1}$ knot found in the core of the $\alpha$-haloacid dehalogenase (DehI). In the present work, the kinetically trapped intermediates of the knot protein DehI were investigated using trapped ion mobility - mass spectrometry (TIMS-MS) as a function of the starting solvent conditions (i.e., organic content), collision induced activation (CIA), and starting solution temperature. Our results suggest that the stability provided by the knot core is not as high as expected for an entangled structure. A rapid unfolding and expansion of the native-like conformations observed was evident under a wide variety of experimental conditions. The stability and integrity of the knot core was also assessed by enzymatic digestion using carboxypeptidase Y (CPDY). Molecular dynamics simulations were used to propose candidate models to assess the knot integrity of the observed fragments. A total of 50 fragments were observed after the enzymatic reaction, while the knot was found to be stable in 12 of the fragments.
\end{abstract}




\section{INTRODUCTION}

Several protein structures incorporate a rather unusual structural feature -a knot in the polypeptide backbone. ${ }^{1}$ For the past 20 years, this fascinating group of proteins with knotted topologies have been identified to occur naturally, but for a long time, it was thought that these complex structures where to impossible to exist ${ }^{2}$ The knot proteins went undetected for many years, as the problem of identifying the knots by visual inspection is tremendously difficult. ${ }^{3}$ These intricate topological barriers can lead to energy barriers that difficult the structural characterization of the protein, and despite the research on these structures, questions about how and when in the folding process the knots are formed still remain unanswered by the experiments carried out so far. ${ }^{4}$

One of the most complex knots found in proteins so far is the Stevedore knot, a $6_{1}$ knot found in the core of the $\alpha$-haloacid dehalogenase (DehI). ${ }^{5}$ The structure of DehI contains a knot with six crossings. To study the folding of this complex knot, simulations with a structure-based coarse-grained model were performed, showing a possible mechanism by which the knot forms when a large twisted loop in the protein flips over another previously twisted loop, creating the knot in a single movement. ${ }^{5}$ The folding pathway of DehI is

different from other knot proteins that utilize partially knotted intermediates. ${ }^{5}$ Until now, no experimental results have been reported on the structural analysis of this protein.

A new approach to study the folding pathway of proteins, especially those with intricate structures is trapped ion mobility spectrometry (TIMS-MS). In TIMS-MS, a constant electric field component holds ions stationary against a moving buffer gas while a quadrupolar rf field radially confines them to avoid losses of the ions to the 
electrodes.36,37 The TIMS-MS systems have proven useful for the rapid separation and high-resolution structural elucidation of biomolecules, ${ }^{6-13}$ for example: screening ${ }^{6}$ and targeted $^{14}$ analysis of complex mixtures, tracking the isomerization kinetics, ${ }^{8-10}$ and characterizing the conformational spaces of peptides, ${ }^{15}$ DNA, ${ }^{11}$ proteins, ${ }^{16}$ and macromolecular complexes in native and denatured states. ${ }^{17}$

This study combines the structure elucidation capabilities of TIMS-MS to explain the conformational dynamics of DehI, while providing unique insights into the folding pathway and stabilization mechanisms of the knot core. Our results showed that this approach provides an invaluable insight necessary for the analysis of these intricate knotted topologies. In the following discussion, a special emphasis will be placed on the structural integrity and stability of the knot core embedded in DehI.

\section{MATERIALS AND METHODS}

Materials and Reagents

All solvents used were analytical grade or better and purchased from Fisher Scientific (Pittsburg, PA). DehI was expressed recombinantly in Escherichia coli BL21(DE3) and purified using a nickel column. The protein was shown to be pure using both SDS/PAGE and MS analysis. A stock solution of ??? was prepared in a buffer of $10 \mathrm{mM}$ ammonium acetate $\left(\mathrm{NH}_{4} \mathrm{Ac}\right)$, dialyzed against the same buffer and diluted to a final concentration of $10 \mu \mathrm{M}$ in10 mM of $\mathrm{NH}_{4} \mathrm{Ac}, 95: 5,90: 10,80: 20,70: 30,60: 40$, and 50:50 (v/v) $\mathrm{H}_{2} \mathrm{O} / \mathrm{MeOH}$. Nano-ESI emitters were pulled from quartz capillaries (O.D.: $1.0 \mathrm{~mm}$ and I.D.: $0.70 \mathrm{~mm}$ ) using Sutter Instruments Co. P2000 laser puller. Low-concentration Tuning Mix 
calibration standard (TuneMix, G24221A) was purchased from Agilent Technologies (Santa Clara, CA). A stock solution of carboxypeptidase y (CPDY) was prepared with a final concentration of $1 \mathrm{mg} / \mathrm{mL}$. Prior incubation, $2 \mu \mathrm{L}$ of CPDY were added to a solution of $200 \mu \mathrm{L}$ of DehI. Incubation was performed at $26.5^{\circ} \mathrm{C}$ for $18 \mathrm{~h}$. After the incubation, the enzymatic reaction was quenched by the addition of $20 \mu \mathrm{L}$ of glacial acetic acid.

\section{CIA-TIMS-MS Experiments}

We employed a custom nESI-TIMS unit coupled to an Impact Q-TOF mass spectrometer (Bruker, Billerica, MA). ${ }^{18,19}$ The TIMS unit is run by custom software in LabView (National Instruments) synchronized with the MS platform controls. ${ }^{19}$ Sample aliquots (10 $\mu \mathrm{L})$ were loaded in a pulled-tip capillary biased at $\sim 1000 \mathrm{~V}$ to the MS inlet. Details regarding the TIMS operation compared to traditional IMS can be found elsewhere. ${ }^{20-24}$ Briefly, TIMS mobility separation is based on holding the ions stationary using an electric field against a moving buffer gas. ${ }^{25}$ The mobility, $K$, of an ion in a TIMS cell is described by:

$K=\frac{v_{g}}{E}=\frac{A}{\left(V_{\text {elution }}-V_{\text {out }}\right)}$

where $v_{g}, E, V_{\text {elution }}$ and $V_{\text {out }}$ are the gas velocity, applied electric field, elution voltage and base voltage, respectively. The constant $A$ was determined using a Tuning Mix calibration standards $\left(m / z, 322 K_{o}=1.376 \mathrm{~cm}^{2} \mathrm{~V}^{-1} \mathrm{~s}^{-1}, m / z 622 K_{o}=1.013 \mathrm{~cm}^{2} \mathrm{~V}^{-1} \mathrm{~s}^{-1}\right.$, and $m / z 922 \mathrm{~K}_{o}=$ $\left.0.835 \mathrm{~cm}^{2} \mathrm{~V}^{-1} \mathrm{~s}^{-1}\right) \cdot{ }^{22,26}$ 
The same rf ( $880 \mathrm{kHz}$ and $280 \mathrm{Vpp})$ was applied to all electrodes including the entrance funnel, the ion mobility separating section, and the exit funnel (Figure S7.1). TIMS separation was performed using nitrogen as a bath gas at $300 \mathrm{~K}$, and the gas flow velocity was held constant in all the experiments $\left(P_{1}=2.6\right.$ and $\left.P_{2}=1.0 \mathrm{mbar}\right)$. A fill/trap/ramp/wait sequence of 1-10/1-10/5-500/50 ms was used and an average resolving power of 180-250 was observed. A total of 500 accumulations and 10 frames were acquired per TIMS experiment.

The measured mobility values $(K)$ were converted into collision cross sections (CCS, $\Omega$, $\AA^{2}$ ) using the Mason-Schamp equation:

$\Omega=\frac{(18 \pi)^{1 / 2}}{16} \frac{z}{\left(k_{B} T\right)^{1 / 2}}\left(\frac{1}{m_{I}}+\frac{1}{m_{b}}\right)^{1 / 2} \frac{1}{K_{0}} \frac{760}{P} \frac{T}{273.15} \frac{1}{N^{*}}$

where $z$ is the charge of the ion, $\mathrm{k}_{\mathrm{B}}$ is the Boltzmann constant, $N^{*}$ is the number density and $\mathrm{m}_{\mathrm{I}}$ and $\mathrm{m}_{\mathrm{b}}$ refer to the masses of the ion and bath gas, respectively. ${ }^{25}$

Collision induced activation experiments were performed prior to the TIMS-MS by varying the electric field between the capillary outlet $\left(V_{c a p}: 50-190 \mathrm{~V}\right)$, deflector plate $\left(V_{\text {def }}\right.$ 60-200 V) and funnel entrance $\left(V_{\text {fun }}: 0-140 \mathrm{~V}\right)$ in $10 \mathrm{~V}$ steps.

Theoretical method

The 3BJX protein data bank entry for DehI was utilized as the starting structures. ${ }^{27}$ Briefly, the structures were cleaned and energy minimized using AMBER03 force field in YASARA software. The theoretical $\mathrm{CCS}_{\mathrm{N} 2}$ for each structure were calculated using the TM algorithm implemented in the iMOS software. ${ }^{28,29}$ 


\section{RESULTS AND DISCUSSION}

The mass spectrum of DehI (Figure 7.1A) in positive mode showed a single charge state distribution for the native-like charge states $(+8$ to +12$)$ when the organic content in the starting solution is low (i.e., 0 and $5 \% \mathrm{MeOH}$ ). When the amount of $\mathrm{MeOH}$ increases to $10 \%$, the +8 and +9 charge states vanish, and the distribution shifts to encompass the charge states between +10 to +20 . It has been reported previously that the $6_{1}$ knot of DehI is formed after a single flipping event. ${ }^{5}$ Our results suggest that this event is easily undone when the solvation effect of a small amount of $\mathrm{MeOH}$ in the starting solution, explaining the sudden disappearance of the native-like species. At $20 \% \mathrm{MeOH}$, the distribution shifts from the +13 to give rise to two distributions, centered at +16 and +20 respectively. Further increases of $\mathrm{MeOH}$ are reflected in a diminishing intensity of the lower charge states $(+10$ to +12 ), and a single envelope is observed when the organic content constitutes $50 \%$ of the starting solution, encompassing the charge states between +14 to +31 .

IMS experiments were performed for DehI as a function of the starting solution conditions (Figure 7.1B). The mobility profiles obtained for each charge state observed on each solvent condition were normalized to the respective intensity of each charge state in the mass spectrum. The overall $\mathrm{CCS}_{\mathrm{N} 2}$ profiles (black lines) were obtained by summation of the ion mobility resolved data (color lines). The IMS profiles of the native-like charge states of DehI disappear quickly with the additional organic content in the solution. As mentioned previously, it has been reported that the knot on DehI might be formed after a single flipping event of the backbone. Our results suggest that the solvation effect of a low amount of methanol (i.e., up to 5\%) is enough to disrupt the intramolecular interactions 
that help conserve the integrity of the spatial constraint provided by the Stevedore knot. Further additions of methanol to the starting solution favor the denatured states of the protein, evidenced by the increased intensity of the IMS profiles of the higher charge states. As expected, the relative intensity of the IMS profiles of the higher charge states (i.e., +13 to +31 ) increases with the addition of $\mathrm{MeOH}$ to the starting solution. As it has been reported previously, the solvent-induced denaturing allows the exposure of basic sites for protonation during the nESI process. The coulombic repulsion driven by the additional positive charges causes an unfolding of the protein backbone, which further allows the study of the kinetically trapped intermediates of the denatured states of the protein.

Further sampling of the conformational populations to study the folding pathway of the knot protein DehI in the gas-phase was performed using CIA energy prior to the TIMSMS analysis (Figures 7.2, S7.2, S7.3). The profiles in figure 7.2 are as follows: the blue profiles were obtained when the starting solution did not contain any amount of methanol and the CIA energy was off (e.g., $V_{c a p}=50 \mathrm{~V}, V_{\text {def }}=60 \mathrm{~V}$, and $V_{f u n}=0 \mathrm{~V}$ ). The red profiles were obtained when $50 \%$ of the starting solution was methanol and the CIA was off. The yellow profiles were obtained when $10 \%$ of $\mathrm{MeOH}$ was added to the starting solution and the CIA energy was off. Finally, the green profiles were obtained when the starting solution had $10 \% \mathrm{MeOH}$ and the CIA energy was on. In fact, the additional CIA energy allowed the examination of additional kinetically trapped intermediates that might be part of the conformational space of both proteins. The amount of the organic content in each starting solution was selected to compare between native preserving conditions (e.g., 0\%) and denaturing conditions (e.g., 50\%), and the synergistic effect of a low amount of organic 
content (e.g., 10\%) when CIA is on. IMS experiments on DehI showed that the $\mathrm{CCS}_{\mathrm{N} 2}$ of the native +8 charge state is $\sim 3210 \AA^{2}$, and for the fully denatured +31 is $\sim 15330 \AA^{2}$. The theoretical $\mathrm{CCS}_{\mathrm{N} 2}$ measured using IMoS was $3278 \AA^{2}$ (Figure 7.2A). Our results showed that the structural variability of DehI is not as diverse, as mostly broad bands are observed while a rapid unfolding occurs as a function of the increasing charge state. The profiles obtained under the different experimental conditions when the amount of $\mathrm{MeOH}$ is low, are quite broad and only narrow bands are observed for some of the lower $(+8$ to +15$)$ and higher $\left(+26\right.$ to $\left.{ }^{-}+31\right)$ charge states. In contrast, the profiles obtained when the solution contains $50 \% \mathrm{MeOH}$ where only for the charge states above +16 , and they showed a narrowing of the bands after the +19 charge state, only populating the trend that leads to the fully denatured conformations. This result is in agreement with our previous assertion that DehI is more susceptible to solvent-induced denaturing, as the knot does not provide enough stability to the structural integrity of the protein. No major changes were observed as a function of the CIA regime (Figure 7.2B). That is, the mobility profiles obtained under the CIA regime are broad and very similar to the profiles obtained without CIA regime for the same solution conditions. The main differences between CIA on and off can be observed for the profiles of the +15 to +17 charge states, where the bands obtained under CIA on are broader, concealing a small feature observed with CIA off for the same charge states.

The thermal stability provided by the knot was studied by performing IMS experiments on solutions with 0 and $10 \% \mathrm{MeOH}$ and incubation of the solutions at 20,35, 50, 65, 80, and $95^{\circ} \mathrm{C}$ (Figure 7.3). The native-like conformations of DehI were only observed when the 
solution did not contained $\mathrm{MeOH}$ and the temperature was $20^{\circ} \mathrm{C}$. A progressive denaturing as a function of the temperature was observed for both proteins. However, this synergistic effect between temperature and organic content was very pronounced for DehI, as only the transition and denatured conformations were observed after the solution was heated to $65^{\circ} \mathrm{C}$ when the solution had $10 \% \mathrm{MeOH}$. These results suggests that the DehI is heat sensitive, as the structure of the protein is easily disrupted when the temperature increases slightly, evidenced by the disappearance of the IMS bands that correspond to the native-like conformations. This observation can be explained by analyzing the amino acid composition of DehI. Essentially, the chain is composed of two similar regions that form a pseudo-dimer while they are connected by a proline rich arc. Additionally, it has been theorized that the final knotting event that takes place in the folding pathway of DehI, is a single flip event. Our results suggest that is somewhat easy to undo this flip event, causing a rapid unfolding of the molecular structure.

It has been discussed that every knot protein contains a region within called the knot core, which is defined as the shortest sub-chain within the protein for which a knot is stable. To evaluate the occurrence of a knot in a protein, several algorithms have been developed to analyze the multitude of protein structures stored in databases, such as the PDB database. However, the reliability of these algorithms is far from high, as the implementation of the mathematical models on knots require a compromise when evaluated open chains, as found in naturally occurring proteins, instead of closed chains, as the models describe. In this study, we implemented a combination of enzymatic digestion using carboxypeptidase $\mathrm{Y}$ (CPDY) and TIMS-MS to measure the size, in terms of $\mathrm{CCS}_{\mathrm{N} 2}$, of the charged fragments 
resulting from the biochemical reaction. Then, by comparing the empirical size with the theoretical CCS of molecular models generated using MD, we can determine when the core is stable and confers additional structural integrity to the protein (Figure 7.4). The Stevedore $6{ }_{1}$ knot core found in the recombinant DehI is 214 amino acids long, between residues 93 and 306 (Figure 7.4B, blue region). An enzymatic digestion of DehI using CPDY was performed to isolate the core from the knot tails (Figures 7.4, S7.4, and Table S7.1). TIMS-MS analysis of the digestion showed that after $18 \mathrm{~h}$ of incubation, the reaction yielded 50 fragments with a maximum of 178 residues missing from the $\mathrm{C}$-terminal, 38 from the N-terminal, and 126 from both termini concomitantly. Our results showed that, in contrast to observations made on the enzymatic digestion of YibK and $\mathrm{UCH}$, the fragments that lost the integrity provided by the knot core, did not collapsed, but opened even further towards large-size unfolded conformations. Of the observed fragments, 12 conserved the structural integrity of the knot in their structure (Figures 7.4A, black stars and 4B). All the fragments containing the knot core in their structure display a trend (Figure 7.4A, grey line), while is considered that the structures with CCS above this threshold unfold due the loss of the knot core.

\section{CONCLUSIONS}

In the present work we examined the structural integrity provided by the Stevedore 61 knot to the conformation of the protein halo acid dehalogenase, DehI. A plethora of TIMS-MS techniques were used to assess the structural integrity of the protein. The high resolution of the TIMS analyzer allowed the examination of the trapped kinetic intermediates present in the 23 charge states (e.g., +8 to +31 ) generated by nESI as a function of the solution 
conditions (i.e., $0-50 \% \mathrm{MeOH}$ ), and the collision induced activation energy (CIA, $\mathrm{V}_{\text {def }} 60$ to $200 \mathrm{~V}$ ). The results of these experiments showed that the native-like conformations (charge states +9 to +12 ) are not stable under a wide variety of environments, but to only native-preserving solution and energy conditions. This observation were also evident when evaluating the thermal stability of the protein. Our observations showed that a slight change in the temperature of the starting solution (i.e., from 20 to $35^{\circ} \mathrm{C}$ ) was enough to cause a structural disruption of the native states, evidenced by the disappearance of the mobility bands for the aforementioned native-like charge states. Moreover, this effect was more pronounced by the synergistic action of a small addition of $\mathrm{MeOH}$ to the starting solution condition. This structural instability can be explained by analyzing the structural makeup if DehI, where a pseudodimer is formed when the two homologous ends of the protein, connect by a proline rich arc. This pseudodimer rapidly unfolds under the action of environmental factors in the solution, such as the solvation effect of the organic molecules or the increase of the vibrational energy, either by CIA or temperature of the solution.

The integrity of the knot core was also studied and the stability that this core grants to the overall molecular structure was assessed by removal of the knot tails by enzymatic digestion with CPDY. This allowed the analysis of charged fragments that contained the residues that makeup the knot core. The experimentally measured $\mathrm{CCS}_{\mathrm{N} 2}$ of each fragment was compared with theoretically calculated $\mathrm{CCS}_{\mathrm{N} 2}$ of models that matched the amino acid length of each fragment. A total of 50 fragments were observed, while the knot was found to be intact in 12 of them. Our results suggest that the loss of the knot core in DehI can only cause a structural unfolding of protein. 
This work provides, for the first time, a workflow that combines the analytical capabilities of the TIMS analyzer with the results from biochemical reactions that allowed the isolation of the knot core.

\section{ASSOCIATED CONTENT}

Scheme of the TIMS cell, contour plots of each charge state as a function of the staring solution conditions and CIA energy, and summary of the information on each fragment obtained after digestion with CPDY.

\section{FUNDING SOURCES}

The authors declare no competing financial interest. The authors acknowledge the financial support from the National Institute of Health (R00GM106414), a Bruker Daltonics Inc. fellowship, and the National Science Foundation Division of Chemistry, under CAREER award CHE-1654274, with co-funding from the Division of Molecular and Cellular Biosciences to FFL.

\section{ACKNOWLEDGMENTS}

The authors would like to acknowledge the Instructional \& Research Computing Center (IRCC) at Florida International University for providing high performance computing resources that have contributed to the research results reported within this research. 
Figure 7.1 Typical mass spectra of DehI in the positive mode as a function of the starting solvent conditions. Overall $\mathrm{CCS}_{\mathrm{N} 2}$ profiles of DehI (black lines) obtained by summation of the intensity-normalized IMS resolved data (color lines).
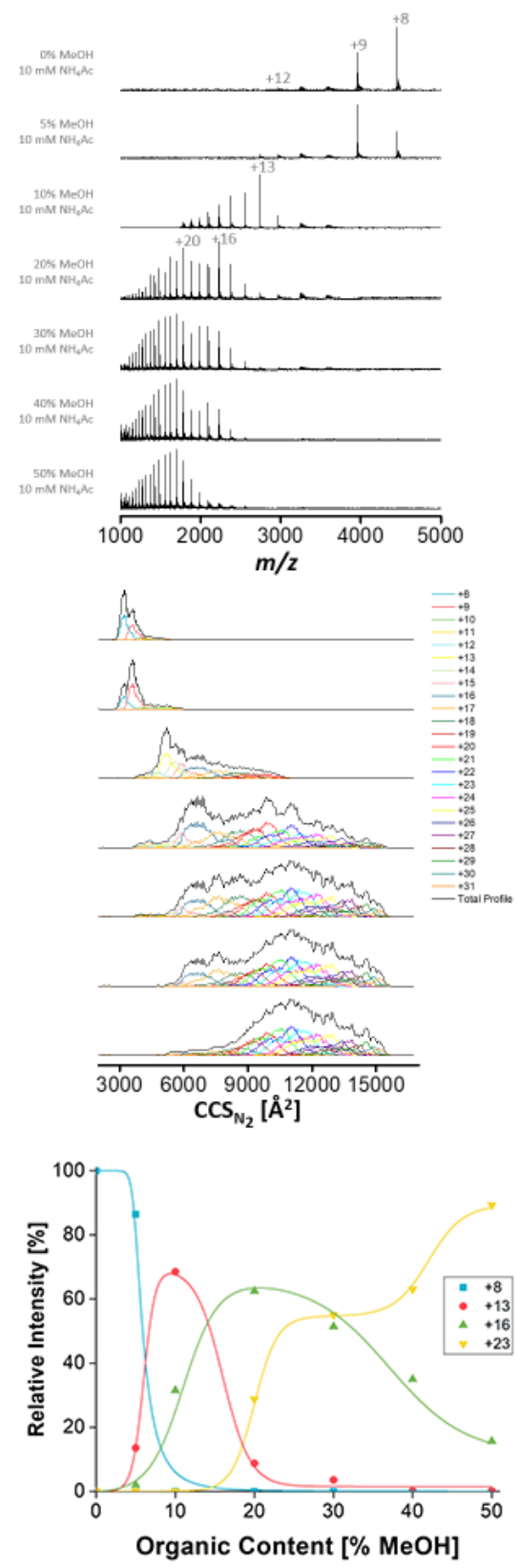
Figure 7.2 IMS profiles of the +8 to +31 charge states of DehI as a function of the organic content (e.g., \% MeOH) in the starting solution. IMS profiles of the +8 to +31 charge states of DehI as a function of the CIA regime (e.g., on/off) for a solution with $10 \% \mathrm{MeOH}$. The blue profiles were obtained when the starting solution did not contain any amount of methanol and the CIA energy was off (e.g., $V_{c a p}=50 \mathrm{~V}, V_{d e f}=60 \mathrm{~V}$, and $\left.V_{f u n}=0 \mathrm{~V}\right)$. The red profiles were obtained when $50 \%$ of the starting solution was methanol and the CIA was off. The yellow profiles were obtained when $10 \%$ of $\mathrm{MeOH}$ was added to the starting solution and the CIA energy was off. Finally, the green profiles were obtained when the starting solution had $10 \% \mathrm{MeOH}$ and the CIA energy was on.

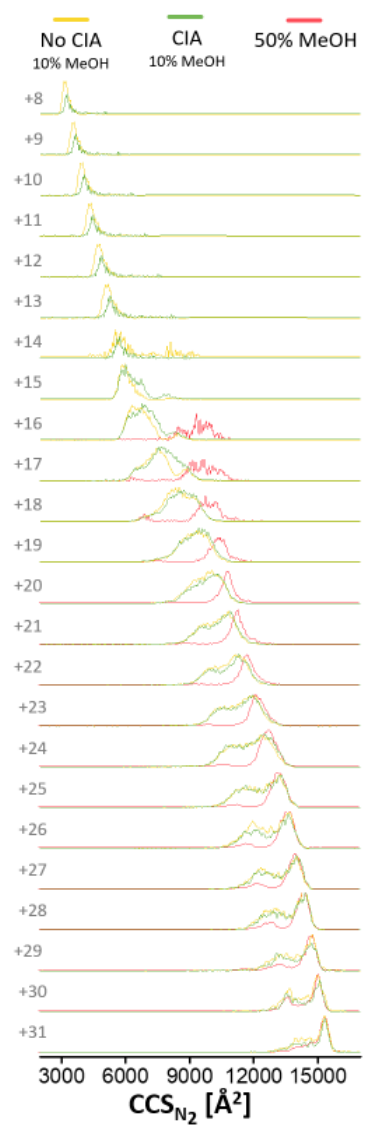


Figure 7.3 Overall $\mathrm{CCS}_{\mathrm{N} 2}$ profiles for DehI (black lines) obtained by summation of the intensity-normalized IMS resolved data (color lines) obtained as a function of the temperature of the starting solution.
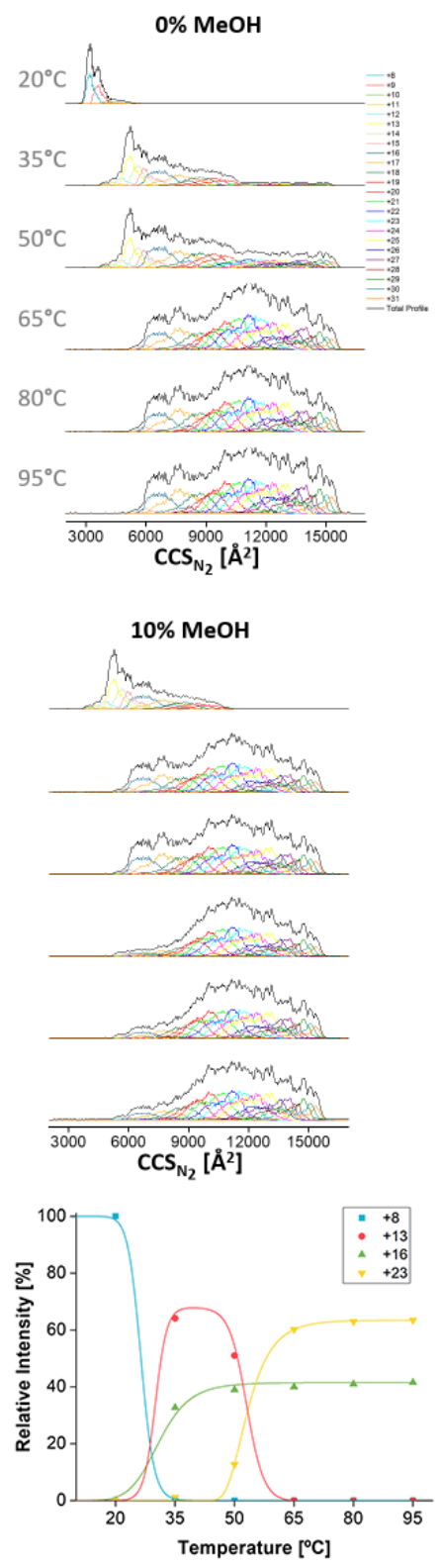
Figure 7.4 Digestion of DehI with CPDY. A: $\mathrm{CCS}_{\mathrm{N} 2}$ distribution of the observed fragments as a function of the mass of each fragment. Orange circles: undigested protein; Green triangles: fragments with residues removed exclusively from the C-terminal; Red triangles: fragments with residues removed exclusively from the N-terminal; Blue rhomboids: fragments with residues removed from the both termini; Black circles: fragments where a structural collapse is observed; Black stars and grey line: fragments where the knot is preserved after the digestion. B: Sequence coverage by the digestion. C: IMS profiles of the fragments that preserve the knot.
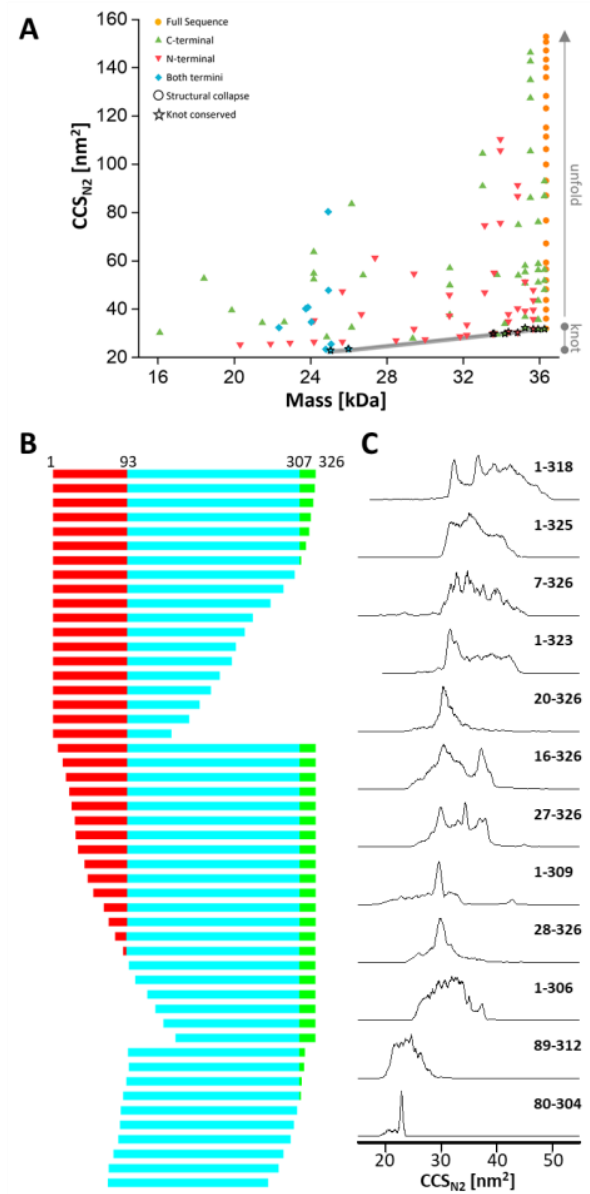


\section{LIST OF REFERENCES}

(1) Virnau, P.; Mirny, L. A.; Kardar, M. PLoS Comput Biol 2006, 2, e122.

(2) and, F. T.; Kamitori, S. 1996.

(3) Yeates, T. O.; Norcross, T. S.; King, N. P. Current opinion in chemical biology 2007, $11,595-603$.

(4) Faísca, P. F. In Computational and structural biotechnology journal, 2015, pp 459-468.

(5) Bolinger, D.; Sulkowska, J. I.; Hsu, H. P.; Mirny, L. A.; Kardar, M.; Onuchic, J. N.; Virnau, P. PLoS Comput Biol 2014, 6, e1000731.

(6) Castellanos, A.; Benigni, P.; Hernandez, D. R.; DeBord, J. D.; Ridgeway, M. E.; Park, M. A.; Fernandez-Lima, F. Anal Methods 2014, 6, 9328-9332.

(7) Benigni, P.; Sandoval, K.; Thompson, C. J.; Ridgeway, M. E.; Park, M. A.; Gardinali, P.; Fernandez-Lima, F. Environ Sci Technol 2017, 51, 5978-5988.

(8) Schenk, E. R.; Mendez, V.; Landrum, J. T.; Ridgeway, M. E.; Park, M. A.; FernandezLima, F. Anal Chem 2014, 86, 2019-2024.

(9) Molano-Arevalo, J. C.; Hernandez, D. R.; Gonzalez, W. G.; Miksovska, J.; Ridgeway, M. E.; Park, M. A.; Fernandez-Lima, F. Anal Chem 2014, 86, 10223-10230.

(10) McKenzie-Coe, A.; DeBord, J. D.; Ridgeway, M.; Park, M.; Eiceman, G.; FernandezLima, F. Analyst 2015, 140, 5692-5699.

(11) Garabedian, A.; Butcher, D.; Lippens, J. L.; Miksovska, J.; Chapagain, P. P.; Fabris, D.; Ridgeway, M. E.; Park, M. A.; Fernandez-Lima, F. Phys Chem Chem Phys 2016, 18, 26691-26702.

(12) Ridgeway, M. E.; Silveira, J. A.; Meier, J. E.; Park, M. A. Analyst 2015, 140, 69646972.

(13) Silveira, J. A.; Ridgeway, M. E.; Park, M. A. Anal Chem 2014, 86, 5624-5627.

(14) Benigni, P.; Thompson, C. J.; Ridgeway, M. E.; Park, M. A.; Fernandez-Lima, F. Anal Chem 2015, 87, 4321-4325.

(15) Schenk, E. R.; Ridgeway, M. E.; Park, M. A.; Leng, F.; Fernandez-Lima, F. Anal Chem 2014, 86, 1210-1214.

(16) Schenk, E. R.; Almeida, R.; Miksovska, J.; Ridgeway, M. E.; Park, M. A.; FernandezLima, F. J Am Soc Mass Spectrom 2015, 26, 555-563. 
(17) Benigni, P.; Marin, R.; Molano-Arevalo, J. C.; Garabedian, A.; Wolff, J. J.; Ridgeway, M. E.; Park, M. A.; Fernandez-Lima, F. International journal for ion mobility spectrometry : official publication of the International Society for Ion Mobility Spectrometry 2016, 19, 95-104.

(18) Fernandez-Lima, F.; Kaplan, D. A.; Suetering, J.; Park, M. A. International journal for ion mobility spectrometry : official publication of the International Society for Ion Mobility Spectrometry 2011, 14, 93-98.

(19) Fernandez-Lima, F. A.; Kaplan, D. A.; Park, M. A. The Review of scientific instruments 2011, 82, 126106.

(20) Fernandez-Lima, F. A.; Kaplan, D. A.; Park, M. A. Rev. Sci. Instrum. 2011, 82, 126106.

(21) Fernandez-Lima, F.; Kaplan, D. A.; Suetering, J.; Park, M. A. Int. J. Ion Mobil. Spectrom. 2011, 14, 93-98.

(22) Hernandez, D. R.; Debord, J. D.; Ridgeway, M. E.; Kaplan, D. A.; Park, M. A.; Fernandez-Lima, F. Analyst 2014, 139, 1913-1921.

(23) Molano-Arevalo, J. C.; Hernandez, D. R.; Gonzalez, W. G.; Miksovska, J.; Ridgeway, M. E.; Park, M. A.; Fernandez-Lima, F. Anal. Chem. 2014, 86, 10223-10230.

(24) Schenk, E. R.; Ridgeway, M. E.; Park, M. A.; Leng, F.; Fernandez-Lima, F. Anal. Chem. 2014, 86, 1210-1214.

(25) McDaniel, E. W.; Mason, E. A. Mobility and diffusion of ions in gases; John Wiley and Sons, Inc., New York: New York, 1973, p 381.

(26) United States, 1999.

(27) Lim, K.; Zhang, H.; Tempczyk, A.; Krajewski, W.; Bonander, N.; Toedt, J.; Howard, A.; Eisenstein, E.; Herzberg, O. Proteins 2003, 51, 56-67.

(28) Larriba, C.; Hogan, C. J., Jr. J. Phys. Chem. A 2013, 117, 3887-3901.

(29) Larriba, C.; Hogan, C. J. J. Comput. Phys. 2013, 251, 344-363. 


\section{APPENDICES}




\section{SUPPORTING INFORMATION FOR CHAPTER 2. FLAVIN ADENINE DINUCLEOTIDE STRUCTURAL MOTIFS: FROM SOLUTION TO GAS-PHASE}

Figure S2.1. FAD total MS spectrum.

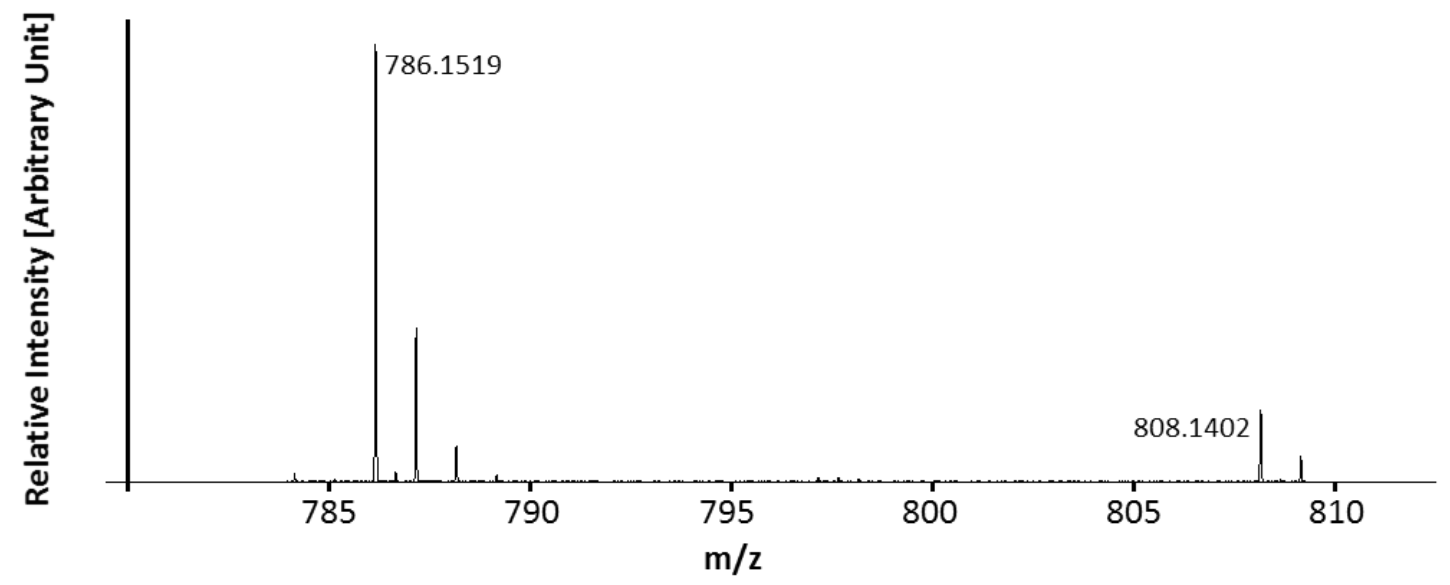


Figure S2.2. FAD "open” conformation theoretical structure. Optimization of the structure was done at level B3LYP/6-31G(d,P) and CCS was calculate using mobcal.

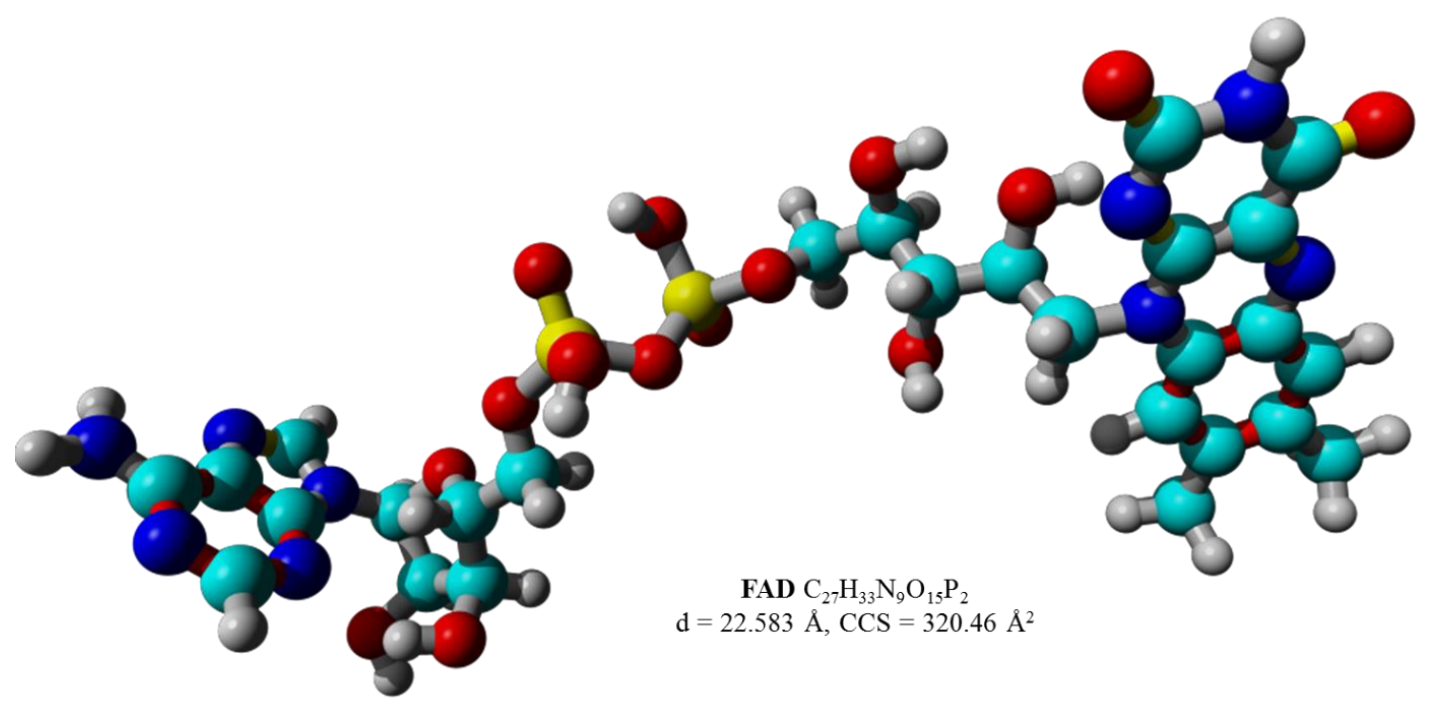


SUPPORTING INFORMATION FOR CHAPTER 3: INSIGHTS FROM ION MOBILITY - MASS SPECTROMETRY, INFRARED SPECTROSCOPY, AND MOLECULAR DYNAMICS ON NICOTINAMIDE ADENINE DINUCLEOTIDE STRUCTURAL DYNAMICS: NAD ${ }^{+} V S$ NADH

Figure S3.1. Scheme of the TIMS cell

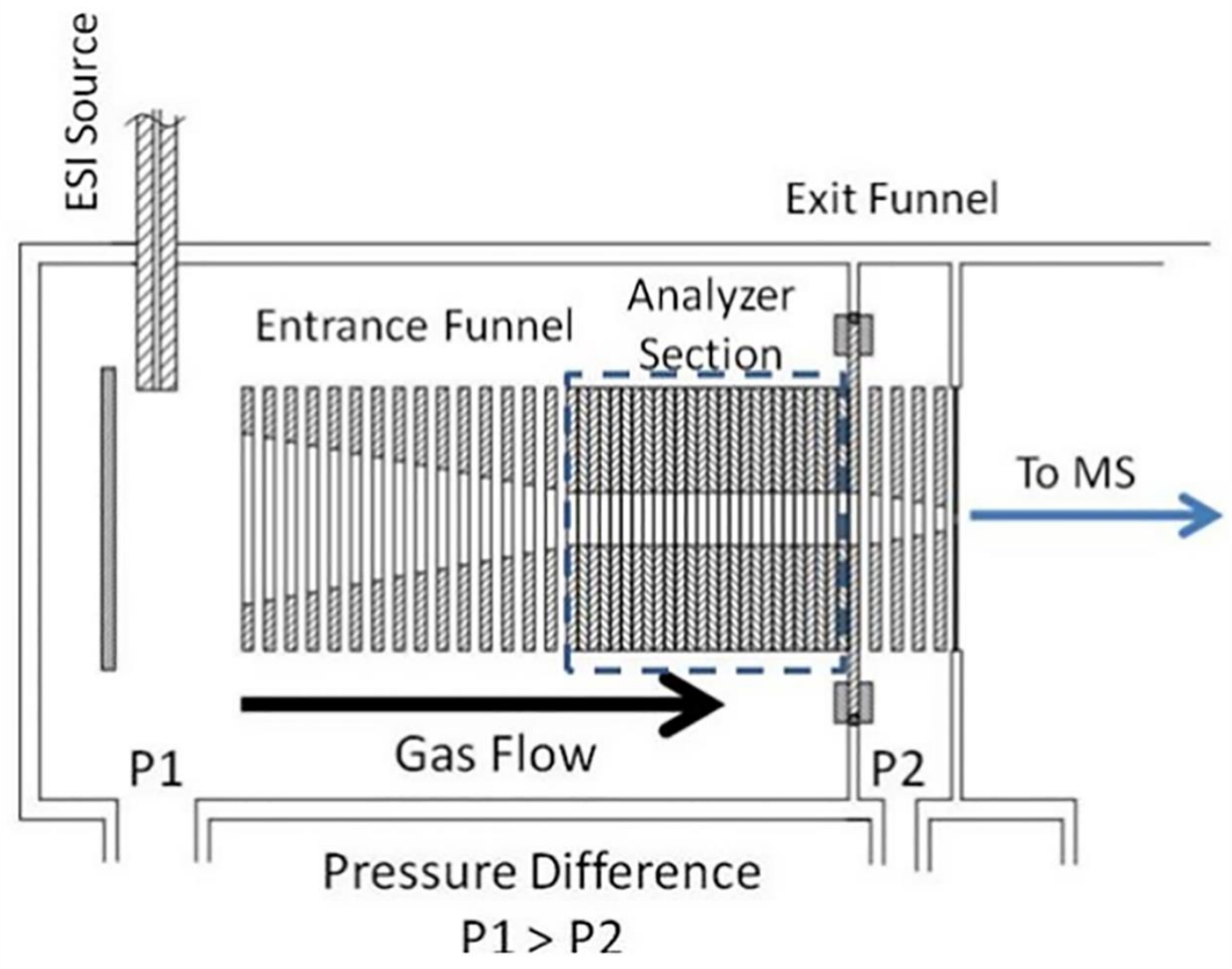


Figure S3.2. Lowest energy candidate structures proposed for NADH IMS band A obtained at DFT/B3LYP/6-31G*using Jaguar software.
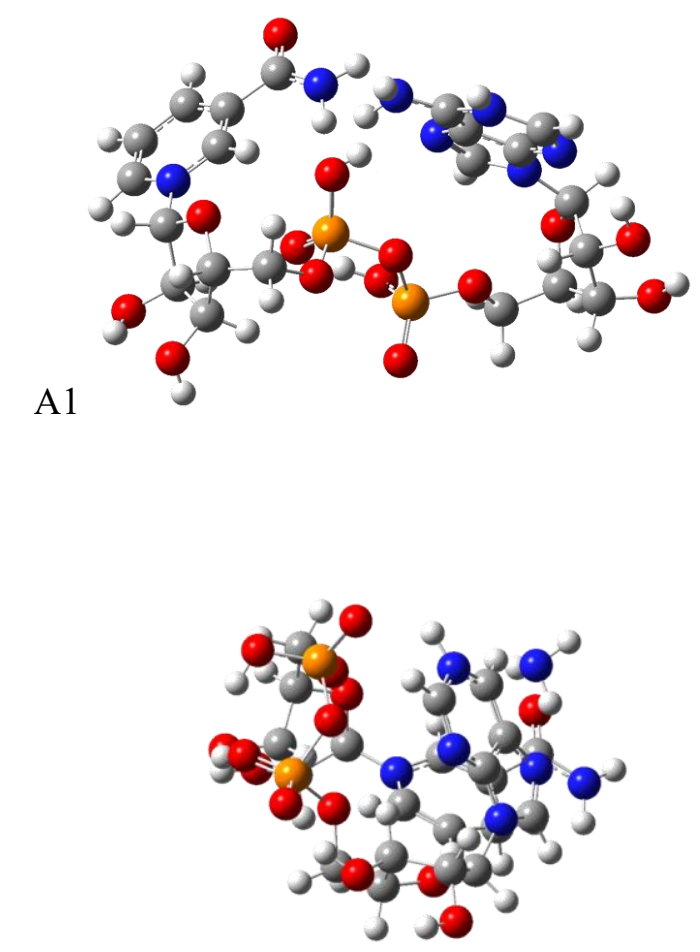

A2

A3

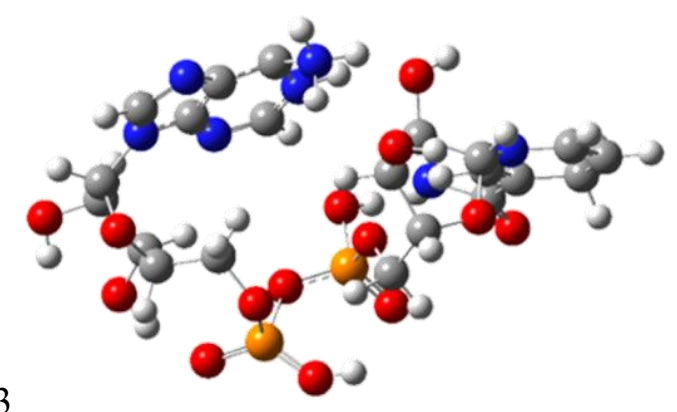


Figure S3.3. Lowest energy candidate structures proposed for NADH IMS band B obtained at DFT/B3LYP/6${ }_{31} G^{*}$ using Jaguar software.

B1
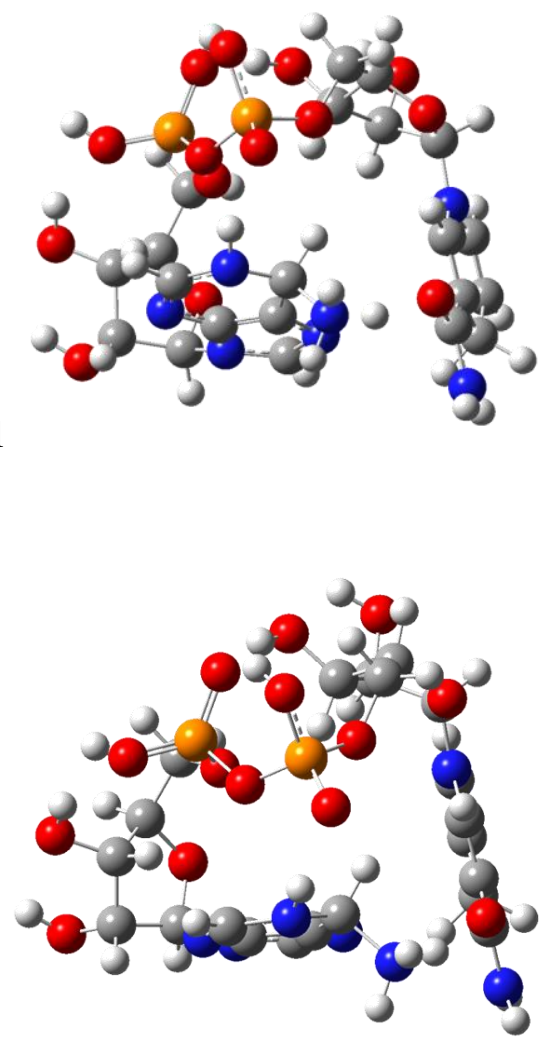

B2

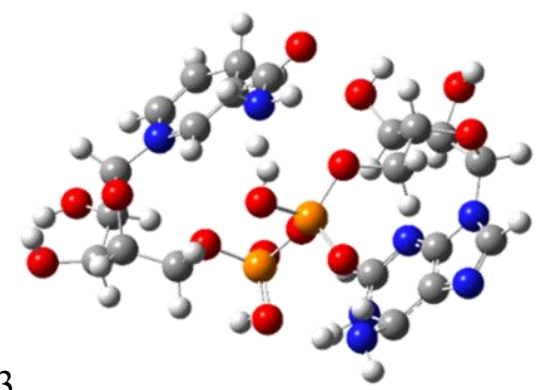

B3 
Figure S3.4. Lowest energy candidate structures proposed for NADH IMS band C obtained at DFT/B3LYP/6-31G*using Jaguar software.
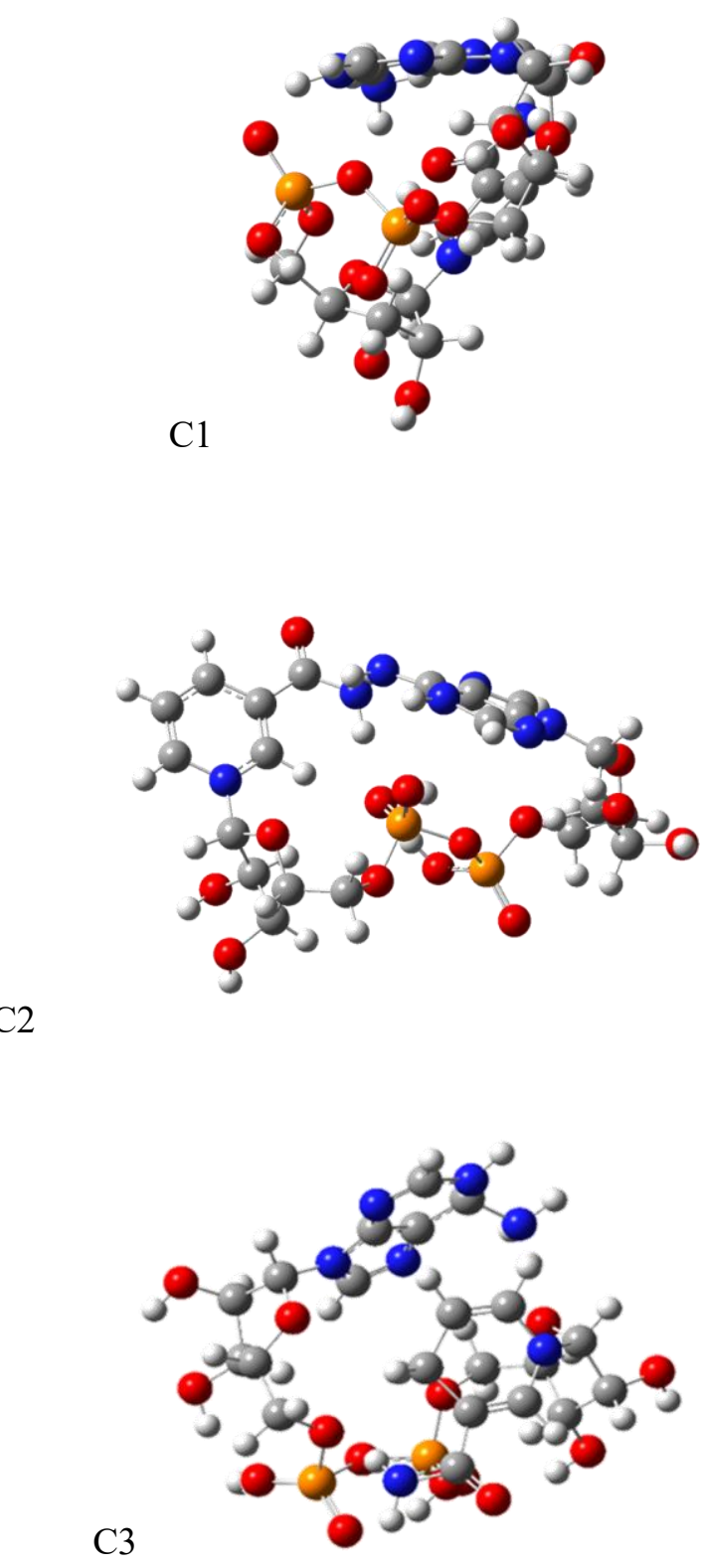
Figure S3.5. Lowest energy candidate structures proposed for NADH IMS band D obtained at DFT/B3LYP/6-31G*using Jaguar software.

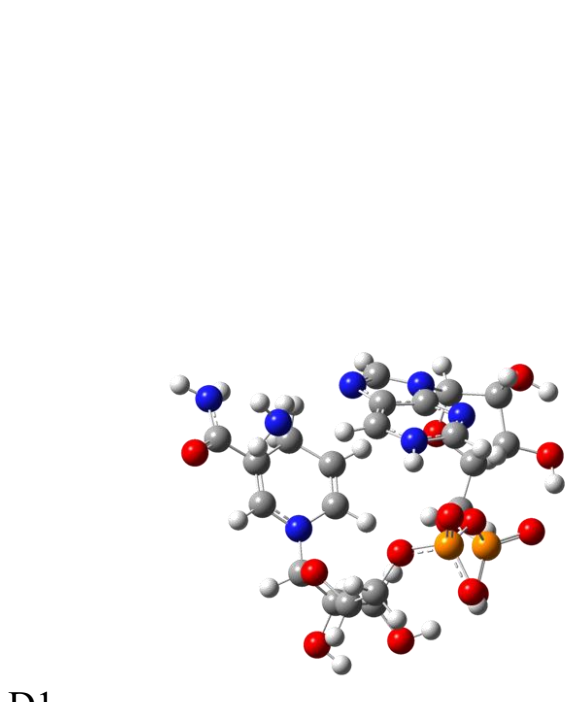

D1
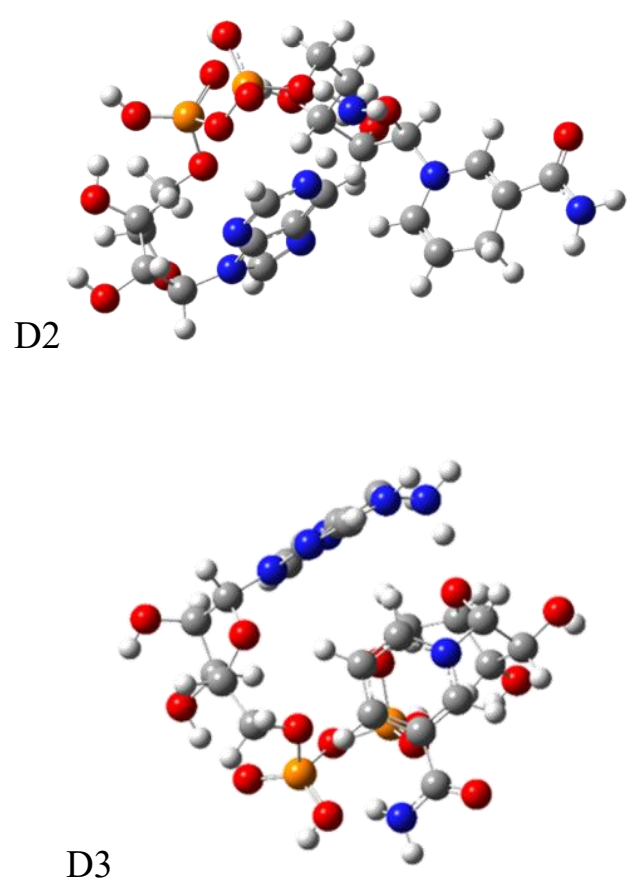
Figure S3.6. Lowest energy candidate structures proposed for NADH IMS band G obtained at DFT/B3LYP/6-31G*using Jaguar software.
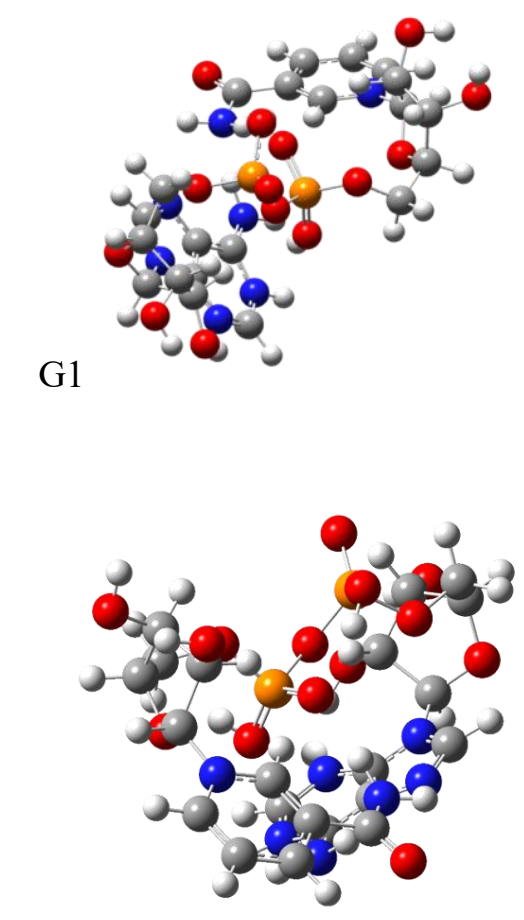

G2

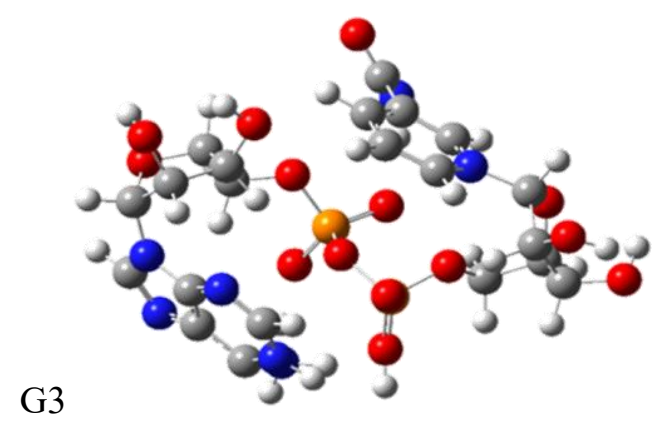


Figure S3.7. Lowest energy candidate structures proposed for NADH IMS band $\mathrm{H}$ obtained at DFT/B3LYP/6-31G*using Jaguar software.

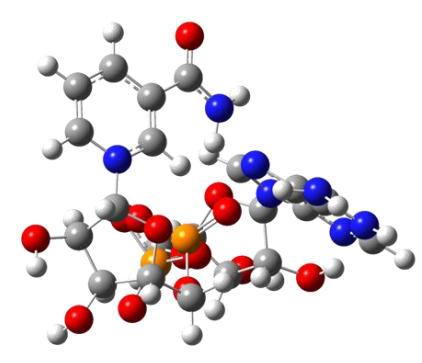

$\mathrm{H} 1$

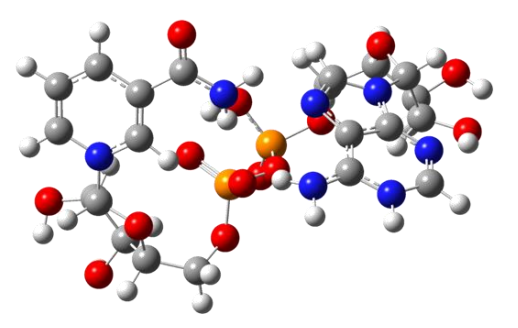

$\mathrm{H} 2$

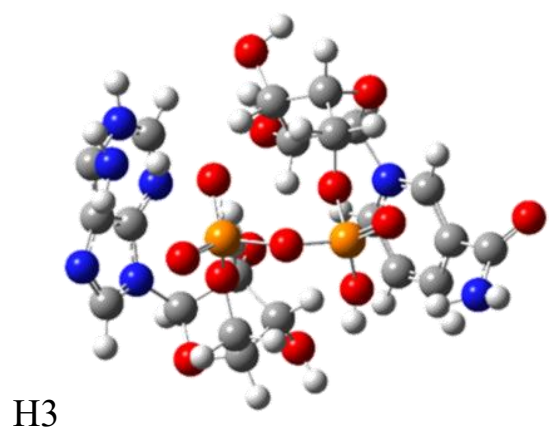


Figure S3.8. Theoretical IR spectra of the three, lowest energy structure proposed based on the IMS bands for $\mathrm{NAD}^{+}$and NADH obtained at DFT/B3LYP/6-31G* using Jaguar software.
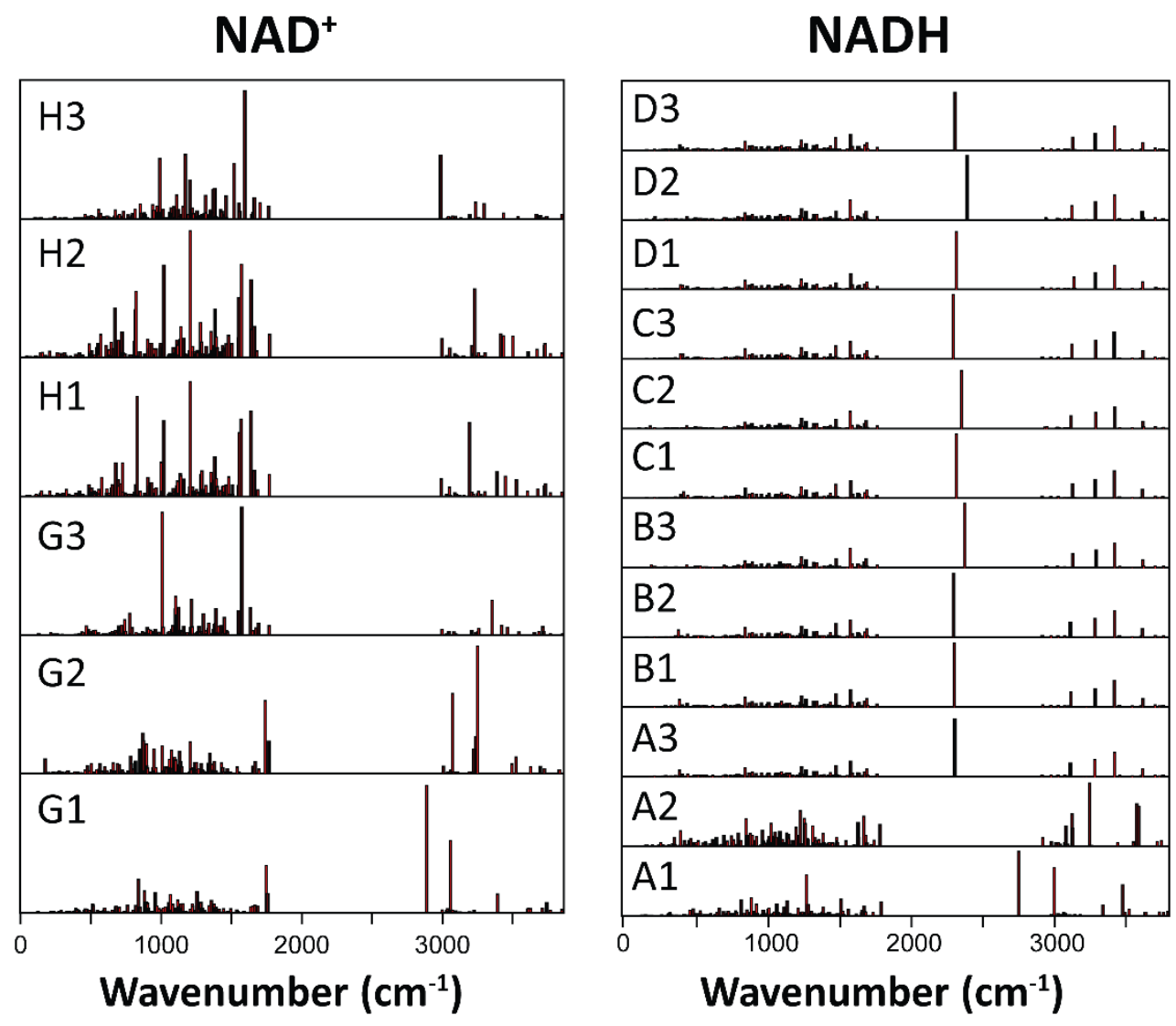
Figure S3.9. Nomenclature utilized for atoms present in the structure of NADH and NAD ${ }^{+}$.
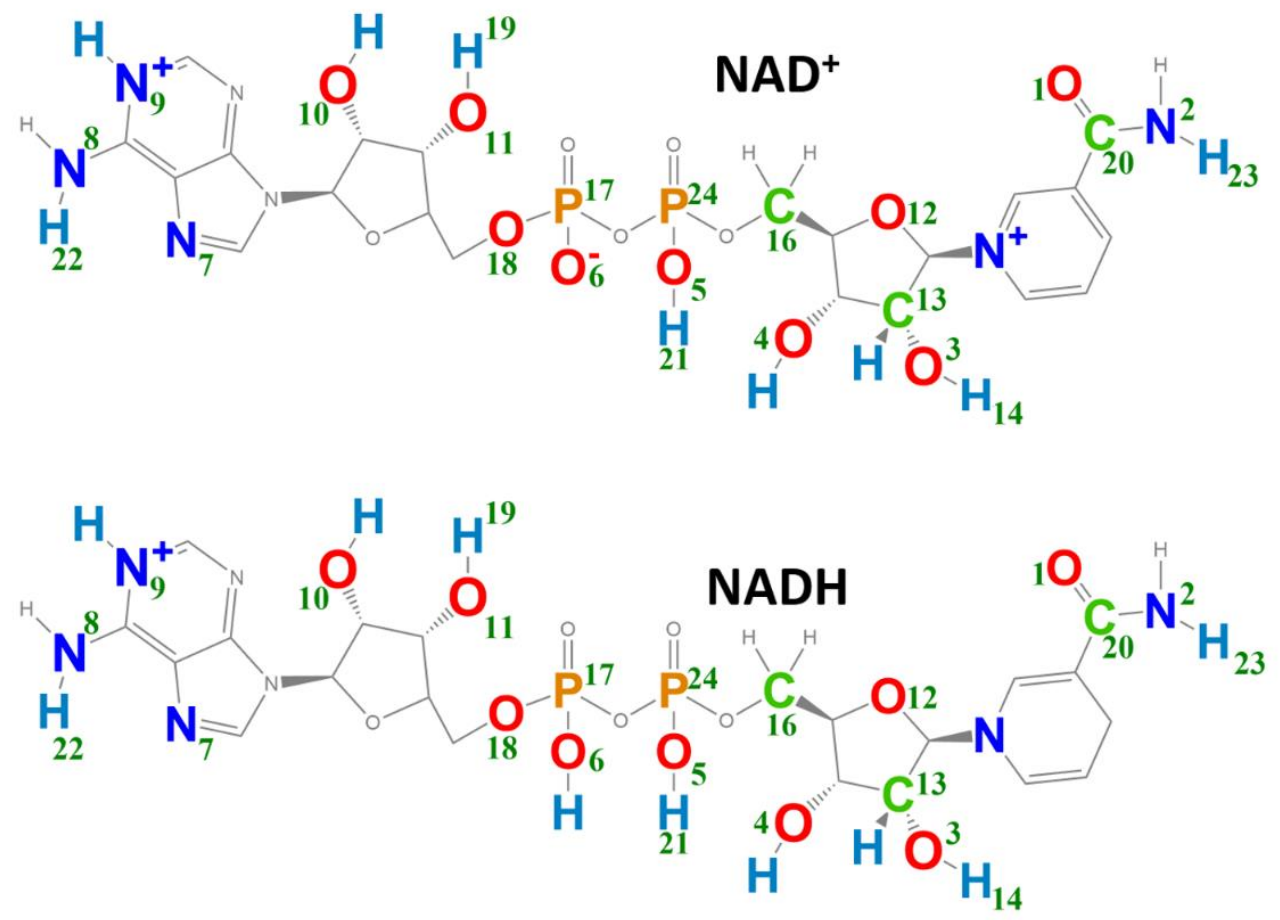
Figure S3.10. Characteristic and common intramolecular interactions of NADH and NAD ${ }^{+}$.

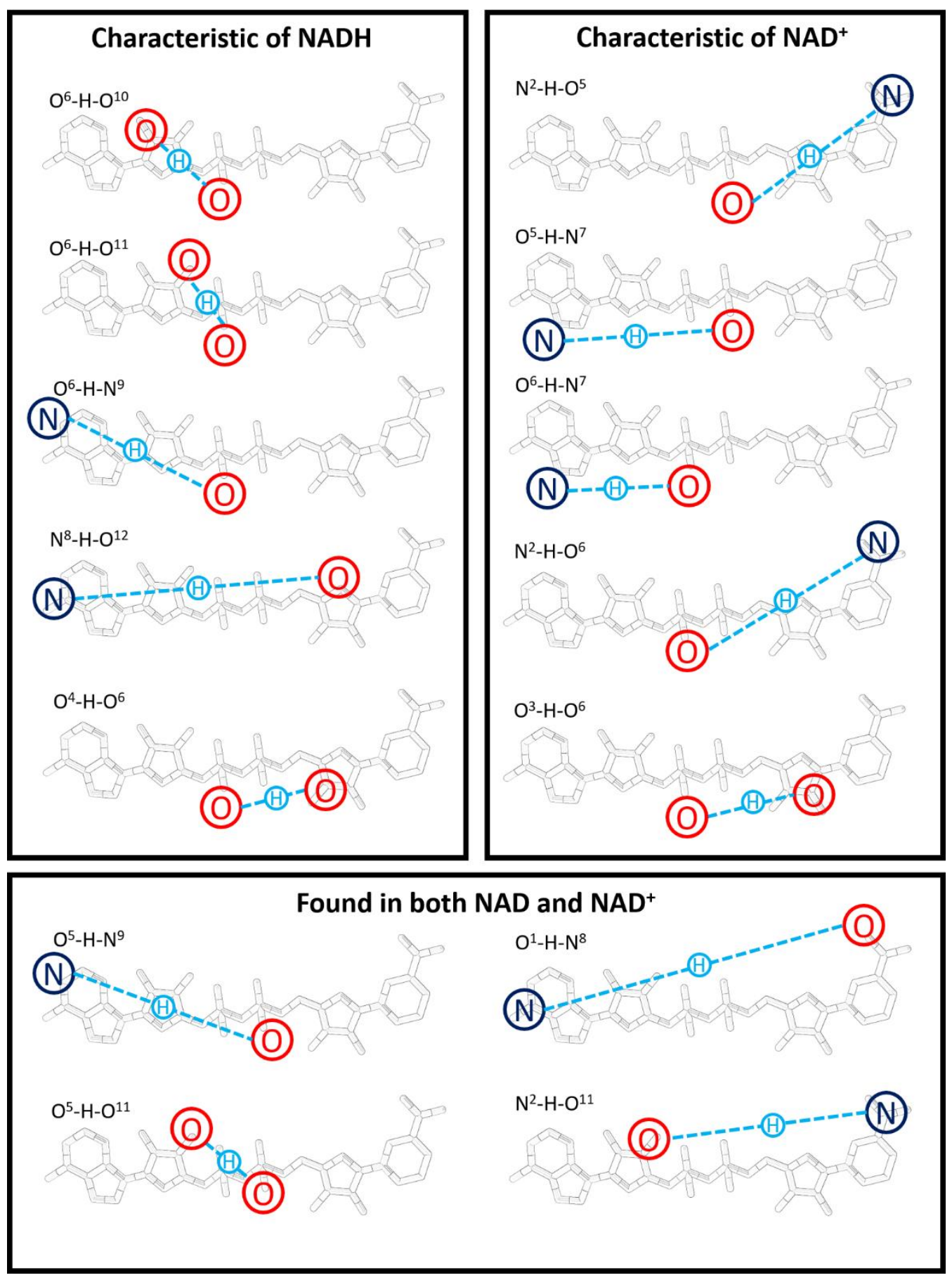


Figure S3.11. Intramolecular interactions of $\mathrm{NADH}$ and $\mathrm{NAD}^{+}$sorted by the main conformational families.
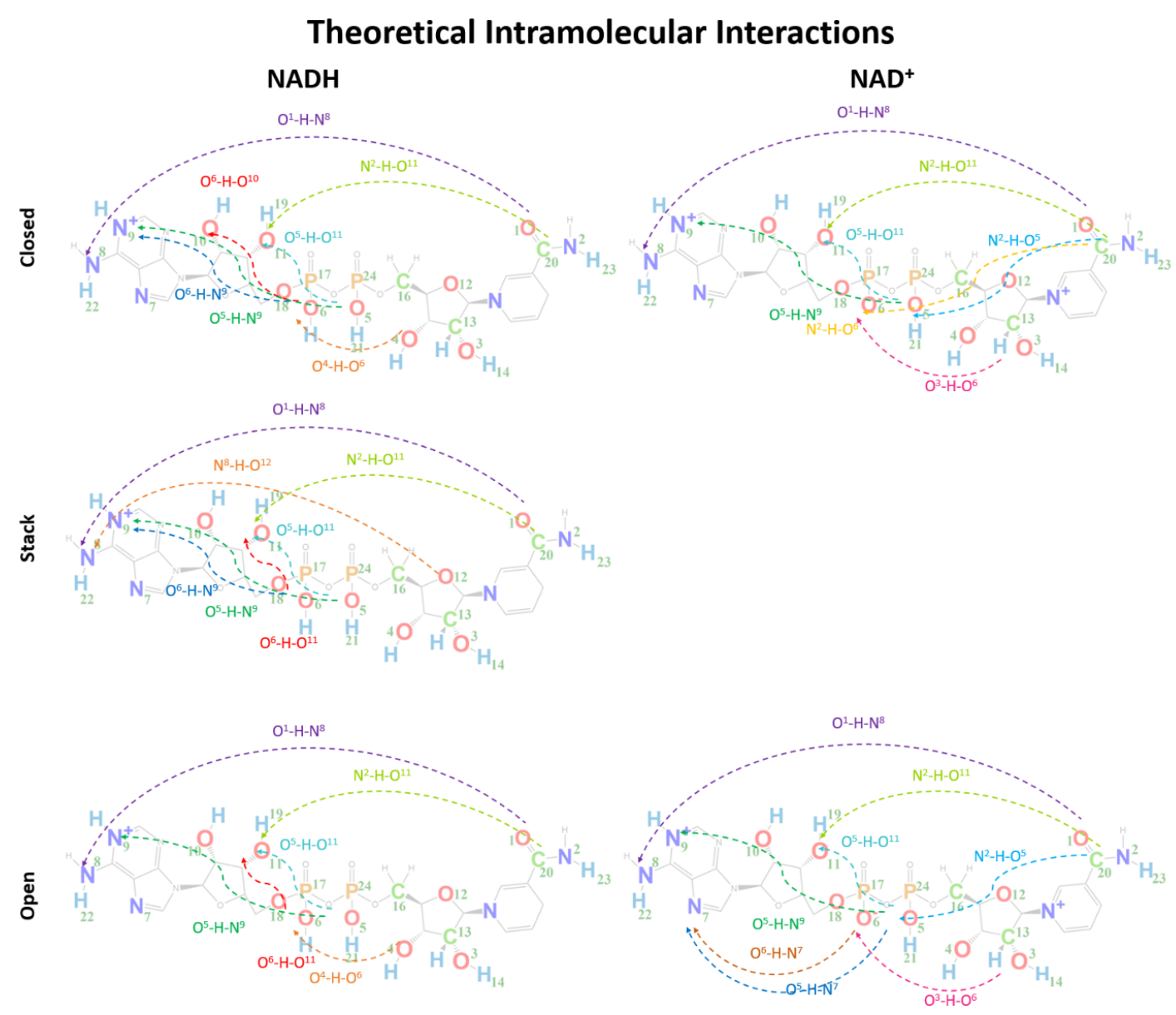
Figure S3.12. Lowest energy candidate structures proposed for the sodiated form of NADH and $\mathrm{NAD}^{+}$based on the IMS bands obtained at DFT/B3LYP/6-31G*using Jaguar software.
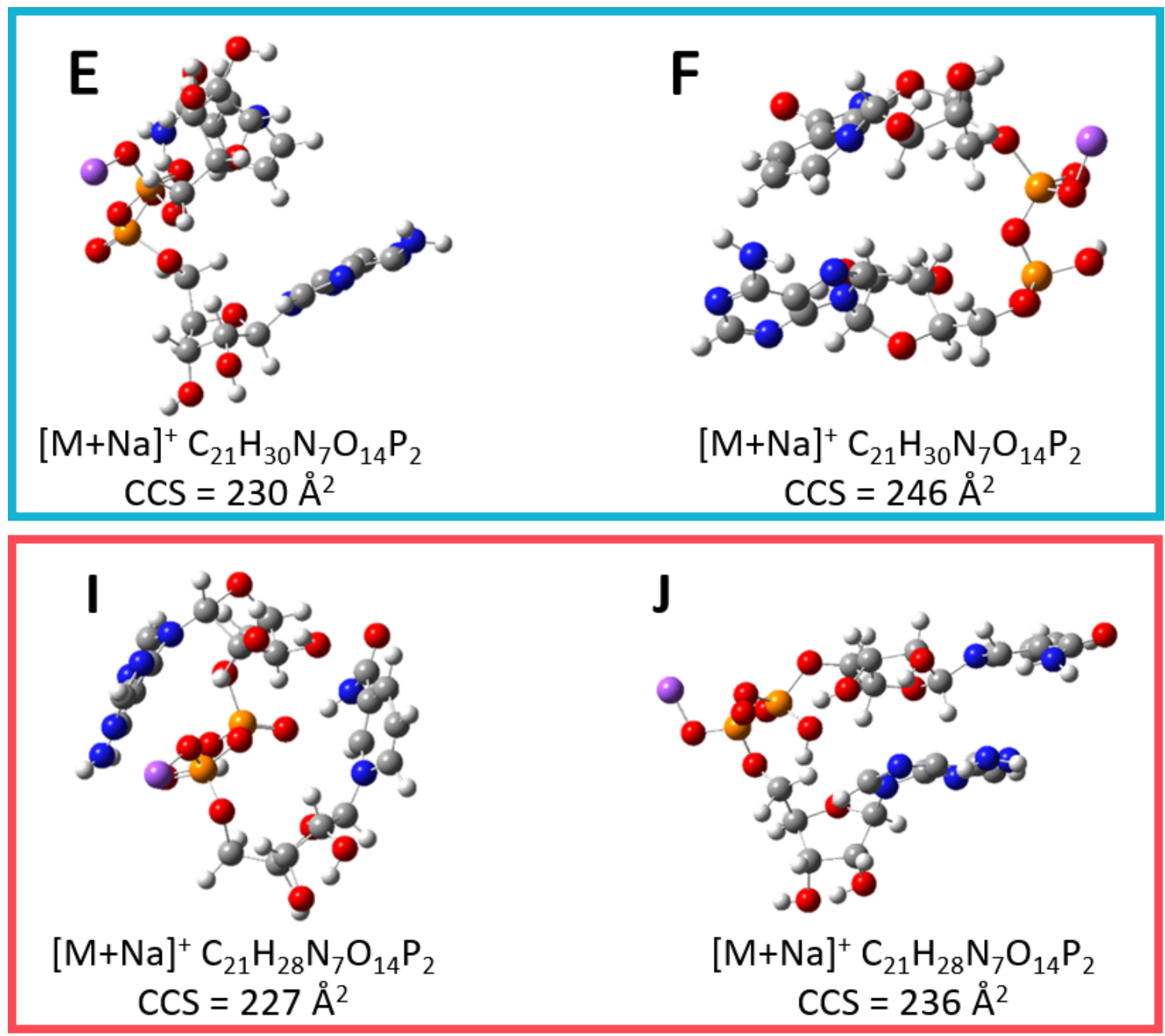
Table S3.1. Fluorescence decay parameters recovered for NADH in solution as a function of $\mathrm{EtOH}$ or $\mathrm{MeOH}$ content. $\mathrm{NAD}^{+}$is not fluorescent. The phase shift as a function the frequency was modeled using a double exponential model, where alpha $(\alpha)$ and tau $(\tau)$ are the pre-exponential factor and lifetime, respectively. The double exponential model was utilized assuming a two-state model: more "close" and more "open" conformations. The longer the lifetime, the more open the conformation.

\begin{tabular}{llllll}
\hline & $\alpha_{0}$ & $\tau_{0}(\mathrm{~ns})$ & $\alpha_{1}$ & $\tau_{1}(\mathrm{~ns})$ & $\begin{array}{l}\tau_{\text {average }} \\
(\mathrm{ns})\end{array}$ \\
\hline $\begin{array}{l}10 \mathrm{mM} \mathrm{NH} 4 \mathrm{Ace} \mathrm{pH} \\
8.0\end{array}$ & 0.86 & 0.3 & 0.14 & 0.94 & 0.50 \\
\hline$+10 \% \mathrm{MeOH}$ & 0.86 & 0.14 & 0.52 \\
\hline$+30 \% \mathrm{MeOH}$ & 0.83 & 0.17 & 0.55 \\
\hline$+50 \% \mathrm{MeOH}$ & 0.81 & 0.19 & 0.57 \\
\hline$+70 \% \mathrm{MeOH}$ & 0.76 & 0.24 & 0.62 \\
\hline$+10 \% \mathrm{EtOH}$ & 0.83 & & 0.17 & 0.58 \\
\hline$+30 \% \mathrm{EtOH}$ & 0.80 & 0.20 & 0.64 \\
\hline$+50 \% \mathrm{EtOH}$ & 0.73 & 0.27 & 0.68 \\
\hline$+70 \% \mathrm{EtOH}$ & 0.68 & 0.32 & \\
\hline
\end{tabular}


Table S3.2. Experimental and theoretical ion-neutral collision cross section $\left(\mathrm{CCS}_{\mathrm{N} 2}, \AA^{2}\right)$ for the sodiated $\mathrm{NAD}^{+}$and NADH forms.

\begin{tabular}{|c|c|c|c|c|}
\hline & \multicolumn{2}{|c|}{$\begin{array}{l}\text { B3LYP/6-31G(d,p) with } \\
\text { charges }\end{array}$} \\
\hline Ion & & $\begin{array}{l}\text { Experimental } \\
\mathrm{CCS}_{\mathrm{N} 2}\left[\AA^{2}\right]\end{array}$ & $\begin{array}{l}\text { Theoretical } \\
\text { CCS } S_{\mathrm{N} 2} \text { IMos } \\
\text { TM }\left[\AA^{2}\right]\end{array}$ & $\begin{array}{l}\text { Relative } \\
\text { energy } \\
{[\mathrm{kcal} / \mathrm{mol}]}\end{array}$ \\
\hline \multirow{2}{*}{$\begin{array}{l}{\left[\mathrm{M}_{\mathrm{NADH}}+\mathrm{Na}\right]^{+}} \\
\mathrm{C}_{21} \mathrm{H}_{30} \mathrm{~N}_{7} \mathrm{O}_{14} \mathrm{P}_{2} \\
m / z 688.12 \\
\Delta \mathrm{m} / \mathrm{m} 0.3 \mathrm{ppm}\end{array}$} & E & 234 & 235.1 & 0 \\
\hline & F & 246 & 246.4 & 2.19 \\
\hline \multirow{2}{*}{$\begin{array}{l}{ }^{\left[\mathrm{M}_{\mathrm{NAD}+}+\right.} \\
\mathrm{Na}]^{+} \\
\mathrm{C}_{21} \mathrm{H}_{29} \mathrm{~N}_{7} \mathrm{O}_{14} \mathrm{P}_{2} \\
m / z 686.09 \\
\Delta \mathrm{m} / \mathrm{m} 0.7 \mathrm{ppm}\end{array}$} & I & 227 & 229.5 & 0 \\
\hline & $\mathrm{J}$ & 236 & 237.3 & 3.95 \\
\hline
\end{tabular}


Table S3.3. Experimental and theoretical ion-neutral collision cross section $\left(\mathrm{CCS}_{\mathrm{N} 2}, \AA^{2}\right)$ and vibrational frequencies $\left(\mathrm{cm}^{-1}\right)$ for the protonated NADH form.

\begin{tabular}{|c|c|c|c|c|c|c|c|c|c|c|c|c|c|c|c|}
\hline & & $\begin{array}{l}\text { B3L } \\
31 \mathrm{G} \\
\text { with }\end{array}$ & & & & & IR B & Bands & & prop & & & actio & ns fo & \\
\hline Ion & $\begin{array}{l}\text { Exp } \\
\cdot \\
\text { CC } \\
\mathrm{S} \\
{\left[\AA^{2}\right.} \\
]\end{array}$ & $\begin{array}{l}\text { The } \\
\text { or. } \\
\text { CCS } \\
\text { IMo } \\
\text { s } \\
\text { TM } \\
{\left[\AA^{2}\right]}\end{array}$ & $\begin{array}{l}\text { Rel. } \\
\text { ener } \\
\mathrm{g} \\
{[\mathrm{kca}} \\
1 / \mathrm{mo} \\
1]\end{array}$ & 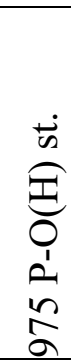 & $\begin{array}{l}\dot{0} \\
\text { 总 } \\
0 \\
\stackrel{1}{0} \\
0 \\
0 \\
0\end{array}$ & 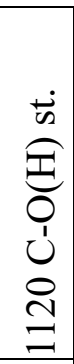 & $\begin{array}{l}0 \\
0 \\
0 \\
1 \\
0 \\
0 \\
心 \\
\end{array}$ & 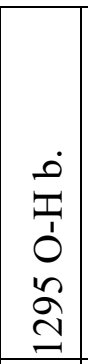 & 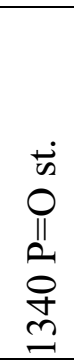 & 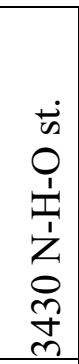 & 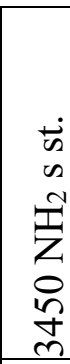 & $\begin{array}{l}\dot{\Delta} \\
0 \\
0 \\
1 \\
1 \\
0 \\
0 \\
0 \\
0 \\
n \\
m\end{array}$ & $\begin{array}{l}\dot{\omega} \\
\tilde{n} \\
\tilde{N} \\
\vec{Z} \\
z \\
o \\
0 \\
n \\
m\end{array}$ & 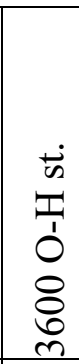 & 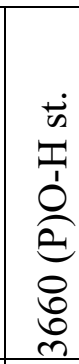 \\
\hline \multirow{5}{*}{$\begin{array}{l}{\left[\mathrm{M}_{\mathrm{NADH}^{+}}\right.} \\
\mathrm{H}]^{+} \\
\mathrm{C}_{21} \mathrm{H}_{30} \mathrm{~N}_{7} \\
\mathrm{O}_{14} \mathrm{P}_{2} \\
\mathrm{~m} / \mathrm{z} \\
666.13 \\
\Delta \mathrm{m} / \mathrm{m} \\
1.5 \mathrm{ppm}\end{array}$} & \multirow{3}{*}{ A 234} & $\begin{array}{l}234 . \\
6\end{array}$ & $\begin{array}{l}13.9 \\
5\end{array}$ & $\begin{array}{l}\mathrm{P}^{2} \\
{ }_{-} \\
\mathrm{O} \\
5\end{array}$ & $\begin{array}{l}\mathrm{P}^{2} \\
4- \\
\mathrm{O} \\
5- \\
\mathrm{H} \\
21\end{array}$ & $\begin{array}{l}\mathrm{C}^{1} \\
3- \\
\mathrm{O} \\
3- \\
\mathrm{H} \\
14\end{array}$ & & $\begin{array}{l}\mathrm{O} \\
11 \\
\mathrm{H} \\
19\end{array}$ & & & $\begin{array}{l}\mathrm{N} \\
2 / \\
\mathrm{N} \\
8\end{array}$ & $\begin{array}{l}\mathrm{O} \\
5- \\
\mathrm{H} \\
21 \\
\mathrm{O} \\
11\end{array}$ & $\begin{array}{l}\mathrm{N} \\
2 / \\
\mathrm{N} \\
8\end{array}$ & $\begin{array}{l}\mathrm{O} \\
3- \\
\mathrm{H} \\
14\end{array}$ & $\begin{array}{l}\mathrm{P}^{2} \\
4- \\
\mathrm{O}_{-} \\
5- \\
\mathrm{H} \\
21\end{array}$ \\
\hline & & $\begin{array}{l}234 . \\
3\end{array}$ & $\begin{array}{l}14.3 \\
7\end{array}$ & $\begin{array}{l}\mathrm{P}^{2} \\
4- \\
\mathrm{O} \\
5\end{array}$ & & $\begin{array}{l}\mathrm{C}^{1} \\
3- \\
\mathrm{O} \\
3- \\
\mathrm{H} \\
14\end{array}$ & $\begin{array}{l}\mathrm{P}^{1} \\
7- \\
\mathrm{O} \\
18\end{array}$ & $\begin{array}{l}\mathrm{O} \\
11 \\
\mathrm{H} \\
19\end{array}$ & & $\begin{array}{l}\mathrm{N} \\
2 \_ \\
\mathrm{H} \\
23 \_ \\
\mathrm{O} \\
11\end{array}$ & $\begin{array}{l}\mathrm{N} \\
2 / \\
\mathrm{N} \\
8\end{array}$ & $\begin{array}{l}\mathrm{O} \\
5- \\
\mathrm{H}_{-} \\
21 \\
\mathrm{O} \\
11\end{array}$ & $\begin{array}{l}\mathrm{N} \\
2 / \\
\mathrm{N} \\
8\end{array}$ & $\begin{array}{l}\mathrm{O} \\
3- \\
\mathrm{H}_{-} \\
14\end{array}$ & $\begin{array}{l}\mathrm{P}^{2} \\
4- \\
\mathrm{O} \\
5- \\
\mathrm{H}_{21}\end{array}$ \\
\hline & & $\begin{array}{l}234 . \\
8\end{array}$ & $\begin{array}{l}14.8 \\
3\end{array}$ & $\begin{array}{l}\mathrm{P}^{2} \\
4- \\
\mathrm{O} \\
6\end{array}$ & $\begin{array}{l}\mathrm{P}^{2} \\
4- \\
\mathrm{O} \\
5- \\
\mathrm{H} \\
21\end{array}$ & $\begin{array}{l}\mathrm{C}^{1} \\
3_{-} \\
\mathrm{O} \\
3_{-} \\
\mathrm{H}^{2} \\
14\end{array}$ & & $\begin{array}{l}\mathrm{O} \\
11 \\
\mathrm{H} \\
19\end{array}$ & $\begin{array}{l}\mathrm{P}^{1} \\
7- \\
\mathrm{O}_{6} \\
\end{array}$ & & $\begin{array}{l}\mathrm{N} \\
2 / \\
\mathrm{N} \\
8\end{array}$ & $\begin{array}{l}\mathrm{O} \\
5- \\
\mathrm{H} \\
21 \_ \\
\mathrm{O} \\
11\end{array}$ & $\begin{array}{l}\mathrm{N} \\
2 / \\
\mathrm{N} \\
8\end{array}$ & $\begin{array}{l}\mathrm{O} \\
3- \\
\mathrm{H} \\
14\end{array}$ & $\begin{array}{l}\mathrm{P}^{2} \\
4- \\
\mathrm{O} \\
5- \\
\mathrm{H} \\
21\end{array}$ \\
\hline & \multirow[t]{2}{*}{ B 239} & $\begin{array}{l}239 . \\
4\end{array}$ & 0 & $\begin{array}{l}\mathrm{P}^{2} \\
4- \\
\mathrm{O} \\
5\end{array}$ & & $\begin{array}{l}\mathrm{C}^{1} \\
3- \\
\mathrm{O}^{-} \\
3_{-} \\
\mathrm{H} \\
14\end{array}$ & $\begin{array}{l}\mathrm{P}^{1} \\
7- \\
\mathrm{O} \\
18\end{array}$ & $\begin{array}{l}\mathrm{O} \\
11 \\
\mathrm{H} \\
19\end{array}$ & $\begin{array}{l}\mathrm{P}^{1} \\
7- \\
\mathrm{O}^{-} \\
6\end{array}$ & $\begin{array}{l}\mathrm{N} \\
2- \\
\mathrm{H} \\
23 \\
\mathrm{O} \\
11\end{array}$ & $\begin{array}{l}\mathrm{N} \\
2 / \\
\mathrm{N} \\
8\end{array}$ & $\begin{array}{l}\mathrm{O} \\
5- \\
\mathrm{H}_{2} \\
21 \\
\mathrm{O} \\
11\end{array}$ & $\begin{array}{l}\mathrm{N} \\
2 / \\
\mathrm{N} \\
8\end{array}$ & $\begin{array}{l}\mathrm{O} \\
3- \\
\mathrm{H}_{-} \\
14\end{array}$ & $\begin{array}{l}\mathrm{P}^{2} \\
4- \\
\mathrm{O}_{-} \\
5- \\
\mathrm{H}_{2} \\
21\end{array}$ \\
\hline & & $\begin{array}{l}239 . \\
4\end{array}$ & 8.49 & & $\begin{array}{l}\mathrm{P}^{2} \\
{ }_{-} \\
\mathrm{O}\end{array}$ & $\begin{array}{l}\mathrm{C}^{1} \\
3- \\
\mathrm{O}\end{array}$ & & $\begin{array}{l}\mathrm{O} \\
11\end{array}$ & $\begin{array}{l}\mathrm{P}^{1} \\
7-\end{array}$ & & $\begin{array}{l}\mathrm{N} \\
2 /\end{array}$ & $\begin{array}{l}\mathrm{O} \\
5- \\
\mathrm{H}\end{array}$ & $\begin{array}{l}\mathrm{N} \\
2 /\end{array}$ & $\begin{array}{l}\mathrm{O} \\
{ }_{3}\end{array}$ & $\begin{array}{l}P^{2} \\
4- \\
O\end{array}$ \\
\hline
\end{tabular}




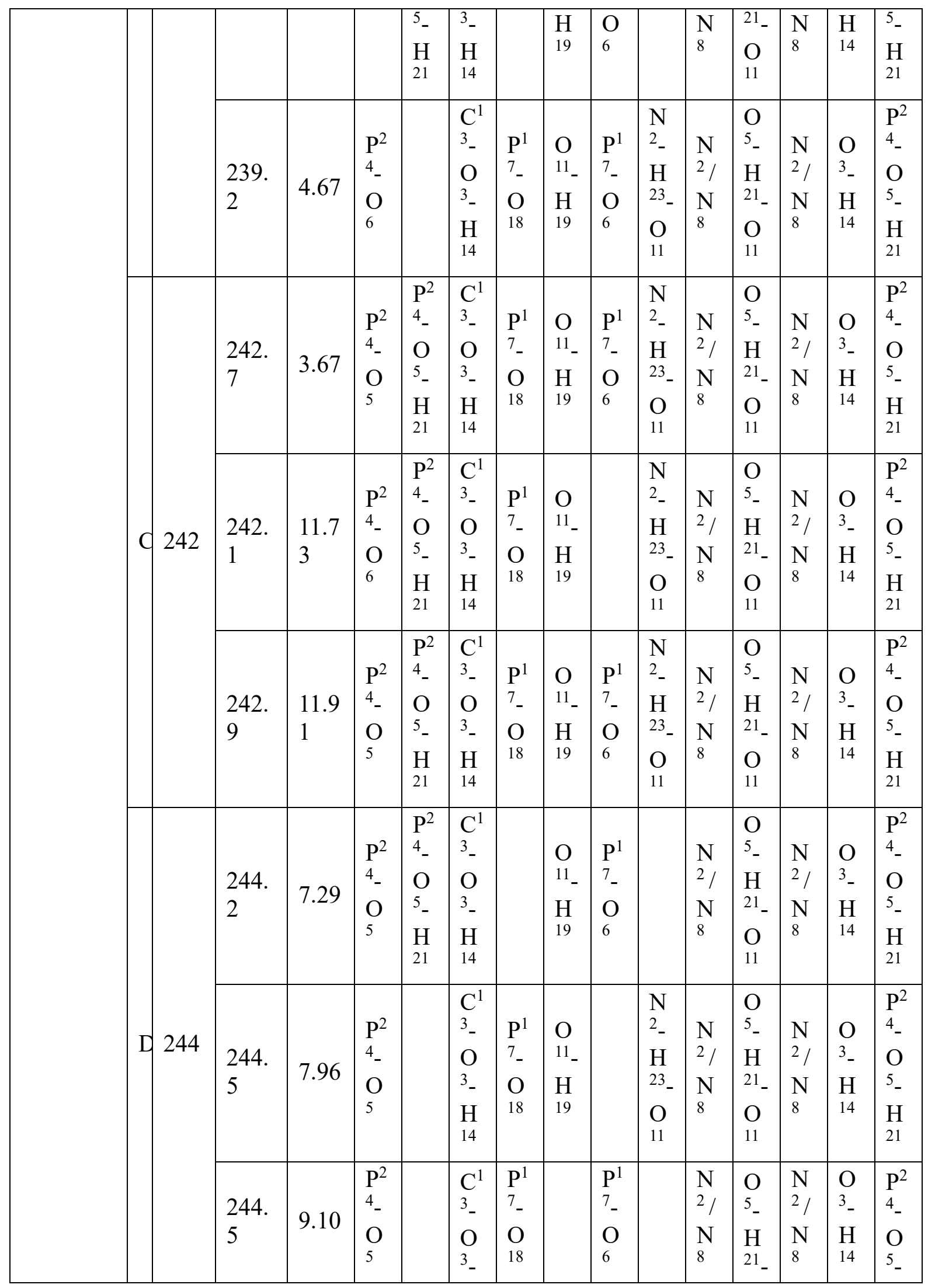




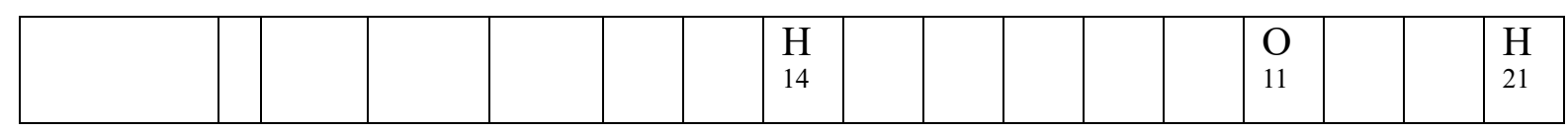


Table S3.4. Experimental and theoretical ion-neutral collision cross section $\left(\mathrm{CCS}_{\mathrm{N} 2}, \AA^{2}\right)$ and vibrational frequencies $\left(\mathrm{cm}^{-1}\right)$ for the protonated $\mathrm{NAD}^{+}$form.

\begin{tabular}{|c|c|c|c|c|c|c|c|c|c|c|c|c|c|c|c|c|}
\hline & & $\begin{array}{l}\text { B3L) } \\
31 \mathrm{G}( \\
\text { with } \\
\text { charg }\end{array}$ & & & & & 1 IR & Ban & ds a & ad $\mathrm{p}$ & ropo & sed i & inter & actiol & ns fo & \\
\hline Ion & \begin{tabular}{|l} 
Exp \\
$\cdot$ \\
CC \\
$\mathrm{S}$ \\
{$\left[\AA^{2}\right.$} \\
]
\end{tabular} & $\begin{array}{l}\text { The } \\
\text { or. } \\
\text { CCS } \\
\text { IMo } \\
\text { s } \\
\text { TM } \\
{\left[\AA^{2}\right]}\end{array}$ & $\begin{array}{l}\text { Rel. } \\
\text { ener } \\
\mathrm{g} \\
{[\mathrm{kca}} \\
1 / \mathrm{mo} \\
1]\end{array}$ & 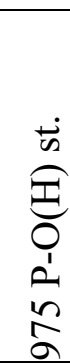 & $\begin{array}{l}\dot{w} \\
0 \\
1 \\
0 \\
0 \\
\vdots \\
0 \\
\vdots \\
\exists\end{array}$ & 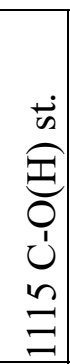 & $\begin{array}{l}0 \\
0 \\
0 \\
1 \\
2 \\
\varrho \\
\Xi\end{array}$ & $\begin{array}{l}\dot{3} \\
\text { İ } \\
0 \\
0 \\
\stackrel{+}{I}\end{array}$ & 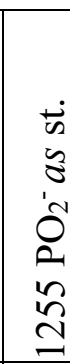 & $\begin{array}{l}\dot{m} \\
0 \\
0 \\
11 \\
0 \\
0 \\
m \\
m\end{array}$ & $\begin{array}{l}\dot{1} \\
0 \\
0 \\
0 \\
0 \\
0 \\
0 \\
0\end{array}$ & $\begin{array}{l}\dot{w} \\
0 \\
0 \\
1 \\
\dot{1} \\
z \\
0 \\
0 \\
m \\
\tilde{m} \\
m\end{array}$ & $\begin{array}{l}\dot{0} \\
0 \\
1 \\
1 \\
1 \\
0 \\
0 \\
0 \\
+ \\
m\end{array}$ & $\mid \begin{array}{c}\dot{n} \\
0 \\
0 \\
1 \\
1 \\
0 \\
0 \\
0 \\
\dot{1} \\
n \\
m\end{array}$ & 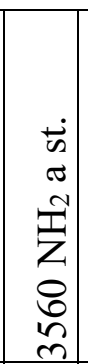 & 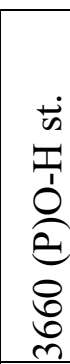 \\
\hline & & $\begin{array}{l}232 . \\
8\end{array}$ & 0 & $\begin{array}{l}\mathrm{P} \\
24 \\
- \\
\mathrm{O} \\
5\end{array}$ & $\begin{array}{l}\mathrm{P} \\
24\end{array}$ & $\begin{array}{l}\mathrm{C} \\
13 \\
- \\
\mathrm{O} \\
3- \\
\mathrm{H} \\
14\end{array}$ & & $\begin{array}{l}\mathrm{O} \\
3- \\
\mathrm{H} \\
14\end{array}$ & $\begin{array}{l}\mathrm{P} \\
17\end{array}$ & & $\begin{array}{l}\mathrm{C} \\
20 \\
- \\
\mathrm{O} \\
1\end{array}$ & $\begin{array}{l}\mathrm{N} \\
2- \\
\mathrm{H} \\
23 \\
- \\
\mathrm{O} \\
11\end{array}$ & \begin{tabular}{|l|}
$\mathrm{O}$ \\
$3-$ \\
$\mathrm{H}$ \\
14 \\
- \\
$\mathrm{O}$ \\
6
\end{tabular} & \begin{tabular}{|l|}
$\mathrm{O}$ \\
5 \\
- \\
$\mathrm{H}$ \\
21 \\
- \\
$\mathrm{O}$ \\
11
\end{tabular} & $\begin{array}{l}\mathrm{N} \\
2 / \\
\mathrm{N} \\
8\end{array}$ & $\begin{array}{l}\mathrm{P} \\
24 \\
- \\
\mathrm{O} \\
5- \\
\mathrm{H} \\
21\end{array}$ \\
\hline $\begin{array}{l}{\left[\mathrm{M}_{\mathrm{NAD}^{+}+}+\right.} \\
\mathrm{H}]^{+} \\
\mathrm{C}_{21} \mathrm{H}_{28} \mathrm{~N}_{7} \\
\mathrm{O}_{144} \mathrm{P}_{2} \\
\mathrm{~m} / z\end{array}$ & d 230 & $\begin{array}{l}232 . \\
6\end{array}$ & 7.05 & $\begin{array}{l}\mathrm{P} \\
24 \\
- \\
\mathrm{O} \\
5\end{array}$ & $\begin{array}{l}\mathrm{P} \\
24\end{array}$ & $\begin{array}{l}\mathrm{C} \\
13 \\
- \\
\mathrm{O} \\
3- \\
\mathrm{H} \\
14\end{array}$ & & $\begin{array}{l}\mathrm{O} \\
3- \\
\mathrm{H} \\
14\end{array}$ & $\begin{array}{l}\mathrm{P} \\
17\end{array}$ & & $\begin{array}{l}\mathrm{C} \\
20 \\
- \\
\mathrm{O} \\
1\end{array}$ & $\begin{array}{l}\mathrm{N} \\
2- \\
\mathrm{H} \\
23 \\
- \\
\mathrm{O} \\
11\end{array}$ & \begin{tabular}{|l|}
$\mathrm{O}$ \\
$3-$ \\
$\mathrm{H}$ \\
14 \\
- \\
$\mathrm{O}$ \\
6
\end{tabular} & \begin{tabular}{|l}
$\mathrm{O}$ \\
$5-$ \\
$\mathrm{H}$ \\
21 \\
- \\
$\mathrm{O}$ \\
11
\end{tabular} & $\begin{array}{l}\mathrm{N} \\
2 / \\
\mathrm{N} \\
8\end{array}$ & $\begin{array}{l}\mathrm{P} \\
24 \\
- \\
\mathrm{O} \\
5- \\
\mathrm{H} \\
21\end{array}$ \\
\hline $\begin{array}{l}664.11 \\
\Delta \mathrm{m} / \mathrm{m} \\
1.9 \mathrm{ppm}\end{array}$ & & $\begin{array}{l}232 . \\
3\end{array}$ & 6.84 & $\begin{array}{l}\mathrm{P} \\
24 \\
- \\
\mathrm{O} \\
5\end{array}$ & $\begin{array}{l}\mathrm{P} \\
24\end{array}$ & $\begin{array}{l}\mathrm{C} \\
13 \\
- \\
\mathrm{O} \\
3- \\
\mathrm{H} \\
14\end{array}$ & & $\begin{array}{l}\mathrm{O} \\
3- \\
\mathrm{H} \\
14\end{array}$ & $\begin{array}{l}\mathrm{P} \\
17\end{array}$ & & $\begin{array}{l}\mathrm{C} \\
20 \\
- \\
\mathrm{O} \\
1\end{array}$ & & $\begin{array}{l}\mathrm{O} \\
3- \\
\mathrm{H} \\
14 \\
- \\
\mathrm{O} \\
6\end{array}$ & \begin{tabular}{|l|}
$\mathrm{O}$ \\
$5-$ \\
$\mathrm{H}$ \\
21 \\
- \\
$\mathrm{O}$ \\
11
\end{tabular} & $\begin{array}{l}\mathrm{N} \\
2 / \\
\mathrm{N} \\
8\end{array}$ & \begin{tabular}{|l}
$\mathrm{P}$ \\
24 \\
- \\
$\mathrm{O}$ \\
$5-$ \\
$\mathrm{H}$ \\
21
\end{tabular} \\
\hline & Н 246 & $\begin{array}{l}246 . \\
8\end{array}$ & 5.84 & $\begin{array}{l}\mathrm{P} \\
24 \\
- \\
\mathrm{O} \\
5\end{array}$ & $\begin{array}{l}\mathrm{P} \\
24\end{array}$ & $\begin{array}{l}\mathrm{C} \\
13 \\
- \\
\mathrm{O} \\
3-\end{array}$ & $\begin{array}{l}\mathrm{P} \\
24 \\
- \\
\mathrm{O} \\
5\end{array}$ & & $\begin{array}{l}\mathrm{P} \\
17\end{array}$ & & $\begin{array}{l}\mathrm{C} \\
20 \\
- \\
\mathrm{O} \\
1\end{array}$ & $\begin{array}{l}\mathrm{N} \\
2- \\
\mathrm{H} \\
23 \\
-\end{array}$ & $\begin{array}{l}\mathrm{O} \\
3- \\
\mathrm{H} \\
14 \\
-\end{array}$ & $\begin{array}{l}\mathrm{O} \\
5- \\
\mathrm{H} \\
21 \\
-\end{array}$ & $\begin{array}{l}\mathrm{N} \\
2 / \\
\mathrm{N} \\
8\end{array}$ & $\begin{array}{l}\mathrm{P} \\
24 \\
- \\
\mathrm{O} \\
5\end{array}$ \\
\hline
\end{tabular}




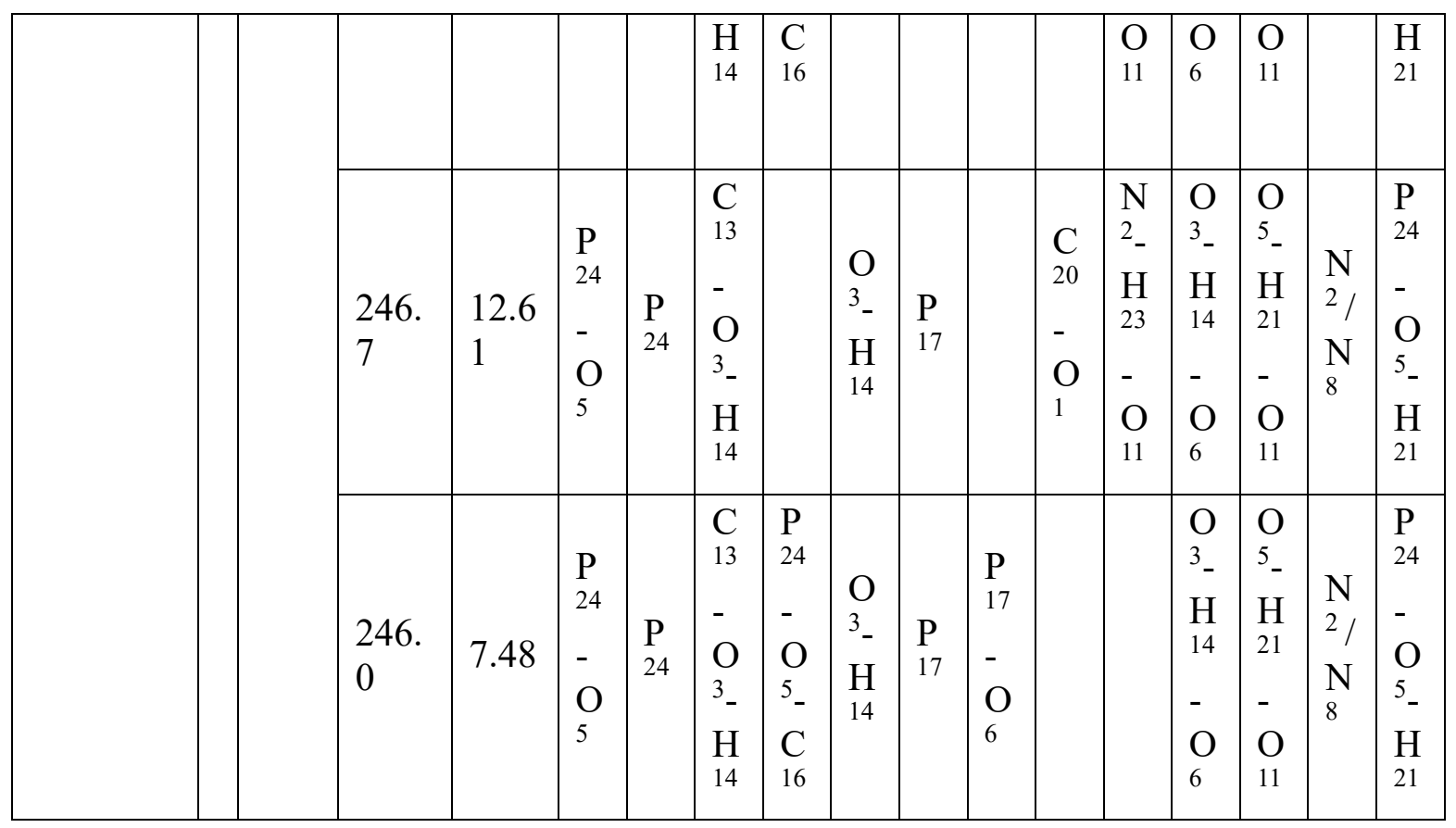


Table S3.5. Theoretical intramolecular interactions of NADH.

\begin{tabular}{|c|c|c|c|c|c|c|c|c|c|c|c|c|c|}
\hline & & & & & \multicolumn{9}{|c|}{ Vibrational Frequencies $\left[\mathrm{cm}^{-1}\right]$} \\
\hline & & & & & \multicolumn{5}{|c|}{ Characteristic of NADH } & \multicolumn{4}{|c|}{ Present in Both } \\
\hline Group & & $\begin{array}{c}\text { Exp. } \\
\text { CCS } \\
\text { N2 } \\
{\left[\AA^{2}\right]}\end{array}$ & $\begin{array}{l}\text { Theor. } \\
\mathrm{CCS}_{\mathrm{N}} \\
2\left[\AA^{2}\right]\end{array}$ & $\begin{array}{c}\text { Rel. } \\
\text { Energ } \\
\mathrm{y} \\
{[\mathrm{kcal} /} \\
\mathrm{mol}]\end{array}$ & $\begin{array}{l}0 \\
0 \\
11 \\
\overbrace{}^{\prime} \\
0^{\prime}\end{array}$ & $\begin{array}{l}= \\
0 \\
\text { 咅 } \\
\text { bे }^{\prime}\end{array}$ & $\begin{array}{l}\text { Zे } \\
\text { 1 } \\
0^{\prime} \\
0\end{array}$ & 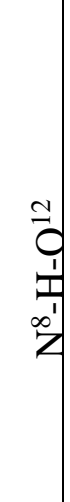 & $\begin{array}{l}0 \\
0 \\
1 \\
0 \\
t^{\prime}\end{array}$ & 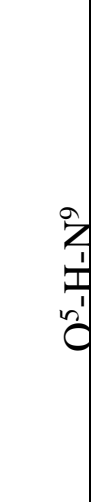 & $\begin{array}{l}Z_{1}^{\infty} \\
\text { i } \\
0 \\
0\end{array}$ & $\begin{array}{l}= \\
0 \\
n^{\prime} \\
n^{\prime}\end{array}$ & 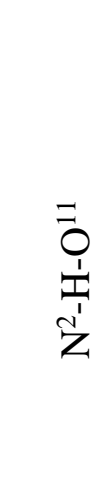 \\
\hline \multirow{3}{*}{ Close } & A & 234 & 234.6 & 13.95 & $\begin{array}{l}36 \\
19\end{array}$ & & $\begin{array}{l}32 \\
87\end{array}$ & & $\begin{array}{l}34 \\
21\end{array}$ & & $\begin{array}{l}33 \\
49\end{array}$ & & \\
\hline & & & 234.3 & 14.37 & $\begin{array}{l}36 \\
08\end{array}$ & & $\begin{array}{l}32 \\
80\end{array}$ & & $\begin{array}{l}34 \\
12\end{array}$ & & $\begin{array}{l}33 \\
39\end{array}$ & $\begin{array}{l}35 \\
94\end{array}$ & $\begin{array}{c}340 \\
9\end{array}$ \\
\hline & & & 234.8 & 14.83 & $\begin{array}{l}36 \\
15\end{array}$ & & $\begin{array}{l}32 \\
98\end{array}$ & & $\begin{array}{l}34 \\
19\end{array}$ & $\begin{array}{l}32 \\
51\end{array}$ & & & \\
\hline
\end{tabular}




\begin{tabular}{|c|c|c|c|c|c|c|c|c|c|c|c|c|}
\hline \multirow{6}{*}{ Stack } & B & 239 & 239.4 & 0 & $\begin{array}{r}35 \\
70\end{array}$ & $\begin{array}{l}32 \\
88\end{array}$ & & & $\begin{array}{l}32 \\
54\end{array}$ & $\begin{array}{l}33 \\
03\end{array}$ & & $\begin{array}{c}341 \\
4\end{array}$ \\
\hline & & & 239.4 & 8.49 & $\begin{array}{l}35 \\
76\end{array}$ & $\begin{array}{l}32 \\
82\end{array}$ & & & $\begin{array}{l}32 \\
51\end{array}$ & $\begin{array}{l}33 \\
06\end{array}$ & $\begin{array}{l}36 \\
03\end{array}$ & \\
\hline & & & 239.2 & 4.67 & $\begin{array}{l}35 \\
68\end{array}$ & $\begin{array}{l}32 \\
83\end{array}$ & & & $\begin{array}{l}32 \\
50\end{array}$ & $\begin{array}{l}33 \\
03\end{array}$ & $\begin{array}{l}35 \\
93\end{array}$ & $\begin{array}{c}341 \\
0\end{array}$ \\
\hline & $\mathrm{C}$ & 242 & 242.7 & 3.67 & $\begin{array}{r}35 \\
77\end{array}$ & & $\begin{array}{l}34 \\
43\end{array}$ & & $\begin{array}{l}32 \\
45\end{array}$ & $\begin{array}{l}33 \\
10\end{array}$ & $\begin{array}{l}35 \\
99\end{array}$ & $\begin{array}{c}342 \\
8\end{array}$ \\
\hline & & & 242.1 & 11.73 & $\begin{array}{r}35 \\
79\end{array}$ & & $\begin{array}{l}34 \\
42\end{array}$ & & $\begin{array}{l}32 \\
49\end{array}$ & $\begin{array}{l}33 \\
02\end{array}$ & & $\begin{array}{c}342 \\
5\end{array}$ \\
\hline & & & 242.9 & 11.91 & $\begin{array}{l}35 \\
83\end{array}$ & & $\begin{array}{l}34 \\
49\end{array}$ & & $\begin{array}{l}32 \\
51\end{array}$ & $\begin{array}{l}33 \\
08\end{array}$ & $\begin{array}{l}35 \\
97\end{array}$ & $\begin{array}{c}343 \\
1\end{array}$ \\
\hline Open & D & 244 & 244.2 & 7.29 & $\begin{array}{l}36 \\
14\end{array}$ & & & $\begin{array}{l}34 \\
25\end{array}$ & $\begin{array}{l}32 \\
54\end{array}$ & $\begin{array}{l}33 \\
09\end{array}$ & & \\
\hline
\end{tabular}




\begin{tabular}{|l|l|l|l|l|l|l|l|l|l|l|l|l|}
\hline & & 244.5 & 7.96 & & 91 & & & 17 & 50 & 01 & & 2 \\
\hline & & 244.5 & 9.10 & & 83 & & & 11 & 59 & 12 & 99 & \\
\hline
\end{tabular}

Table S6. Theoretical intramolecular interactions of $\mathrm{NAD}^{+}$.

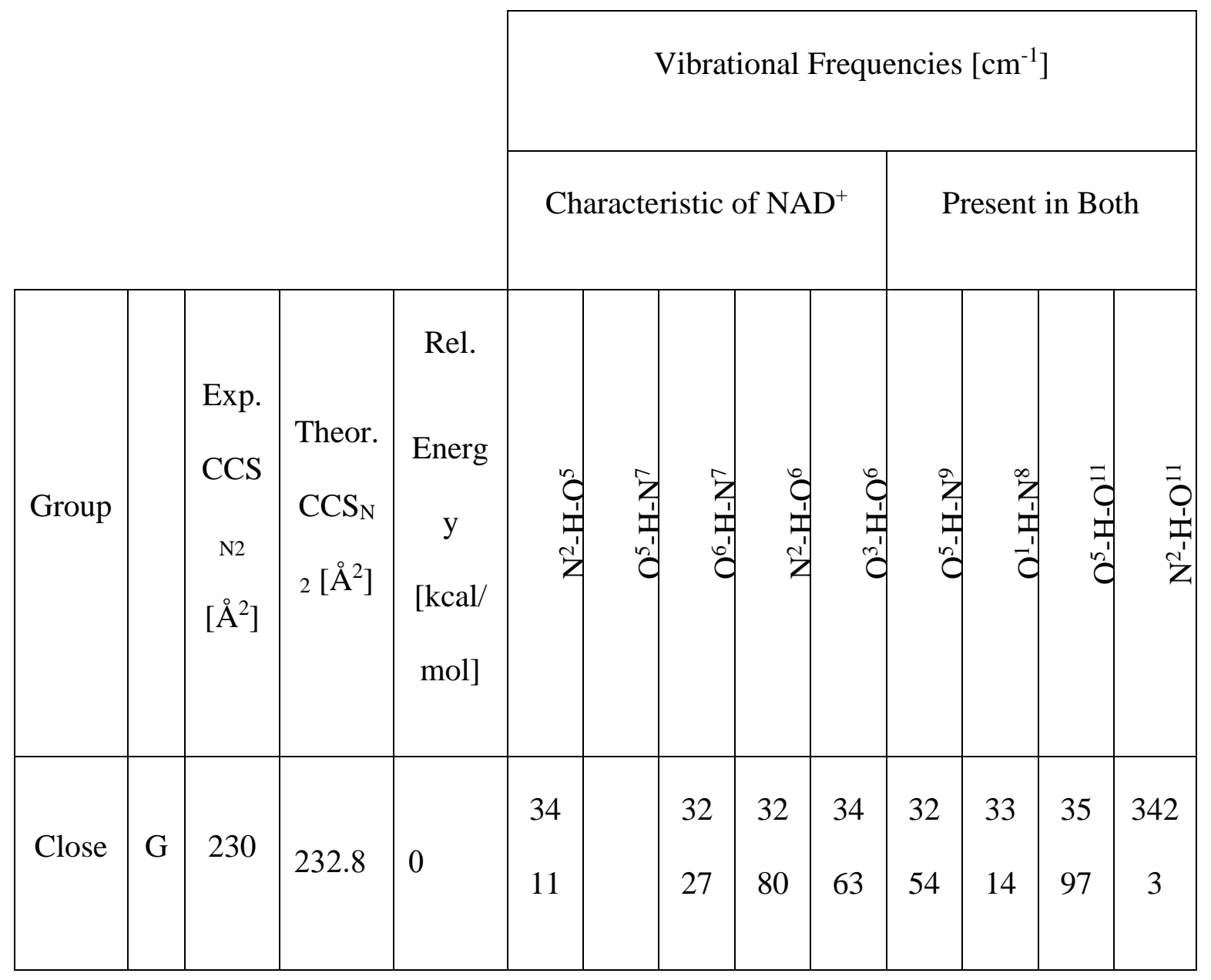




\begin{tabular}{|c|c|c|c|c|c|c|c|c|c|c|c|c|c|}
\hline & & & 232.6 & 7.05 & $\begin{array}{l}34 \\
17\end{array}$ & & $\begin{array}{l}32 \\
32\end{array}$ & $\begin{array}{l}32 \\
79\end{array}$ & $\begin{array}{l}34 \\
67\end{array}$ & $\begin{array}{l}32 \\
50\end{array}$ & $\begin{array}{l}33 \\
14\end{array}$ & $\begin{array}{l}36 \\
01\end{array}$ & $\begin{array}{c}342 \\
1\end{array}$ \\
\hline & & & 232.3 & 6.84 & $\begin{array}{l}34 \\
13\end{array}$ & & $\begin{array}{l}32 \\
29\end{array}$ & $\begin{array}{l}32 \\
93\end{array}$ & $\begin{array}{l}34 \\
68\end{array}$ & $\begin{array}{l}32 \\
53\end{array}$ & $\begin{array}{l}33 \\
22\end{array}$ & & \\
\hline \multirow{3}{*}{ Open } & $\mathrm{H}$ & 246 & 246.8 & 5.84 & $\begin{array}{l}34 \\
22\end{array}$ & $\begin{array}{l}36 \\
17\end{array}$ & $\begin{array}{l}32 \\
21\end{array}$ & & $\begin{array}{l}34 \\
68\end{array}$ & $\begin{array}{l}32 \\
53\end{array}$ & & & $\begin{array}{r}341 \\
2\end{array}$ \\
\hline & & & 246.7 & 12.61 & $\begin{array}{l}34 \\
16\end{array}$ & $\begin{array}{l}36 \\
08\end{array}$ & $\begin{array}{l}32 \\
19\end{array}$ & & $\begin{array}{l}34 \\
62\end{array}$ & $\begin{array}{l}32 \\
47\end{array}$ & $\begin{array}{l}33 \\
09\end{array}$ & $\begin{array}{l}36 \\
02\end{array}$ & $\begin{array}{c}341 \\
9\end{array}$ \\
\hline & & & 246.0 & 7.48 & $\begin{array}{l}34 \\
07\end{array}$ & 14 & $\begin{array}{l}32 \\
27\end{array}$ & & 55 & 55 & & $\begin{array}{l}35 \\
95\end{array}$ & \\
\hline
\end{tabular}


SUPPORTING INFORMATION FOR CHAPTER 4. CHARACTERIZATION OF INTRAMOLECULAR INTERACTIONS OF CYTOCHROME C USING HYDROGEN DEUTERIUM EXCHANGE - TRAPPED ION MOBILITY - MASS SPECTROMETRY AND MOLECULAR DYNAMICS

Figure S4.1. Scheme of the TIMS cell.

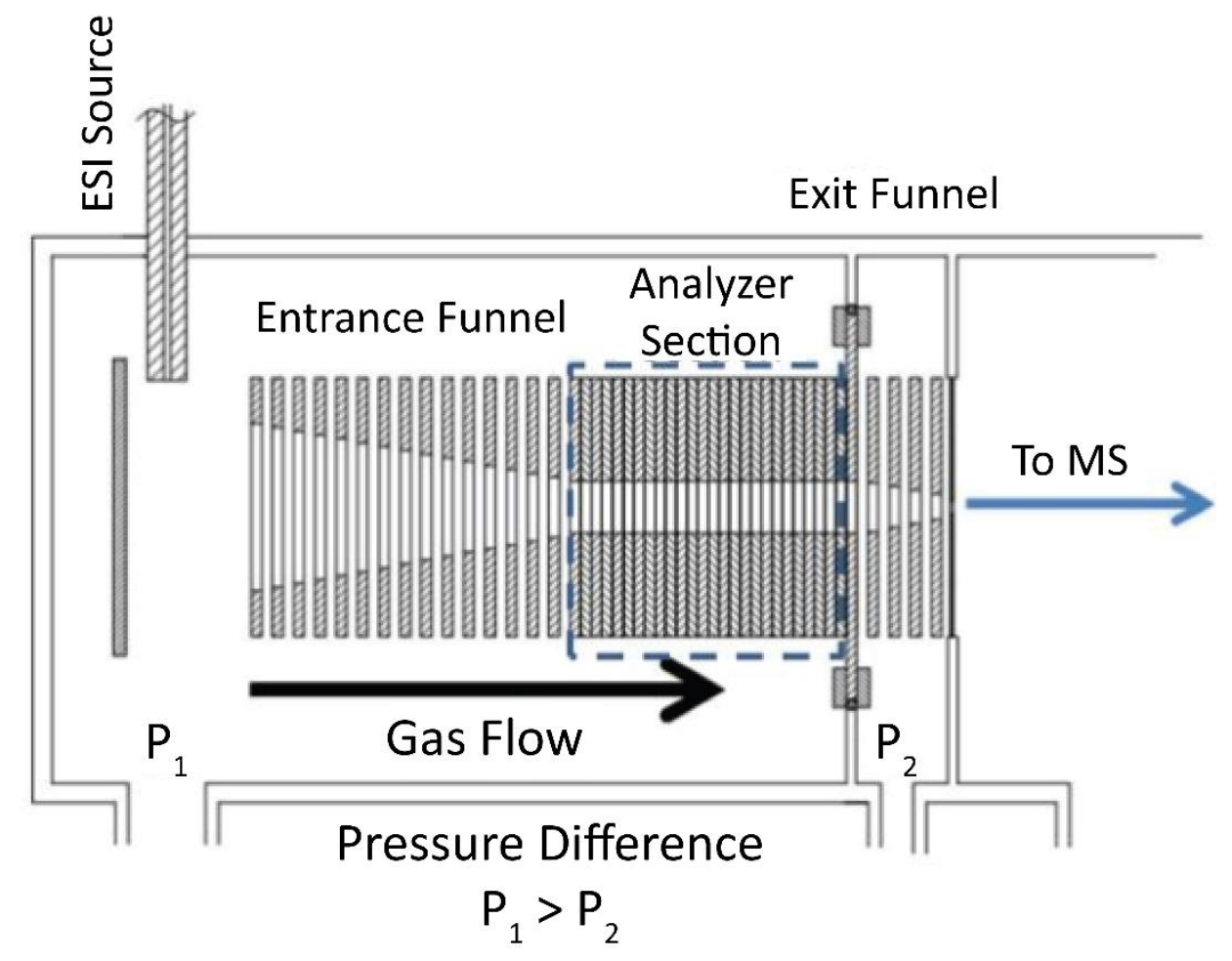


Figure S4.2. Experimental and theoretical mass shift for the deuterated and non-deuterated +6 and +13 charge states.
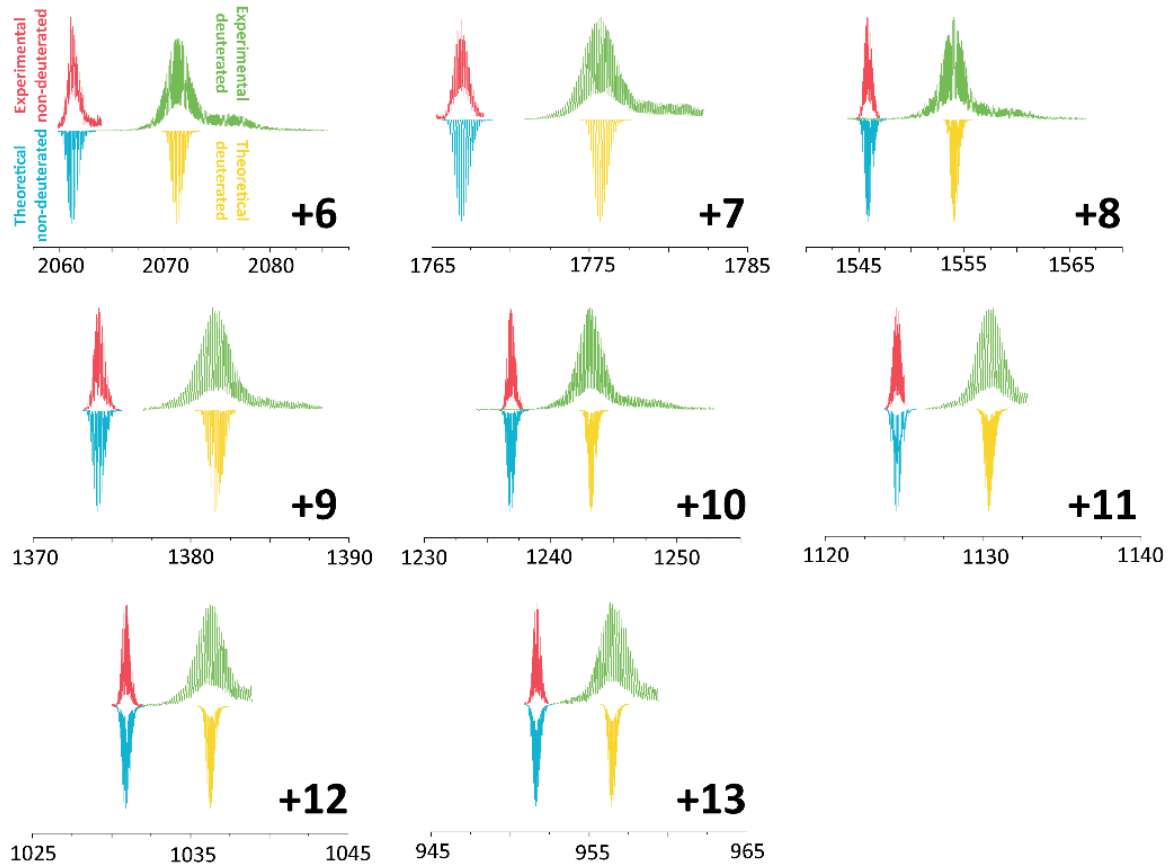
Figure S4.3. Evidence of $\mathrm{pH}$ induced denaturation of cyt $c$ by UV-VIS spectroscopy in the 300-600 nm spectral range. a) Samples with $10 \mu \mathrm{M}$ cyt $c$ at different ratios of solvent content, 5:95 of $\mathrm{MeOH}: \mathrm{H}_{2} \mathrm{O}$ at $\mathrm{pH} 4.6$ (blue line), 5:94:1 of $\mathrm{MeOH}: \mathrm{H}_{2} \mathrm{O}: \mathrm{AcOH}$ at $\mathrm{pH} 3.3$ (red line), 50:50 of $\mathrm{MeOH}: \mathrm{H}_{2} \mathrm{O}$ at $\mathrm{pH} 4.4$ (green line) and 50:49:1 of $\mathrm{MeOH}: \mathrm{H}_{2} \mathrm{O}: \mathrm{AcOH}$ at $\mathrm{pH} 3.0$ (yellow line). b) Samples at $5 \mu \mathrm{M}$ cyt $c$ at $\mathrm{pH} 3.3$ and ratios of solvent content of 5:94:1 (dark blue line), 10:89:1 (red line), 20:79:1 (green line), 30:69:1 (yellow line) and 40:59:1 (light blue line) of $\mathrm{MeOH}: \mathrm{H} 2 \mathrm{O}: \mathrm{AcOH}$. a) and b) show optical evidence of the denature of the protein under these conditions.

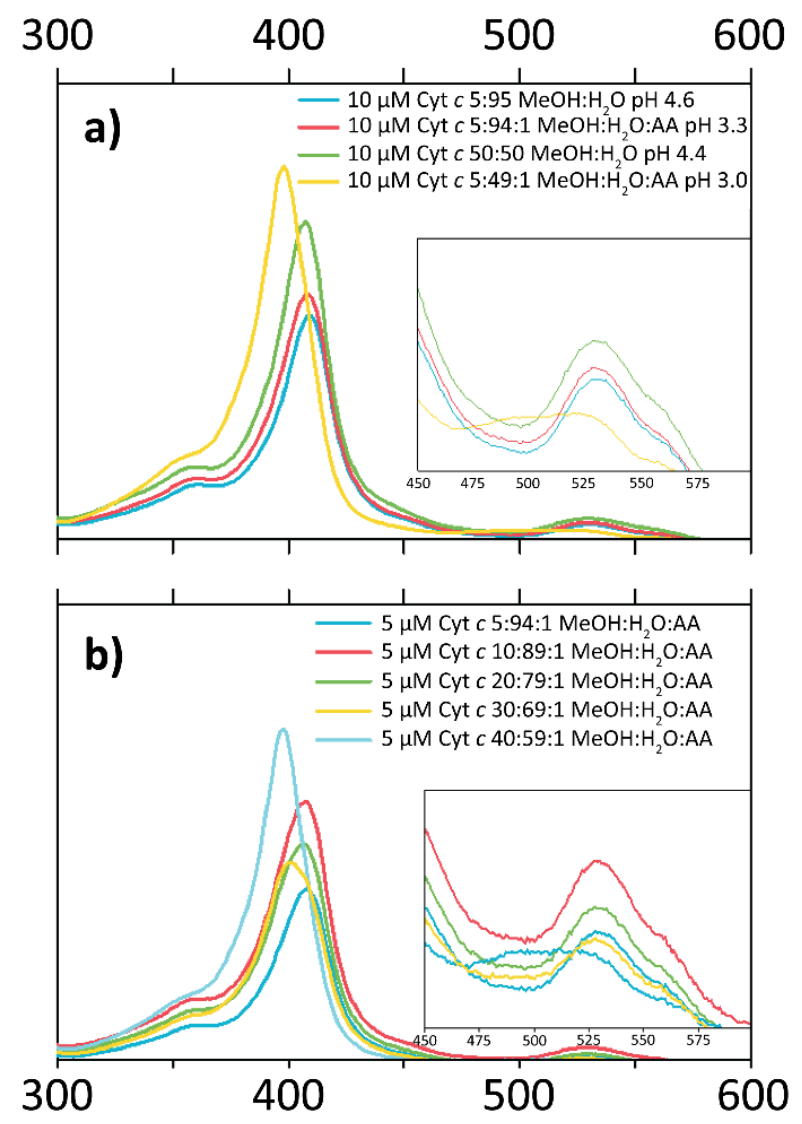


Figure S4.4. Summary of CCS literature values for cyt $c$.

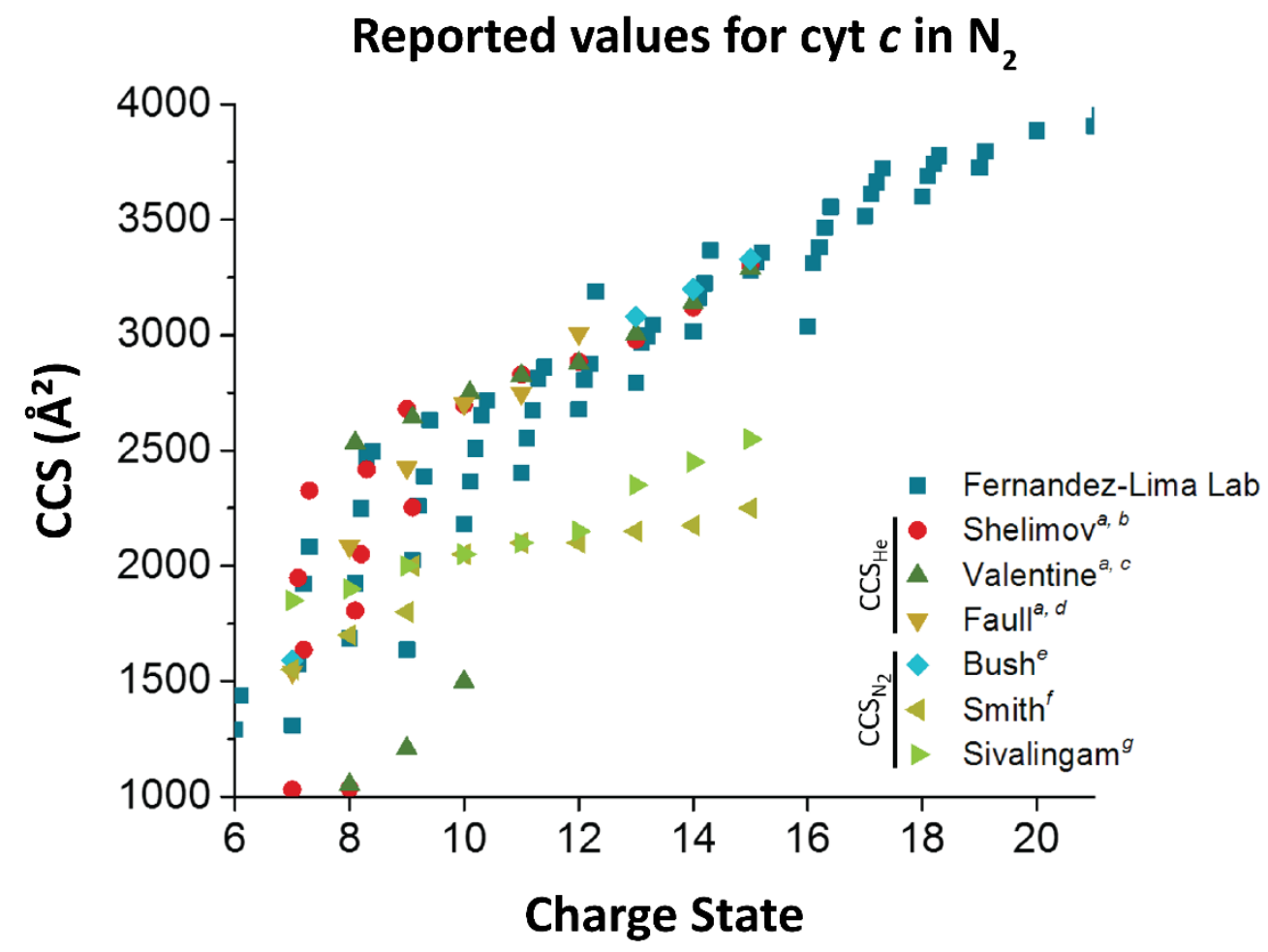

${ }^{a}$ The interpolation of the linear relationship resulting from plotting helium and nitrogen values reported by Bush et al., ${ }^{\mathrm{e}}$ was used to convert values originally measured in helium.

$$
\Omega_{H e}\left(\mathrm{~K}_{0}{ }^{\mathrm{N}_{2}}\right)=0.982 \times \Omega_{\mathrm{He}}\left(\mathrm{K}_{0}{ }^{\mathrm{He}}\right)+0.8
$$

${ }^{b}$ Shelimov, K. B.; Clemmer, D. E.; Hudgins, R. R.; Jarrold, M. F. J. Am. Chem. Soc. 1997, $119,2240-2248$.

${ }^{c}$ Valentine, S. J.; Clemmer, D. E. J. Am. Chem. Soc. 1997, 119 (15), 3558-3566.

${ }^{d}$ Faull, P. A.; Korkeila, K. E.; Kalapothakis, J. M.; Gray, A.; McCullough, B. J.; Barran, P. E. Int. J. Mass Spectrom. 2009, 283 (1-3), 140-148. 
${ }^{e}$ Bush, M. F.; Hall, Z.; Giles, K.; Hoyes, J.; Robinson, C. V; Ruotolo, B. T. Anal. Chem. 2010, 82 (22), 9557-9565.

${ }^{\text {f }}$ Smith, D.; Knapman, T.; Campuzano, I.; Malham, R.; Berryman, J.; Radford, S.; Ashcroft, A. Eur. J. Mass Spectrom. 2009, 15 (2), 113-130. 6

g Sivalingam, G. N.; Yan, J.; Sahota, H.; Thalassinos, K. Int. J. Mass Spectrom. 2013, 345$347,54-62$. 
Figure S4.5. HDX back exchange as a function of the trapping time and the incubation time, without CIA (blue panel) and with CIA (red panel).

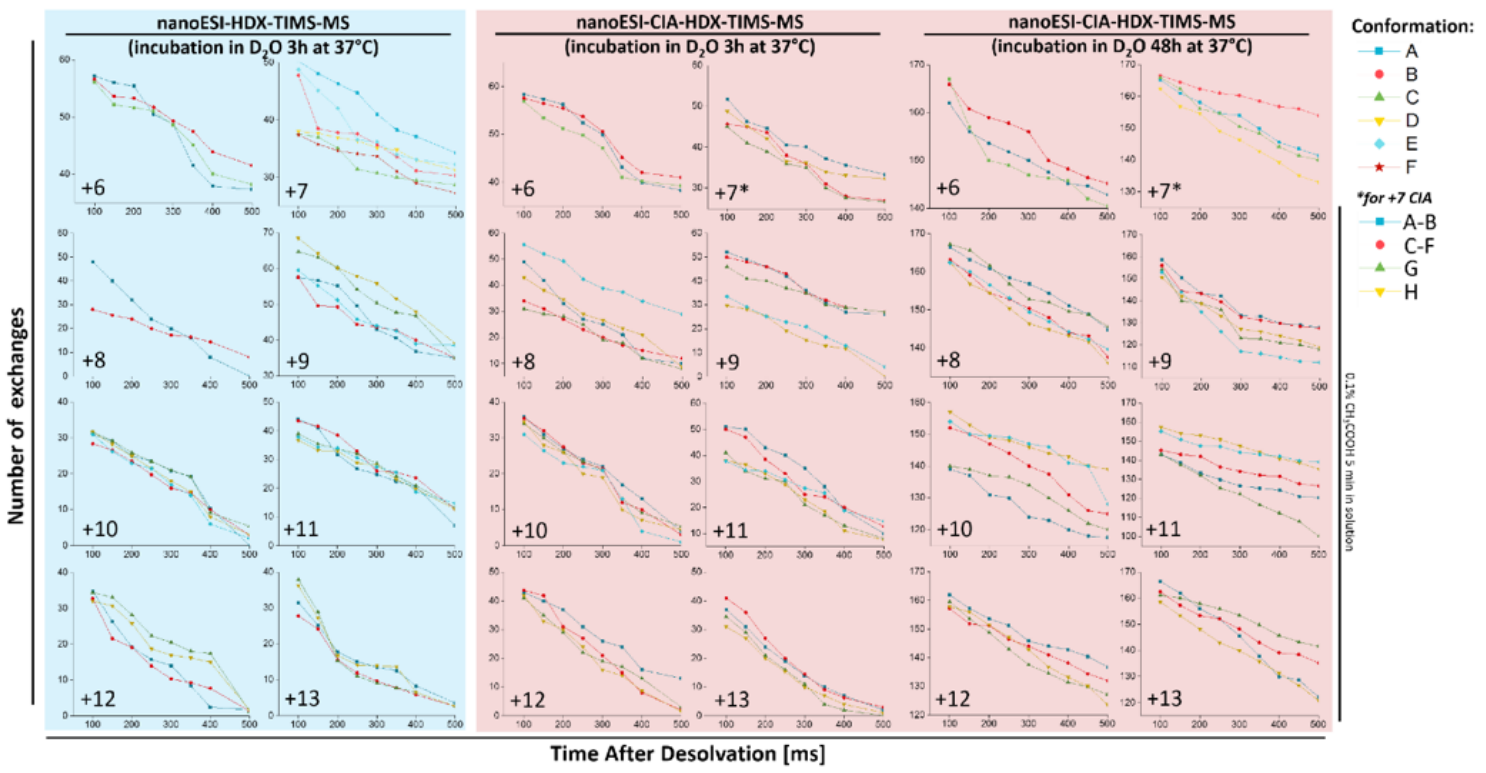


Figure S4.6. Clustering of HDX protection groups based on the number of deuterons and the number of conformations.
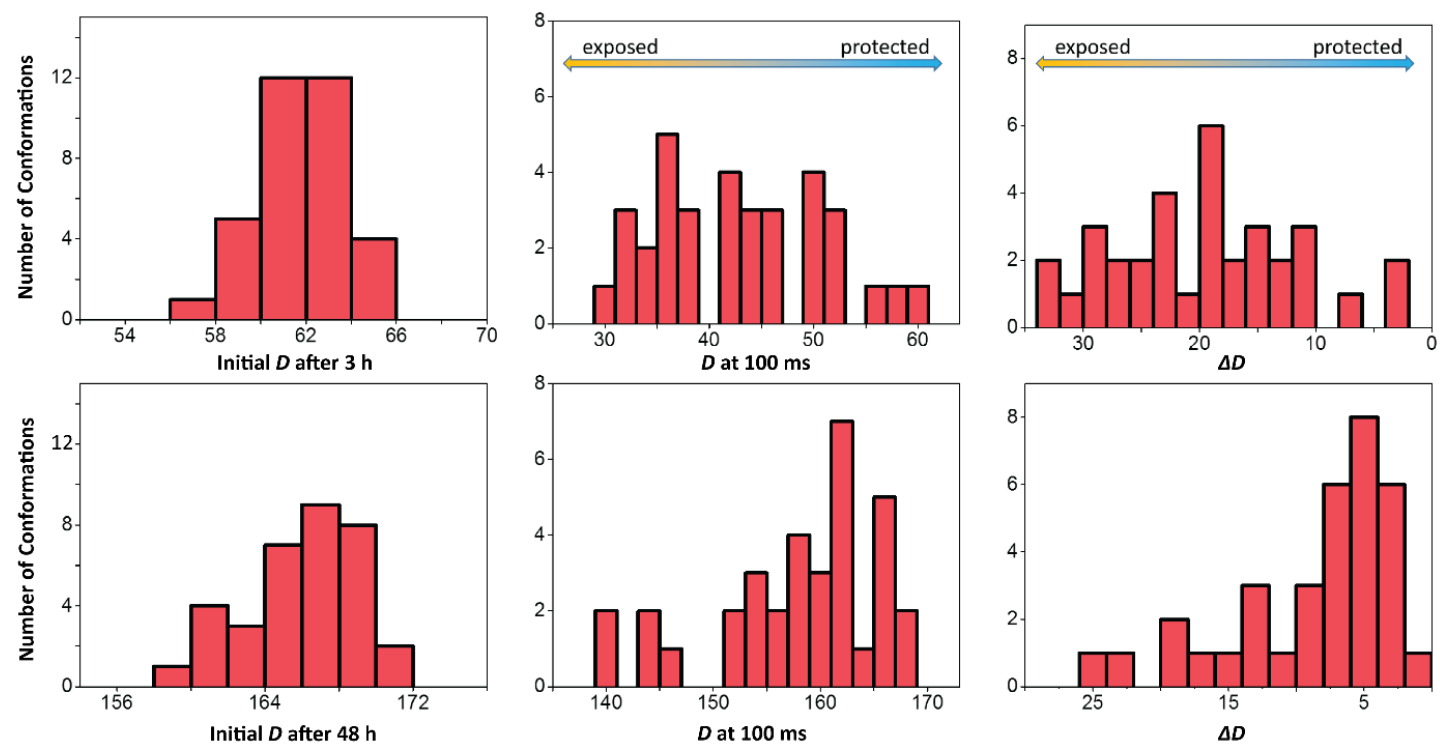
Figure S4.7. A. Comparison of the mass spectra of the +6 and -6 charge states of cyt $c$ after incubation in $\mathrm{D}_{2} \mathrm{O}$. B. Mass spectra of the +6 and -6 charge states of cyt $c$ as a function of the trapping time, showing the HDX back-exchange in both positive and negative mode. C. HDX back-exchange as a function of the trapping time for the +5 to +8 , and -5 and -6 charge states.

A.
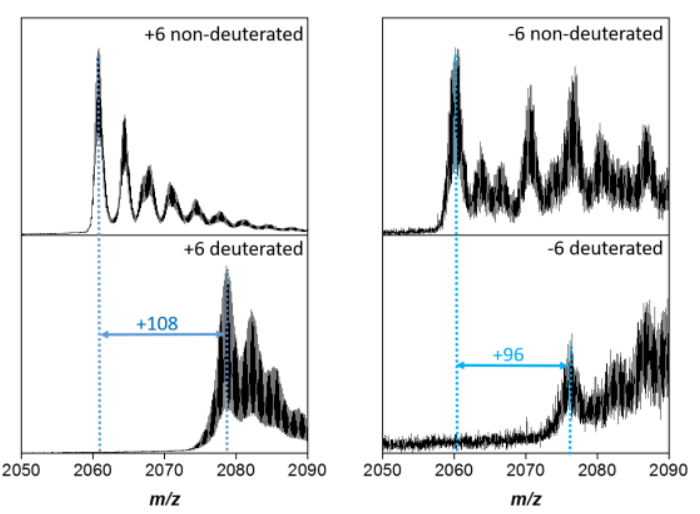

B.
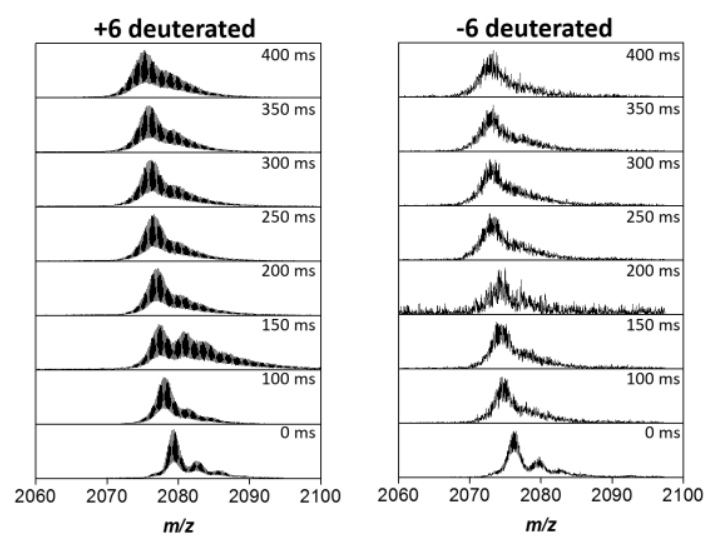

C.
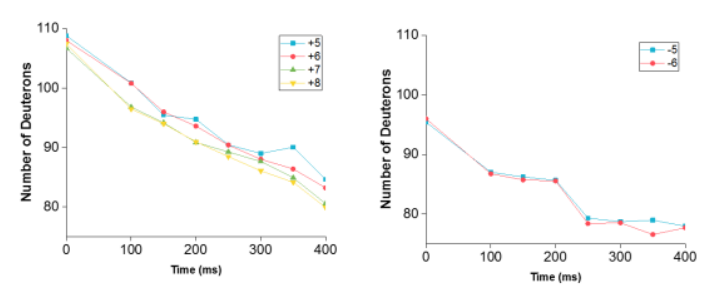
Figure S4.8. Unfolding pathway of cyt $c$.

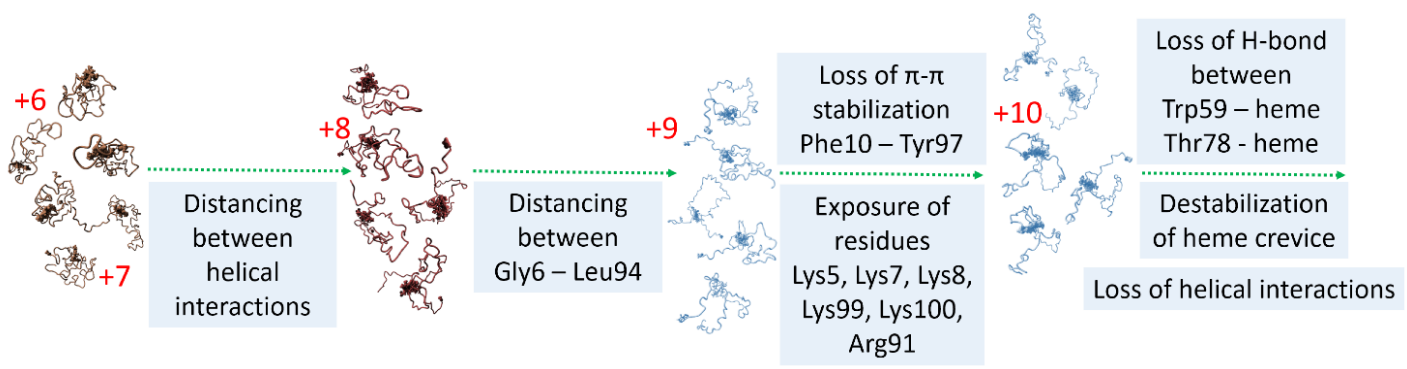


Table S4.1. Experimental results of HDX as a function of the incubation time and ionneutral collision cross section of cyt $c$ for +6 to +13 charge states.

\begin{tabular}{|c|c|c|c|c|c|c|c|c|c|c|}
\hline \multirow{2}{*}{\multicolumn{2}{|c|}{$[\mathrm{M}+\mathrm{nH}]^{+\mathrm{n}}$}} & \multicolumn{4}{|c|}{ Incubation Time in $\mathrm{D}_{2} \mathrm{O}$ of $3 \mathrm{~h}$} & \multicolumn{4}{|c|}{ Incubation Time in $\mathrm{D}_{2} \mathrm{O}$ of $48 \mathrm{~h}$} & \multirow{3}{*}{$\begin{array}{l}\mathrm{CCS}_{\mathrm{N}} \\
\left(\AA^{2}\right)\end{array}$} \\
\hline & & \multicolumn{3}{|c|}{ Number of deuterons } & \multirow{2}{*}{ Slope } & \multicolumn{3}{|c|}{ Number of deuterons } & \multirow{2}{*}{ Slope } & \\
\hline $\mathrm{z}$ & & at $0 \mathrm{~ms}$ & at $100 \mathrm{~ms}$ & at $500 \mathrm{~ms}$ & & at $0 \mathrm{~ms}$ & at $100 \mathrm{~ms}$ & at $500 \mathrm{~ms}$ & & \\
\hline & A & \multirow{3}{*}{$62 \pm 2$} & $59 \pm 4$ & $38 \pm 2$ & \begin{tabular}{|l}
$-0.06 \pm$ \\
0.01
\end{tabular} & \multirow{3}{*}{$171 \pm 3$} & $162 \pm 6$ & $143 \pm 4$ & \begin{tabular}{|l}
$-0.04 \pm$ \\
0.02
\end{tabular} & 1291 \\
\hline 6 & B & & $58 \pm 2$ & $41 \pm 2$ & \begin{tabular}{|l}
$-0.05 \pm$ \\
0.02
\end{tabular} & & $166 \pm 4$ & $145 \pm 3$ & \begin{tabular}{|l}
$-0.05 \pm$ \\
0.02
\end{tabular} & 1438 \\
\hline & C & & $56 \pm 7$ & $38 \pm 5$ & $\begin{array}{l}-0.04 \pm \\
0.01\end{array}$ & & $167 \pm 9$ & $140 \pm 7$ & \begin{tabular}{|l}
$-0.05 \pm$ \\
0.02
\end{tabular} & 1814 \\
\hline \multirow{8}{*}{7} & A & \multirow{8}{*}{$63 \pm 3$} & $50 \pm 1$ & $34 \pm 1$ & \begin{tabular}{|l}
$-0.07 \pm$ \\
0.03
\end{tabular} & \multirow{8}{*}{$169 \pm 4$} & $165 \pm 5$ & $141 \pm 3$ & \begin{tabular}{|l}
$-0.04 \pm$ \\
0.02 \\
\end{tabular} & 1279 \\
\hline & B & & $48 \pm 3$ & $30 \pm 3$ & $\begin{array}{l}-0.06 \pm \\
0.01\end{array}$ & & $165 \pm 5$ & $141 \pm 3$ & \begin{tabular}{|l|}
$-0.04 \pm$ \\
0.02
\end{tabular} & 1307 \\
\hline & $\mathrm{C}$ & & $38 \pm 3$ & $29 \pm 1$ & $\begin{array}{l}-0.02 \pm \\
0.01\end{array}$ & & $166 \pm 2$ & $140 \pm 3$ & $\begin{array}{l}-0.03 \pm \\
0.01\end{array}$ & 1574 \\
\hline & $\mathrm{D}$ & & $38 \pm 3$ & $31 \pm 4$ & $\begin{array}{l}-0.03 \pm \\
0.02\end{array}$ & & $166 \pm 2$ & $140 \pm 3$ & $\begin{array}{l}-0.04 \pm \\
0.01\end{array}$ & 1673 \\
\hline & E & & $49 \pm 2$ & $32 \pm 3$ & $\begin{array}{l}-0.07 \pm \\
0.02\end{array}$ & & $166 \pm 2$ & $140 \pm 3$ & $\begin{array}{l}-0.03 \pm \\
0.02\end{array}$ & 1722 \\
\hline & $\mathrm{F}$ & & $37 \pm 3$ & $27 \pm 3$ & $\begin{array}{l}-0.03 \pm \\
0.01\end{array}$ & & $166 \pm 2$ & $140 \pm 3$ & $\begin{array}{l}-0.04 \pm \\
0.02\end{array}$ & 1833 \\
\hline & G & & $45 \pm 1$ & $27 \pm 3$ & $\begin{array}{l}-0.04 \pm \\
0.02\end{array}$ & & $166 \pm 2$ & $140 \pm 3$ & $\begin{array}{l}-0.03 \pm \\
0.02\end{array}$ & 1927 \\
\hline & $\mathrm{H}$ & & $49 \pm 3$ & $32 \pm 1$ & $\begin{array}{l}-0.03 \pm \\
0.02\end{array}$ & & $162 \pm 3$ & $133 \pm 2$ & $\begin{array}{l}-0.04 \pm \\
0.02\end{array}$ & 2178 \\
\hline \multirow{5}{*}{8} & A & \multirow{5}{*}{$64 \pm 4$} & $49 \pm 6$ & $10 \pm 5$ & \begin{tabular}{|l}
$-0.10 \pm$ \\
0.03
\end{tabular} & \multirow{5}{*}{$169 \pm 4$} & $166 \pm 2$ & $145 \pm 3$ & \begin{tabular}{|l|}
$-0.05 \pm$ \\
0.01
\end{tabular} & 1682 \\
\hline & B & & $34 \pm 4$ & $12 \pm 3$ & \begin{tabular}{|l|}
$-0.06 \pm$ \\
0.02
\end{tabular} & & $163 \pm 5$ & $138 \pm 1$ & $\begin{array}{l}-0.06 \pm \\
0.01\end{array}$ & 1925 \\
\hline & C & & $31 \pm 3$ & $8 \pm 2$ & \begin{tabular}{|l}
$-0.06 \pm$ \\
0.01
\end{tabular} & & $167 \pm 3$ & $146 \pm 1$ & $\begin{array}{l}-0.06 \pm \\
0.02\end{array}$ & 2249 \\
\hline & D & & $43 \pm 4$ & $8 \pm 2$ & \begin{tabular}{|l|}
$-0.08 \pm$ \\
0.03
\end{tabular} & & $162 \pm 2$ & $136 \pm 4$ & $\begin{array}{l}-0.06 \pm \\
0.02\end{array}$ & 2468 \\
\hline & E & & $56 \pm 5$ & $29 \pm 3$ & $\begin{array}{l}-0.07 \pm \\
0.02\end{array}$ & & $162 \pm 6$ & $140 \pm 2$ & $\begin{array}{l}-0.06 \pm \\
0.01\end{array}$ & 2496 \\
\hline \multirow{2}{*}{9} & A & \multirow{2}{*}{$65 \pm 3$} & $52 \pm 6$ & $26 \pm 1$ & $\begin{array}{l}-0.07 \pm \\
0.02\end{array}$ & \multirow{2}{*}{$167 \pm 4$} & $159 \pm 3$ & $128 \pm 2$ & \begin{tabular}{|l|}
$-0.08 \pm$ \\
0.01
\end{tabular} & 1637 \\
\hline & B & & $50 \pm 4$ & $27 \pm 2$ & \begin{tabular}{|l}
$-0.07 \pm$ \\
0.01
\end{tabular} & & $156 \pm 4$ & $127 \pm 4$ & $\begin{array}{l}-0.07 \pm \\
0.02\end{array}$ & 2024 \\
\hline
\end{tabular}




\begin{tabular}{|c|c|c|c|c|c|c|c|c|c|c|}
\hline & $\mathrm{C}$ & & $46 \pm 7$ & $27 \pm 2$ & $\begin{array}{l}-0.05 \pm \\
0.03\end{array}$ & & $154 \pm 4$ & $118 \pm 3$ & \begin{tabular}{|l}
$-0.09 \pm$ \\
0.02
\end{tabular} & 2258 \\
\hline & D & & $30 \pm 3$ & $0 \pm 1$ & \begin{tabular}{|l}
$-0.07 \pm$ \\
0.03
\end{tabular} & & $151 \pm 2$ & $119 \pm 3$ & \begin{tabular}{|l}
$-0.08 \pm$ \\
0.01
\end{tabular} & 2631 \\
\hline & $\mathrm{E}$ & & $35 \pm 4$ & $4 \pm 1$ & $\begin{array}{l}-0.07 \pm \\
0.02\end{array}$ & & $153 \pm 3$ & $112 \pm 4$ & $\begin{array}{l}-0.10 \pm \\
0.03\end{array}$ & 2388 \\
\hline & A & & $36 \pm 4$ & $4 \pm 2$ & $\begin{array}{l}-0.08 \pm \\
0.01\end{array}$ & & $139 \pm 5$ & $118 \pm 3$ & $\begin{array}{l}-0.06 \pm \\
0.02\end{array}$ & 2181 \\
\hline & B & & $36 \pm 4$ & $3 \pm 1$ & \begin{tabular}{|l}
$-0.08 \pm$ \\
0.02
\end{tabular} & & $152 \pm 3$ & $125 \pm 4$ & \begin{tabular}{|l}
$-0.07 \pm$ \\
0.02
\end{tabular} & 2365 \\
\hline 10 & $\mathrm{C}$ & $62 \pm 5$ & $34 \pm 3$ & $5 \pm 2$ & $\begin{array}{l}-0.08 \pm \\
0.02\end{array}$ & $164 \pm 5$ & $140 \pm 4$ & $120 \pm 5$ & $\begin{array}{l}-0.05 \pm \\
0.01\end{array}$ & 2508 \\
\hline & D & & $35 \pm 5$ & $4 \pm 2$ & $\begin{array}{l}-0.08 \pm \\
0.03\end{array}$ & & $157 \pm 3$ & $139 \pm 4$ & $\begin{array}{l}-0.04 \pm \\
0.02\end{array}$ & 2652 \\
\hline & $\mathrm{E}$ & & $31 \pm 2$ & $1 \pm 1$ & $\begin{array}{l}-0.08 \pm \\
0.01\end{array}$ & & $154 \pm 4$ & $128 \pm 3$ & $\begin{array}{l}-0.05 \pm \\
0.02\end{array}$ & 2717 \\
\hline & A & & $51 \pm 3$ & $10 \pm 2$ & $\begin{array}{l}-1.09 \pm \\
0.09\end{array}$ & & $143 \pm 2$ & $120 \pm 4$ & \begin{tabular}{|l}
$-0.06 \pm$ \\
0.02
\end{tabular} & 2403 \\
\hline & B & & $50 \pm 2$ & $13 \pm 3$ & $\begin{array}{l}-0.10 \pm \\
0.01\end{array}$ & & $145 \pm 3$ & $127 \pm 2$ & $\begin{array}{l}-0.05 \pm \\
0.02\end{array}$ & 2553 \\
\hline 11 & $\mathrm{C}$ & $61 \pm 5$ & $41 \pm 3$ & $8 \pm 2$ & \begin{tabular}{|l}
$-0.08 \pm$ \\
0.02
\end{tabular} & $161 \pm 5$ & $143 \pm 2$ & $100 \pm 4$ & \begin{tabular}{|l}
$-0.10 \pm$ \\
0.02
\end{tabular} & 2676 \\
\hline & D & & $38 \pm 3$ & $7 \pm 2$ & $\begin{array}{l}-0.09 \pm \\
0.03\end{array}$ & & $157 \pm 2$ & $135 \pm 3$ & $\begin{array}{l}-0.06 \pm \\
0.01\end{array}$ & 2813 \\
\hline & $\mathrm{E}$ & & $38 \pm 5$ & $15 \pm 1$ & \begin{tabular}{|l}
$-0.06 \pm$ \\
0.01
\end{tabular} & & $155 \pm 7$ & $139 \pm 3$ & \begin{tabular}{|l}
$-0.37 \pm$ \\
0.07
\end{tabular} & 2861 \\
\hline & A & & $43 \pm 4$ & $13 \pm 2$ & $\begin{array}{l}-0.09 \pm \\
0.03\end{array}$ & & $162 \pm 2$ & $137 \pm 3$ & $\begin{array}{l}-0.06 \pm \\
0.01\end{array}$ & 2678 \\
\hline 12 & B & $61+5$ & $44 \pm 4$ & $2 \pm 1$ & $\begin{array}{l}-1.11 \pm \\
0.06\end{array}$ & $166+5$ & $157 \pm 6$ & $132 \pm 3$ & \begin{tabular}{|l}
$-0.06 \pm$ \\
0.01
\end{tabular} & 2806 \\
\hline 12 & $\mathrm{C}$ & $61 \pm 5$ & $41 \pm 3$ & $3 \pm 1$ & $\begin{array}{l}-0.09 \pm \\
0.02\end{array}$ & $166 \pm 5$ & $160 \pm 3$ & $127 \pm 2$ & \begin{tabular}{|l}
$-0.08 \pm$ \\
0.01
\end{tabular} & 2876 \\
\hline & D & & $42 \pm 5$ & $1 \pm 1$ & $\begin{array}{l}-0.10 \pm \\
0.03\end{array}$ & & $158 \pm 3$ & $123 \pm 4$ & $\begin{array}{l}-0.09 \pm \\
0.02\end{array}$ & 3187 \\
\hline & A & & $37 \pm 4$ & $2 \pm 1$ & $\begin{array}{l}-0.09 \pm \\
0.01\end{array}$ & & $166 \pm 6$ & $122 \pm 3$ & $\begin{array}{l}-0.11 \pm \\
0.04\end{array}$ & 2795 \\
\hline 13 & B & $59+5$ & $41 \pm 2$ & $3 \pm 2$ & $\begin{array}{l}-0.10 \pm \\
0.03\end{array}$ & $168+5$ & $162 \pm 3$ & $135 \pm 4$ & $\begin{array}{l}-0.07 \pm \\
0.01\end{array}$ & 2969 \\
\hline 13 & $\mathrm{C}$ & p9. & $35 \pm 4$ & $0 \pm 1$ & $\begin{array}{l}-0.09 \pm \\
0.03\end{array}$ & $108 \pm 5$ & $161 \pm 2$ & $142 \pm 3$ & $\begin{array}{l}-0.05 \pm \\
0.01\end{array}$ & 2998 \\
\hline & D & & $31 \pm 3$ & $1 \pm 2$ & $\begin{array}{l}-0.08 \pm \\
0.01\end{array}$ & & $159 \pm 5$ & $215 \pm 2$ & $\begin{array}{l}-0.09 \pm \\
0.03\end{array}$ & 3044 \\
\hline
\end{tabular}


Table S4.2. Experimental ion-neutral collision cross section of cyt $c$.

\begin{tabular}{|c|c|c|c|}
\hline \multirow{2}{*}{$\frac{[\mathrm{M}+\mathrm{nH}]^{+\mathrm{n}}}{z}$} & & \multicolumn{2}{|c|}{ TIMS Experimental } \\
\hline & & $\mathrm{Ko}\left(\mathrm{cm}^{2} / \mathrm{Vs}\right)$ & $\operatorname{CCS}\left[\AA^{2}\right]$ \\
\hline \multirow{4}{*}{14} & A & 0.932 & 3017 \\
\hline & B & 0.889 & 3162 \\
\hline & $\mathrm{C}$ & 0.872 & 3223 \\
\hline & $\mathrm{D}$ & 0.835 & 3367 \\
\hline \multirow{3}{*}{15} & A & 0.918 & 3279 \\
\hline & B & 0.908 & 3318 \\
\hline & $\mathrm{C}$ & 0.897 & 3358 \\
\hline \multirow{5}{*}{16} & A & 1.058 & 3036 \\
\hline & B & 0.970 & 3311 \\
\hline & $\mathrm{C}$ & 0.950 & 3382 \\
\hline & $\mathrm{D}$ & 0.927 & 3465 \\
\hline & $\mathrm{E}$ & 0.903 & 3557 \\
\hline \multirow{4}{*}{17} & A & 0.971 & 3516 \\
\hline & B & 0.945 & 3611 \\
\hline & $\mathrm{C}$ & 0.932 & 3663 \\
\hline & $\mathrm{D}$ & 0.916 & 3724 \\
\hline \multirow{4}{*}{18} & A & 1.004 & 3598 \\
\hline & $\mathrm{B}$ & 0.980 & 3689 \\
\hline & $\mathrm{C}$ & 0.965 & 3743 \\
\hline & $\mathrm{D}$ & 0.957 & 3778 \\
\hline \multirow[t]{2}{*}{19} & $\mathrm{~A}$ & 1.023 & 3727 \\
\hline & $\mathrm{B}$ & 1.004 & 3798 \\
\hline 20 & A & 1.034 & 3885 \\
\hline \multirow{2}{*}{21} & $\mathrm{~A}$ & 1.079 & 3907 \\
\hline & B & 1.066 & 3954 \\
\hline
\end{tabular}


Table S4.3. Experimental ion-neutral collision cross section of cyt $c$ for -5 to -10 charge states.

\begin{tabular}{|c|c|c|}
\hline$[\mathrm{M}-\mathrm{nH}]^{-\mathrm{n}}$ & & $\begin{array}{l}C_{C} S_{\mathrm{N} 2} \\
\left(\AA^{2}\right)\end{array}$ \\
\hline \multirow{3}{*}{$5 ; 6$} & A & 1291 \\
\hline & B & 1438 \\
\hline & C & 1814 \\
\hline \multirow{4}{*}{7} & A & 1929 \\
\hline & B & 2258 \\
\hline & $\mathrm{C}$ & 2473 \\
\hline & $\mathrm{D}$ & 2942 \\
\hline \multirow{6}{*}{8} & A & 1924 \\
\hline & B & 2041 \\
\hline & $\mathrm{C}$ & 2259 \\
\hline & $\mathrm{D}$ & 2376 \\
\hline & $\mathrm{E}$ & 2492 \\
\hline & $\mathrm{F}$ & 2921 \\
\hline \multirow{2}{*}{9} & A & 2175 \\
\hline & B & 2409 \\
\hline \multirow{2}{*}{10} & A & 2337 \\
\hline & $B$ & 2514 \\
\hline
\end{tabular}


SUPPORTING INFORMATION FOR CHAPTER 5. STRUCTURAL

CHARACTERIZATION OF THE KNOT PROTEIN YIBK USING TRAPPED ION MOBILITY SPECTROMETRY - MASS SPECTROMETRY AND ENZYMATIC

DIGESTION

Figure S5.1. TIMS instrument scheme

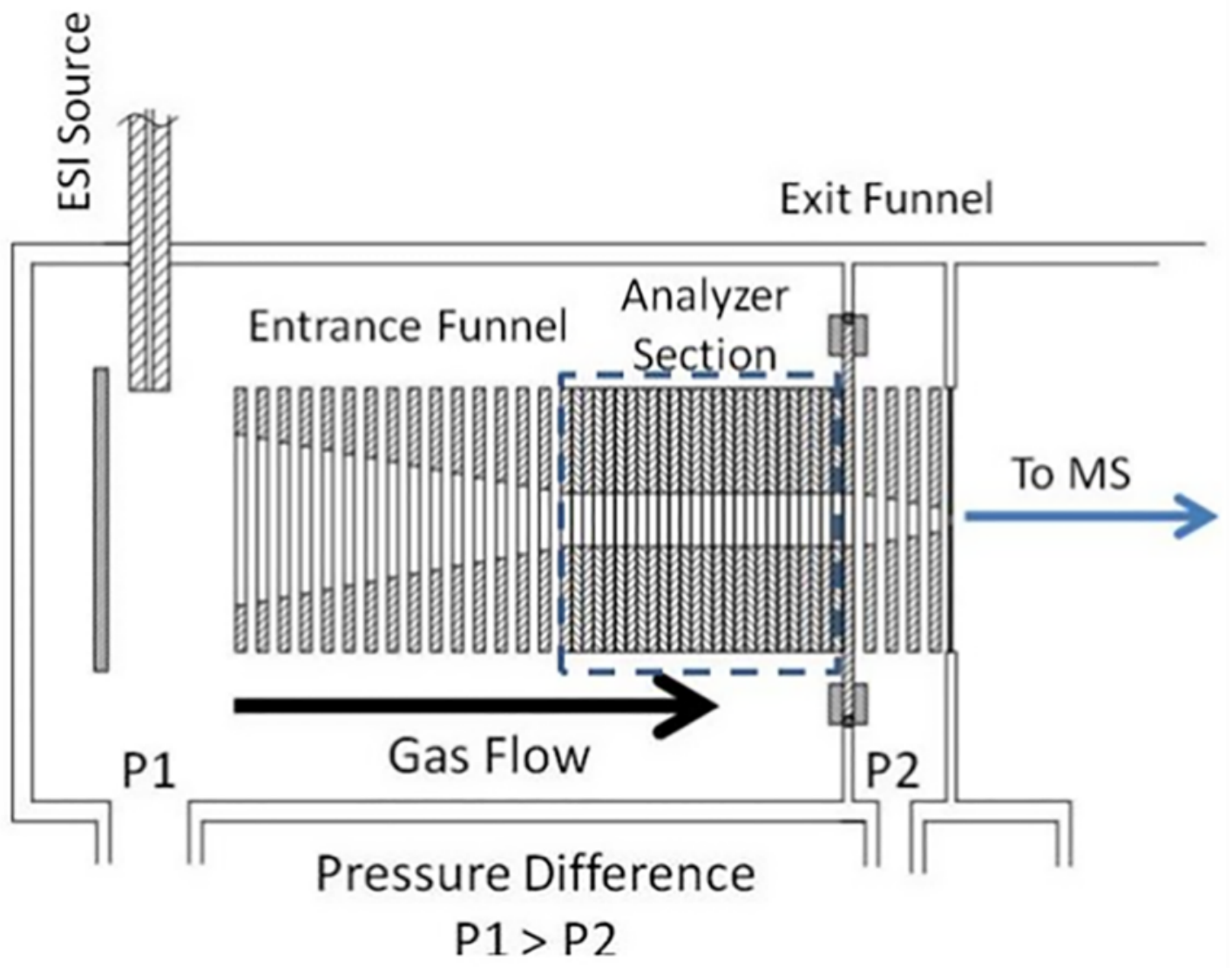


Figure S5.2. Contour plots of the +7 to +22 charge states of YibK as a function of the organic content (e.g., \% $\mathrm{MeOH}$ )
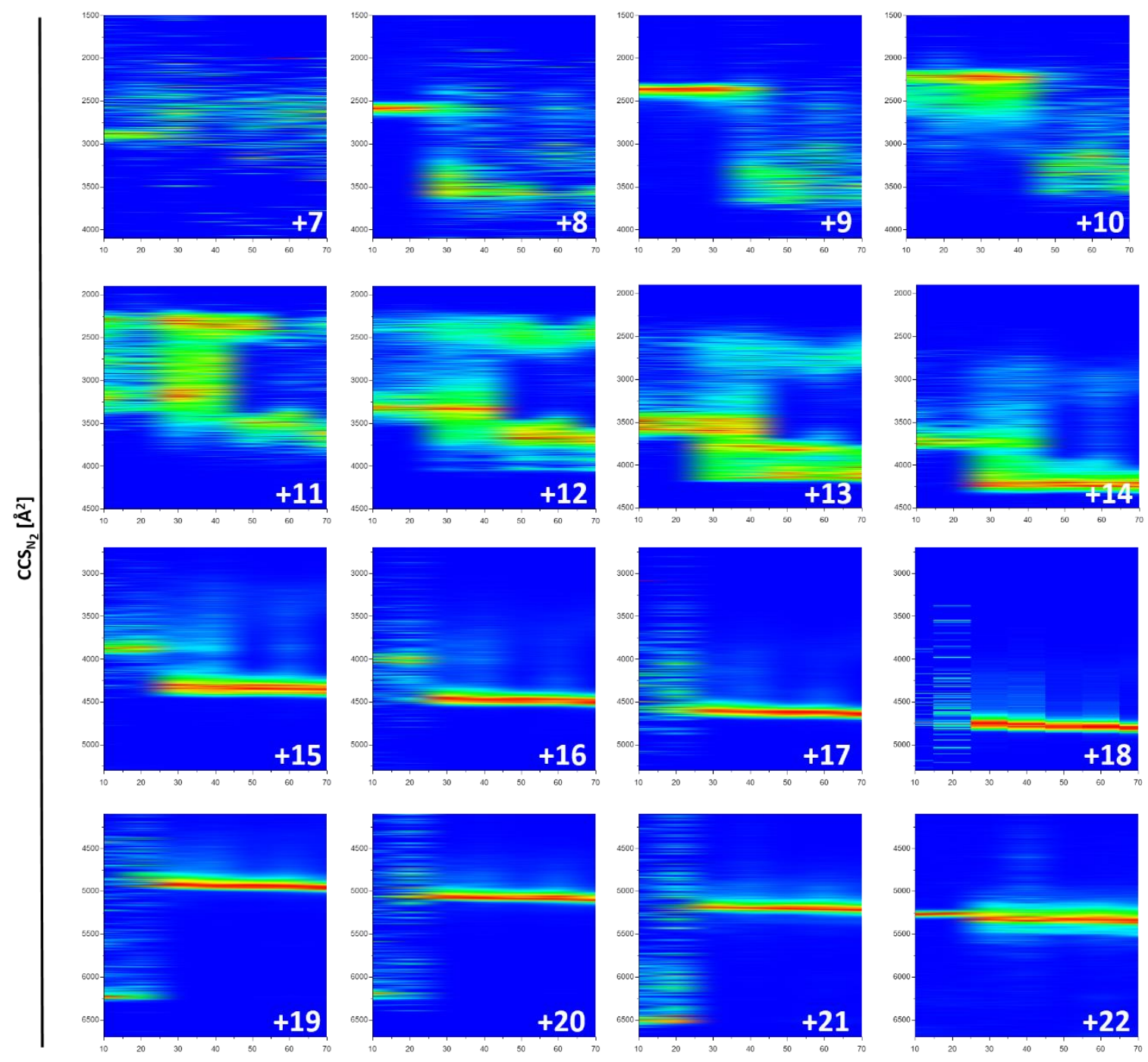

Organic Content [\% MeOH] 
Figure S5.3. Contour plots of the +7 to +22 charge states of YibK as a function of the activation energy (e.g., deflector voltage)

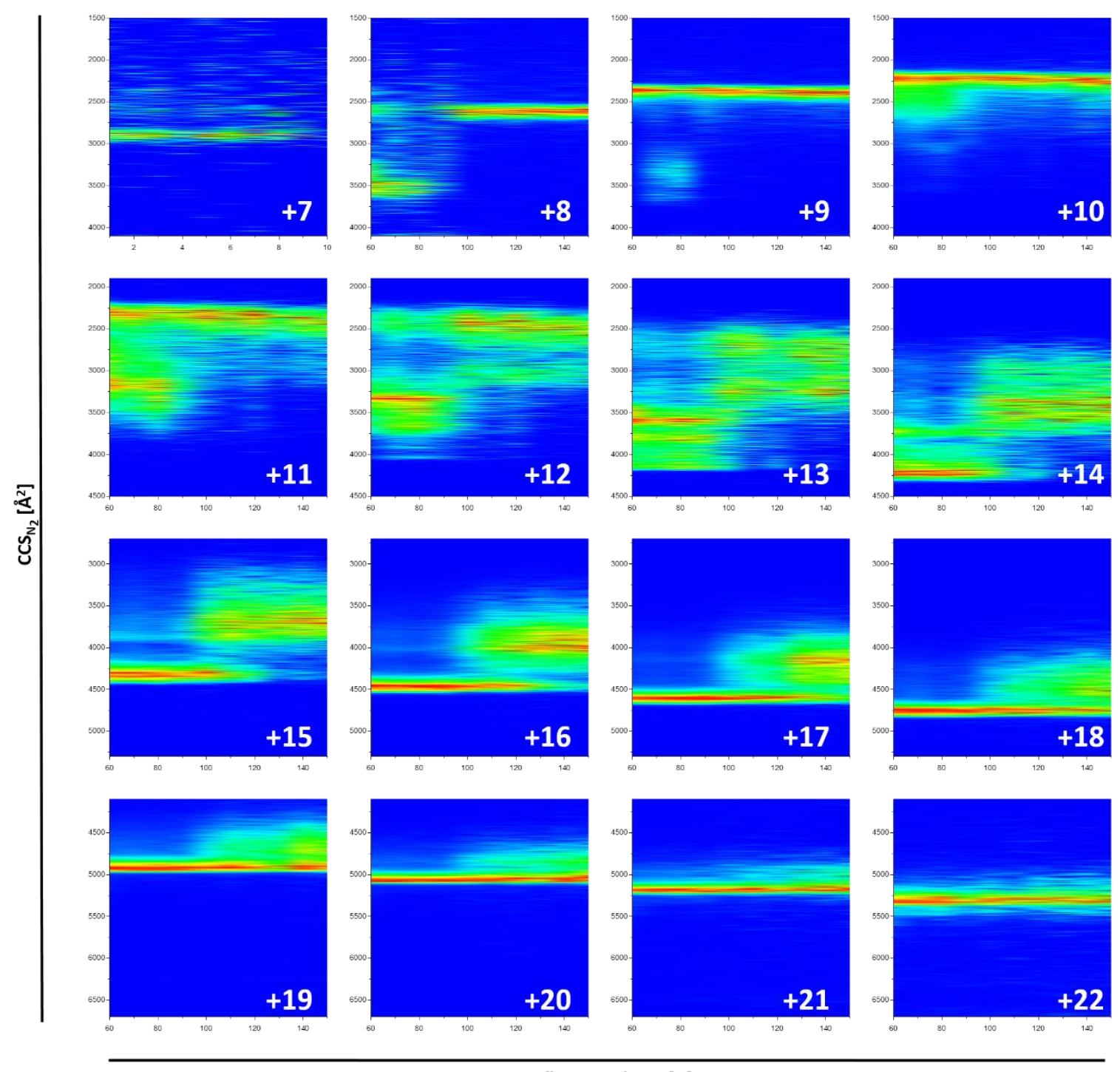

Deflector Voltage [V] 
Table S5.1. Experimental and theoretical $\mathrm{CCS}_{\mathrm{N} 2}$ of the observed fragments after digestion with CPDY.

\begin{tabular}{|c|c|c|c|c|}
\hline Fragment & Mass (Da) & $\begin{array}{l}\text { Charge } \\
\text { State }\end{array}$ & $\begin{array}{c}\text { Experimental } \mathrm{CCS}_{\mathrm{N} 2}\left(\mathrm{~A}^{2}\right) \\
\text { Most Intense Band }\end{array}$ & $\begin{array}{l}\text { Theoretical CCS } \mathrm{N}^{2} \\
\qquad\left(\mathrm{~A}^{2}\right) \\
\text { Model preserves } \\
\text { integrity of the core }\end{array}$ \\
\hline $1-173$ & 20059 & $\begin{array}{r}+7 \\
+8 \\
+11 \\
+12 \\
+13 \\
+15\end{array}$ & $\begin{array}{l}1932 \\
1958 \\
2546 \\
3426 \\
3565 \\
3856\end{array}$ & $\begin{array}{l}2481 \\
2502 \\
2584 \\
2616 \\
2647 \\
2733\end{array}$ \\
\hline $1-161$ & 18789 & $\begin{array}{r}+14 \\
+17\end{array}$ & $\begin{array}{l}3996 \\
4107\end{array}$ & $\begin{array}{l}2634 \\
2753\end{array}$ \\
\hline $1-156$ & 18083 & $\begin{array}{l}+16 \\
+17\end{array}$ & $\begin{array}{l}4193 \\
3678\end{array}$ & $\begin{array}{l}2658 \\
2682\end{array}$ \\
\hline $1-145$ & 16967 & $\begin{array}{l}+7 \\
+19\end{array}$ & $\begin{array}{l}1746 \\
5225\end{array}$ & $\begin{array}{l}2238 \\
2552\end{array}$ \\
\hline $1-141$ & 16522 & $\begin{array}{l}+12 \\
+14\end{array}$ & $\begin{array}{l}2672 \\
3690\end{array}$ & $\begin{array}{l}2281 \\
2336\end{array}$ \\
\hline $1-120$ & 14096 & +6 & 1549 & 2067 \\
\hline
\end{tabular}




\begin{tabular}{|c|c|c|c|c|}
\hline & & $\begin{array}{l}+7 \\
+8 \\
+9 \\
+10\end{array}$ & $\begin{array}{l}1595 \\
1958 \\
2282 \\
2255\end{array}$ & $\begin{array}{l}2083 \\
2102 \\
2123 \\
2142\end{array}$ \\
\hline $1-108$ & 12772 & $\begin{array}{r}+9 \\
+17\end{array}$ & $\begin{array}{l}2050 \\
3102\end{array}$ & $\begin{array}{l}2024 \\
2229\end{array}$ \\
\hline $1-101$ & 11941 & $\begin{array}{l}+14 \\
+16\end{array}$ & $\begin{array}{l}2686 \\
2792\end{array}$ & $\begin{array}{l}2120 \\
2194\end{array}$ \\
\hline $1-155$ & 17984 & +15 & 3717 & 2579 \\
\hline $1-151$ & 17614 & +14 & 4049 & 2499 \\
\hline $1-149$ & 17412 & +14 & 3532 & 2442 \\
\hline $1-147$ & 17212 & +15 & 3796 & 2444 \\
\hline $1-143$ & 16724 & +13 & 2838 & 2316 \\
\hline $1-140$ & 16451 & +16 & 3580 & 2400 \\
\hline $1-138$ & 16219 & +14 & 4789 & 2326 \\
\hline $1-134$ & 15739 & +12 & 2401 & 2264 \\
\hline $1-130$ & 15223 & +12 & 2606 & 2239 \\
\hline $1-128$ & 14994 & +14 & 2996 & 2288 \\
\hline
\end{tabular}




\begin{tabular}{|c|c|c|c|c|}
\hline $1-121$ & 14210 & +13 & 2348 & 2228 \\
\hline $1-119$ & 14039 & +11 & 2496 & 2169 \\
\hline $1-115$ & 13556 & +10 & 3035 & 2100 \\
\hline \multirow{2}{*}{$26-175$} & \multirow{2}{*}{17197} & +13 & 2825 & 2485 \\
\hline & & +14 & 3465 & 2516 \\
\hline \multirow{2}{*}{$33-175$} & \multirow{2}{*}{16473} & +17 & 3671 & 2573 \\
\hline & & +18 & 5106 & 2612 \\
\hline \multirow{2}{*}{$40-175$} & \multirow{2}{*}{15689} & +11 & 2481 & 2316 \\
\hline & & +13 & 3136 & 2361 \\
\hline \multirow{2}{*}{$54-175$} & \multirow{2}{*}{14106} & +12 & 3162 & 2188 \\
\hline & & +14 & 4107 & 2232 \\
\hline \multirow{3}{*}{$77-175$} & \multirow{3}{*}{11167} & +9 & 1865 & 1949 \\
\hline & & +10 & 2394 & 1983 \\
\hline & & +15 & 2725 & 2183 \\
\hline \multirow{2}{*}{$90-137$} & \multirow{2}{*}{5439} & +4 & 900 & 1014 \\
\hline & & +5 & 1144 & 1026 \\
\hline \multirow{2}{*}{$89-137$} & \multirow{2}{*}{5595} & +5 & 1261 & 1007 \\
\hline & & +6 & 1430 & 1038 \\
\hline $91-135$ & 5057 & +4 & 1070 & 990 \\
\hline
\end{tabular}




\begin{tabular}{|c|c|c|c|c|}
\hline $90-135$ & 5170 & +4 & 944 & 970 \\
\hline $92-138$ & 5276 & +5 & 1283 & 1092 \\
\hline $85-141$ & 6478 & +6 & 1541 & 1023 \\
\hline
\end{tabular}


SUPPORTING INFORMATION FOR CHAPTER 6. INSIGHTS FROM TRAPPED

ION MOBILITY SPECTROMETRY - MASS SPECTROMETRY ON THE

STRUCTURAL INTEGRITY OF THE KNOT PROTEIN UBIQUITIN C-TERMINAL

HYDROLASE

Figure S6.1. TIMS instrument scheme

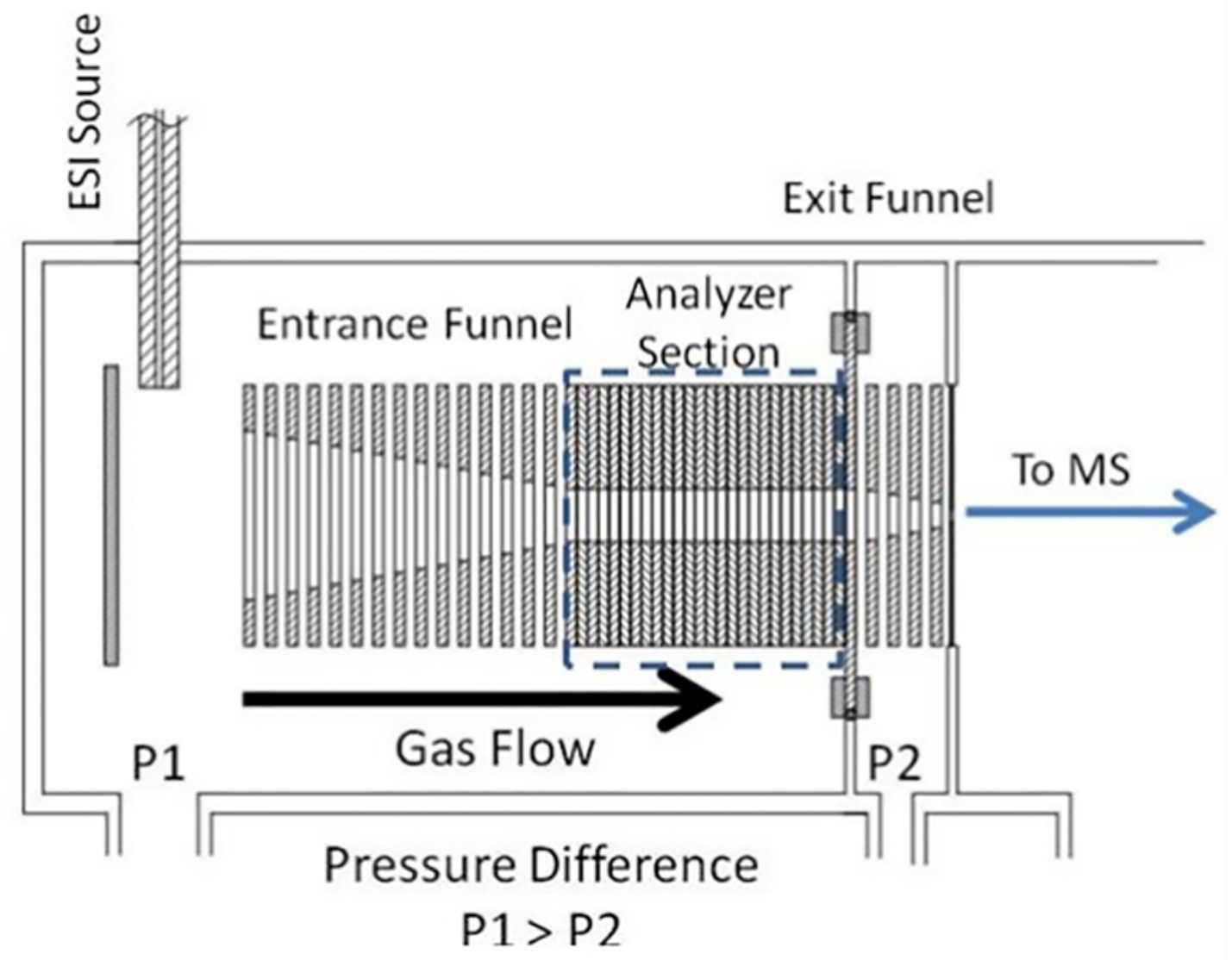


Figure S6.2. Contour plots of the charge states of UCH as a function of the organic content (e.g., \% MeOH). The solution conditions are labeled as follows: 1: $0 \% \mathrm{MeOH} ; 2: 5 \%$ $\mathrm{MeOH}$; 3: 10\% MeOH; 4: 20\% MeOH; 5: 30\% MeOH; 6: 40\% MeOH; 7: 50\% MeOH.

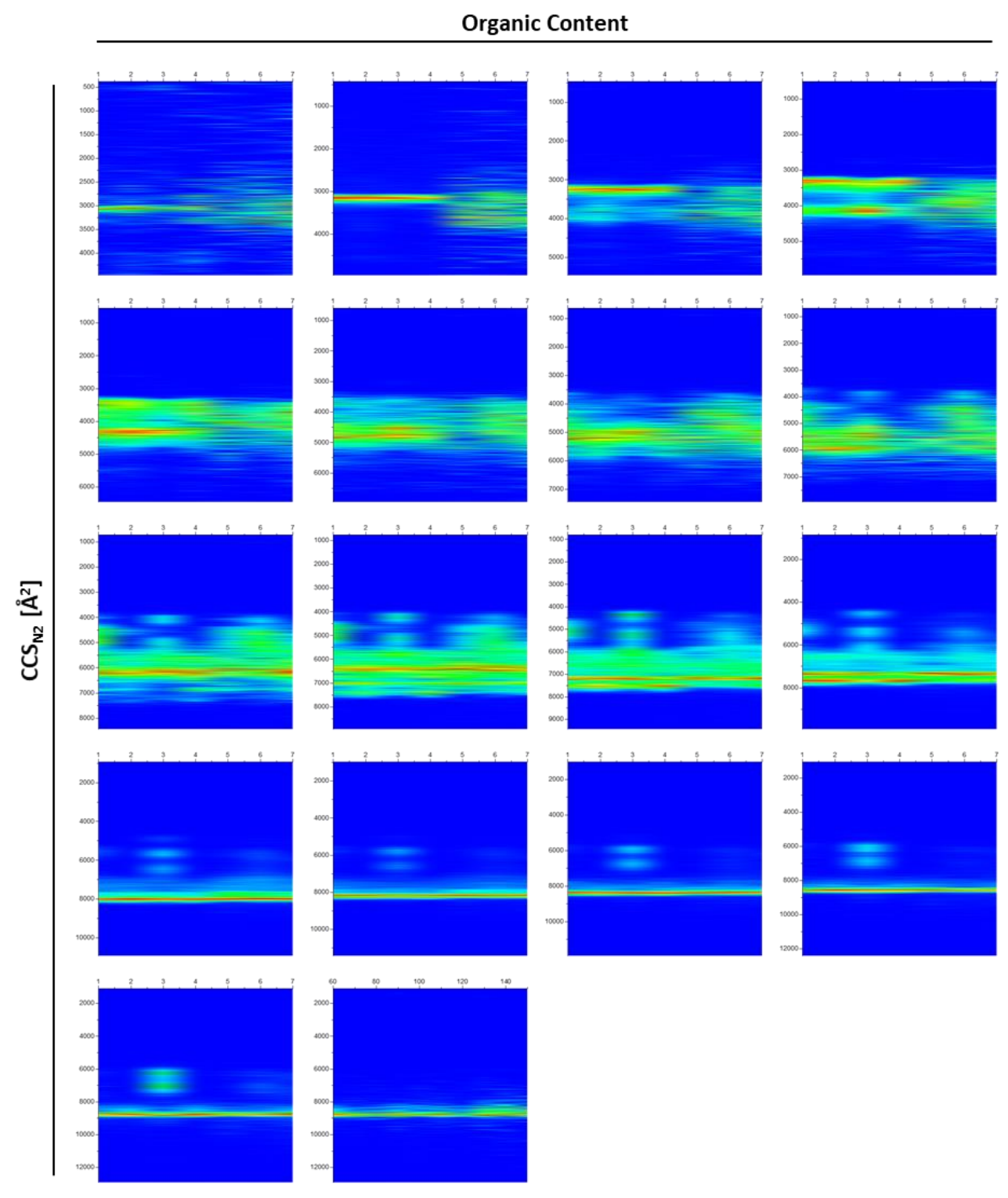


Figure S6.3. Contour plots of the charge states of $\mathrm{UCH}$ as a function of the activation energy (e.g., deflector voltage)

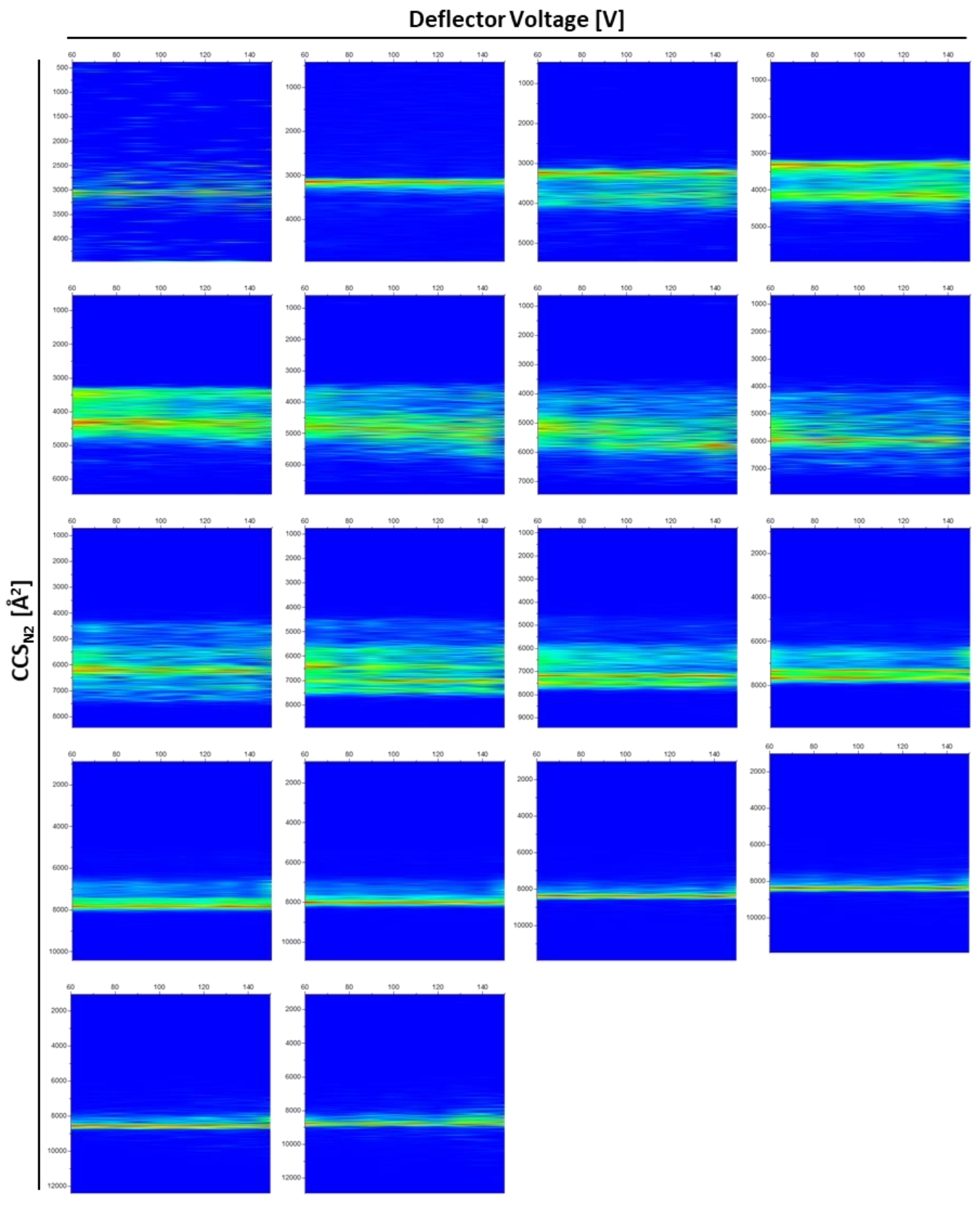


Table S6.1. Experimental and theoretical $\mathrm{CCS}_{\mathrm{N} 2}$ of the observed fragments after digestion with CPDY.

\begin{tabular}{|c|c|c|c|c|}
\hline Fragment & $\begin{array}{c}\text { Mass } \\
\text { (Da) }\end{array}$ & $\begin{array}{l}\text { Charge } \\
\text { State }\end{array}$ & $\begin{array}{l}\text { Experimental } \mathrm{CCS}_{\mathrm{N} 2} \\
\qquad\left(\mathrm{~A}^{2}\right) \\
\text { Most Intense Band }\end{array}$ & $\begin{array}{c}\text { Theoretical CCS } \mathrm{N}_{\mathrm{N} 2}\left(\mathrm{~A}^{2}\right) \\
\text { Model preserves integrity } \\
\text { of the core }\end{array}$ \\
\hline \multirow{6}{*}{$1-243$} & \multirow{6}{*}{26623} & 8 & 2650 & 2638 \\
\hline & & 9 & 2665 & 2656 \\
\hline & & 10 & 2683 & 2679 \\
\hline & & 14 & 4783 & 2758 \\
\hline & & 17 & 6113 & 2823 \\
\hline & & 18 & 6431 & 2844 \\
\hline \multirow{5}{*}{$1-242$} & \multirow{5}{*}{26460} & 8 & 2645 & 2631 \\
\hline & & 9 & 2657 & 2653 \\
\hline & & 11 & 2692 & 2701 \\
\hline & & 19 & 7121 & 2867 \\
\hline & & 21 & 7778 & 2934 \\
\hline $1-241$ & 26297 & 8 & 2613 & 2627 \\
\hline
\end{tabular}




\begin{tabular}{|c|c|c|c|c|}
\hline & & 9 & 2658 & 2644 \\
\hline & & 15 & 5176 & 2750 \\
\hline & & 16 & 5233 & 2773 \\
\hline & & 17 & 5241 & 2802 \\
\hline & & 14 & 4721 & 2734 \\
\hline & & 15 & 4997 & 2751 \\
\hline & & 17 & 5980 & 2803 \\
\hline & & 18 & 6138 & 2818 \\
\hline & & 11 & 3054 & 2671 \\
\hline & & 13 & 3378 & 2711 \\
\hline & & 8 & 1923 & 2589 \\
\hline & & 14 & 4756 & 2688 \\
\hline $1-228$ & 24693 & 16 & 5321 & 2731 \\
\hline & & 13 & 3884 & 2667 \\
\hline & & 18 & 5690 & 2821 \\
\hline $1-224$ & 24226 & 14 & 3983 & 2773 \\
\hline
\end{tabular}




\begin{tabular}{|c|c|c|c|c|}
\hline $1-220$ & 23739 & 13 & 3856 & 2629 \\
\hline $1-218$ & 23512 & 15 & 4973 & 2659 \\
\hline $1-216$ & 23268 & 15 & 4902 & 2643 \\
\hline $1-212$ & 22814 & 14 & 4538 & 2617 \\
\hline $1-209$ & 22457 & 19 & 6740 & 2688 \\
\hline $1-207$ & 22301 & 12 & 2974 & 2560 \\
\hline $1-201$ & 21555 & 9 & 2036 & 2481 \\
\hline 1-197 & 21129 & 16 & 4893 & 2523 \\
\hline $1-192$ & 20571 & 14 & 4348 & 2551 \\
\hline $1-189$ & 20314 & 15 & 5023 & 2553 \\
\hline $1-186$ & 20030 & 15 & 4829 & 2530 \\
\hline $1-183$ & 19791 & 14 & 4728 & 2504 \\
\hline $1-179$ & 19391 & 13 & 1725 & 2451 \\
\hline $1-177$ & 19164 & 10 & 2856 & 2409 \\
\hline $1-170$ & 18369 & 10 & 1984 & 2349 \\
\hline $1-168$ & 18126 & 11 & 2957 & 2392 \\
\hline
\end{tabular}




\begin{tabular}{|c|c|c|c|c|}
\hline $1-164$ & 17567 & 9 & 2785 & 2232 \\
\hline $1-160$ & 17067 & 13 & 3358 & 2284 \\
\hline $1-155$ & 16511 & 12 & 3326 & 2230 \\
\hline $1-149$ & 15838 & 14 & 3685 & 2253 \\
\hline $1-145$ & 15303 & 13 & 3580 & 2210 \\
\hline $3-244$ & 26568 & 15 & 5103 & 2776 \\
\hline $8-244$ & 26053 & 9 & 2613 & 2632 \\
\hline $13-244$ & 25493 & 14 & 4658 & 2704 \\
\hline $15-244$ & 25306 & 13 & 4384 & 2633 \\
\hline $17-244$ & 25049 & 14 & 4584 & 2643 \\
\hline $20-244$ & 24663 & 11 & 2554 & 2568 \\
\hline $24-244$ & 24127 & 16 & 5428 & 2651 \\
\hline $29-244$ & 23643 & 17 & 4856 & 2659 \\
\hline $3-243$ & 26029 & 9 & 2650 & 2638 \\
\hline $3-238$ & 25591 & 15 & 5023 & 2697 \\
\hline $7-203$ & 20879 & 8 & 2485 & 2423 \\
\hline
\end{tabular}




\begin{tabular}{|c|c|c|c|c|}
\hline $17-188$ & 18064 & 10 & 2328 & 2296 \\
\hline $18-174$ & 16531 & 14 & 3294 & 2259 \\
\hline $38-126$ & 8940 & 6 & 2404 & 1823 \\
\hline $49-113$ & 6250 & 5 & 2365 & 1754 \\
\hline
\end{tabular}


SUPPORTING INFORMATION FOR CHAPTER 7. ELUCIDATION OF THE

STRUCTURAL INTEGRITY AND STABILITY OF THE KNOT PROTEIN HALO

ACID DEHALOGENASE USING TRAPPED ION MOBILITY SPECTROMETRY MASS SPECTROMETRY

Figure S7.1. TIMS instrument scheme

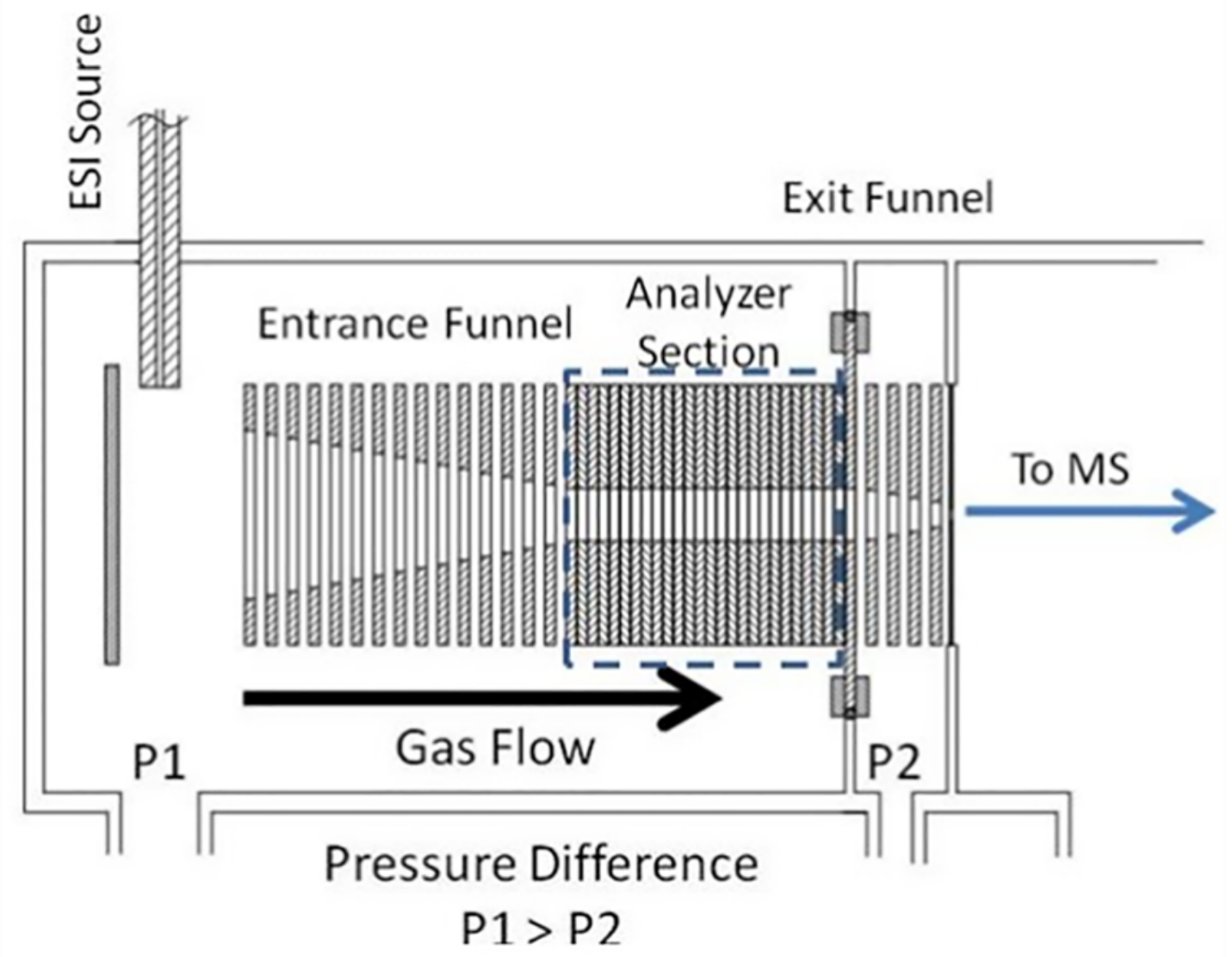


Figure S7.2. Contour plots of the charge states of DehI as a function of the organic content (e.g., \% MeOH). The solution conditions are labeled as follows: 1: $0 \% \mathrm{MeOH} ; 2: 5 \%$ $\mathrm{MeOH}$; 3: 10\% MeOH; 4: 20\% MeOH; 5: 30\% MeOH; 6: 40\% MeOH; 7: 50\% MeOH.

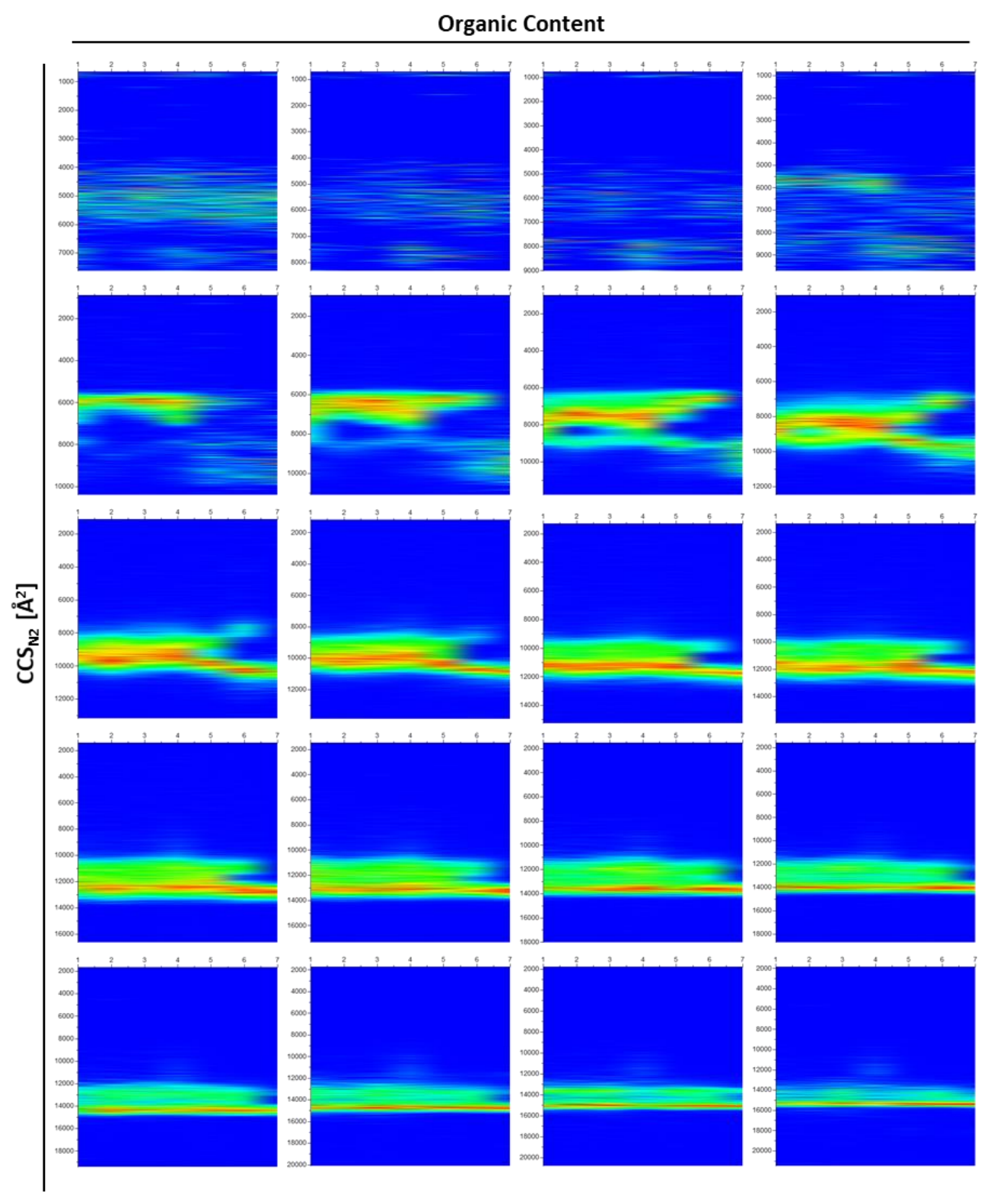


Figure S7.3. Contour plots of the charge states of DehI as a function of the activation energy (e.g., deflector voltage)

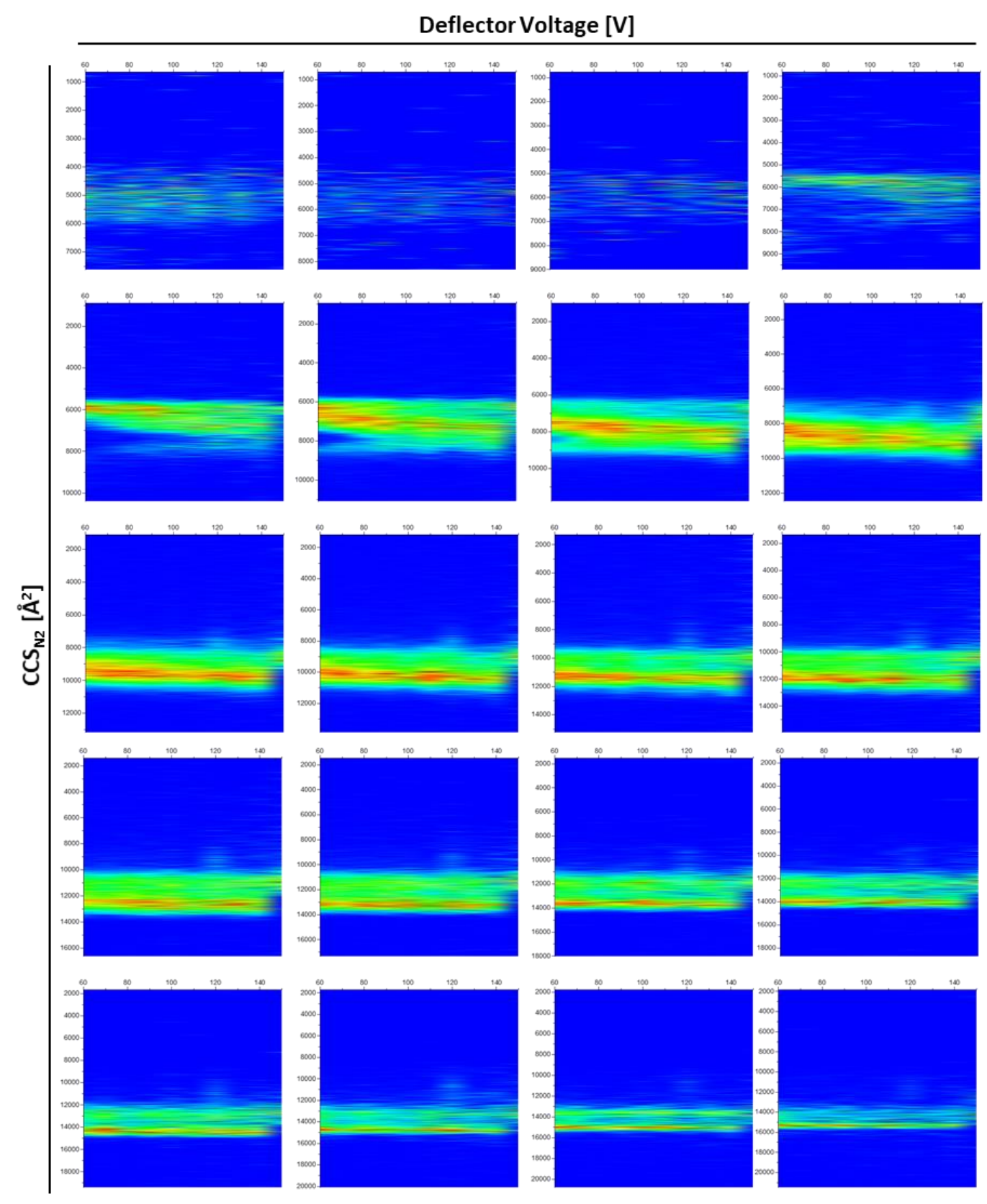


Table S7.1. Experimental and theoretical $\mathrm{CCS}_{\mathrm{N} 2}$ of the observed fragments after digestion with CPDY.

\begin{tabular}{|c|c|c|c|c|}
\hline Fragment & $\begin{array}{c}\text { Mass } \\
(\mathrm{Da})\end{array}$ & $\begin{array}{l}\text { Charge } \\
\text { State }\end{array}$ & $\begin{array}{l}\text { Experimental } \mathrm{CCS}_{\mathrm{N} 2} \\
\qquad\left(\mathrm{~A}^{2}\right) \\
\text { Most Intense Band }\end{array}$ & $\begin{array}{c}\text { Theoretical CCS } \mathrm{N}_{\mathrm{N} 2}\left(\mathrm{~A}^{2}\right) \\
\text { Model preserves integrity } \\
\text { of the core }\end{array}$ \\
\hline \multirow{6}{*}{$1-325$} & \multirow{6}{*}{36247} & 8 & 3177 & 3229 \\
\hline & & 12 & 4803 & 3301 \\
\hline & & 13 & 5178 & 3326 \\
\hline & & 14 & 5633 & 3338 \\
\hline & & 18 & 8687 & 3413 \\
\hline & & 19 & 9300 & 3422 \\
\hline \multirow{6}{*}{$1-323$} & \multirow{6}{*}{35920} & 8 & 3170 & 3212 \\
\hline & & 9 & 3568 & 3234 \\
\hline & & 11 & 4353 & 3279 \\
\hline & & 13 & 5111 & 3303 \\
\hline & & 14 & 5621 & 3327 \\
\hline & & 15 & 5880 & 3344 \\
\hline
\end{tabular}




\begin{tabular}{|c|c|c|c|c|}
\hline & & 17 & 7614 & 3380 \\
\hline \multirow{6}{*}{$1-320$} & \multirow{6}{*}{35509} & 18 & 8612 & 3663 \\
\hline & & 21 & 10538 & 3720 \\
\hline & & 25 & 12748 & 3802 \\
\hline & & 26 & 13498 & 3817 \\
\hline & & 28 & 14264 & 3844 \\
\hline & & 29 & 14629 & 3851 \\
\hline \multirow{5}{*}{$1-318$} & \multirow{5}{*}{35234} & 9 & 3220 & 3181 \\
\hline & & 10 & 3684 & 3210 \\
\hline & & 13 & 5074 & 3271 \\
\hline & & 14 & 5539 & 3282 \\
\hline & & 15 & 5801 & 3306 \\
\hline \multirow{2}{*}{$1-315$} & \multirow{2}{*}{34882} & 14 & 5401 & 3382 \\
\hline & & 17 & 7492 & 3436 \\
\hline \multirow{2}{*}{$1-309$} & \multirow{2}{*}{34173} & 8 & 2984 & 2977 \\
\hline & & 9 & 3399 & 2999 \\
\hline
\end{tabular}




\begin{tabular}{|c|c|c|c|c|}
\hline \multirow{2}{*}{$1-306$} & \multirow{2}{*}{33762} & 8 & 2955 & 2946 \\
\hline & & 14 & 5428 & 3026 \\
\hline \multirow{2}{*}{$1-298$} & \multirow{2}{*}{33008} & 19 & 9102 & 3219 \\
\hline & & 21 & 10447 & 3287 \\
\hline \multirow{3}{*}{$1-283$} & \multirow{3}{*}{31292} & 10 & 3768 & 2769 \\
\hline & & 13 & 4993 & 2816 \\
\hline & & 15 & 5701 & 3855 \\
\hline $1-266$ & 29352 & 8 & 2784 & 2588 \\
\hline $1-243$ & 26750 & 14 & 5406 & 2760 \\
\hline \multirow{2}{*}{$1-238$} & \multirow{2}{*}{26150} & 9 & 3246 & 2451 \\
\hline & & 18 & 8359 & 2674 \\
\hline $1-226$ & 24847 & 8 & 2845 & 2338 \\
\hline \multirow{3}{*}{$1-221$} & \multirow{3}{*}{24163} & 14 & 5237 & 2556 \\
\hline & & 15 & 5482 & 2573 \\
\hline & & 16 & 6372 & 2589 \\
\hline $1-207$ & 22617 & 10 & 3462 & 2332 \\
\hline
\end{tabular}




\begin{tabular}{|c|c|c|c|c|}
\hline $1-196$ & 21465 & 10 & 3436 & 2325 \\
\hline $1-183$ & 19868 & 11 & 3952 & 2348 \\
\hline $1-170$ & 18412 & 14 & 5273 & 2423 \\
\hline $1-148$ & 16099 & 9 & 3027 & 1996 \\
\hline \multirow{5}{*}{$7-326$} & \multirow{5}{*}{35664} & 8 & 3176 & 3156 \\
\hline & & 9 & 3585 & 3167 \\
\hline & & 10 & 3963 & 3201 \\
\hline & & 11 & 4364 & 3217 \\
\hline & & 12 & 4788 & 3231 \\
\hline \multirow{2}{*}{$12-326$} & \multirow{2}{*}{35234} & 10 & 3926 & 3178 \\
\hline & & 13 & 5132 & 3227 \\
\hline \multirow{4}{*}{$16-326$} & \multirow{4}{*}{34846} & 8 & 3027 & 3012 \\
\hline & & 11 & 4026 & 3046 \\
\hline & & 18 & 8674 & 3184 \\
\hline & & 19 & 9133 & 3202 \\
\hline $20-326$ & 34365 & 8 & 3054 & 2967 \\
\hline
\end{tabular}




\begin{tabular}{|c|c|c|c|c|}
\hline & & 9 & 3488 & 2981 \\
\hline & & 10 & 3782 & 3000 \\
\hline \multirow{3}{*}{$23-326$} & \multirow{3}{*}{33939} & 17 & 7568 & 3403 \\
\hline & & 21 & 10573 & 3470 \\
\hline & & 22 & 11034 & 3484 \\
\hline \multirow{2}{*}{$27-326$} & \multirow{2}{*}{33610} & 8 & 2989 & 2867 \\
\hline & & 14 & 5501 & 2978 \\
\hline $28-326$ & 33538 & 8 & 2977 & 2855 \\
\hline \multirow{2}{*}{$31-326$} & \multirow{2}{*}{33122} & 12 & 4687 & 2889 \\
\hline & & 17 & 7462 & 2991 \\
\hline \multirow{2}{*}{$39-326$} & \multirow{2}{*}{32182} & 8 & 2920 & 2651 \\
\hline & & 9 & 3346 & 2667 \\
\hline $43-326$ & 31827 & 8 & 2864 & 2611 \\
\hline \multirow{2}{*}{$48-326$} & \multirow{2}{*}{31273} & 10 & 3725 & 2779 \\
\hline & & 12 & 4583 & 2811 \\
\hline $60-326$ & 29995 & 8 & 2745 & 2483 \\
\hline
\end{tabular}




\begin{tabular}{|c|c|c|c|c|}
\hline \multirow{2}{*}{$66-326$} & \multirow{2}{*}{29416} & 9 & 3172 & 2491 \\
\hline & & 15 & 5462 & 2573 \\
\hline $74-326$ & 28476 & 8 & 2701 & 2413 \\
\hline $83-326$ & 27369 & 16 & 6124 & 2791 \\
\hline $90-326$ & 26661 & 11 & 3784 & 2478 \\
\hline \multirow{2}{*}{$99-326$} & \multirow{2}{*}{25663} & 8 & 2647 & 2357 \\
\hline & & 13 & 4736 & 2446 \\
\hline \multirow{2}{*}{$113-326$} & \multirow{2}{*}{24180} & 8 & 2638 & 2316 \\
\hline & & 10 & 3528 & 2350 \\
\hline $123-326$ & 22922 & 8 & 2602 & 2268 \\
\hline $133-326$ & 21895 & 8 & 2561 & 2204 \\
\hline $148-326$ & 20309 & 8 & 2528 & 2178 \\
\hline $89-312$ & 25980 & 7 & 2355 & 2317 \\
\hline $90-313$ & 24933 & 14 & 4782 & 2641 \\
\hline $87-310$ & 24920 & 18 & 8036 & 2796 \\
\hline $83-308$ & 25072 & 8 & 2545 & 2265 \\
\hline
\end{tabular}




\begin{tabular}{|c|c|c|c|c|}
\hline $80-304$ & 25045 & 6 & 2289 & 2139 \\
\hline $79-300$ & 24793 & 7 & 2336 & 2130 \\
\hline $77-291$ & 24040 & 10 & 3467 & 2311 \\
\hline $71-283$ & 23749 & 13 & 4017 & 2374 \\
\hline $65-277$ & 23856 & 14 & 4073 & 2426 \\
\hline $64-263$ & 22338 & 10 & 3234 & 2201 \\
\hline
\end{tabular}




\section{VITA}

\section{JUAN CAMILO MOLANO-ARÉVALO}

Born, Medellín, Colombia

2005-2009

B.A., Biology

Universidad de Antioquia

Medellín, Colombia

$2013-2018$

Doctoral Candidate

Florida International University

Miami, Florida

Teaching Assistant

Florida International University

Miami, Florida

\section{PUBLICATIONS AND PRESENTATIONS}

J. C. Molano-Arevalo, W. Gonzalez, K. Jeanne Dit Fouque, J. Miksovska, P. Maitre, F. Fernandez-Lima. "Insights from ion mobility-mass spectrometry, infrared spectroscopy, and molecular dynamics on Nicotinamide Adenine Dinucleotide structural dynamics: $\mathrm{NAD}^{+}$vs. NADH”. Phys. Chem. Chem. Phys. 2018, 20, 7043-7052.

J. C. Molano-Arevalo, K. Jeanne Dit Fouque, K. Pham, J. Miksovska, M. E. Ridgeway, M. A. Park, F. Fernandez-Lima. "Characterization of Intramolecular Interactions of Cytochrome c Using Hydrogen Deuterium Exchange - Trapped Ion Mobility - Mass Spectrometry and Molecular Dynamics”. Anal. Chem., 2017.

P. Benigni, R. Marin, J. C. Molano-Arevalo, A. Garabedian, J. J. Wolff, M. E. Ridgeway, M. A. Park, F. Fernandez-Lima, "Towards the analysis of high molecular weight proteins and protein complexes using TIMS-MS", Int. J. Ion Mobil. Spectrom., 19, 95-104, 2016.

W. G. Gonzalez, V. Ramos, M. Diaz, A. Garabedian, J. C. Molano-Arevalo, F. FernandezLima, J. Miksovska, "Characterization of the photophysical, thermodynamic and structural properties of the Terbium(III)-DREAM complex". Biochemistry, 55(12), 1873-1886, 2016.

J. C. Molano-Arevalo, D. R. Hernandez, W. G. Gonzalez, J. Miksovska, M. E. Ridgeway, M.A. Park, F. Fernandez-Lima, "Flavin Adenine Dinucleotide structural motifs: from solution to gas-phase", Anal. Chem., 86(20):10223-30, 2014. 
J. C. Molano-Arevalo, W. Gonzales, K. J. Dit Fouque, J. Miksovska, P. Maitre, F. Fernandez-Lima, "Insights from TIMS-MS, IRMPD Spectroscopy, and Molecular Dynamics on Nicotinamide Adenine Dinucleotide Structural Dynamics: NAD+ vs NADH”, 93rd Florida Annual Meeting and Exposition, Tarpon Spring, FL, May 4-6, 2017 (graduate student poster presentation).

J. C. Molano-Arevalo, K. Pham, J. Miksovska, M. E. Ridgeway, M. A. Park, F. FernandezLima, "Characterization of Cytochrome $c$ intramolecular interactions using nanoESIHDX-TIMS-MS and molecular dynamics." 25th International Conference on Ion Mobility Spectrometry, July 24-28, 2016 in Boston, Massachusetts. (graduate student poster presentation).

J. C. Molano-Arevalo, K. Pham, J. Miksovska, M. E. Ridgeway, M. A. Park, F. FernandezLima, "Characterization of Cytochrome c intramolecular interactions using nanoESIHDX-TIMS-MS and molecular dynamics.” ASMS 2016, June 5 - 9, 2016, St. Antonio, TX. (graduate student poster presentation).

J. C. Molano-Arevalo, W. Gonzales, J. Miksovska, P. Maître, F. Fernandez-Lima, "Insights from TIMS-MS, IR spectroscopy and molecular dynamics on Nicotinamide Adenine Dinucleotide structural dynamics: NAD+ vs NADH." 92nd Florida Annual Meeting and Exposition, Tampa, Florida. May 5-7, 2016. (graduate student oral presentation).

J. C. Molano. "Characterization of intramolecular interactions of cytochrome c using nanoESI-HDX-TIMS-MS and molecular dynamics". Statewide Graduate Student Competition, University of Florida, Florida. April 22nd, 2016. (graduate student poster presentation).

J. C. Molano-Arevalo, K. Pham, J. Miksovska, M. E. Ridgeway, M. A. Park, F. FernandezLima. "Characterization of intramolecular interactions of globular proteins using nanoESIHDX-TIMS-MS and molecular dynamics". 18th Annual Biomedical and Comparative Immunology Symposium. March 3-4, 2016, Miami, Florida. (graduate student oral presentation).

J. C. Molano-Arévalo, D. R. Hernández, W. G. González, J. Miksovska, M. E. Ridgeway, M. A. Park, F. Fernandez-Lima. "Flavin Adenine Dinucleotide structural motifs: from solution to gas-phase" International Society for Ion Mobility Spectrometry 2014, Asheville (graduate student oral presentation).

J. C. Molano-Arevalo, D. R. Hernandez, W. Gonzales, J. Miksovska, M. E. Ridgeway, M. A. Park, F. Fernandez-Lima. "Conformational Dynamics of Flavin Adenine Dinucleotide". Florida Annual Meeting and Exposition, Tampa, Florida, May 9-10, 2014. (graduate student oral presentation). 